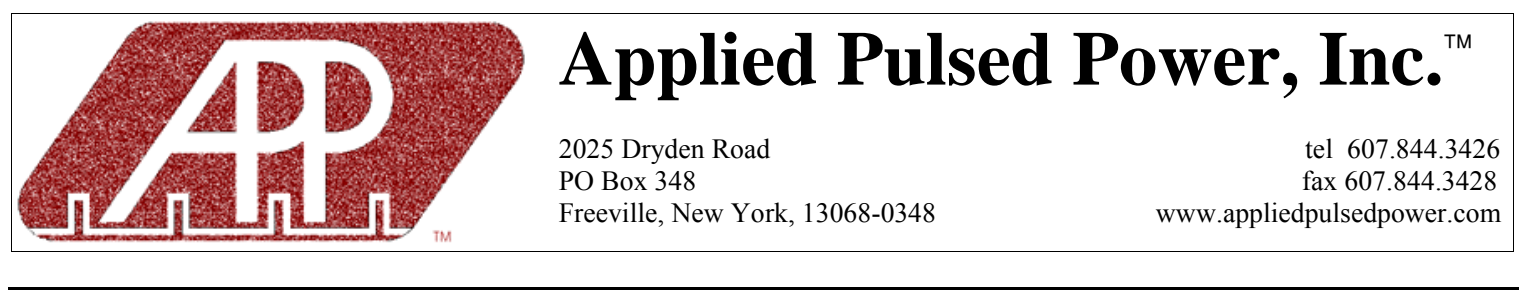

Pulsed, Inductively Generated, Streaming Plasma Ion Source for Heavy Ion Fusion Linacs

\author{
Steven C. Glidden, Howard D Sanders \\ Applied Pulsed Power, Inc. \\ John B. Greenly, Daniel L. Dongwoo \\ Laboratory for Plasma Studies \\ Cornell University
}

April 28, 2006

U.S. Department of Energy Phase II SBIR Final Report

Grant DE-FG02-01ER83147 


\section{Pulsed, Inductively Generated, Streaming Plasma Ion Source for Heavy Ion Fusion Linacs}

\section{Summary}

Heavy ion fusion accelerators require high current, high brightness ion sources. Ion sources presently in use that meet the stringent emittance requirements are capable of operation only at low current densities. A compact high current density ion source could substantially simplify and shrink the size and cost of the injector.

The goal of the work described for this program is the development of a compact, high current density, pulsed ion source, based on electrodeless, inductively driven gas breakdown to meet the requirements on normalized emittance, current density, uniformity and pulse duration for an ion injector in a heavy-ion fusion driver.

This project was carried out in two phases. Phase 1 used source configurations adapted from light ion sources to demonstrate the feasibility of the concept. In Phase 2 the performance of the source was enhanced and quantified in greater detail, a modulator for controlling the pulse shape was developed, and experiments were conducted with the ions accelerated to $>40 \mathrm{kV}$.

The plasma source produces $>10 \mu$ s pulse of Argon plasma with ion current densities $>100 \mathrm{~mA} / \mathrm{cm}^{2}$ at $30 \mathrm{~cm}$ from the source and with strongly axially directed ion energy of about $80 \mathrm{eV}$, and sub-eV transverse temperature. The source is capable of operating at the $<5 \%$ level in reproducibility and $10 \%$ in spatial uniformity over a $5 \mathrm{~cm}$ diameter. Control of the current density during the pulse has been demonstrated at the proof-of-principle level with the novel modulator coil method which allows attenuation of the ion current density without significantly affecting the beam quality. Simple acceleration experiments were performed as a means of producing a beam for diagnostic purposes. Within the limits of diagnostics that could be implemented with the simple, relatively low-voltage, single acceleration stage that was attainable in this program, the performance of the source was well characterized.

This program has developed the inductively-driven gas-breakdown streaming ion source to a level of performance ready to proceed to detailed engineering for implementation on a particular ion accelerator. The quality of the source is high enough that an accelerator is needed to further quantitatively diagnose the source in terms of ion energy spread and beam emittance. The coupling of the source of streaming ions to the initial extraction and acceleration gap of the accelerator can best be examined by fielding the source on an accelerator capable of accelerating, transporting and diagnosing an ion beam at the high current level the source provides.

The ion source is an important component of a heavy ion fusion accelerator. Ion sources such as the one described here also have applications to other fusion power approaches, including high flux neutral beams and plasmoids for injection into magnetically confined plasmas and field reversed ion rings. There are also significant commercial applications for the source, including, pulsed plasmas used as ion sources for surface treatment of materials, electric (plasma) thrusters for satellite station keeping and interplanetary spacecraft propulsion, and plasma sputter etch systems used extensively in the manufacture of semiconductors. Inductive generation of the plasma is of particular benefit to these applications. 


\title{
Pulsed, Inductively Generated, Streaming Plasma Ion Source for Heavy Ion Fusion Linacs
}

\author{
Phase II Final Report
}

\section{Contents}

$\begin{array}{ll}\text { 1.0 Introduction } & 5\end{array}$

$\begin{array}{lr}\text { 1.1 Applications } & 6\end{array}$

1.2 Program Objectives $\quad 7$

$\begin{array}{ll}\text { 1.2.1 Phase I } & 7\end{array}$

$\begin{array}{ll}\text { 1.2.2 Phase II } & 7\end{array}$

2.0 Summary of Phase I Program Results 9

$\begin{array}{ll}2.1 \text { Introduction } & 9\end{array}$

2.2 Streaming Plasma Ion Source Design 9

2.2.1 Radial Gas Flow Ion Source 11

2.2.2 Electrical Drivers 14

$\begin{array}{ll}\text { 2.2.3 Diagnostics } & 15\end{array}$

2.3 Radial Gas Flow Ion Source Performance 16

2.3.1 Directed Velocity 16

2.3.2 Divergence 16

2.3.3 Ion Current Density Dependence on Operating Parameters 19

$\begin{array}{ll}\text { 2.3.4 Reproducibility } & 20\end{array}$

2.3.5 Azimuthal Uniformity 21

2.3.6 Radial Distribution $\quad 24$

2.3.7 Effects of Driving Waveforms on Source Performance 27

2.3.8 Plenum Pressure $\quad 31$

2.3.9 Charge State Measurements $\quad 32$

2.4 Discussion of Phase I Results 32

$\begin{array}{ll}\text { 3.0 Phase II Source } & 34\end{array}$

3.1 Description of Phase II Source 34

3.1.1 Mechanical Structure of the Phase II Source 34

3.1.2 Electrical Structure of the Phase II Source $\quad 37$

3.1.3 ICCD Images of Phase II Source 38

3.1.4 Phase II Source Induced Magnetic Fields 47

3.1.5 Development of Operational Settings for the Phase II Source 51

3.2 Description of Modulator 53

3.3 Performance of Phase II Source $\quad 56$

3.3.1 Spectral Analysis of the Phase II Source $\quad 56$

3.3.2 Divergence Measurements of the Phase II Source $\quad 64$

3.3.3 Time of Flight Analysis of the Phase II Source 69 
$\begin{array}{ll}\text { 4.0 Accelerated Beam Experiments } & 72\end{array}$

4.1 Description of Ion Beam Acceleration System 72

$\begin{array}{ll}4.2 \text { Acceleration and Modulation } & 81\end{array}$

4.2.1 Acceleration and Modulation before the Changes in the Gap 81

4.2.2 Time of Flight Analysis of Accelerated and Modulated Ion Beam 86

4.2.3 Divergence data: Pepper Pot 90

$\begin{array}{ll}\text { 5.0 Project Summary } & 93\end{array}$

5.1 Discussion of source characteristics relative to HIF requirements 93

$\begin{array}{ll}5.2 \text { Accomplishments vs. program goals } & 94\end{array}$

$\begin{array}{ll}5.3 \text { Future directions } & 94\end{array}$

$\begin{array}{ll}\text { References } & 96\end{array}$

$\begin{array}{ll}\text { List of Figures } & 97\end{array}$

$\begin{array}{ll}\text { Appendices } & 100\end{array}$

$\begin{array}{lr}\text { A. Phase I Final Report } & 100\end{array}$

$\begin{array}{ll}\text { B. DPP03 Presentation } & 136\end{array}$

$\begin{array}{lr}\text { C. Patent Application } & 160\end{array}$ 


\subsection{Introduction}

Heavy ion fusion accelerators require high current, high brightness ion sources. Ion sources presently in use that meet the stringent emittance requirements are capable of operation only at low current densities. In order to obtain the desired beam current, many low current beams generated over a large area have to be focused down to a much smaller area over a distance of several meters, to inject into the main acceleration optics. This requires complex beam matching optics and adds greatly to the cost of the ion injector, becoming a significant part of the complexity, cost and technical risk of the accelerator. A compact high current density ion source could substantially simplify and shrink the size and cost of the injector [1].

The goal of the work described in this report is the development of a pulsed ion source, based on electrodeless, inductively driven gas breakdown [2] to meet the requirements on normalized emittance, current density, uniformity and pulse duration for the ion injector in a heavy-ion fusion driver. This source is based on our extensive previous experience with sources developed for intense pulsed ion diodes for US DOE and private industry, and for NASA as a high specific impulse plasma thruster for spacecraft propulsion.

The streaming plasma ion source uses a high speed gas valve to inject a puff of gas through a nozzle and into an annular volume between two driving coils. The coils are driven with a short period $(<3 \mu \mathrm{s}), 5-10 \mathrm{kV}$ ringing pulse. The current driven in the coils produces a rapidly rising magnetic field in the gas volume, inducing an azimuthal electric field in the gas of order $100 \mathrm{~V} / \mathrm{cm}$. This field initiates and drives a rapid breakdown in the gas and accelerates the resulting plasma. A more detailed description of the operation and performance of the source is presented in section 2 .

The inductively driven gas-breakdown plasma source produces a $>10 \mu$ s pulse of Argon plasma of $>100 \mathrm{~mA} / \mathrm{cm}^{2}$ ion current density, with strongly axially directed ion energy of about $80 \mathrm{eV}$, and sub-eV transverse temperature. The plasma is highly reproducible in its spatial, temporal and energy distributions. Several operating parameters, especially the driving voltage pulse shape, the distribution of gas provided by the pulsed valve to the source, and the geometry of the source and its driving coils, have been shown to strongly affect the plasma output. A modulator coil located downstream from the source is used to control the temporal shape of the steaming ion beam.

This project was carried out in two phases. Phase 1 used source configurations adapted from light ion sources to demonstrate the feasibility of the concept. The results of this effort are summarized in section 2.0. In Phase 2 the performance of the source was enhanced and quantified in greater detail, the modulator was developed, and experiments were conducted with the ions accelerated to $40 \mathrm{kV}$.

A comparison of the streaming plasma ion source and other sources that have been investigated for HIF is contained in the section discussing Phase I results. 


\subsection{Applications}

Fusion science and technology research is a valuable investment in the clean energy future of this country and of the world. The ion source is an important component of a heavy ion fusion accelerator. Ion sources such as the one described here also have applications to other fusion power approaches, including high flux neutral beams and plasmoids for injection into magnetically confined plasmas and field reversed ion rings.

Pulsed inductive plasma sources also have significant commercial applications. Applied Pulsed Power, Inc. (APP) is interested in developing devices and processes that depend upon the generation of high purity, partially or fully ionized plasmas from initially neutral gases. Three applications of particular interest to APP are: pulsed plasmas used as ion sources for surface treatment of materials, electric (plasma) thrusters for satellite station keeping and interplanetary spacecraft propulsion, and plasma sputter etch systems used extensively in the manufacture of semiconductors. Inductive generation of the plasma is of particular benefit to these applications.

An inductively generated, dense plasma source is a key feature of Applied Pulsed Power, Inc.'s MAP I ion diode system. The system is the heart of a unique, high throughput, industrial surface treatment system. QM Technologies in Albuquerque, New Mexico, mates APP's MAP I system with a $400 \mathrm{kV}$ pulser, producing a new thermal surface treatment which uses intense ion beams to enhance hardness, corrosion resistance and smoothness of surfaces, without chemicals or solvents. The ongoing development of this plasma source will help insure that the ion source developed for heavy ion fusion will meet repetition rate, reliability and lifetime requirements. The MAP I system primarily uses proton beams for the surface treatment. There are potential surface treatment applications which require higher $\mathrm{Z}$ ions. The results from the proposed Phase II program will aid in the development of plasma sources for such applications. A better understanding of the operation of the plasma source, and better design tools for future versions are essential to the success of this surface treatment technology.

The X-Line Thruster and certain NASA Advanced Propulsion Systems using field reversed configurations, will need well-controlled plasma sources with parameters suited to the pulsed inductive streaming source. There are commercial and government applications in station-keeping, orbital transfer, and other propulsion systems for satellites and spacecraft of a variety of types.

Plasma processing finds heavy use in semiconductors and other industries. The dense, high purity plasmas with highly directed ion velocity produced by APP's inductively ionized sources may find application there as well. Directed ion energy is of great value in controlling the shape of etched regions as chip device scales continue to shrink. 


\subsection{Program Objectives}

\subsubsection{Phase I}

In Phase I of the program, an experimental argon pulsed inductive plasma source was fabricated and used to conduct initial research with available drivers and plasma diagnostics. Modifications to the plasma source were made to obtain ion source parameters consistent with the requirements for standard accelerating structures as determined from a preliminary injector stage design. The goal of Phase I was to determine the likelihood that the pulsed inductive plasma source could produce the desired ion source characteristics for a heavy ion fusion induction linac.

Phase I Technical Objectives.

1. Using standard acceleration techniques, produce a preliminary design of the first stage of acceleration from which we can define the desired ion source parameters.

2. Design and fabricate an argon pulsed inductive plasma source based on sources developed previously for other applications.

3. Undertake an experimental program with detailed plasma diagnostics, and make modifications to the plasma source to obtain the source parameters indicated by the accelerator design.

4. Analyze the results from the source experiments and accelerator design to gauge the viability of this approach.

\subsubsection{Phase II}

The goals of the Phase II program were to enhance the performance of the source, improve our understanding of its operation, and to quantify the performance in sufficient detail to predict the characteristics of an accelerated beam using this source.

Phase II Technical Objectives

1. Develop an ionization coil driver with variable driving pulse shape and use this to determine the extent to which the driver can control the ion pulse shape.

2. Improve the gas nozzle design to increase the axial density gradient to facilitate operation at higher gas pressures to generate larger ion flux.

3. Optimize the azimuthal uniformity of the source by enhancing the uniformity of the high speed valve and decreasing the plenum size to allow operation near the peak of the gas pulse.

4. Optimize the radial uniformity by adjusting the magnetic field geometry of the ionization coils to best distribute the streaming ions.

5. The preceding objectives will be supported by detailed measurements of plasma conditions at the source as well as at the distance chosen for beam extraction. The local source measurements of density distribution in space and time will give 
improved understanding of the source operation, and make it possible to attain optimal performance.

6. Develop a beam extraction and acceleration stage to produce an $\sim 100 \mathrm{keV}$ extracted beam from this ion source, for the purpose of performing experiments to precisely quantify and improve the ion source performance. Experiments will be performed in 3 stages:

a. DC acceleration of a single beamlet

b. Pulsed acceleration of a single beamlet

c. Multiple beamlet acceleration.

7. Develop and employ accurate diagnostics for the extracted beam, with the precision necessary to evaluate performance with respect to heavy ion injector requirements. Quantities measured would include beam spatial and temporal uniformity, charge state distribution, and beam emittance. All measurements will test reproducibility and stability of the performance for multiple pulses. 


\subsection{Summary of Phase I Program Results}

\subsection{Introduction}

The Phase I project developed a basic configuration for an inductively driven gasbreakdown plasma source that produces a $>10 \mu$ sulse of Argon plasma of $>100 \mathrm{~mA} / \mathrm{cm}^{2}$ ion current density, with strongly axially directed ion energy of about $80 \mathrm{eV}$, and sub-eV transverse temperature. The plasma is highly reproducible in its spatial, temporal and energy distributions. Several operating parameters, especially the driving voltage pulse shape, the distribution of gas provided by the pulsed valve to the source, and the geometry of the source and its driving coils, have been shown to strongly affect the plasma output and offer promise of capability to adjust the output to offer high spatial and temporal uniformity. The Phase I project used basic plasma diagnostics to characterize the streaming plasma output of the source, and we describe the results in this section.

\subsection{Streaming Plasma Ion Source Design}

The Phase I experimental plasma ion source includes a high speed gas valve and its driver, a nozzle, ionization coils and their driver and related support hardware. Figure 2.2.1 is a block diagram of this source.

The high speed gas valve injects a puff of gas through a nozzle and into an annular volume between 2 driving coils. The coils are driven with a short period $(<3 \mu \mathrm{s}), 5-10$ $\mathrm{kV}$ ringing pulse. The current driven in the coils produces a rapidly rising magnetic field in the gas volume, inducing an azimuthal electric field in the gas of order $100 \mathrm{~V} / \mathrm{cm}$. This field initiates and drives a rapid breakdown in the gas and accelerates the resulting plasma.

The high speed gas valve is a standard APP product designed to generate annular gas puffs for the anode plasma source of an ion diode, and can operate at $>5$ pulses per second. The valve has a conical diaphragm that seals against an 'o' ring. A pulsed magnetic field is used to rapidly drive the diaphragm away from the 'o' ring, allowing gas from an annular plenum to flow radially outward. This results in a short $(<1 \mathrm{~ms})$ pulse of gas with a very fast $(<100 \mu \mathrm{s})$ rising leading edge. The valve used in Phase I has a plenum volume of 0.41 Torr liter at a plenum pressure of $3 \mathrm{~atm}, 0.23$ Torr liter at 1.6 atm and 0.084 Torr liter at $0.6 \mathrm{~atm}$. The timing between the high speed valve and ionization coil drivers is used to control the neutral gas density near the coils. 


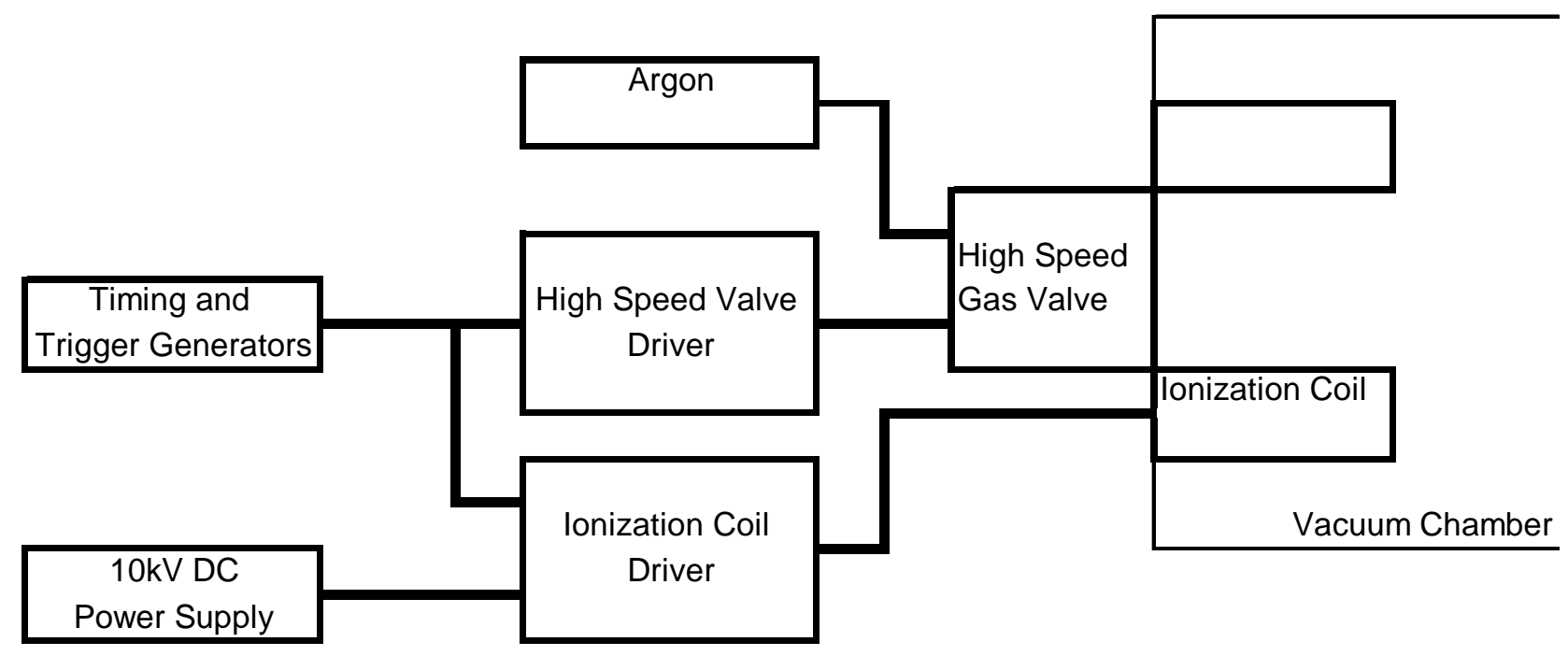

Figure 2.2.1. Block Diagram of the Plasma Source.

A characteristic of inductive ionization is the fact that without electrode phenomena that provide seed electrons (field enhancement and field emission, secondaries, photoelectrons) to begin the discharge, much higher electric fields may be required to initiate the ionization process. Since the ionization coil must have current driven in it to produce the changing magnetic flux that generates the inductive electric field, the higher that electric field required (or the longer it must be applied) to initiate the ionizing discharge, the more energy must be expended in the electric driving circuit. Furthermore, unless a complex driving circuit is employed, an initial high voltage on the ionization coil leads to high peak current in the ringing LC waveform, possibly far exceeding the current needed in the discharge to drive the plasma to full ionization.

We have developed a simple method[2] for providing an initiation mechanism that greatly reduces the driving voltage required. We add a single turn loop with a small gap located within the gas. This "preionizer" loop is inductively coupled to the ionizing coil, thus requiring no extra driving circuitry or energy input. By inserting a small $(<1 \mathrm{nF})$ capacitor in series with the loop, the loop current is limited to $<1 \mathrm{~A}$, so that plasma contamination and electrode erosion are essentially eliminated. The charge transferred through this gap is so small that its lifetime can be much greater than $10^{8}$ pulses. The very low-energy discharge across the gap triggers a distributed, volume discharge in the gas without significant current driven through the electrodes, while simultaneously eliminating the need for a higher electric field for pure inductive breakdown. The electrodes are used for what they do best - initiation. The inductive coil is used for what it does best; driving current without contamination or component wear.

The nozzle and ionization coil and preionizer winding forms are fabricated from plastic and the coils are epoxied in place. This fabrication method is reliable, quick and inexpensive, and allows substantial modifications and the fabrication of new coils during the course of the experiments. 
Several different nozzle and coil geometries were explored in Phase I. We will describe only the most successful approach here. A description of other configurations and a comparison of their performances is included in the Phase 1 final report, and appears as appendix A of this report.

\subsubsection{Radial Gas Flow Ion Source}

The radial gas flow source uses a conical nozzle to generate an annular ring of gas that has a very steep gradient in the axial direction. The purpose of this configuration is to minimize the amount of neutral gas downstream from the ionization region. Two versions of this configuration were explored. The version which produced the greatest ion current downstream of the source has the ionization coils located upstream of the nozzle and is shown in Figure 2.2.2. All ions are accelerated downstream. A magnetic field plot is shown in Figure 2.2.3. The magnetic field null is in the plastic coil form near the surface so that breakdown may be initiated at this surface. It is possible to remove material in this region if necessary to eliminate surface contamination, but this was not done in Phase I.

The version of the source shown in Figure 2.2 is similar to the ion source used to generate high current $(>10 \mathrm{kA})$ hydrogen and nitrogen ion beams for materials modification ${ }^{4}$.

Each coil has a single turn and both turns are connected in series. Because the aluminum valve body acts as a flux excluder, the coils are at a radius of $\sim 4 \mathrm{~cm}$. If it is desirable to shrink the size of the source, smaller valves could be built and have been built in the past. The third coil is used for preionization as described previously. 
Applied Pulsed Power, Inc.

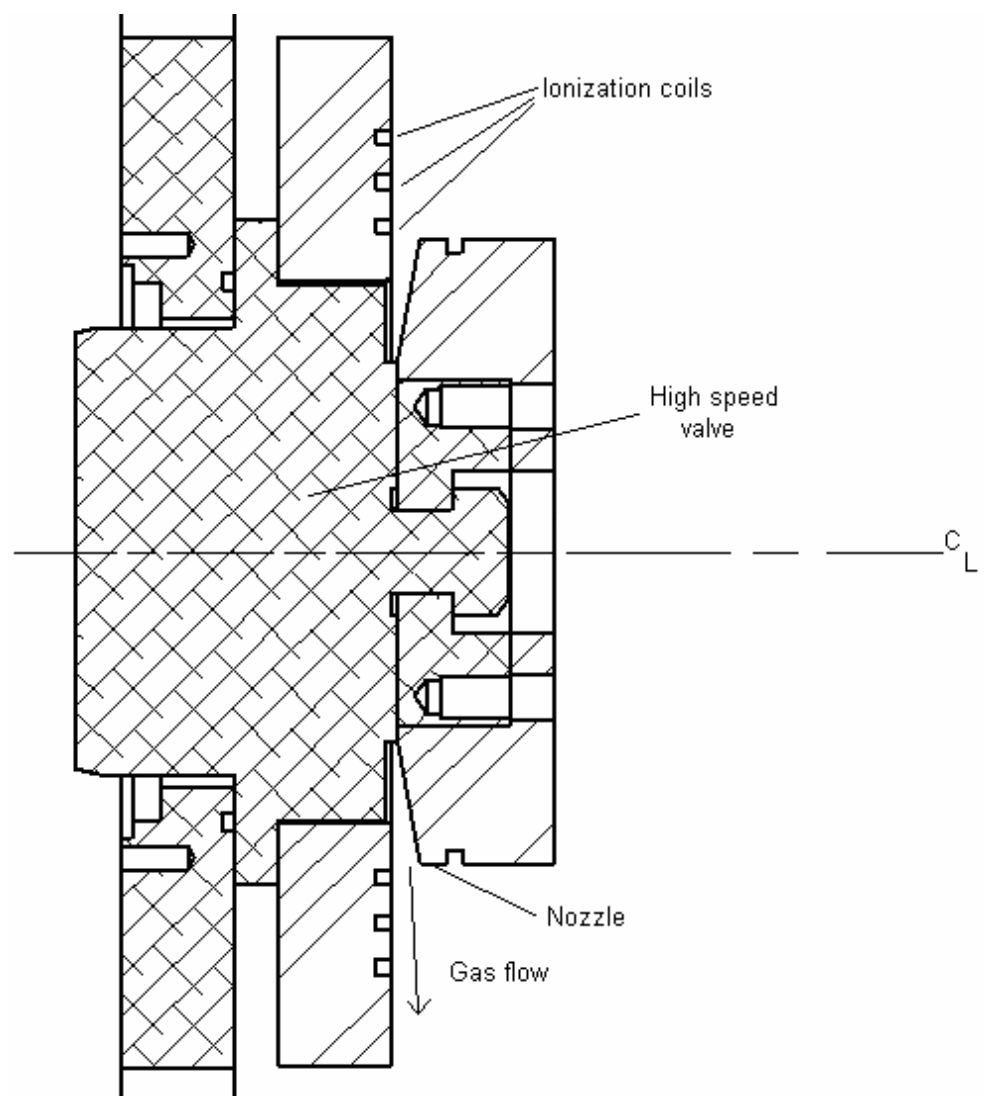

Figure 2.2.2. Section View of the Radial Gas Flow Source

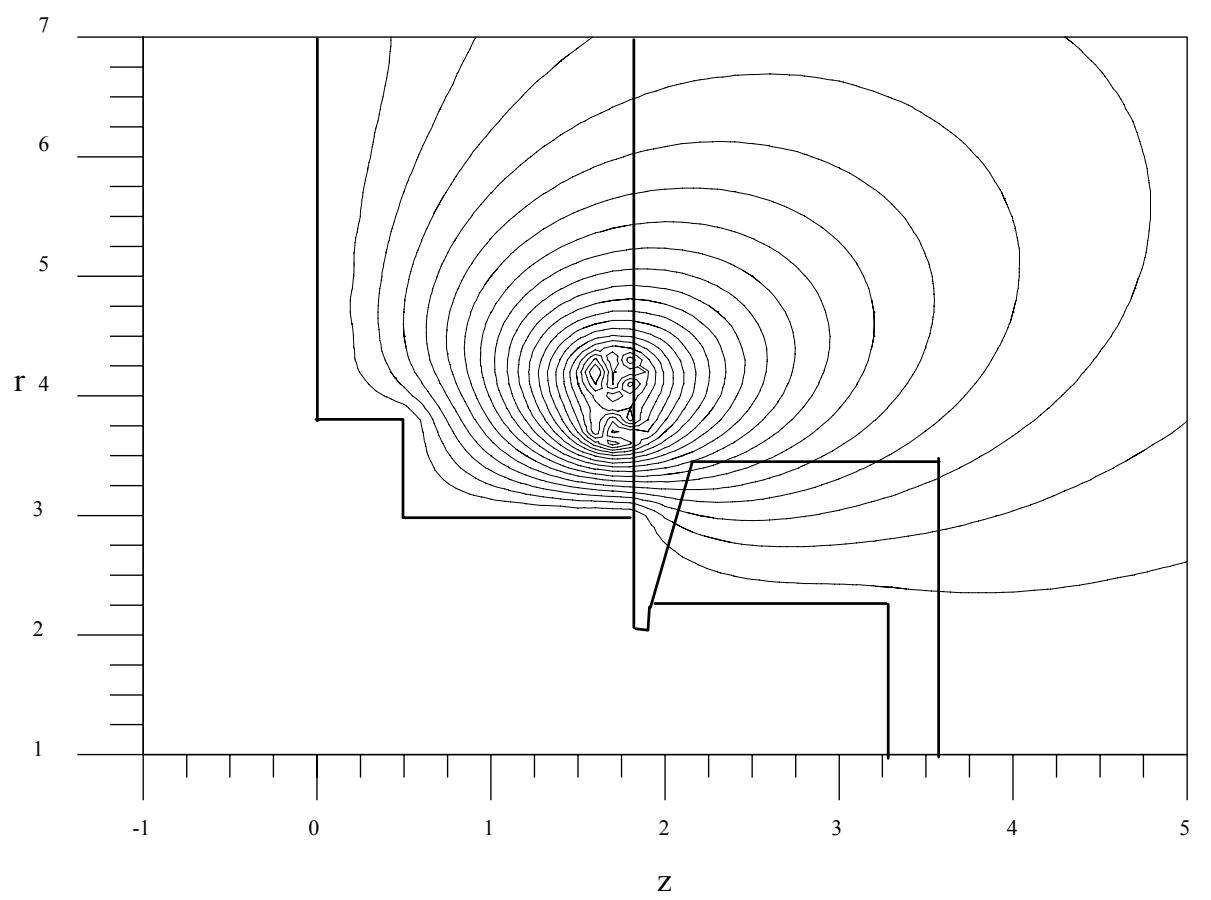

(b)

Figure 2.2.3. Magnetic Field Plot for the Radial Gas Flow Source. 
The radial flow source has a very steep axial gradient as a result of the design of the gas nozzle, which gives supersonic flow in this regime. A large axial gas density gradient in front of the coils is desirable because the ion flux from the source rapidly decreases as a result of collisions between the ions and the gas molecules, when the density of the neutral gas in front of the source exceeds $\sim 10$ mTorr. This behavior can be seen in Figure 2.2.4, which shows the peak ion flux measured $16 \mathrm{~cm}$ from the source at different valve to ionization coil driver delay times for the axial flow source. For delays longer than $210 \mu \mathrm{s}$, the ion flux quickly falls to zero, even though plasma is generated for delays $>300 \mu$ s as observed by the photomultiplier.

Another advantage of the radial gas flow is that in an accelerator, the ion extraction and acceleration electrodes could be much closer to the source without contamination from neutral gas, because most of the supersonic gas flow exits radially near the source and could be optimally pumped there.

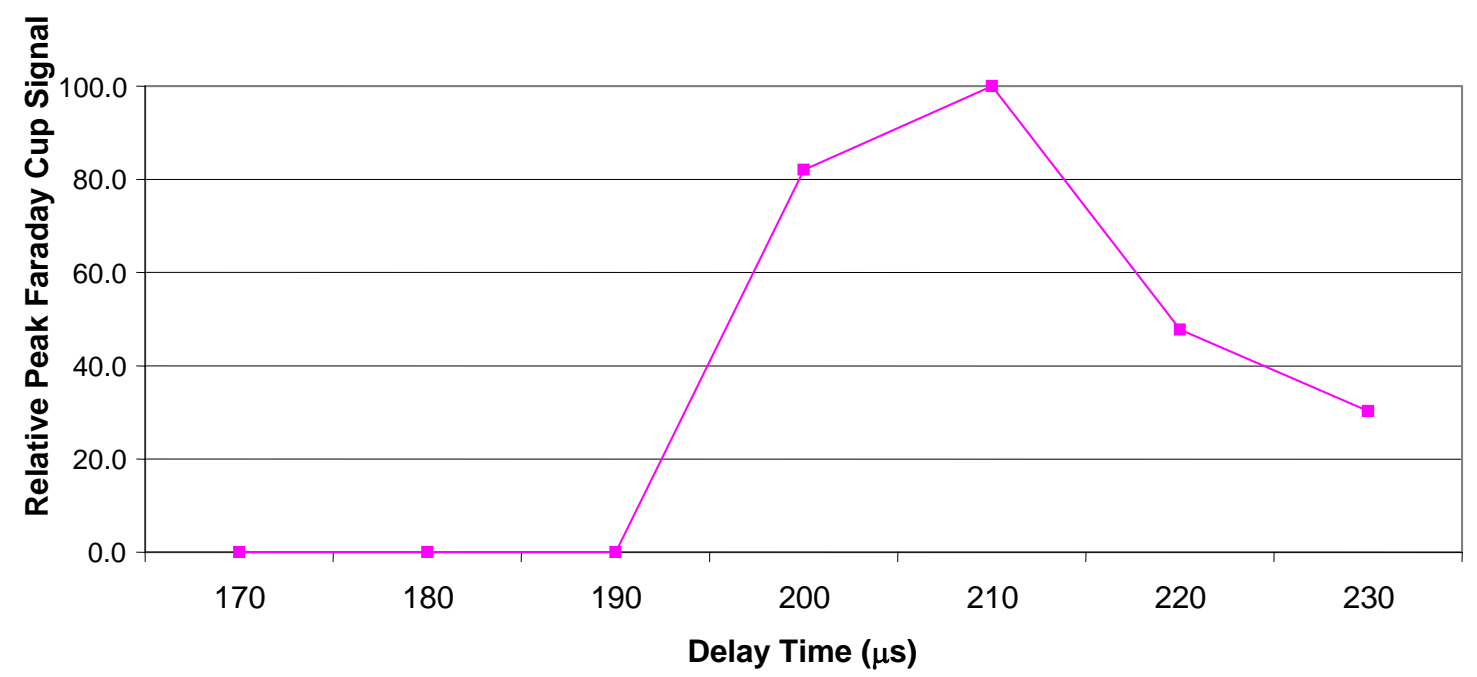

Figure 2.2.4. Relative Peak Ion Flux as a Function of the Time Delay Between the Valve and Ionization Coil Drivers.

The gas pressure near the ionization coils was measured using a Fast Ionization Gauge (FIG) (Figure 2.2.5). The FIG samples a region of gas of $3 \times 3 \times 10 \mathrm{~mm}$ without obstructing the flow of gas in the desired direction. The FIG response was nonlinear with argon above $\sim 50 \mathrm{mTorr}$, so the peak pressures indicated in the figure are approximate. 


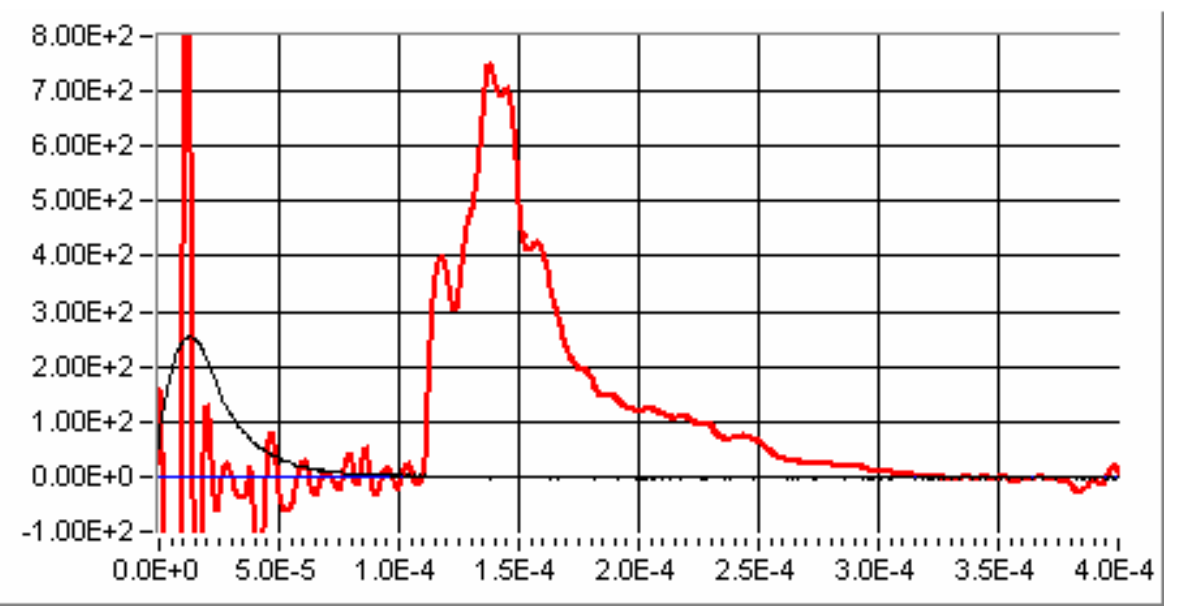

Figure 2.2.5. Fast Ionization Gauge Measurement of the Gas Pressure. Gas pressure is (mTorr) measured at a radius of $4 \mathrm{~cm}, 0.5 \mathrm{~cm}$ from the radial gas flow source. The first $100 \mu \mathrm{s}$ of the waveforms are noise pickup from the valve driver current (light trace).

\subsubsection{Electrical Drivers}

The ionization coil driver uses a solid state switch to discharge a capacitor charged to $<10 \mathrm{kV}$ through the ionization coils. The output capacitance, and thus the ringing frequency of the ionization coil current, is varied by adding additional capacitors. The driver has resistive voltage monitors and Rogowski coils for measuring output current. An additional switch was added to enable the ringing discharge to be truncated after a few cycles. Typical ionization coil driver current and voltage waveforms are shown in Figure 2.2.6. Also shown are the signal from a photomultiplier tube (PMT) that monitors the visible light output of the plasma.

The high speed valve driver is a standard APP product and generates a few kA, $30 \mu \mathrm{s}$ pulse which opens the valve.

The timing between the high speed valve and ionization coil drivers is independently adjusted. 


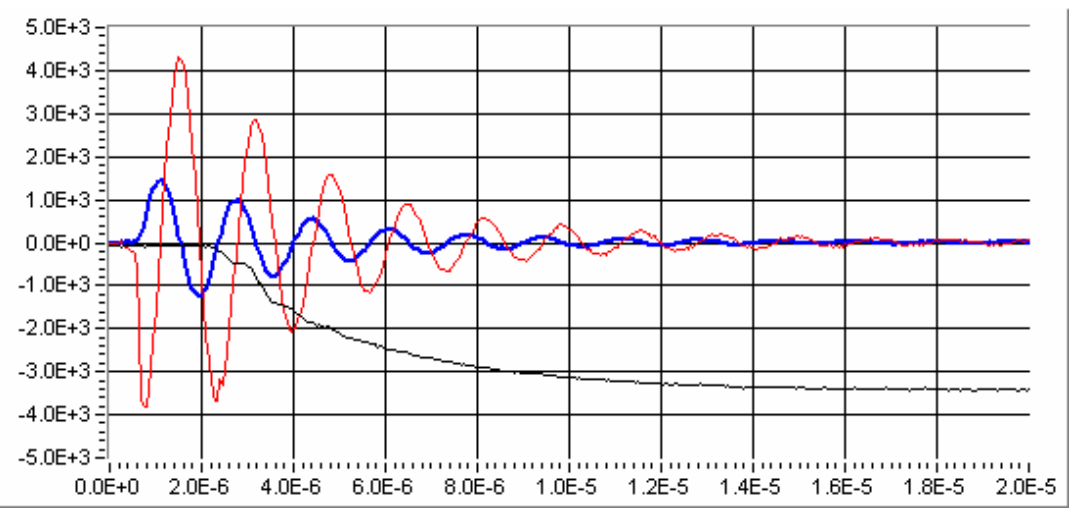

Ionization Coil Driver Output Voltage, V, Current (bold) A, and Photomultiplier Signal

Figure 2.2.6 Representative Ionization Coil Driver Waveforms, and PMT Signal from the Visible Light Output of the Plasma.

\subsubsection{Diagnostics}

Collimated aperture Faraday cups were the primary diagnostic used in exploring the behavior of the source. The Faraday cups had $1 \mathrm{~mm}$ diameter apertures, with a second positioned several $\mathrm{cm}$ in front. This configuration is shown in Figure 2.2.7. Unless otherwise noted, this configuration was used for the ion flux measurements presented here.

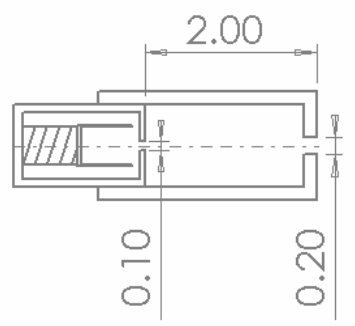

Figure 2.2.7. Collimated Aperture Faraday Cup; Dimensions in $\mathrm{cm}$.

A photomultiplier tube measured the onset of visible light from the breakdown of the argon gas. 


\subsection{Radial Gas Flow Ion Source Performance}

\subsubsection{Directed Velocity}

The ion velocity of $\sim 2 \mathrm{~cm} / \mu \mathrm{s}$ is inferred from signals from Faraday cup arrays separated by $8 \mathrm{~cm}$. By comparison, the thermal velocity of the argon gas is $\sim .04 \mathrm{~cm} / \mu$ s at $20 \mathrm{C}$.

\subsubsection{Divergence}

An array of Faraday cups with collimated apertures as shown in Figure 2.2.7 was used to examine beam divergence. The axis of collimation could be tilted at various angles relative to the axis of the source. The good reproducibility of the source enabled us to compare Faraday cup signals taken at 2 degree intervals from -10 to +10 degrees.

The resulting signals were analyzed in the following way to find the angular divergence of the ions in the streaming plasma. A Gaussian distribution was assumed for the intensity of the ion current density versus angle, corresponding to a thermal (Maxwellian) transverse ion velocity distribution. Taking into account the finite acceptance angle of the collimation, a Mathcad worksheet generating a curve for each angle of tilt was written. The curve plots the signal that would be obtained at that angle (normalized to the signal at zero angle) as a function of the Gaussian width shown as standard deviation (Figure 2.3.1a). The experimental data point for a given angle is then located on the curve to find the corresponding angular Gaussian standard deviation. The results are shown in Figure 2.3.1b. There is a pair of points at each angle; each resulting from a pair of measurements taken at that angle on opposite sides of zero degrees.

The results show that all angles from 4-10 degrees were consistent with a width of 5.7 +/0.5 degrees (Figure 2.3.1b). At 2 degrees, the finite collimator acceptance angle results in a very insensitive measurement, producing uncertainty too large for a useful fit. Excluding 2 degrees, the data conform to the 5.7 degree Gaussian standard deviation well within the measurement precision of $\sim 1$ degree.

Taking the axial velocity of $2 \mathrm{~cm} / \mu$ s gives $\sim 80 \mathrm{eV}$ axial energy for $\mathrm{Ar}+$ ions, and this standard deviation of 0.1 radian therefore corresponds to a transverse velocity one tenth of the axial velocity, or $\sim 0.8 \mathrm{eV}$ transverse ion energy. Similar sets of measurements under other operating conditions and distances from the source gave results from $0.5 \mathrm{eV}$ to $0.8 \mathrm{eV}$. Use of the usual Maxwellian thermal velocity definition thus gives $0.4-0.6 \mathrm{eV}$ ion "temperature".

The meaning of this measurement requires further elaboration. The axially streaming plasma passes through the first aperture of the collimator and then expands as it travels to the second aperture. This expansion is caused by two factors. The first is the transverse energy spread of the ions in the stream that enters the aperture, and if this were the only effect, the $0.6 \mathrm{eV}$ number would be a correct characterization. The second effect is that even in the absence of any transverse spread in the entering ion distribution, the plasma 
stream that is selected by the first aperture would be expected to expand transversely due to electron thermal pressure at the ion sound speed, which goes as $\left(\mathrm{Te} / \mathrm{m}_{\mathrm{i}}\right)^{1 / 2}$.

Thus, from this point of view, the expansion is a measure of Te. If the ions are much hotter than the electrons, the first effect dominates, but if the Te Ti, the electron effect dominates. If $\mathrm{Te}=\mathrm{Ti}$, this speed is faster than the ion thermal speed by the square root of the adiabatic index gamma, which for a single-species ion-electron plasma in this free transverse expansion, should be a factor of $\sim 1.3$. Thus the inferred $0.6 \mathrm{eV}$ is an upper limit for the transverse ion temperature. The ions could be colder, and the expansion in the collimator dominated by the electron temperature, but is probably unlikely in this plasma.

In any case, the low temperature is the consequence of the relatively "gentle" inductive driving of the gas ionization, essentially a pulsed, relatively low-frequency RF discharge, and possibly also of some cooling as the plasma flows away from the source. More detailed measurements in Phase II will be made in the source region to fully characterize the gas breakdown regime in this source, and identify the conditions which minimize the ion temperature. 


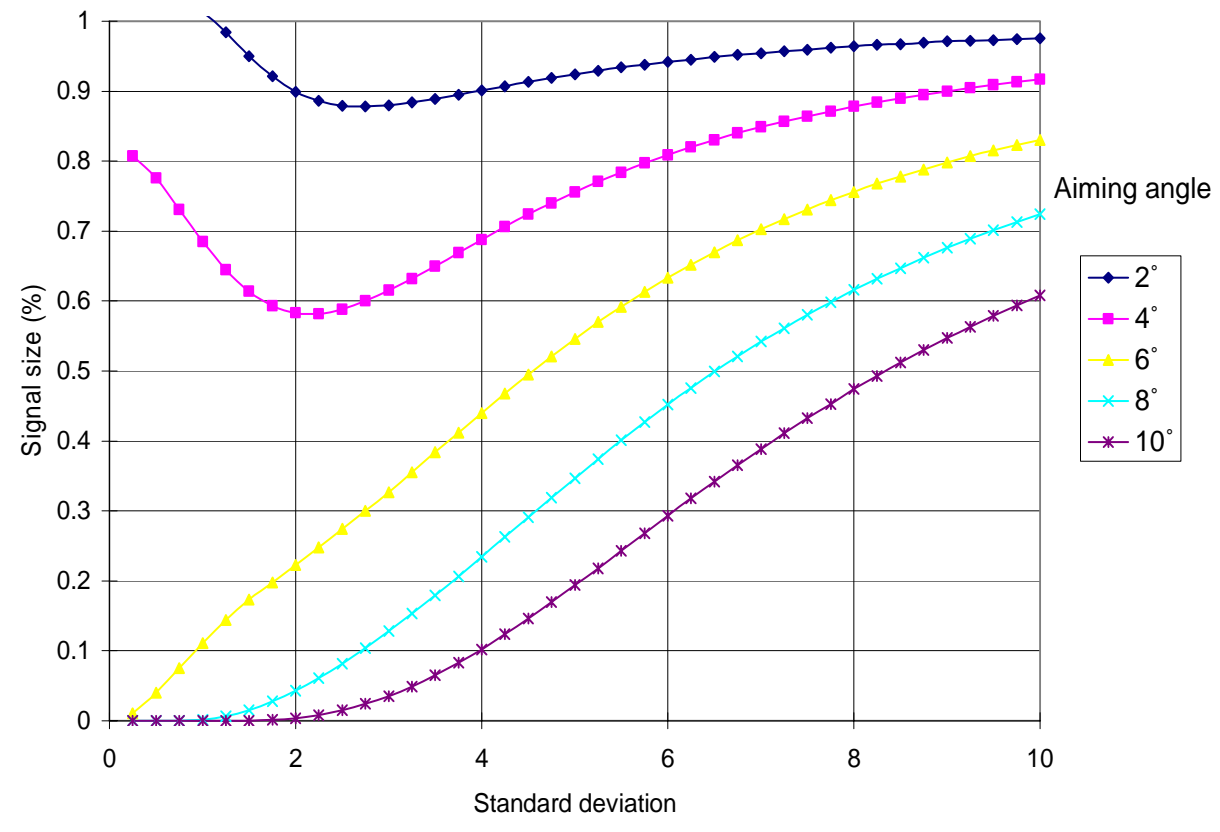

(a) Mathcad generated plots of the signal that would be obtained at different angles of tilt (normalized to the signal at zero angle) as a function of the Gaussian width.

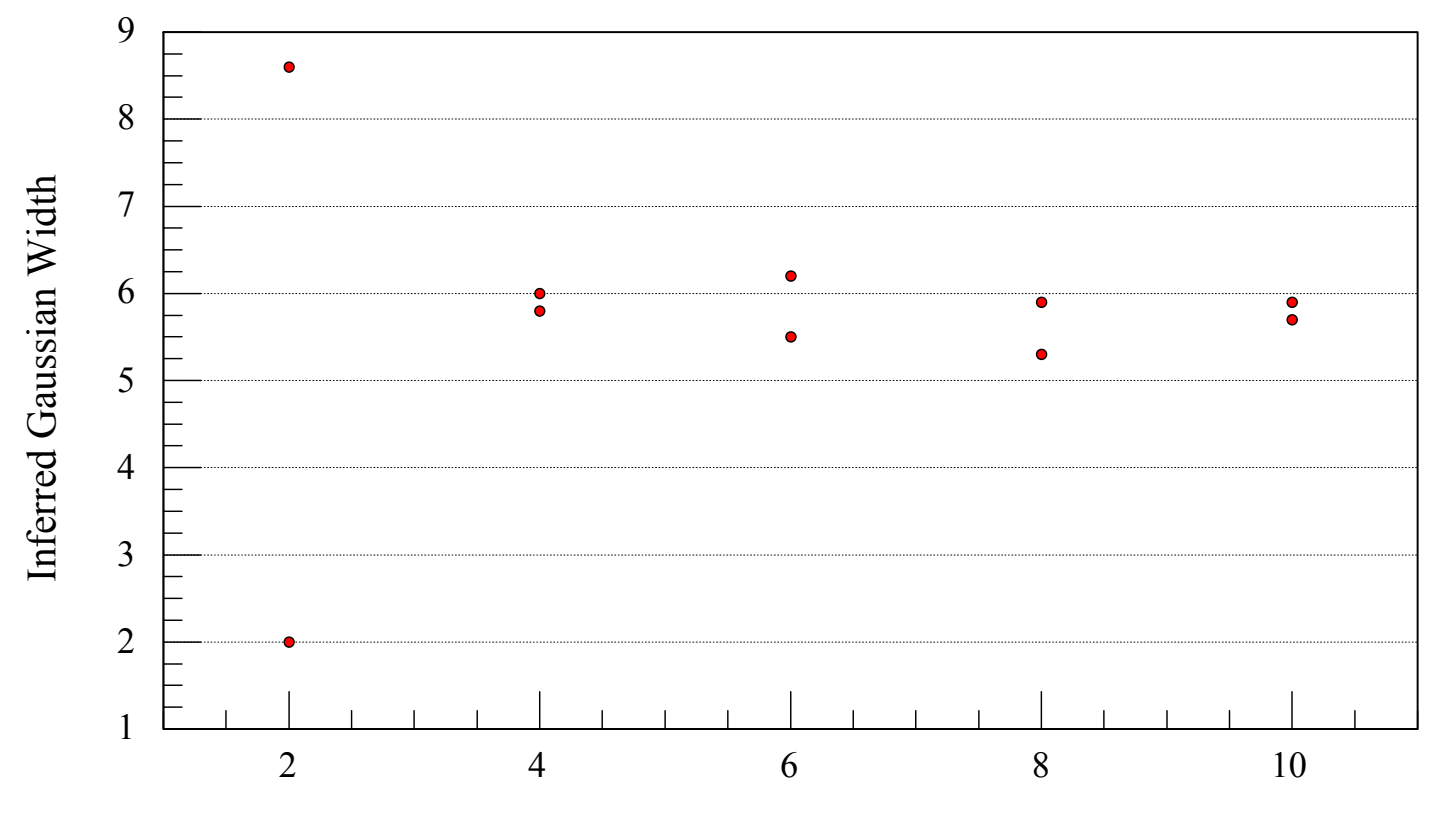

Angle of Measurement

(b) Inferred Gaussian standard deviation at different angles of tilt comparing collimated Faraday cup measurements with the plots in (a).

Figure 2.3.1 Angular Divergence Measurements. 


\subsubsection{Ion Current Density Dependence on Operating Parameters}

The design of the gas flow nozzle affects the output of the source. This is seen in Figure 2.3.2, which compares for the radial flow source, the output at $16 \mathrm{~cm}$ for 2 simple nozzle designs at different valve to coil delay times. The 10 degree nozzle produces a thinner layer of gas in front of the coils, achieving maximum ion flux at a longer delay time when there is a higher density in front of the ionization coils.
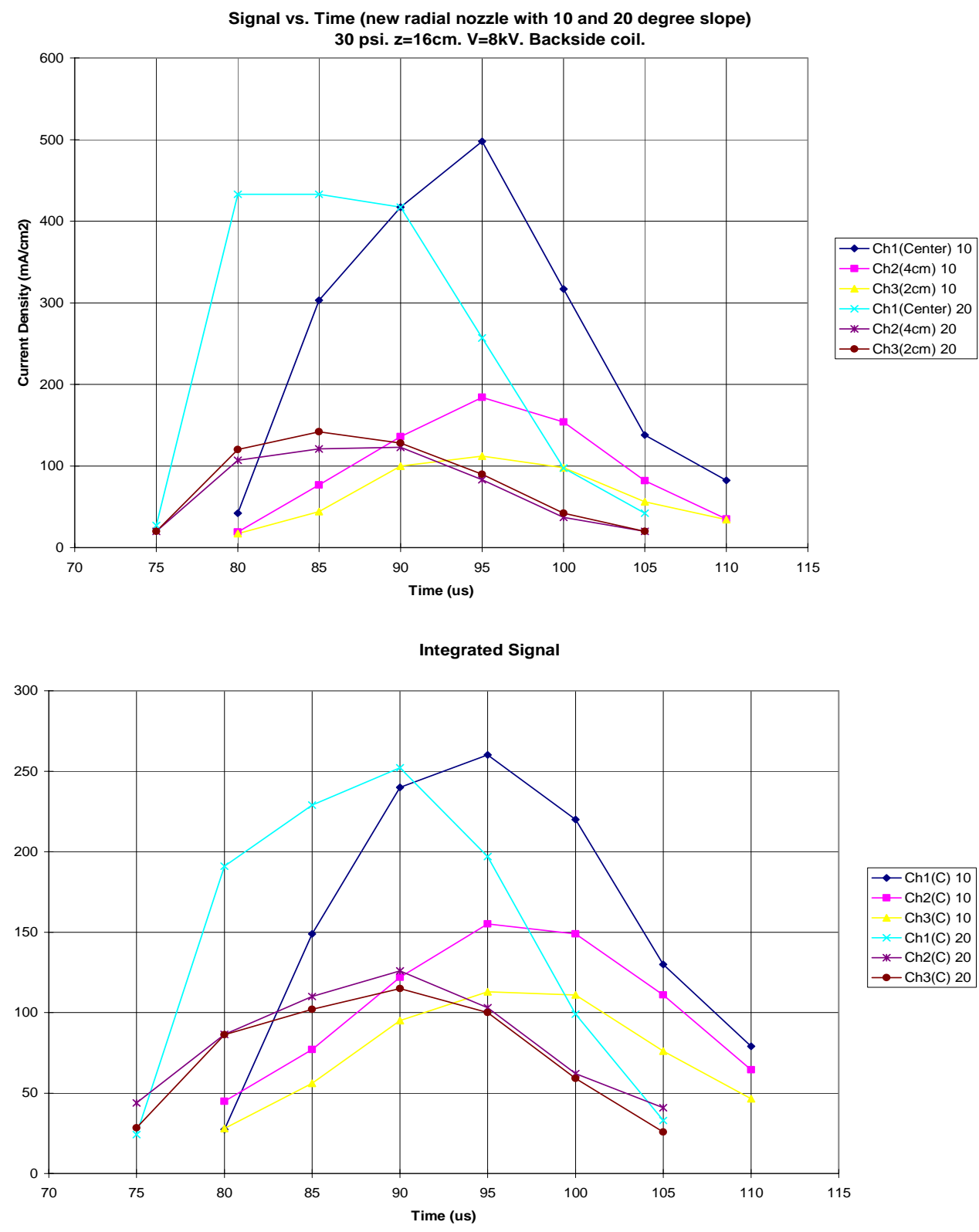

Figure 2.3.2. Peak and Integrated Ion Flux For 10 and 20 Degree Nozzles at Different Delay Times.

Both the peak ion flux and the integral increases as the ionization coil driver voltage is increased. This is seen in Figure 2.3.3, with the Faraday cup located $16 \mathrm{~cm}$ from the 
source. Up to $9 \mathrm{kV}$, the maximum voltage possible with our present driver, the ion flux is still increasing with increased voltage.

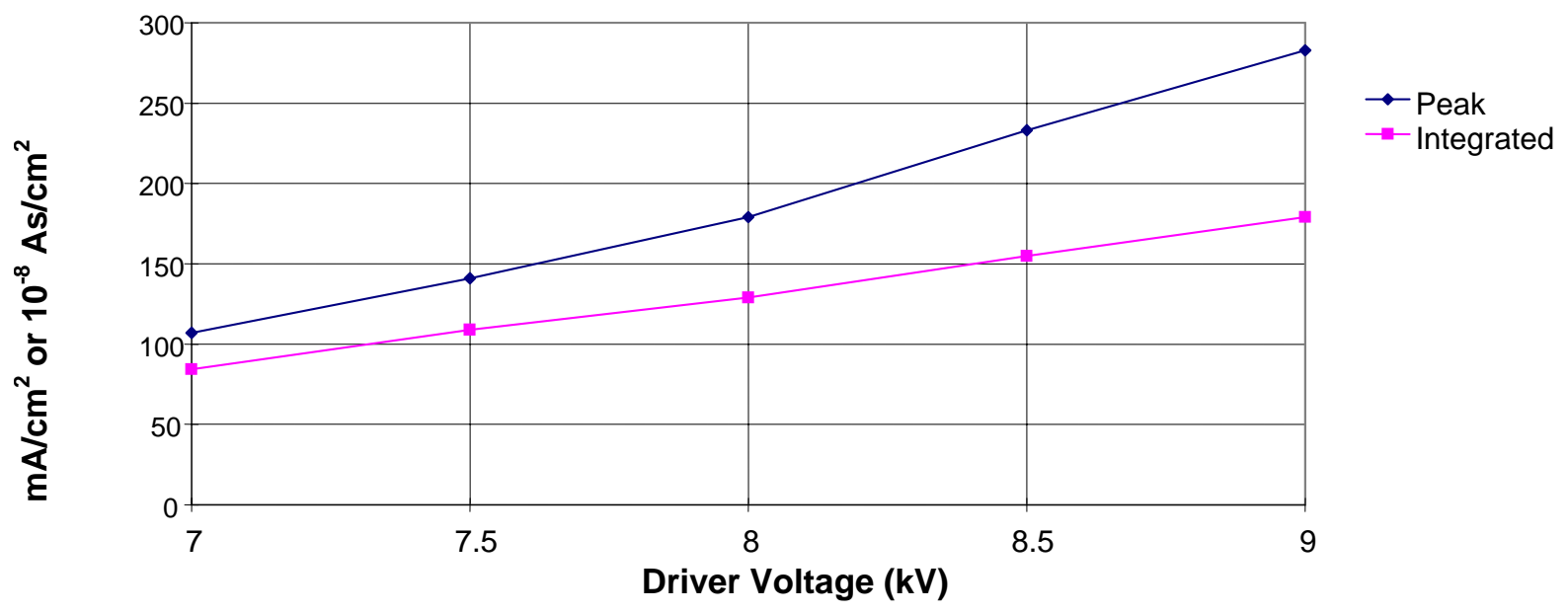

Figure 2.3.3. Ion Flux (peak and integrated) $16 \mathrm{~cm}$ from the Source as a Function of Driver Voltage.

\subsubsection{Reproducibility}

The reproducibility of the source was observed by operating at a 2 pulse per minute repetition rate. The digital oscilloscopes were set to operate in the envelope mode, wherein the maximum and minimum values of the signal at each time interval are stored. Irreproducibility could occur as a result of surface contamination of the plastic surfaces on the coil forms from the untrapped oil diffusion pump and other sources. Other sources of irreproducibility include variations in the performance of the high speed valve, and/or the valve and coil drivers and changes in the valve plenum pressure. Results are shown in Figure 2.3.4 for a 14-pulse sequence. These data are for operation at $8 \mathrm{kV}$ with a $100 \mu \mathrm{s}$ delay time. Similar results were obtained with the driver operated at different voltages. The upper and lower limits of the envelope of multiple signals are actually dominated by the amplitude of the noise on any one signal, so a more sophisticated measurement technique will be necessary in order to quantify reproducibility below the approximately $5 \%$ level in these envelope data. 

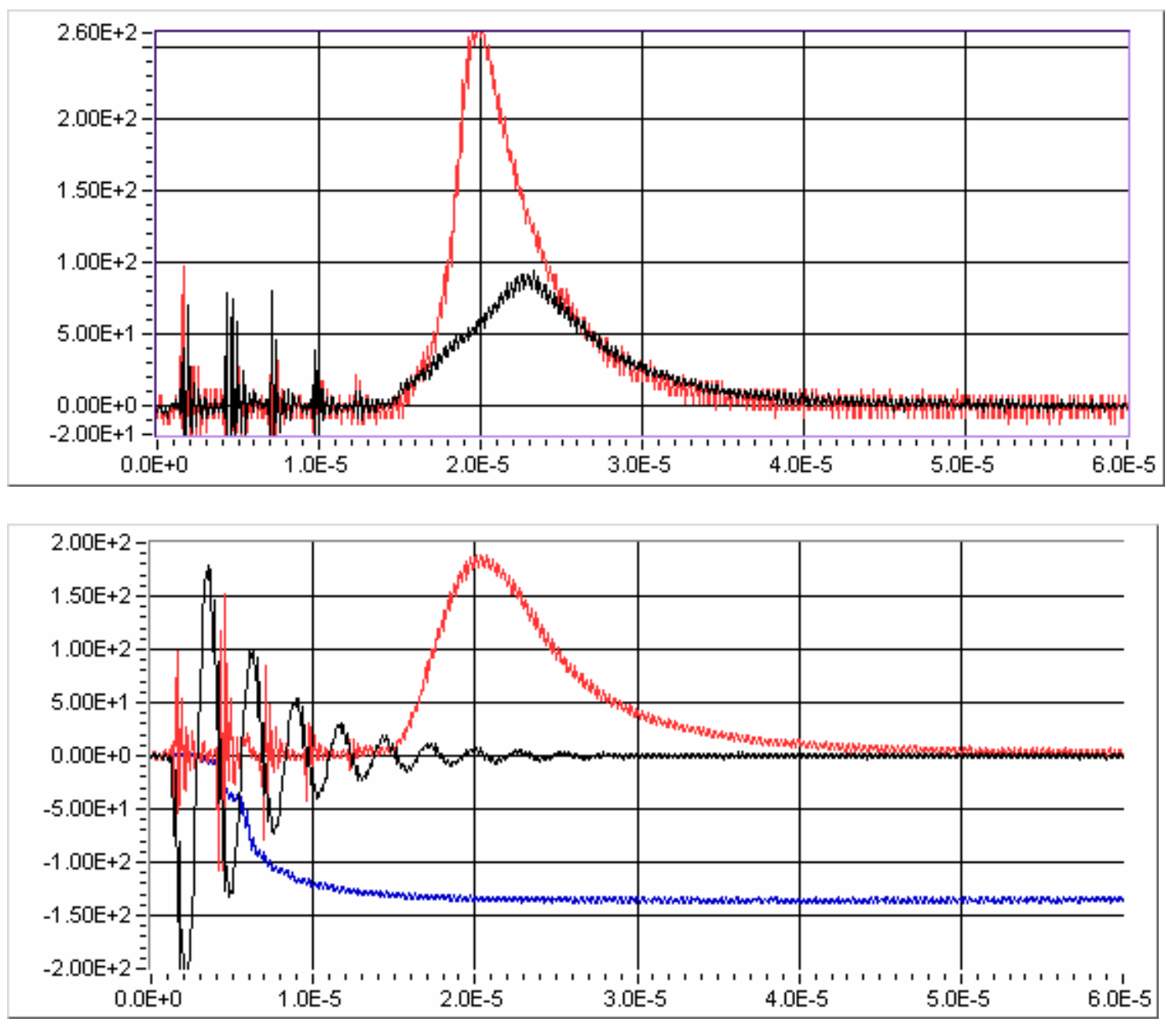

Figure 2.3.4 Superimposed Data from a 14-Shot Sequence. Upper traces are the Faraday cup signals $\left(\mathrm{mA} / \mathrm{cm}^{2}\right)$ at $\mathrm{z}=16 \mathrm{~cm}$ and $\mathrm{r}=0$ and $2 \mathrm{~cm}$. Lower traces are driving current (500 A/div), PMT signal (arb. units) and Faraday cup $\left(\mathrm{mA} / \mathrm{cm}^{2}\right)$ at $\mathrm{z}=16 \mathrm{~cm}$ and $\mathrm{r}=4 \mathrm{~cm}$.

\subsubsection{Azimuthal Uniformity}

The purpose of the Phase I uniformity measurements was to determine the extent of nonuniformity and to identify major sources contributing to non-uniformity. Optimizing the source in this regard will be undertaken in Phase II.

The azimuthal uniformity was measured using an array of Faraday cups that could be rotated about the axis. Measurements were made at 90 degree intervals. The peak Faraday cup signal and the integral of this signal for cups $16 \mathrm{~cm}$ from the source at 2 and $4 \mathrm{~cm}$ radii are plotted in Figure 2.3.5. As can be seen, there is a substantial azimuthal non-uniformity. The ratio of the maximum to minimum signal is $\sim 2.2$. This ratio decreases to 1.6 for measurements made $32 \mathrm{~cm}$ from the source. This non-uniform pattern is highly reproducible, at the same level as seen in Figure 2.3.4. 
Much of the non-uniformity is due to the performance of the high speed valve and the size of its plenum. Rotating the valve 90 degrees also shifts the angular location of the maximum and minimum signals, as can be seen in Figure 2.3.6.
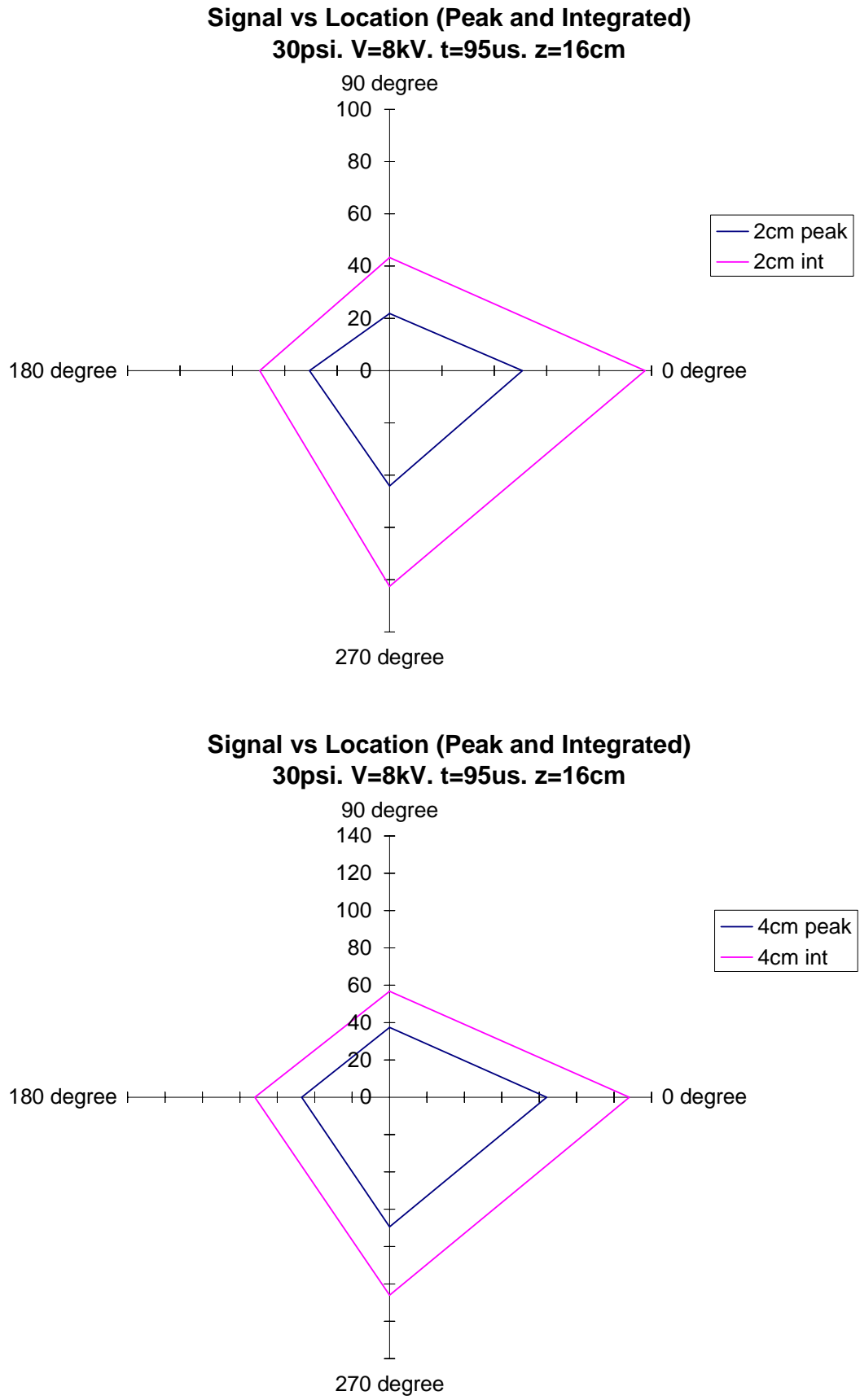

Figure 2.3.5. Azimuthal Uniformity at Radii of 2 and $4 \mathrm{~cm}$. Data was measured at 4 angular locations. Peak and integrated values of the signal are plotted. 


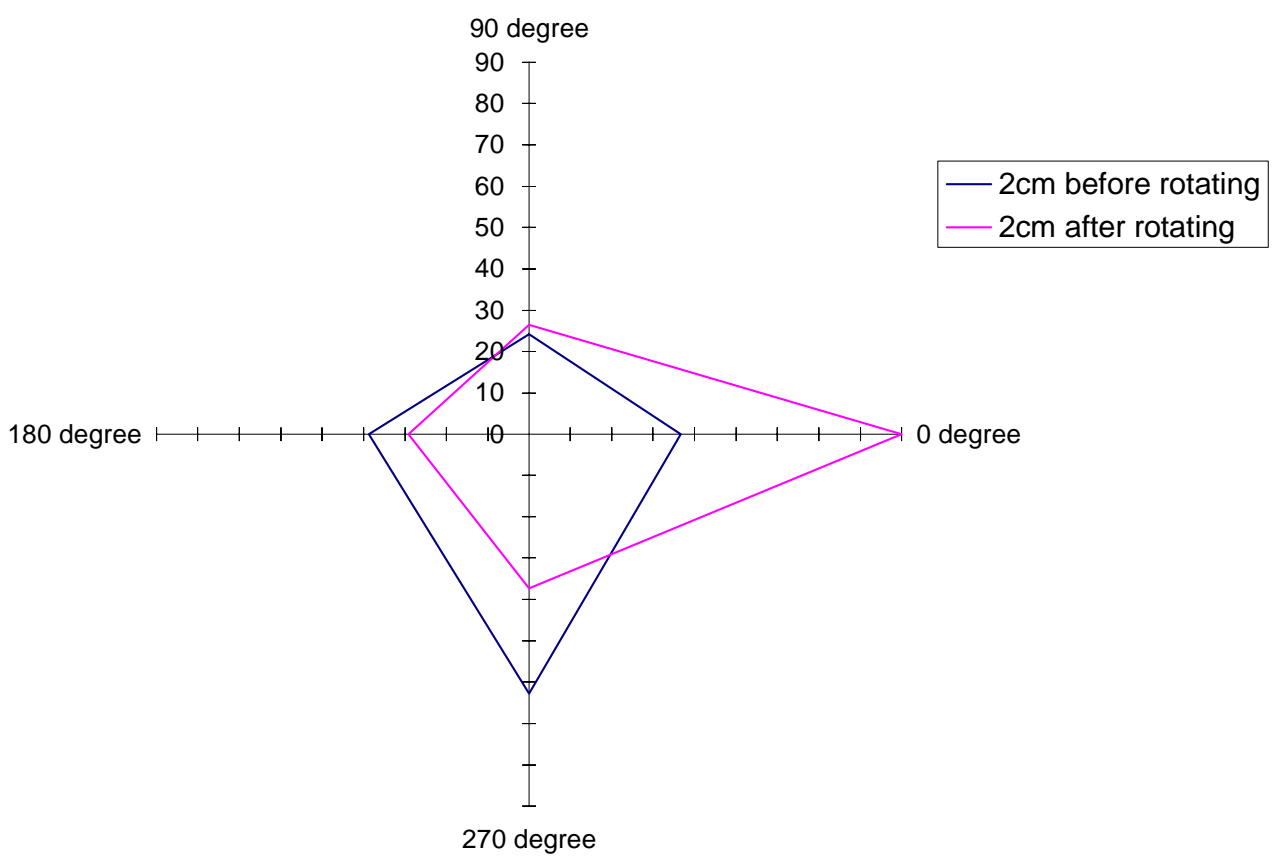

Figure 2.3.6. Effects of Rotating the High Speed Valve 90 Degrees Counterclockwise. Data are peak signal size at $\mathrm{r}=2 \mathrm{~cm}, \mathrm{z}=16 \mathrm{~cm}$, expressed as a percentage of the center Faraday cup signal.

The degree of non-uniformity is increased by the manner in which we are using the valve. Fast ionization gauge measurements indicate a $5 \mu \mathrm{s}$ spread in the opening time of the valve at different azimuthal locations. This has been observed with other valves in the past as well. As discussed earlier, the maximum ion flux occurs when the gas density is relatively low. At higher densities, collisions with the neutral gas prevent ions from leaving the source. Because of the relatively large plenum volume, this density is reached very near the beginning of the gas pulse. At this time, the ratio of the minimum and maximum density around the circumference of the valve is greatest because of the spread in opening time. Using a smaller plenum size would permit operation closer to the peak of the gas pulse, where this ratio is much smaller.

Changing the delay time between the high speed valve and ionization coil drivers demonstrates the effects of the changing gas density on ion flux output. Figure 2.3.7 shows the peak Faraday cup signals for different delay times at 4 different azimuthal locations. Also shown is the relative opening time of the gas valve at each of these locations. It can be seen that at early delay times the Faraday cup signals are greater at the angular positions ( 0 and 270) where the gas arrives first. The longer delay times result in the shift of the peak signals to the other angular locations (90 and 180) as gas begins to appear there, while at the previous locations the gas density has increased to the level that collisions with the ions begin to decrease the ion flux. 


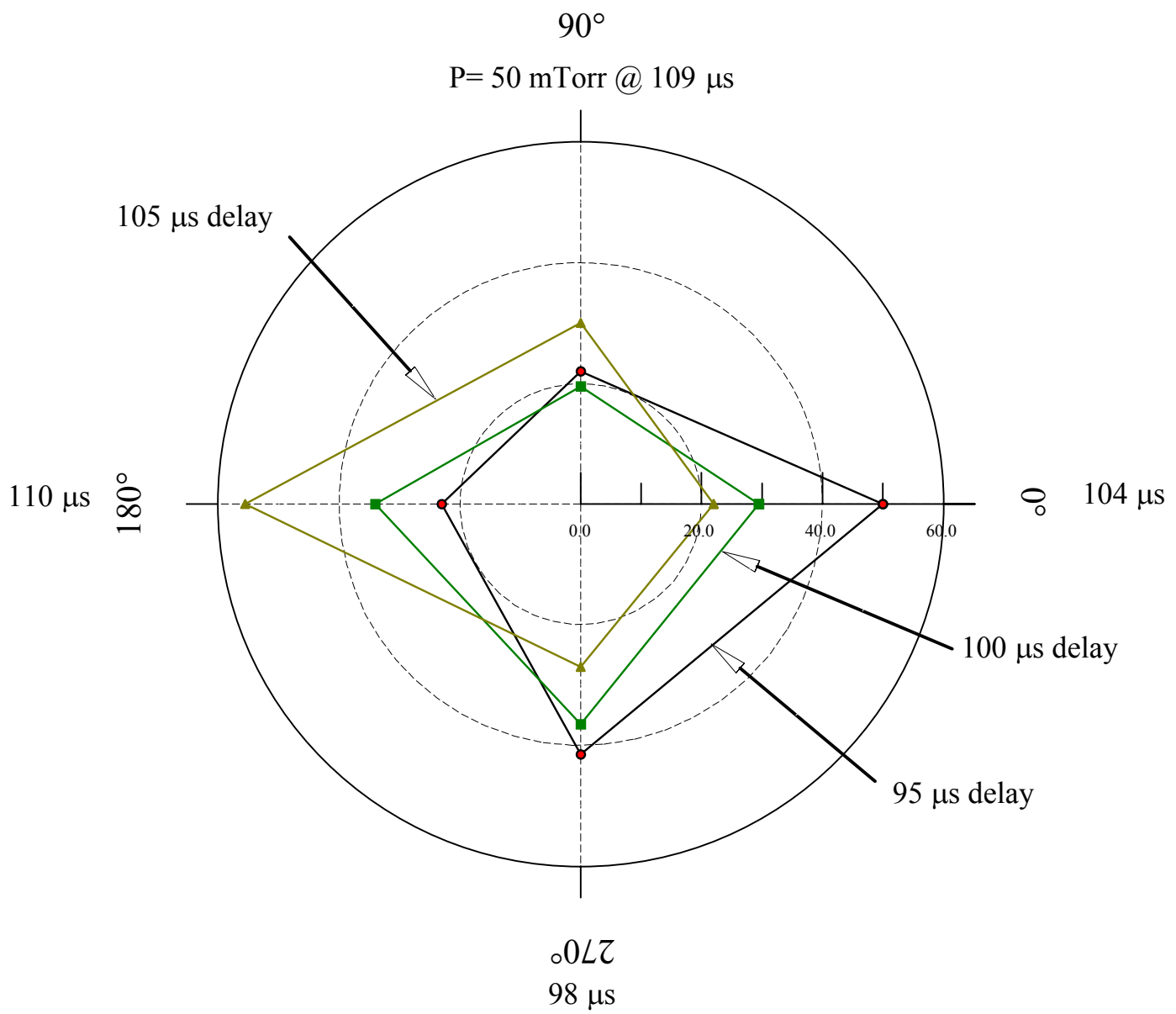

Figure 2.3.7. Relative Peak Faraday Cup Signals for Delay Times of 95, 100 and $105 \mu$ s. The time interval from the triggering of the gas valve until the gas pressure has reached $50 \mathrm{mT}$ Trr at a radius of $4 \mathrm{~cm}$ is indicated for each 90 degree location.

\subsubsection{Radial Distribution}

The ion flux is a function of radius and distance $(8-28 \mathrm{~cm})$ from the source is shown in Figure 2.3.8. 
Applied Pulsed Power, Inc.

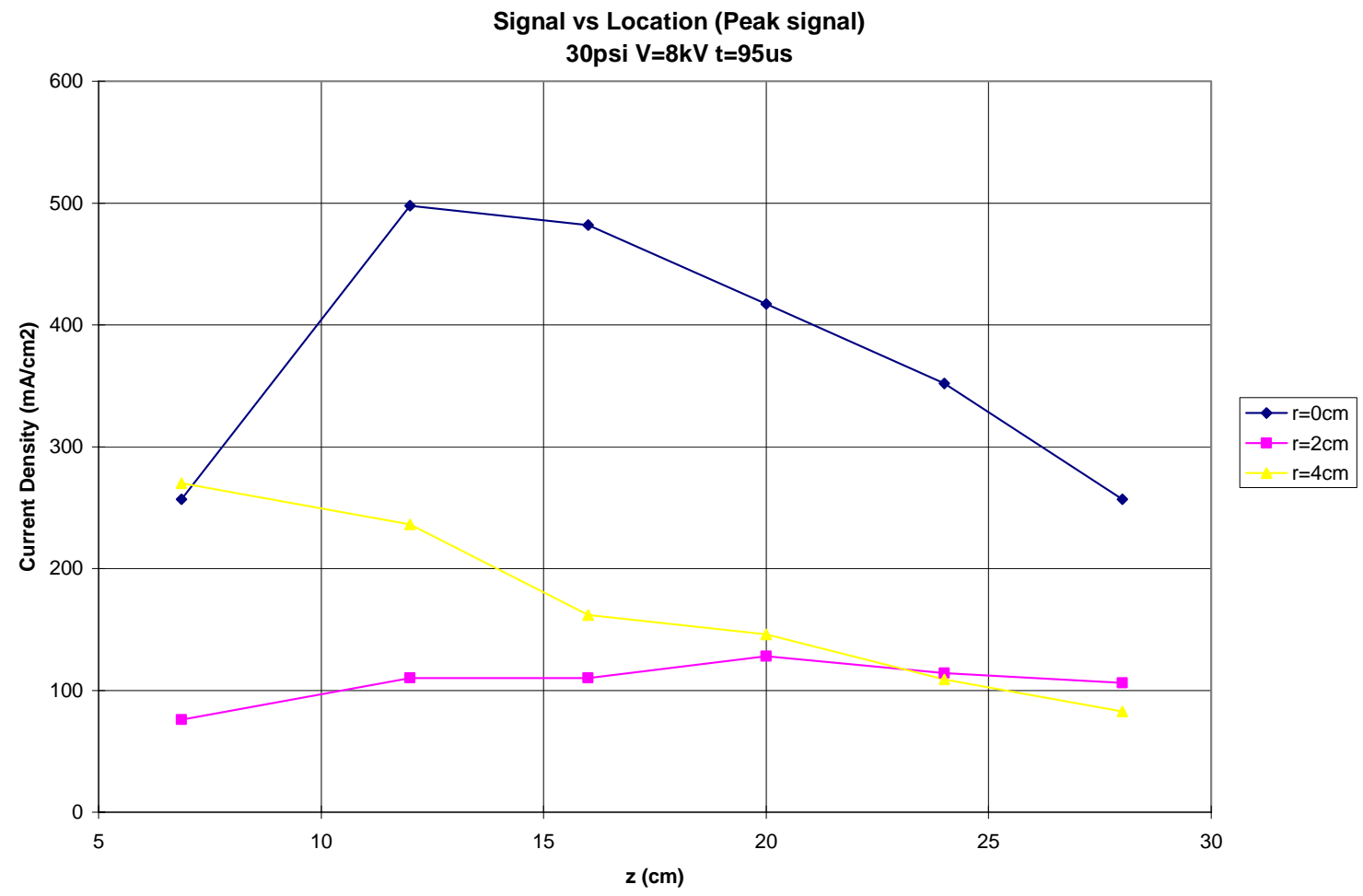

Signal vs Location (Integrated signal) $30 p s i \mathrm{~V}=8 \mathrm{kV} t=95 \mathrm{us}$

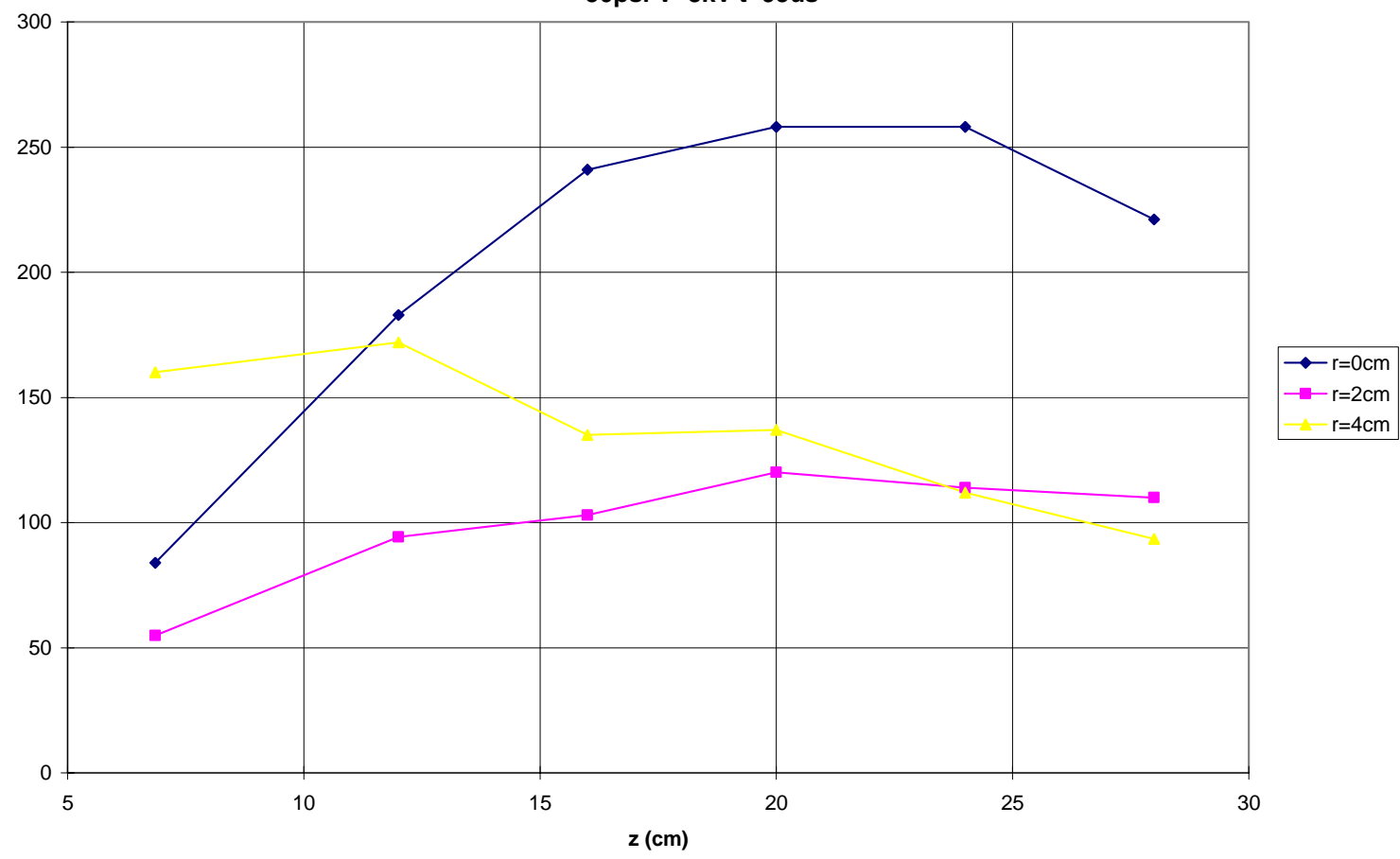

(b) Integrated Faraday Cup Signal

Figure 2.3.8. Radial Ion Flux Distribution as a Function of Distance from the Source. 
The relative ion flux as a function of radius was also found to be dependent on the driving voltage. This can be seen in Figure 2.3.9 which compares Faraday cup measurements at 0 and $2 \mathrm{~cm}$ for different driver voltages. At $9 \mathrm{kV}$, the signals are very similar.

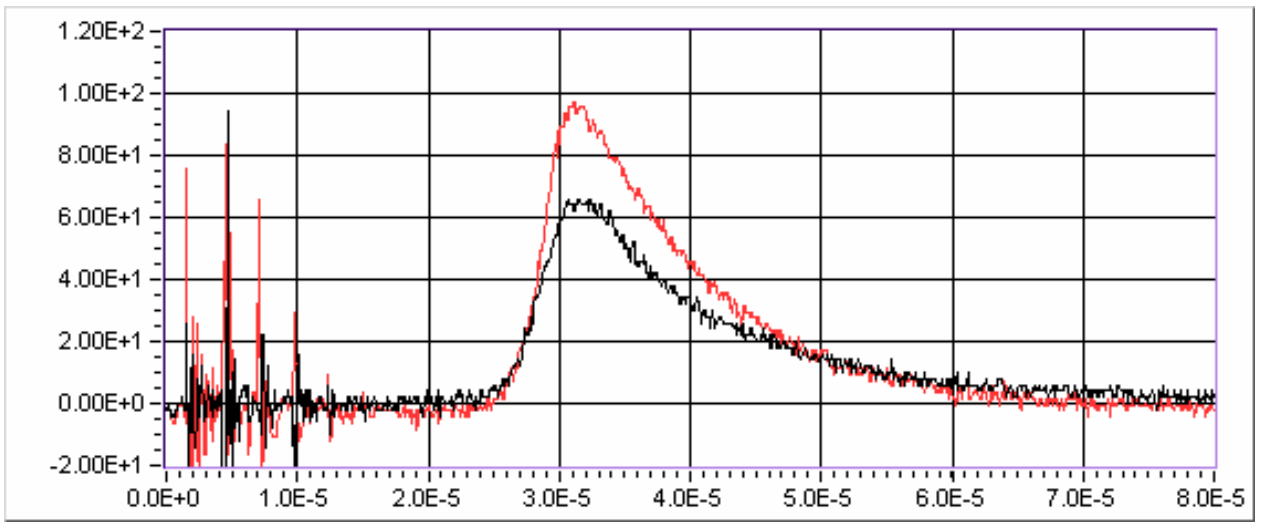

(a) $7 \mathrm{kV}$ driver voltage. 461

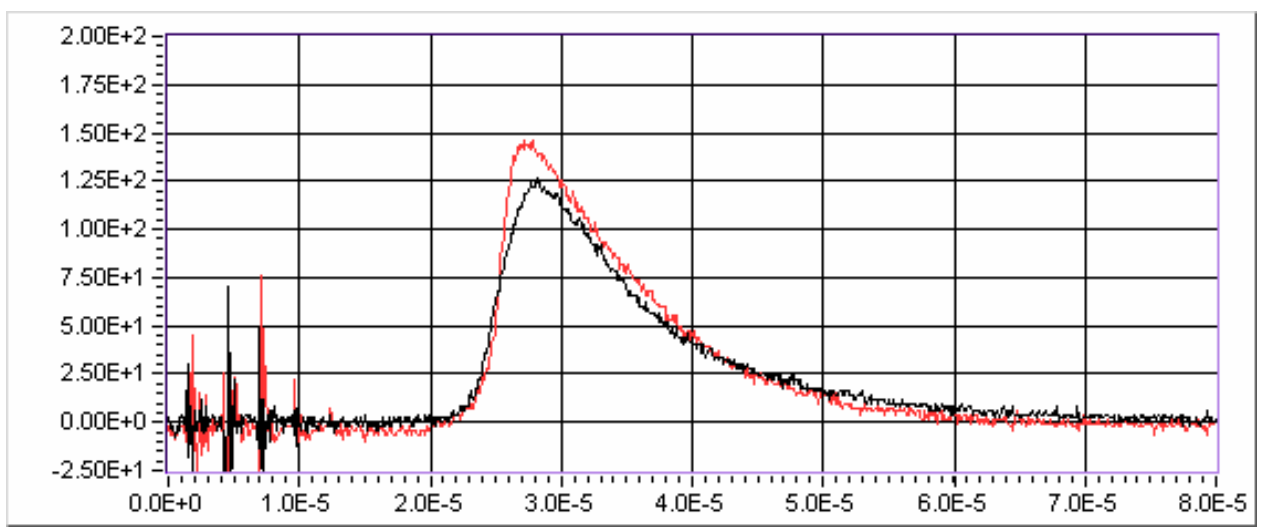

(b) $8 \mathrm{kV}$ driver voltage. 455

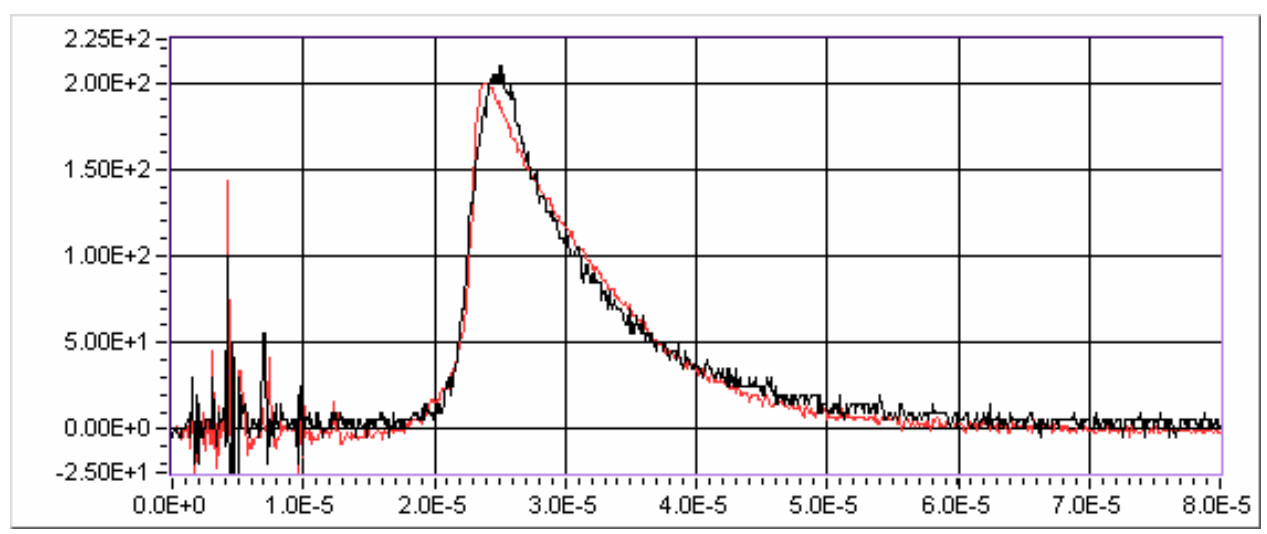

(c) $9 \mathrm{kV}$ driver voltage 462

Figure 2.3.9. Comparison of Ion Flux Measured $32 \mathrm{~cm}$ from Source at Radii of 0 and 2 $\mathrm{cm}$ for Different Ionization Coil Driver Voltages. 
It should also be possible to alter the magnetic field geometry of the source to adjust the radial distribution, but this was not attempted in Phase I.

\subsubsection{Effects of Driving Waveforms on Source Performance}

Experiments were undertaken to determine the effects of altering the driving wave form on the output of the source.

Initially, resistance was added to the circuit to increase the amount of damping. These results are shown in Figure 2.3.10. The effects of adding even a small amount $(0.17 \Omega)$ of damping are quite dramatic. The peak amplitude of the signal decreases by a factor of $>2$. The pulse shape is also affected, resulting in a flatter and somewhat longer pulse.

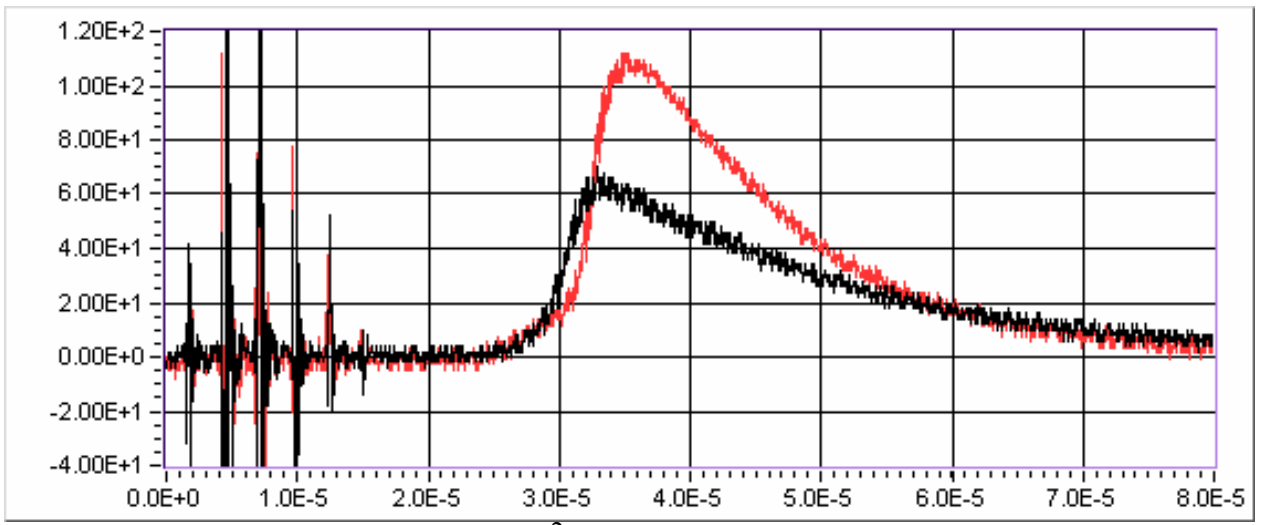

Faraday cups $\left(\mathrm{mA} / \mathrm{cm}^{2}\right)$ at $\mathrm{z}=32 \mathrm{~cm}, \mathrm{r}=0$ and $2 \mathrm{~cm}$.

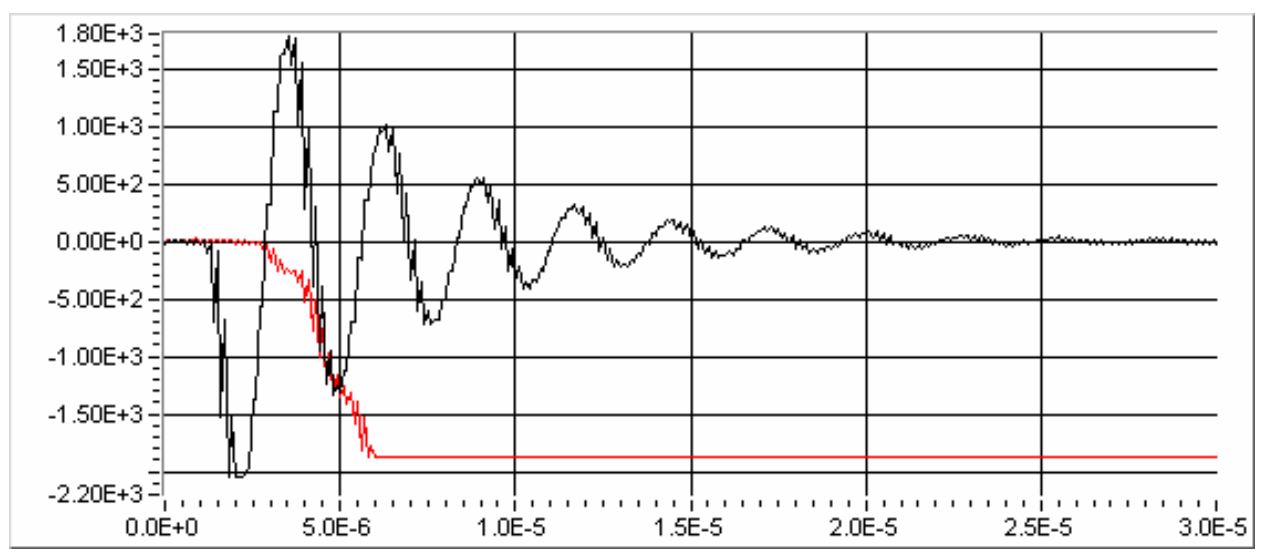

Driver current (A) and photomultiplier tube signal (x50)

Figure 2.3.10(a) No Additional Damping (Envelope for 14 Shots) 


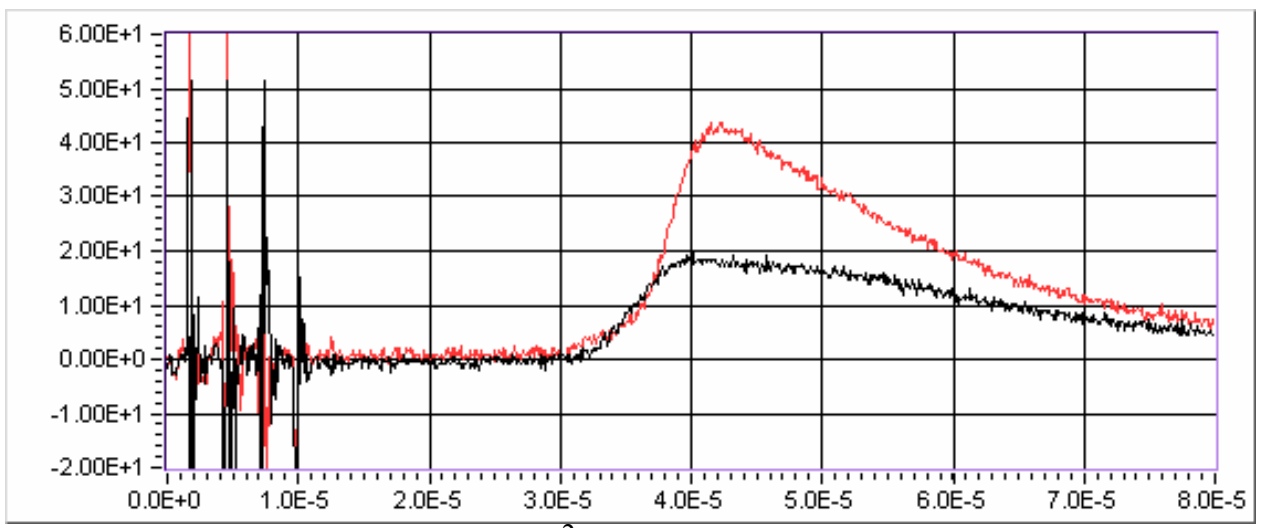

Faraday cups $\left(\mathrm{mA} / \mathrm{cm}^{2}\right)$ at $\mathrm{z}=32 \mathrm{~cm}, \mathrm{r}=0$ and $2 \mathrm{~cm}$.

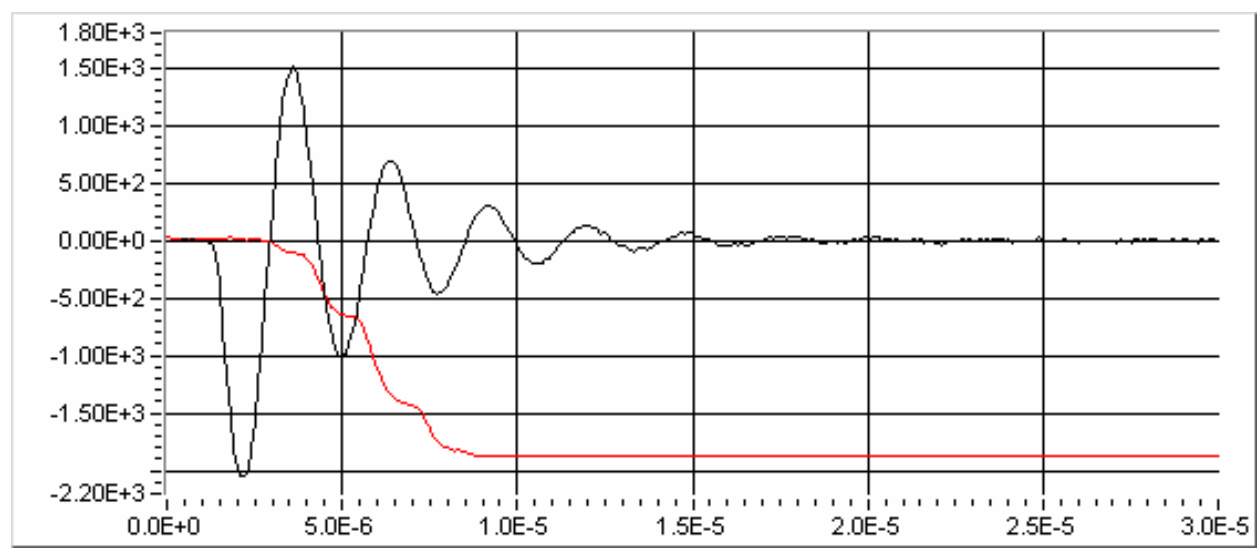

Driver current (A) and photomultiplier tube signal (x50)

(b) $0.17 \Omega$ damping

Figure 2.3.10. The Effects of Adding Damping Resistance to the Ionization Coil Driver Circuit on Source Output. All data was taken with 30 psig argon and $8 \mathrm{kV}$ driver voltage.

To pursue this observation in a simple way, a second switch was added to truncate the drive pulse after a certain number of oscillations. Some of these results are shown in Figure 2.3.11. Truncating the pulses after 4 cycles, when the drive pulse has decayed to $15 \%$ of its initial value, decreases the output by $40 \%$. Truncating after 2 cycles decreases the signal by $85 \%$.

It appears that after the initial large amplitude cycle that results in the breakdown of the gas, a much smaller drive would be sufficient to generate and accelerate the ions. By tailoring the shape of the drive pulse, it may be possible to both extend the duration and control the shape and magnitude of the ion flux pulse. 


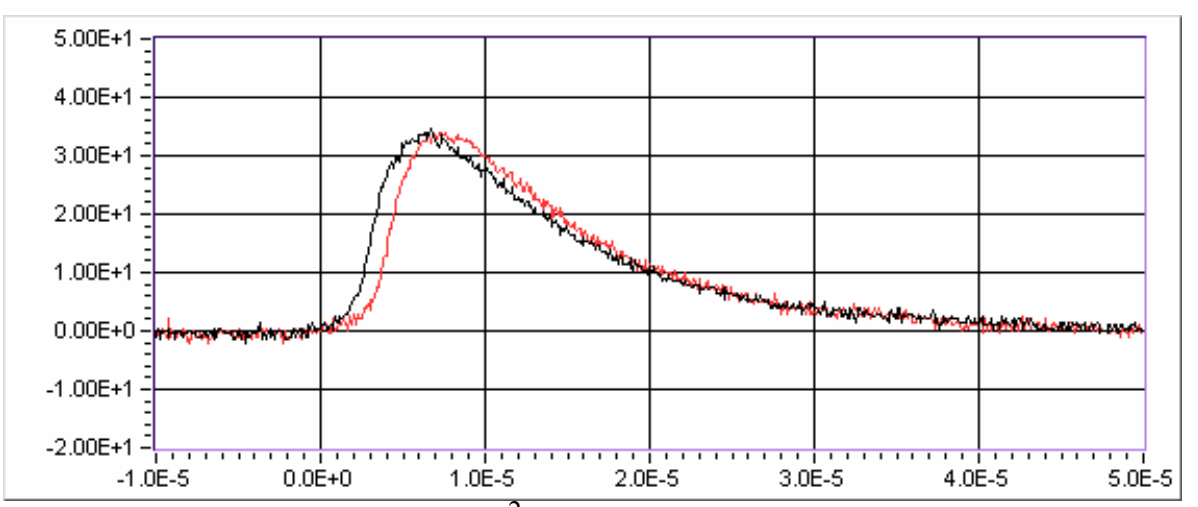

Faraday cups $\left(\mathrm{mA} / \mathrm{cm}^{2}\right)$ at $\mathrm{z}=32 \mathrm{~cm}, \mathrm{r}=0$ and $1.2 \mathrm{~cm}$.

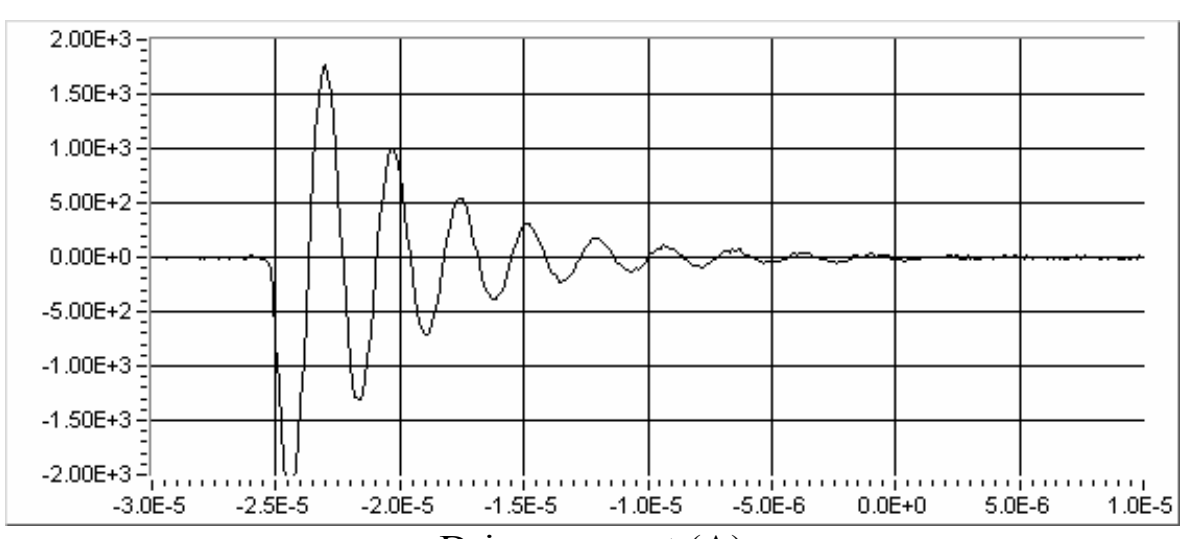

Driver current (A)

Figure 2.3.11 (a) No Truncation 


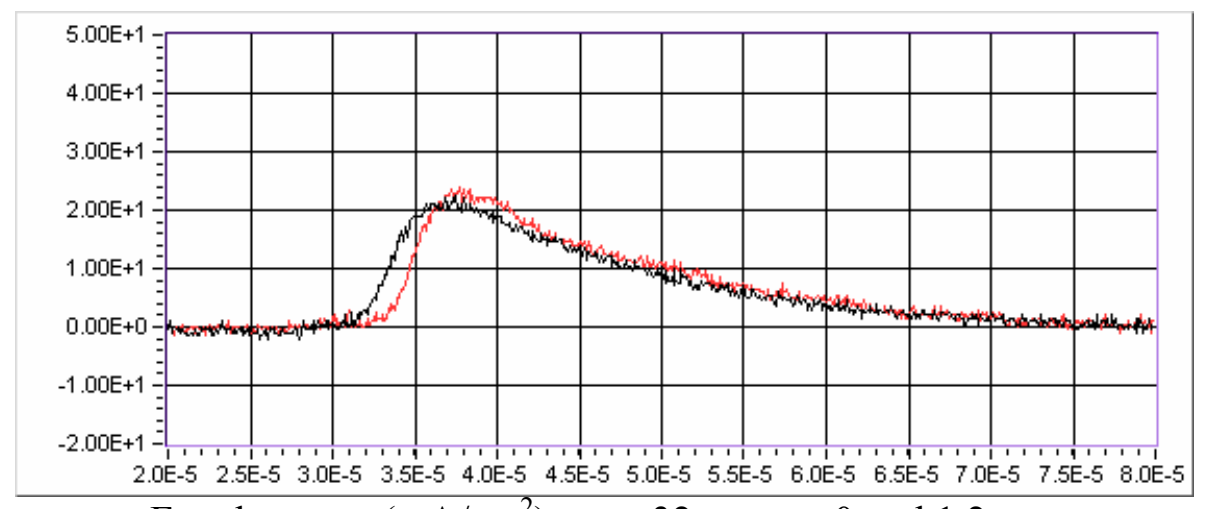

Faraday cups $\left(\mathrm{mA} / \mathrm{cm}^{2}\right)$ at $\mathrm{z}=32 \mathrm{~cm}, \mathrm{r}=0$ and $1.2 \mathrm{~cm}$.

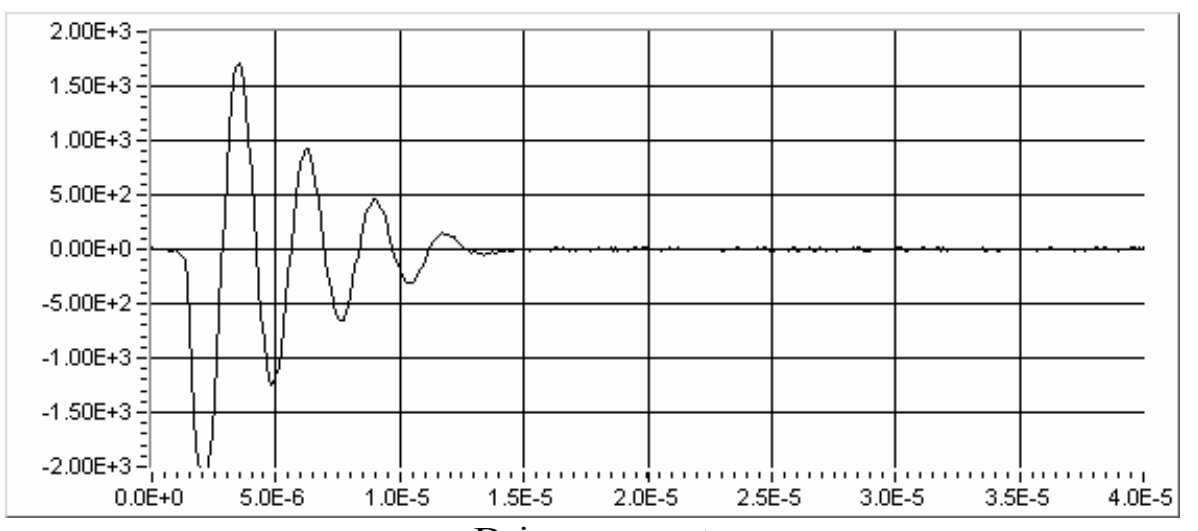

Driver current

2.3.11(b) Truncated after $\sim 4$ Cycles 


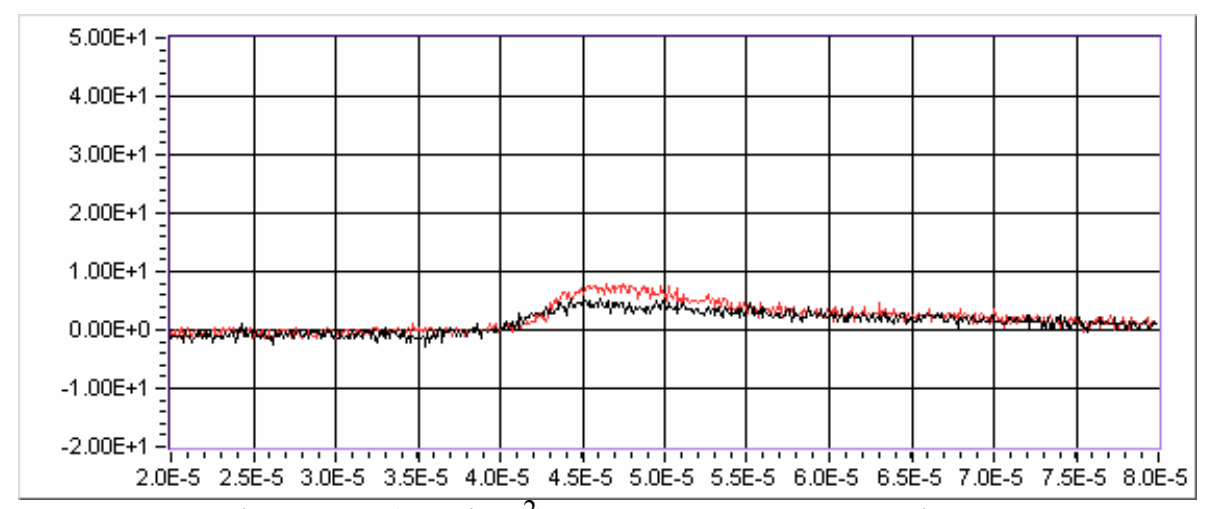

Faraday cups $\left(\mathrm{mA} / \mathrm{cm}^{2}\right)$ at $\mathrm{z}=32 \mathrm{~cm}, \mathrm{r}=0$ and $1.2 \mathrm{~cm}$.

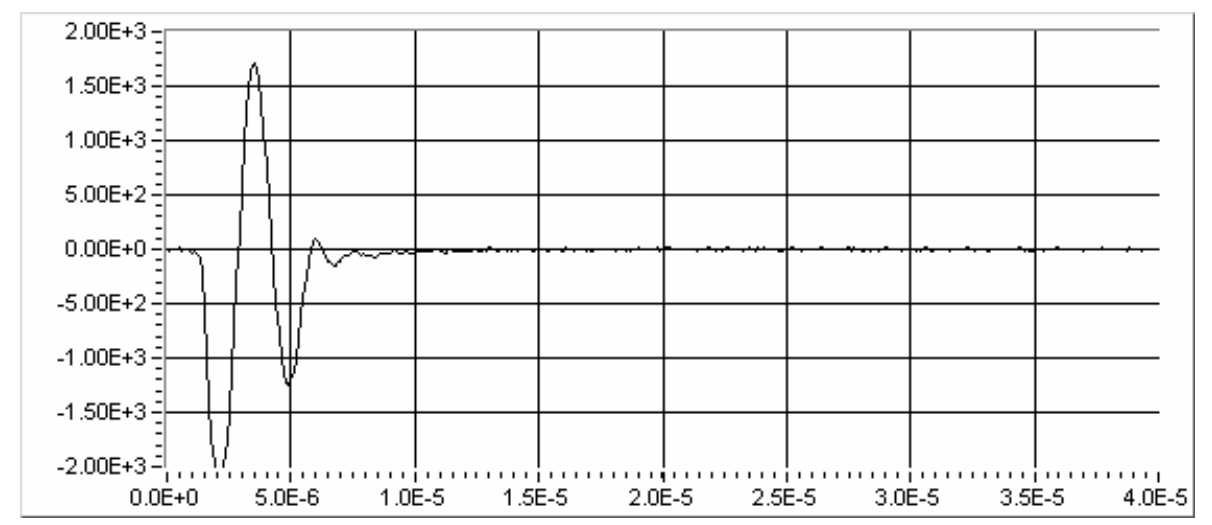

Driver current (A)

\subsubsection{1(c) Truncated after $\sim 2$ Cycles}

Figure 2.3.11 Effects of Truncating the Drive Pulse on the Output of the Ion Source. All data was taken with 30 psig argon and $8 \mathrm{kV}$ driver voltage.

\subsubsection{Plenum Pressure}

Performance of the source was examined with high speed valve plenum pressures of 3 , 1.6, and 0.7 atm. Maximum source output was achieved with a longer coil-to-valve delay time at lower plenum pressures. Also, a large output was obtained over a longer span of delay times at lower pressures. Both of these observations are consistent with our belief that collisions with neutral gas molecules attenuate the directed ion flux at longer delay times.

The magnitude of the ion fluxes obtained at optimum delay times for different plenum pressures were comparable. It is clear therefore, that the plenum volume and thus the amount of gas loading for the system could be much smaller. 


\subsubsection{Charge State Measurements}

A pulsed time-of-flight (TOF) measurement was attempted to find the charge state distribution of the plasma ions. A pair of fine grids separated by $2 \mathrm{~mm}$ was used, with $0.5-2 \mathrm{kV}$ accelerating voltage applied. The TOF was measured to a Faraday cup $16 \mathrm{~cm}$ downstream. A TOF difference of about 1 microsec would result for $\mathrm{Ar}+2$ ions relative to Ar+1. Unfortunately, the head of the plasma stream makes a smooth rise in about 5 microseconds, and just before this rise, noise from the driving circuit disturbs the baseline. Furthermore, plasma Debye shielding effects on the grids were clearly visible in the behavior of the signals with the accelerating voltage. Thus, this technique was able establish only that doubly or higher charged species could not be more than roughly $10 \%$ of the plasma ion population. A more precise measurement will require gating of the plasma or the accelerating voltage to give a clear separation of the charge species, a procedure which was to be undertaken during Phase II.

\subsection{Discussion of Results Relative to Phase I Goals and the Requirements for Heavy Ion Fusion Accelerators}

The Phase I goals were to determine the desired ion source parameters and conduct experiments to evaluate the performance of various pulsed inductive plasma source configurations. In this section we will discuss the potential of the source for heavy ion fusion applications.

The source we have investigated produces a stream of Argon plasma with approximately $80 \mathrm{eV}$ directed axial energy, and approximately $0.6 \mathrm{eV}$ transverse ion temperature. The ion current density is above $100 \mathrm{~mA} / \mathrm{cm}^{2}$, with different levels available at varying operating conditions. The ion density is thus $\sim 3 \times 10^{13} / \mathrm{cm}^{3}$. The source has produced fairly uniform ion current density into a $4 \mathrm{~cm}$ diameter circle, and has total pulse length $>10$ microseconds.

Two features of the data stand out relative to the stringent requirements for a HIF ion source. First, the inductive source shows very reproducible performance under a wide variety of operating conditions, the result of the statistics of the gas breakdown as driven by the inductive electric field, using the preionization method we have described. The level of this reproducibility remains to be accurately determined, since it is better than the present diagnostics can resolve at the $5 \%$ level.

Second, several means have been identified with the potential to control and optimize both the radial and temporal uniformity of the plasma stream from this source. The Phase I version of the source has substantial spatial non-uniformity and a peaked temporal pulse shape, but since the spatial and temporal shapes are highly reproducible, these means of control can readily be used in practice. During the brief Phase I investigation it was observed that simple changes in driving waveform can improve both radial uniformity and temporal shape. These observations imply that with careful choice of driving pulse shape and driving coil configuration, both radial uniformity and temporal pulse shape can 
be adjusted to meet the requirements for HIF. This optimization is a prime objective of Phase II.

Clearly, the azimuthal non-uniformity observed in the present source is caused by a nonuniform supply of gas from the fast valve to the ionization region. Possible means of reducing this non-uniformity are described in the Phase II work plan.

The results of the Phase I studies allow comparison of the pulsed, streaming source with other sources that have been investigated for HIF. In comparison with the $\sim 0.1 \mathrm{eV}$ surface ionization sources presently used, our source provides an order of magnitude higher current density and reduced gas load; also, the gas produced is argon instead of potassium, which is more benign in its effects on the accelerator structure. Surface ionization sources are not capable of reaching the high current density $(>100 \mathrm{~mA} / \mathrm{cm} 2)$ regime that enables use of a more compact injector structure. The pulsed streaming source clearly meets this criterion. The transverse ion temperature of $\sim 0.6 \mathrm{eV}$ is higher than for the surface sources, but this is tolerable at the higher current density (1).

The comparison with RF sources in terms of ion optics will be discussed in the beam extraction and acceleration tasks below. It should be noted that the risetime of the pulsed streaming source is somewhat faster than thermal RF sources, possibly allowing better control of the head of the extracted beam pulse. The gas load is smaller due to the pulsed gas valve of the streaming source, which eases pumping requirements and allows lower pressure in the injector optics, thus reducing charge exchange and other deleterious effects on the beam. 


\subsection{Phase II Source}

\subsection{Description of Phase II Source}

\subsubsection{Mechanical Structure of the Phase II Source}

The Phase II source was modified to take advantage of the possibilities for improved performance identified by the results of Phase I. The Phase I source used an APP designed fast gas injection valve to supply an annular ring of gas in front of a set of driver coils. These driver coils would both break down the gas to form a plasma, and accelerate the plasma. The Phase I source also used an additional coil concentric and co-planar with the driver coils, called a pre-ionizer, which assisted in the breakdown of the gas into plasma by providing additional free electrons in the gas during the breakdown.

The first important change in the Phase II source is a new fast gas valve. The Phase I gas valve used a pulsed magnetic field to move an annular metal ring off an o-ring, allowing an annular gas pulse to move radially out across the driver coils. The annular uniformity of this valve proved to be insufficient. The fast gas valve was modified to an axial valve designed by APP that resulted in better reproducibility and uniformity. Figure 3.1.1 shows the design of the new valve. Additional information about this valve design is provided by Appendix [patent]. 


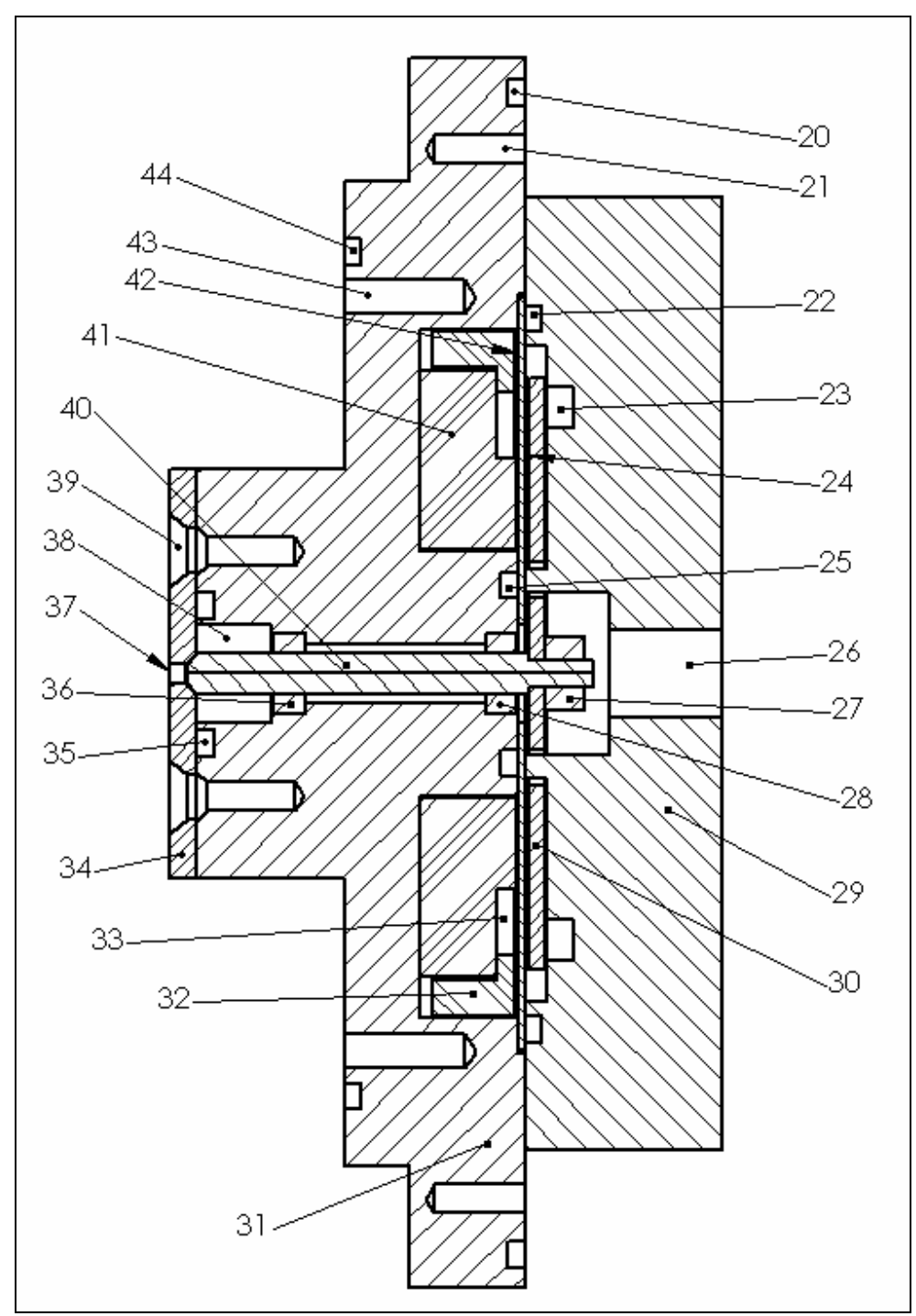

Figure 3.1.1: Phase II Fast Gas Valve

A result of switching from the original valve with an annular gas exit to this new type with axial gas exit is that the gas no longer moves preferentially in the radial direction. To properly direct the gas distribution, a nozzle structure was added at the exit of the new valve. This nozzle was in the form of a cup facing the plane of the driver coils leaving only a small gap between the cup and the plane of the driver coils. The gas flows from the valve exit axially into the cup, where it impinges on a blocking surface (13 in Figure 3.1.2). Gas then flows radially out through the gap between the cup and the coil plane to form the desired distribution above the coils. This configuration eliminates the major difficulty of the Phase I annular valve, namely the need to produce very uniform opening of the valve around the azimuthal direction. In the new version, the gas enters the nozzle structure on axis and is redirected into a radially spreading flow. The final degree of azimuthal uniformity is determined mostly by the dimensional accuracy of the nozzle structure and the gap though which the gas exits to the coil region, and is very insensitive to the opening characteristics of the valve itself. Figure 3.1.2 shows a side view of the source with the new nozzle structure. This Figure also shows the modulator (the plate 6 
with coils 7,8 at the left side of the Figure) which will be discussed in more detail in section 3.2.

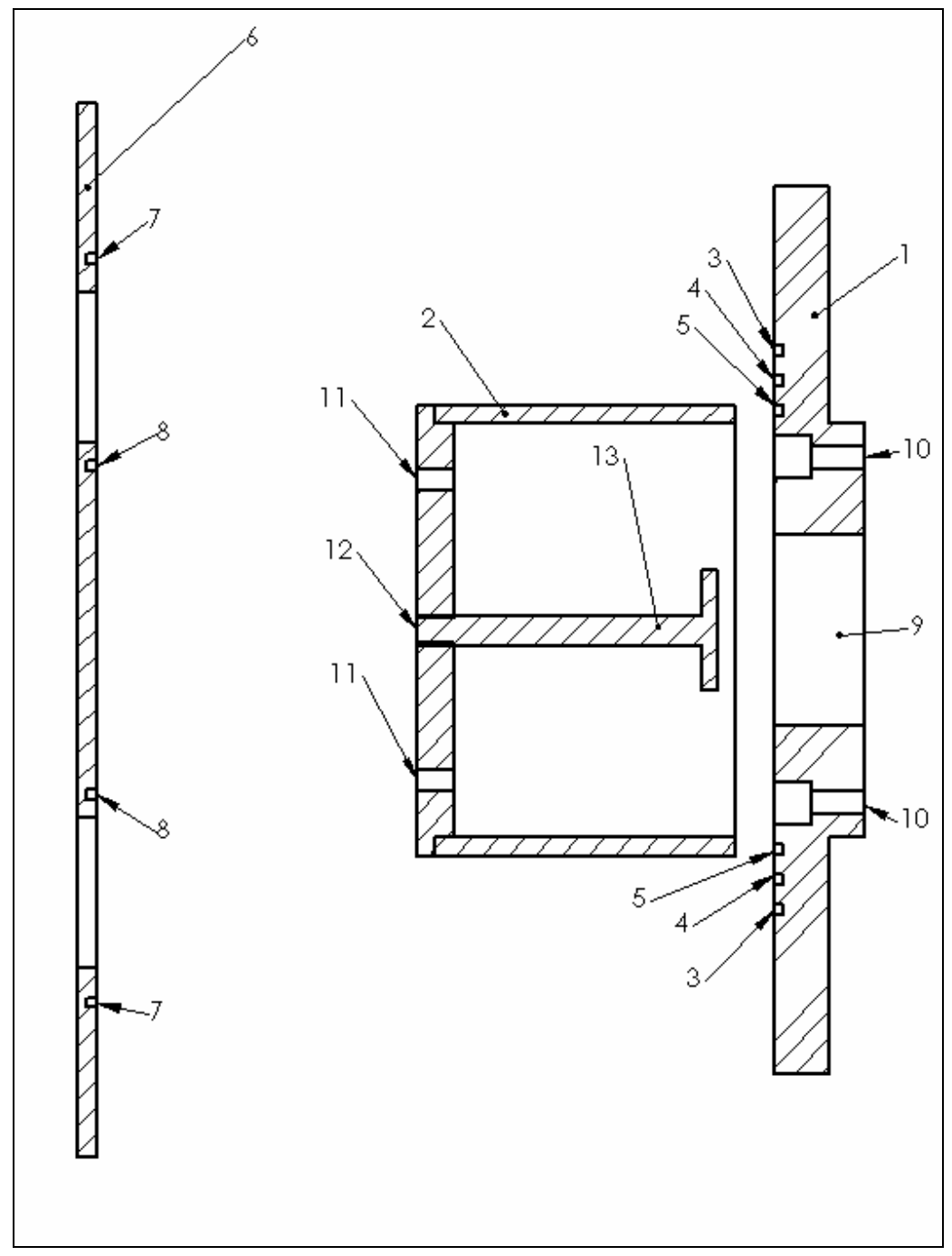

Figure 3.1.2: Side View of Source

Figure 3.1.3 shows the configuration of the source for Phase II. Coils 3 and 4 are the driver coils with feedthroughs to the pulsed power located at 91 and 92.9 is the gas source. Coil 5 is the pre-ionizer with a feedthrough at 102 and a spark gap at 90 . As can be seen in figure 3.1.2, the pre-ionizer loop is at the same diameter as the edge of the nozzle while the driver coils are at a larger diameter. 


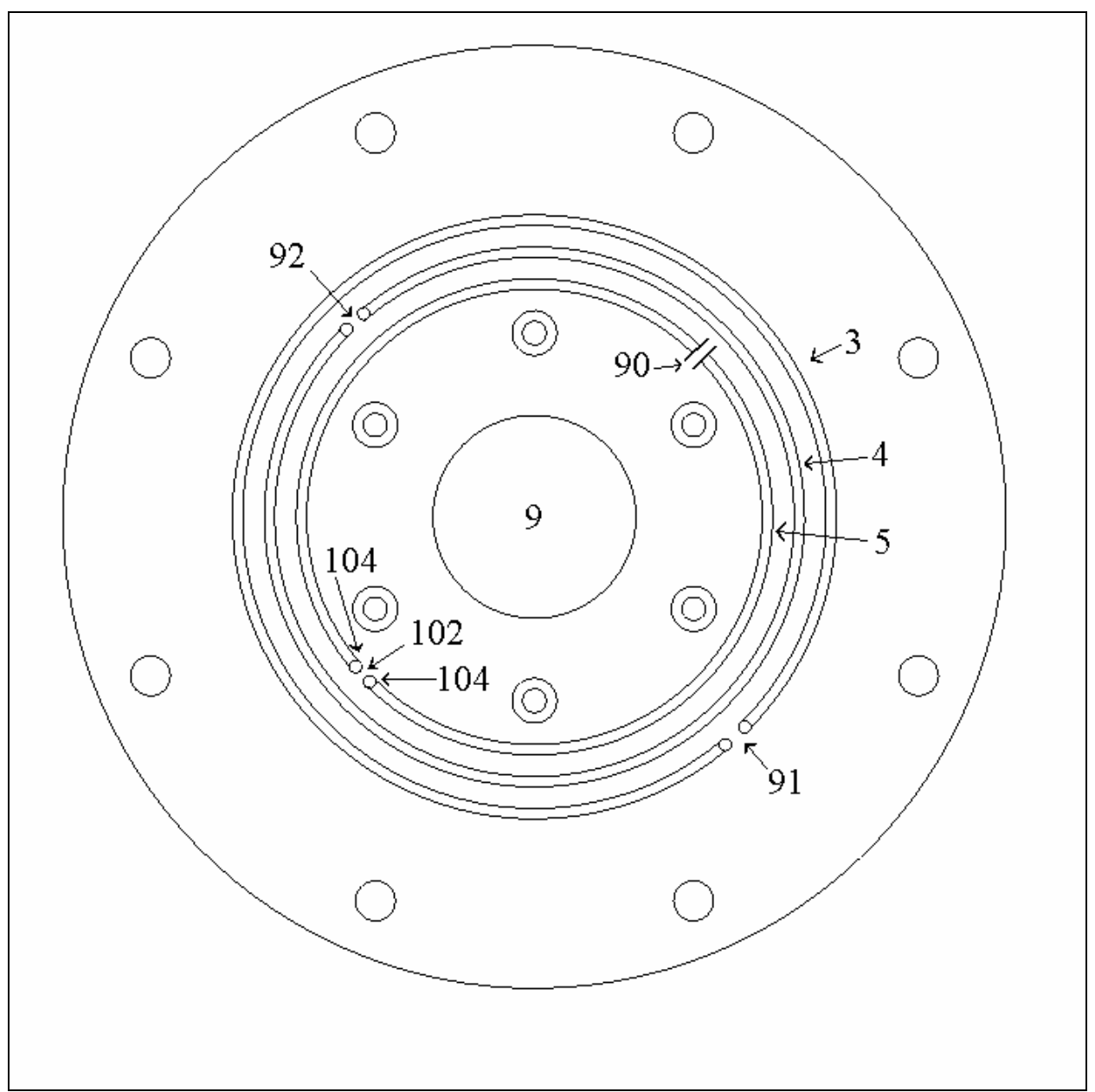

Figure 3.1.3: Driver Coil Configuration

\subsubsection{Electrical Structure of the Phase II Source}

The pulsed power to the driver coils was modified with the addition of a tank circuit located in close proximity to the source. Figure 3.1.4 shows the configuration of the pulsed power for the drive coils. The tank circuit consists of a capacitor with a low inductance connection to the driver coils. This capacitance is specifically chosen, based on the inductance of the tank circuit, to produce an oscillation period equivalent to twice the oscillation period of the initial tank circuit charging pulse. The resultant current through the driver coils can be seen in figure 3.1.5. 


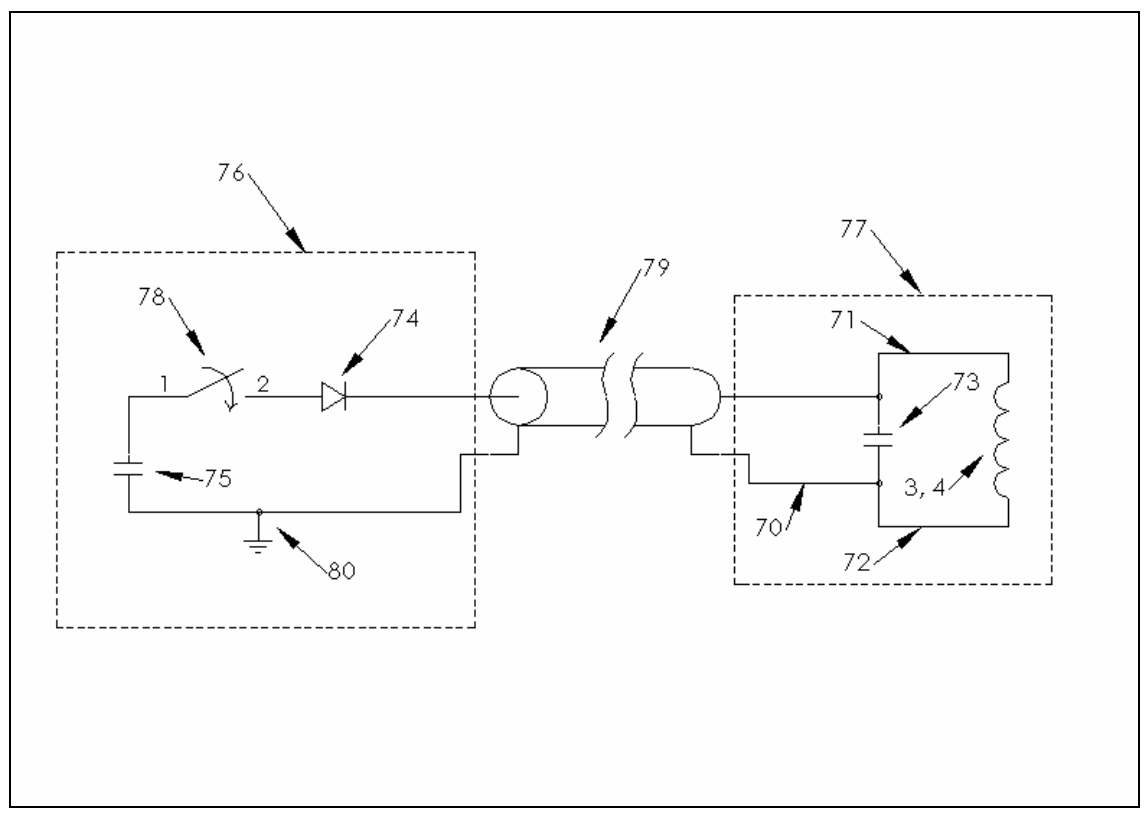

Figure 3.1.4: Driver Pulsed Power

\subsubsection{ICCD Images of Phase II Source}

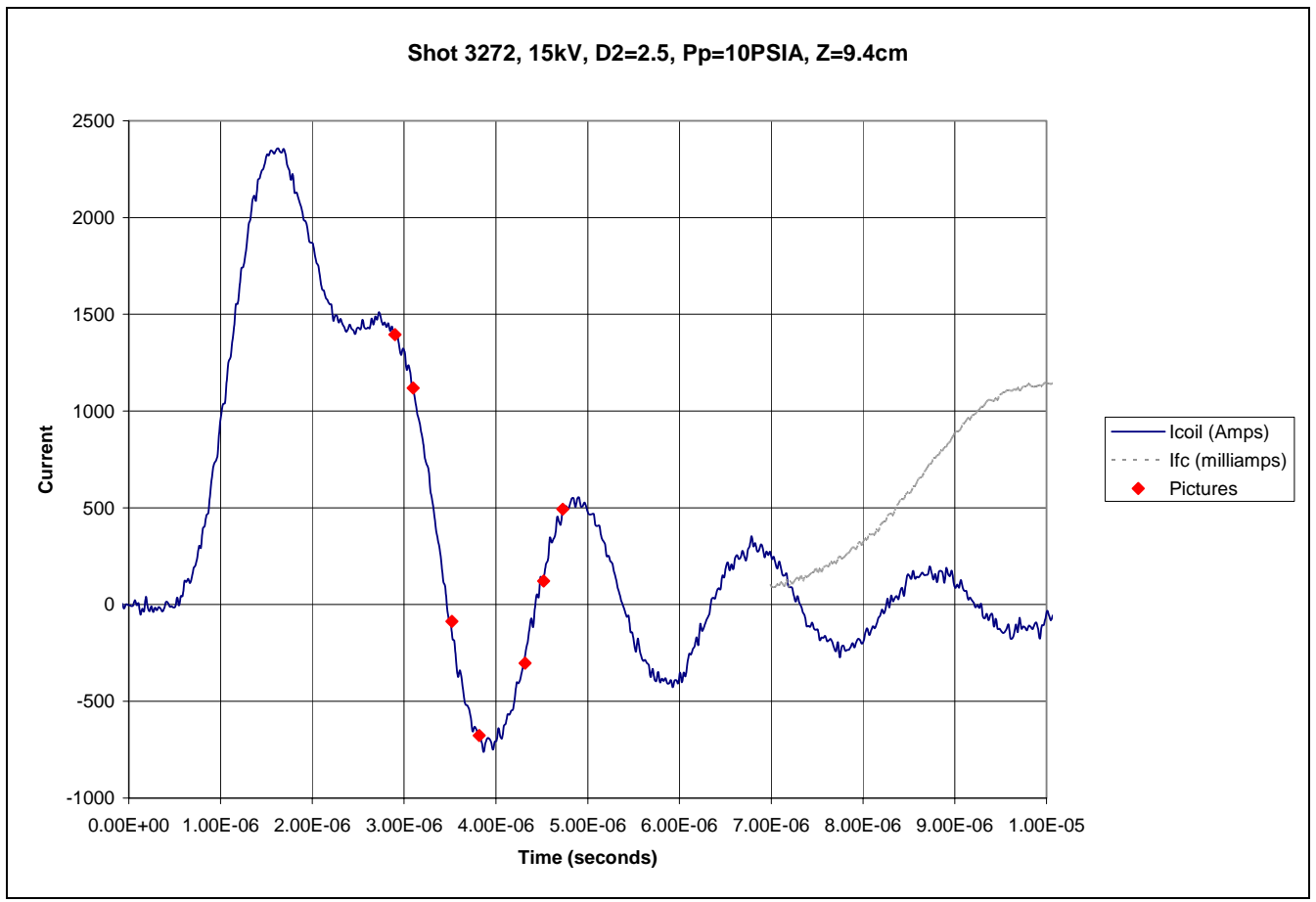

Figure 3.1.5: Timing of Front View Pictures Relative to Driving and Ion Currents 
A Dicam Pro ${ }^{\mathrm{TM}}$ Intensified CCD Camera was used to take images of the plasma from the front and side view. A time summary of these pictures can be seen in Figures 3.1.63.1.21. For the front view, two concentric lines have been added showing the position of the two driver coils. For the side view, lines have been added showing the edge of the nozzle and the plane of the driver coils. These pictures show the initial breakdown of the gas into plasma on the first zero-crossing. Then the pictures show the acceleration of a plug of plasma away from the plane of the driver coils. The pictures also show that the region directly in front of the driver coils continues to radiate on later cycles, which shows that plasma continues to exist there. However, while additional energy is radiated by the plasma in front of the plane of the driver coils, this plasma is magnetically confined to remain within that region. More information about magnetic fields will be presented in section 3.4.

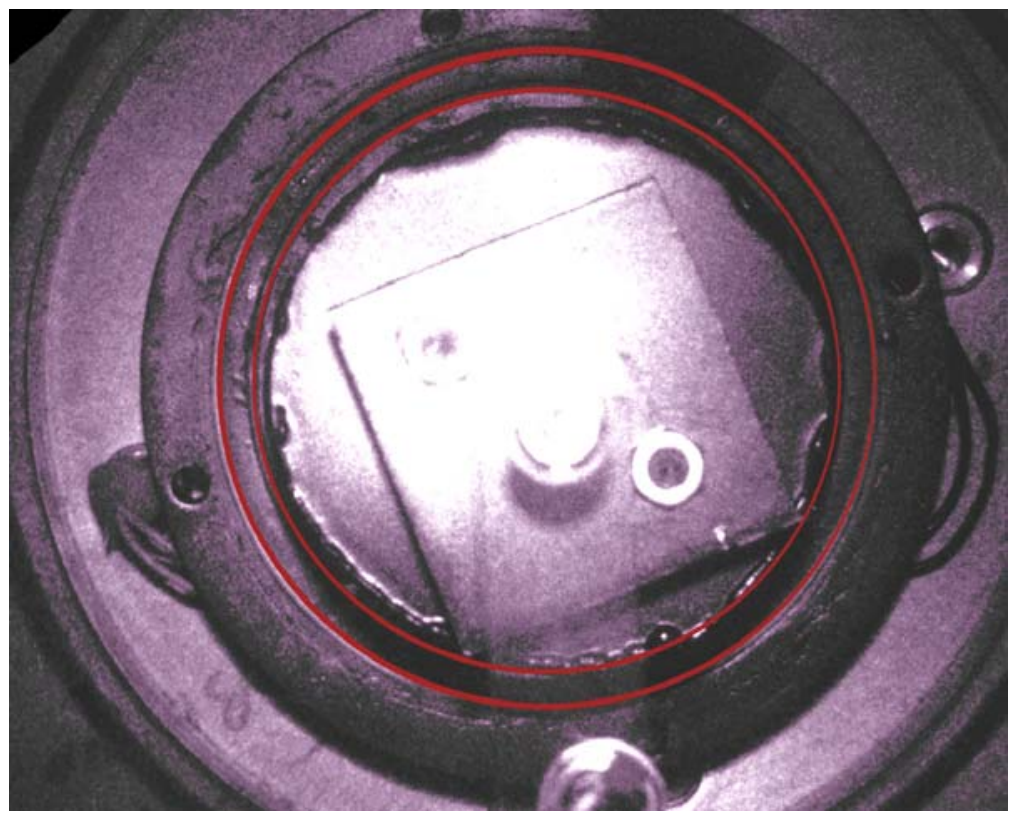

Picture 3.1.6: Front View Room Light 
Applied Pulsed Power, Inc.

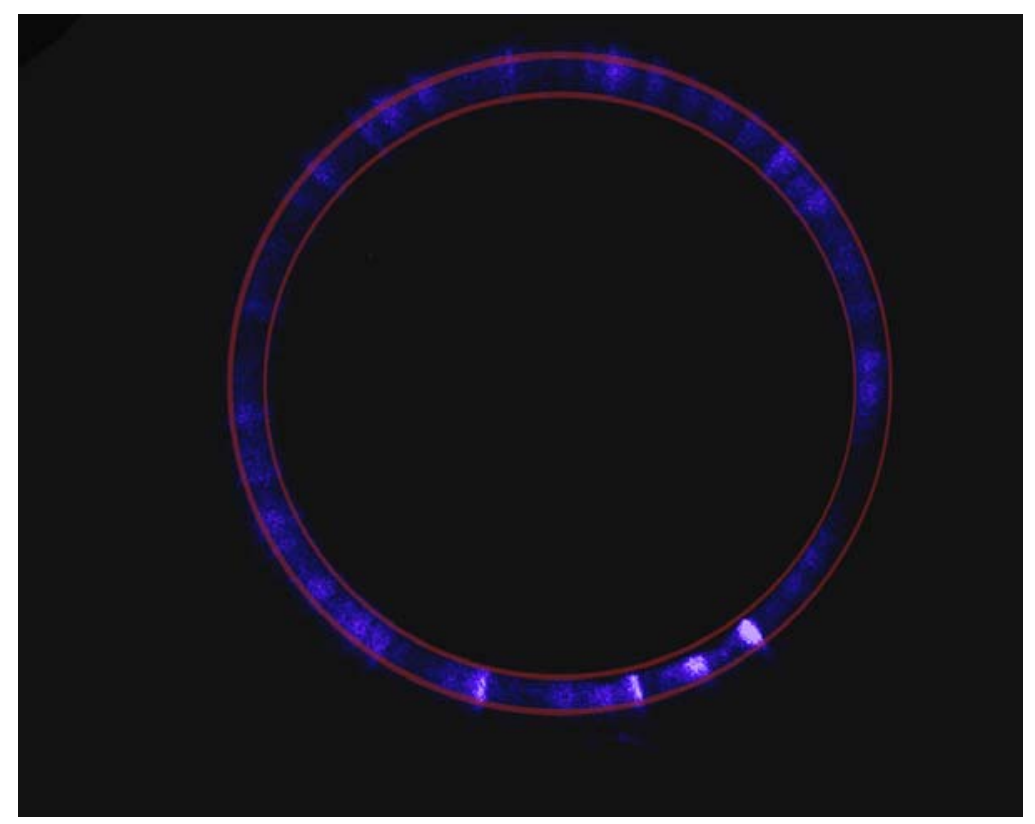

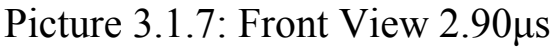

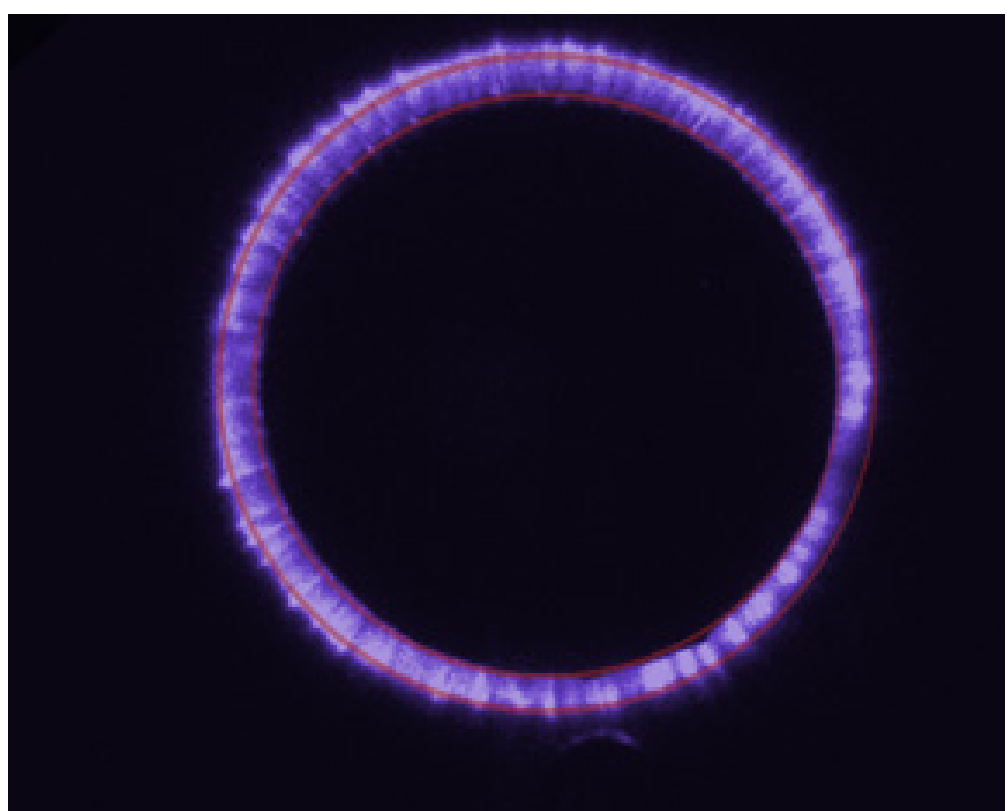

Picture 3.1.8: Front View 3.10 $\mu$ s 
Applied Pulsed Power, Inc.

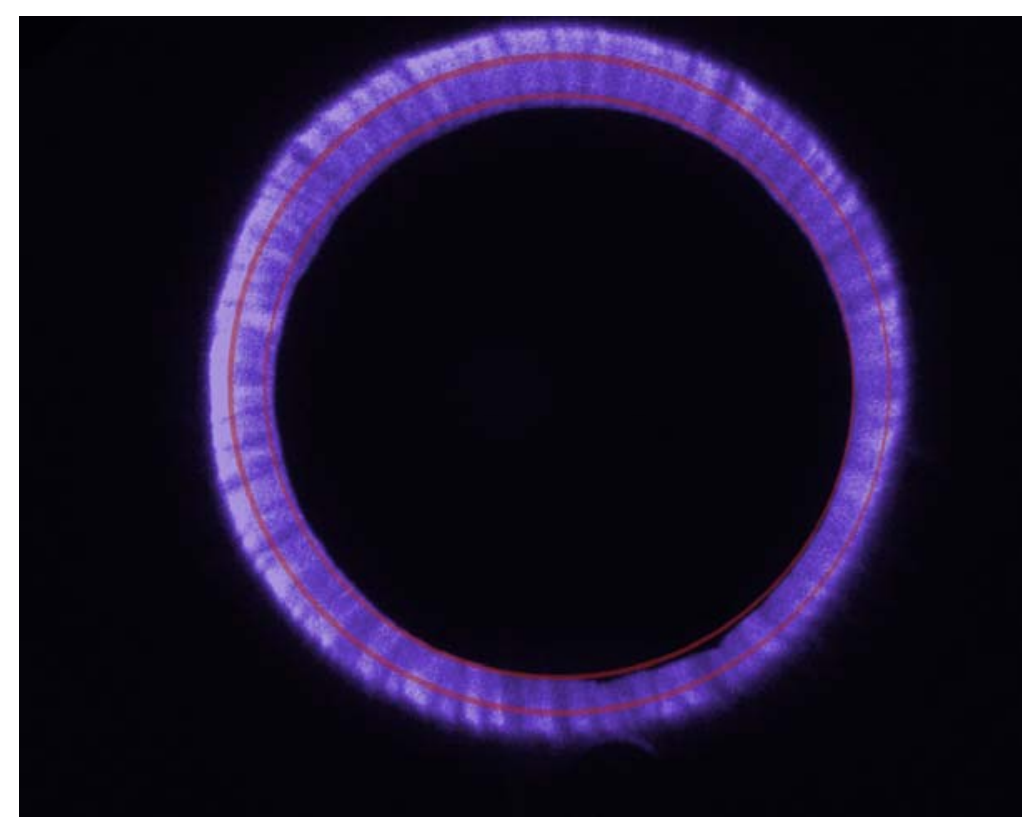

Picture 3.1.9: Front View 3.52 $\mu$ s

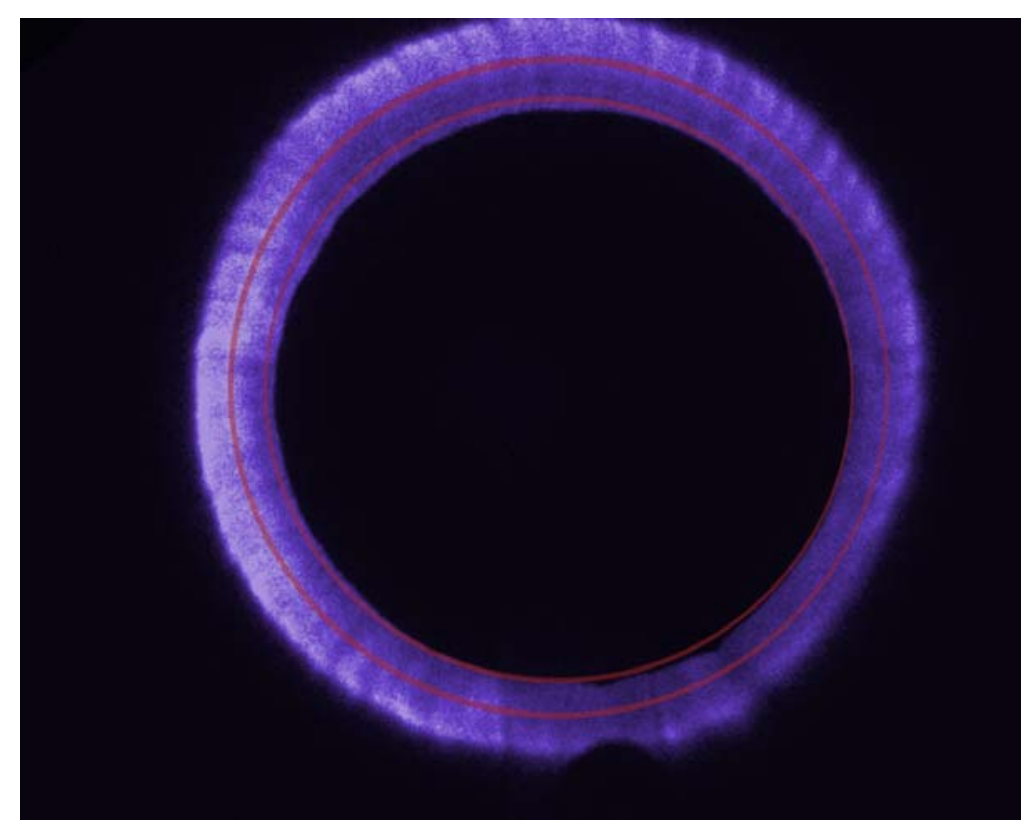

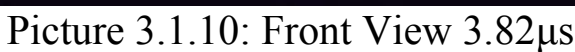


Applied Pulsed Power, Inc.

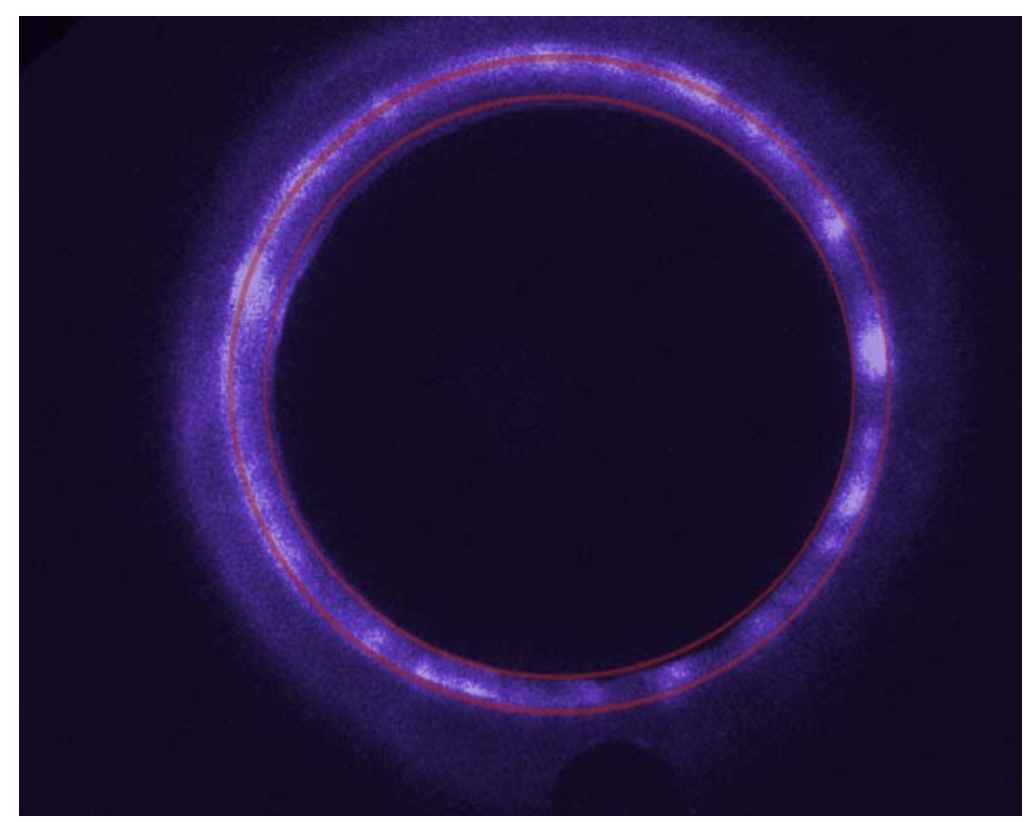

Picture 3.1.11: Front View 4.32 $\mu \mathrm{s}$

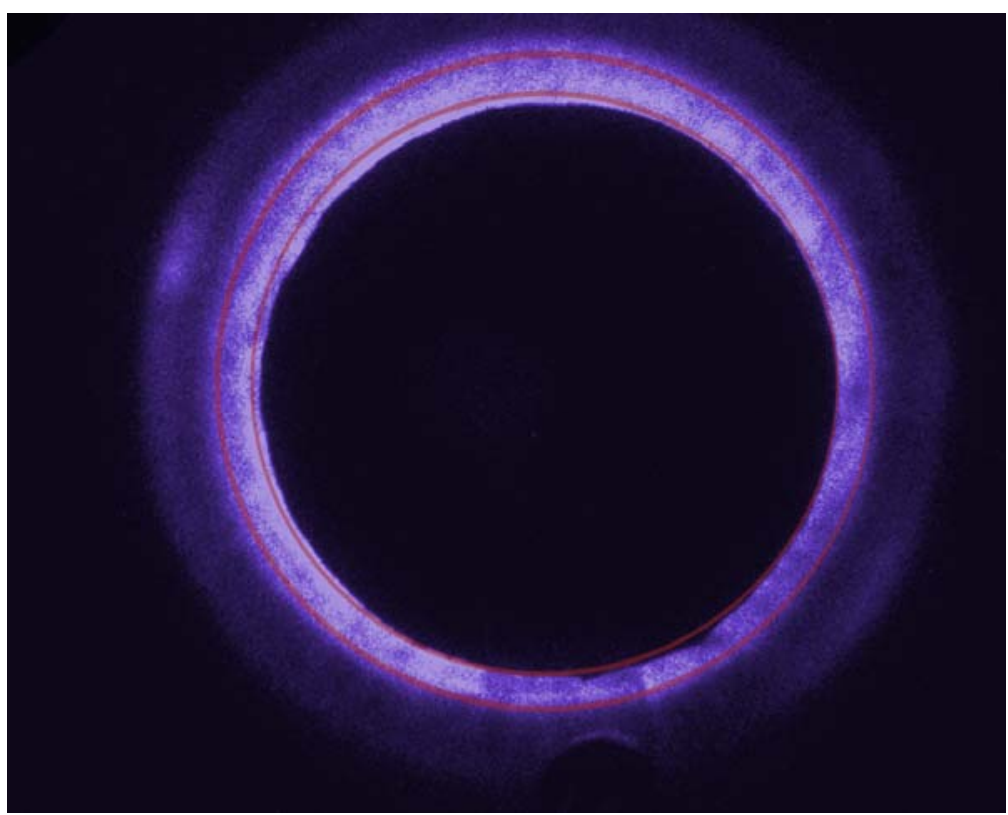

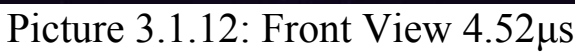


Applied Pulsed Power, Inc.

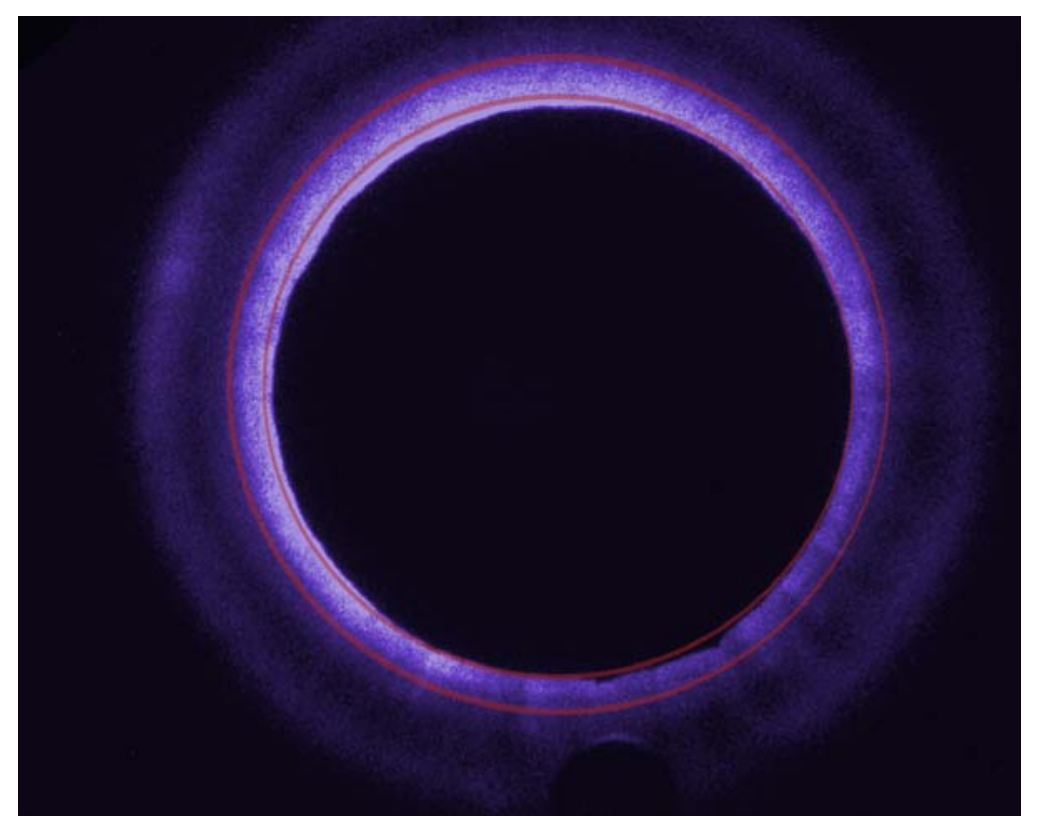

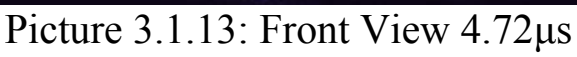

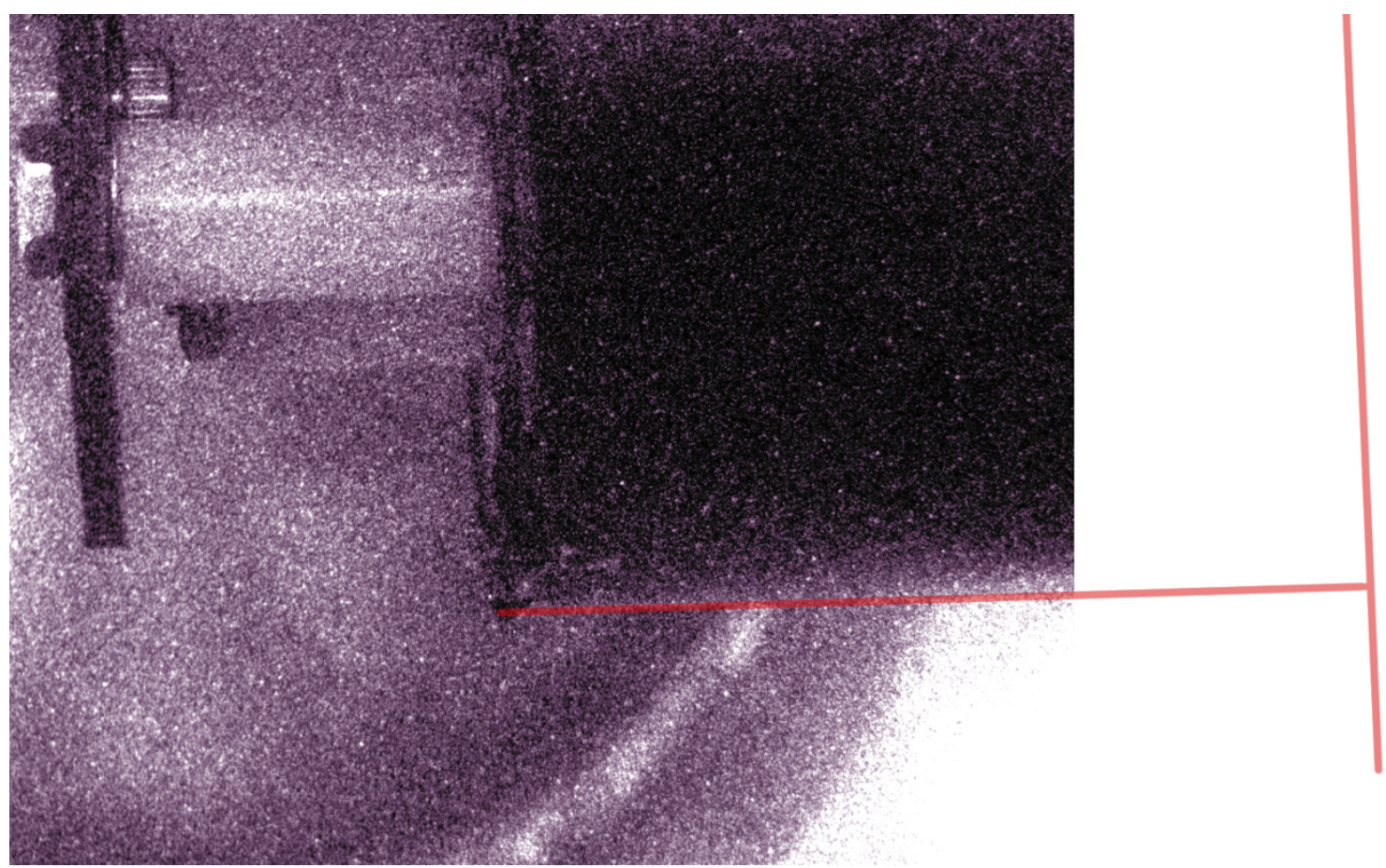

Picture 3.1.14: Side View Room Light 


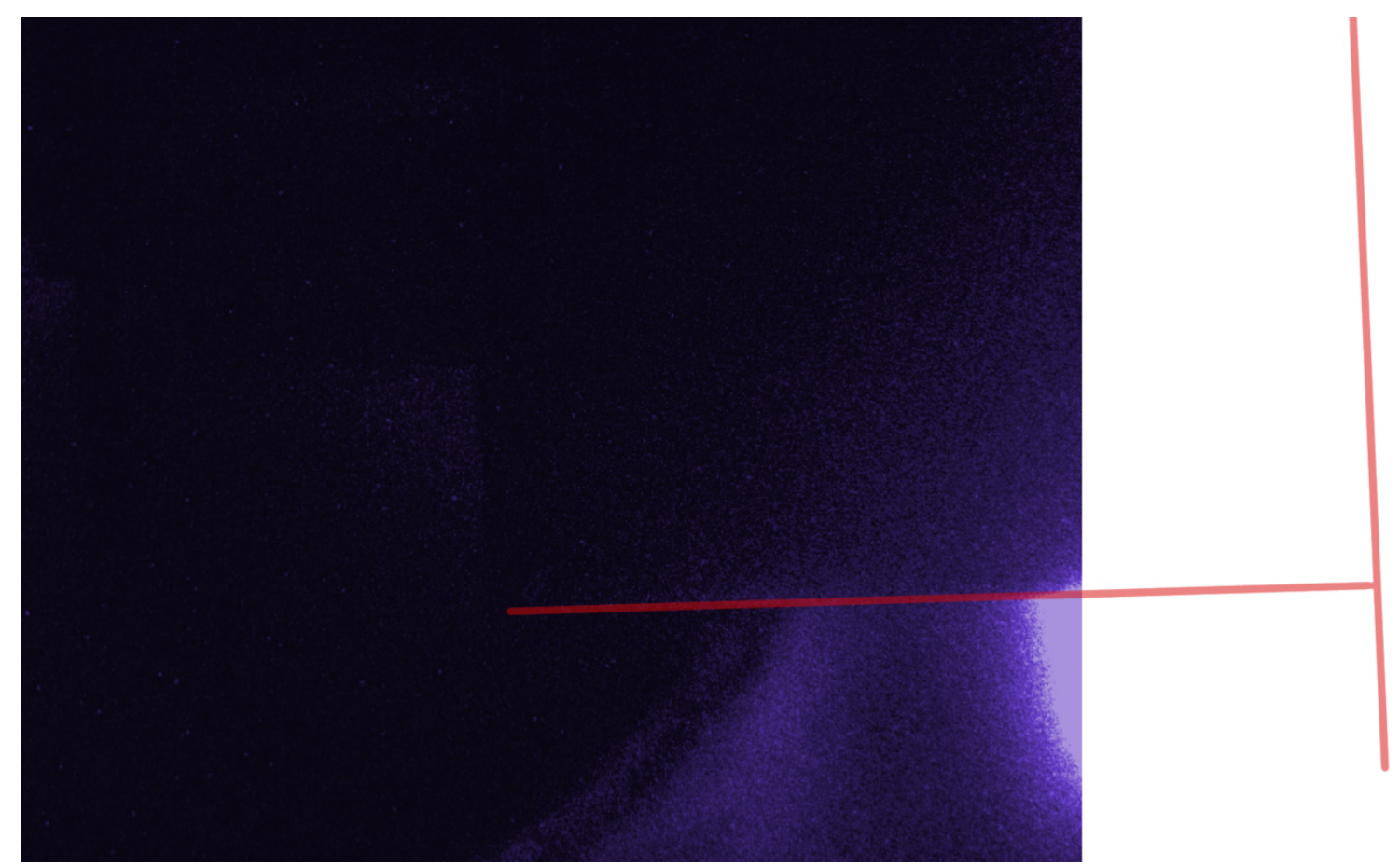

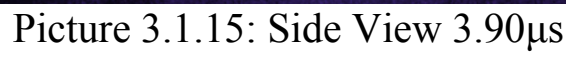

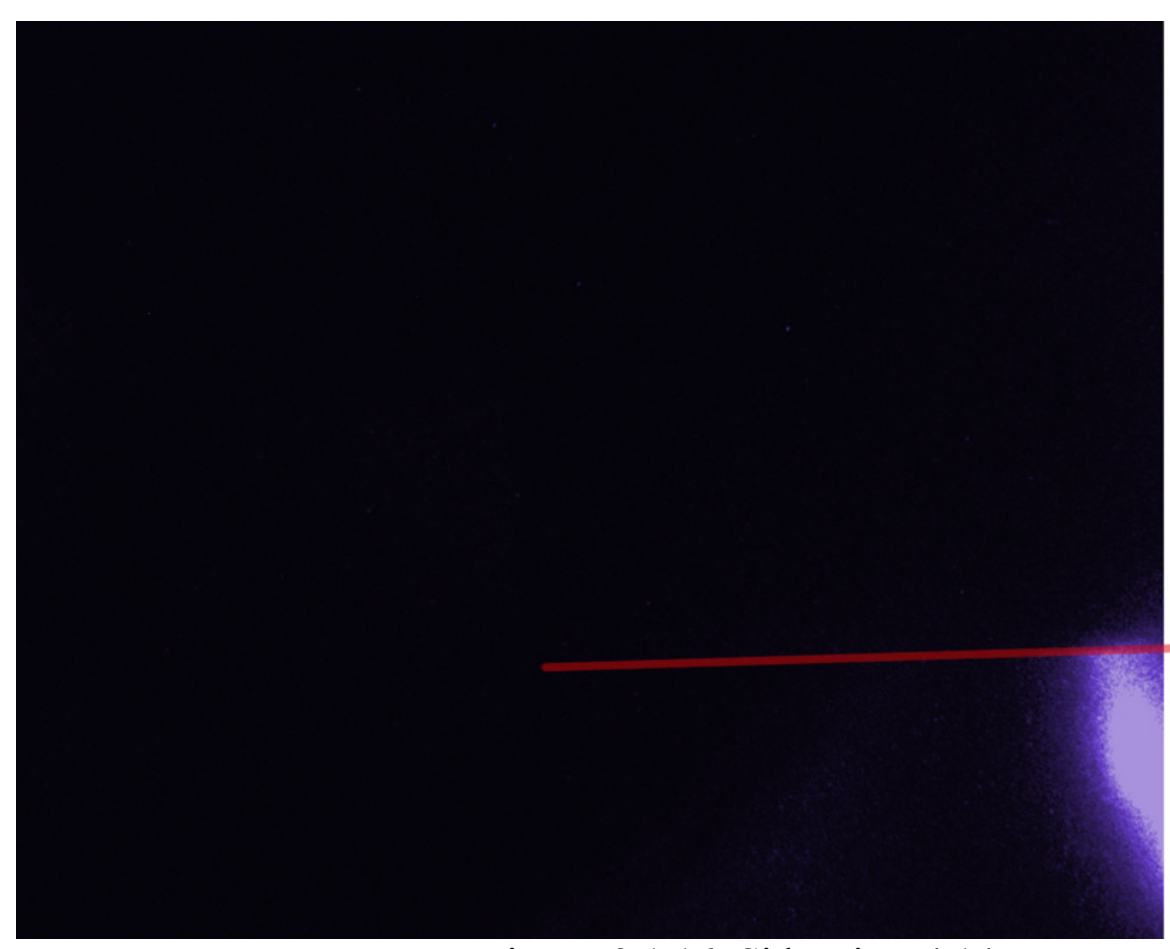

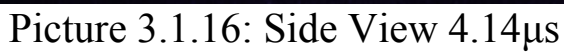




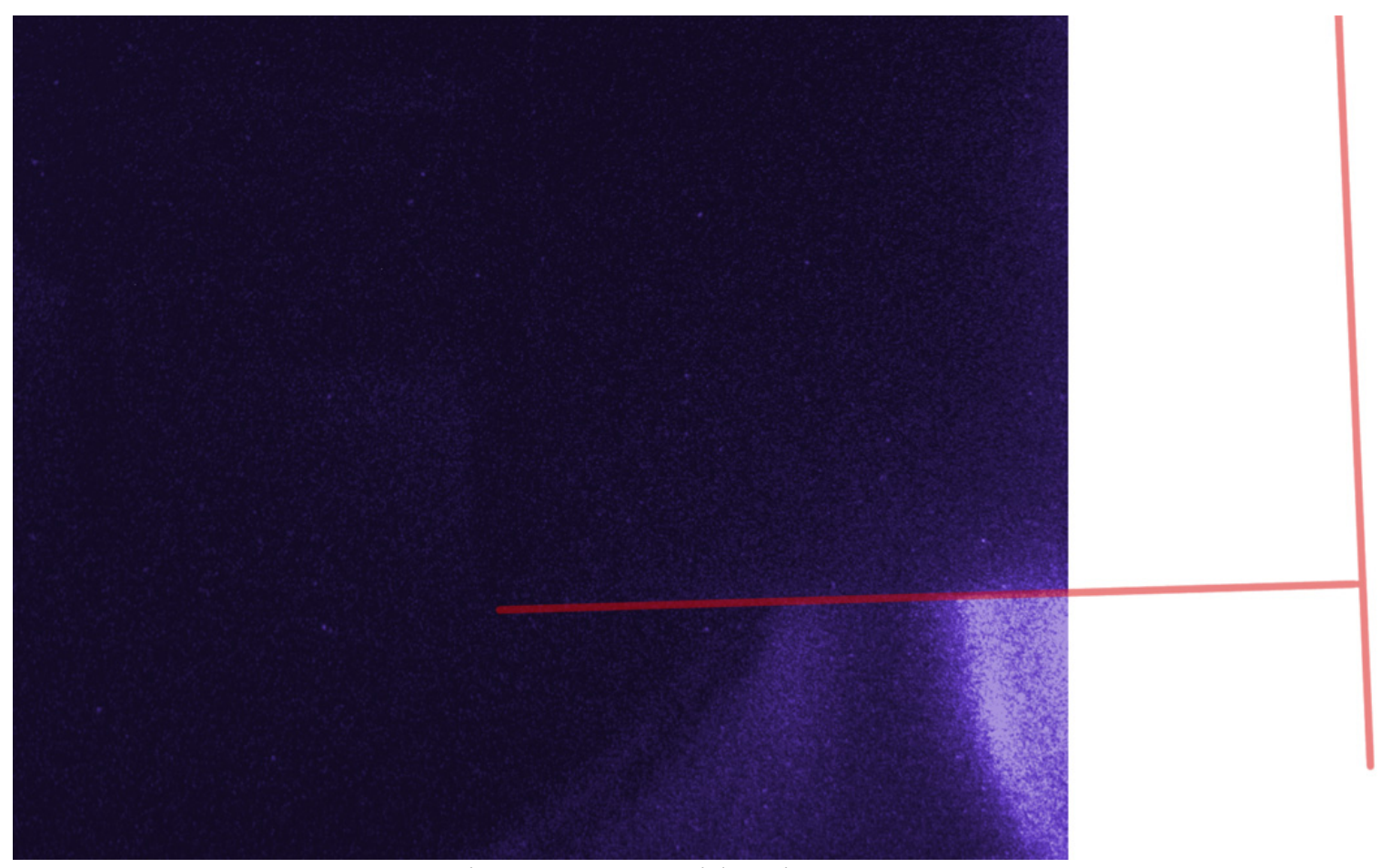

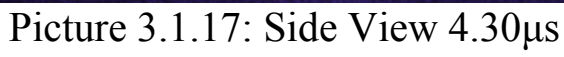

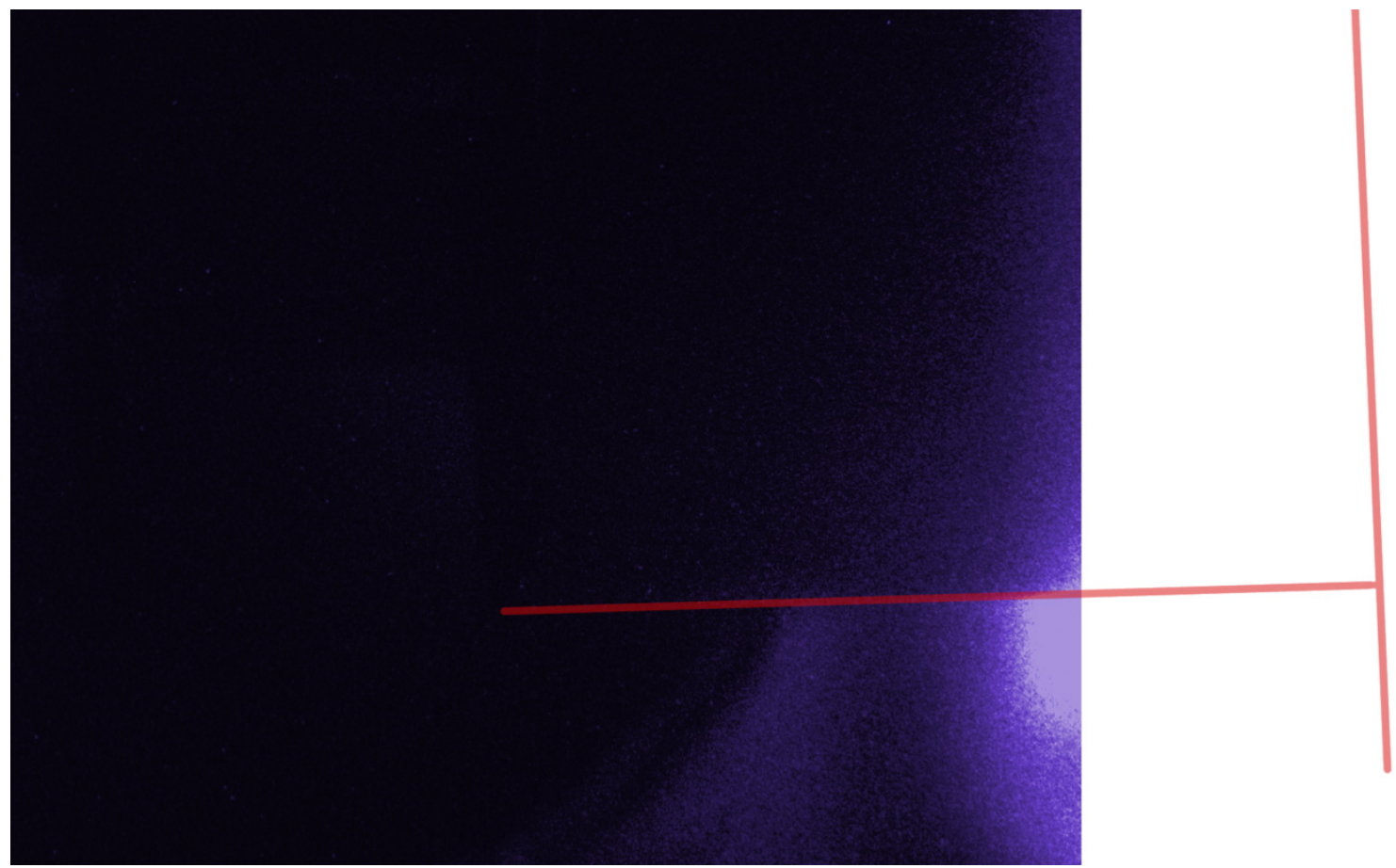

Picture 3.1.18: Side View $4.74 \mu \mathrm{s}$ 


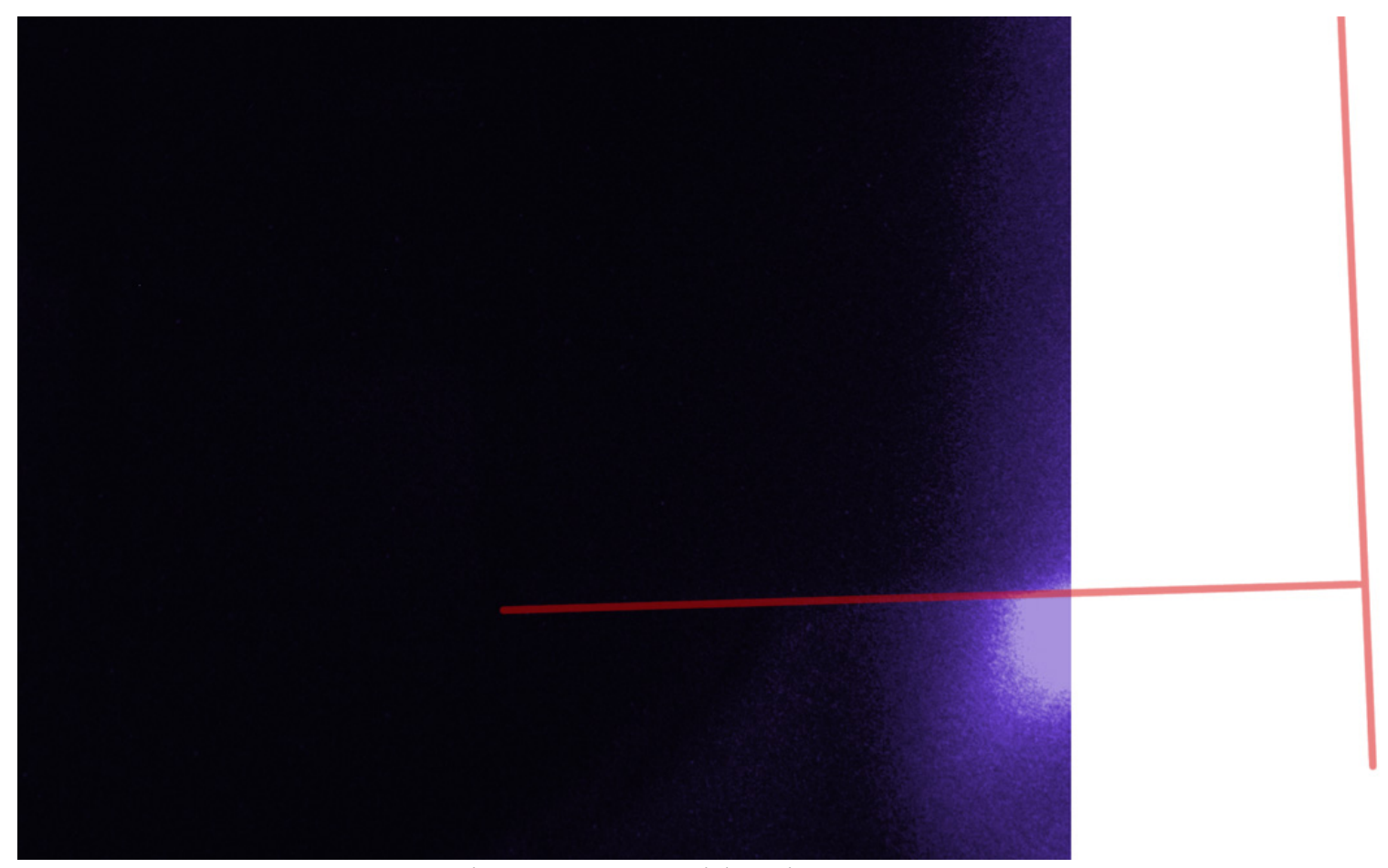

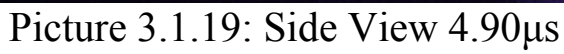

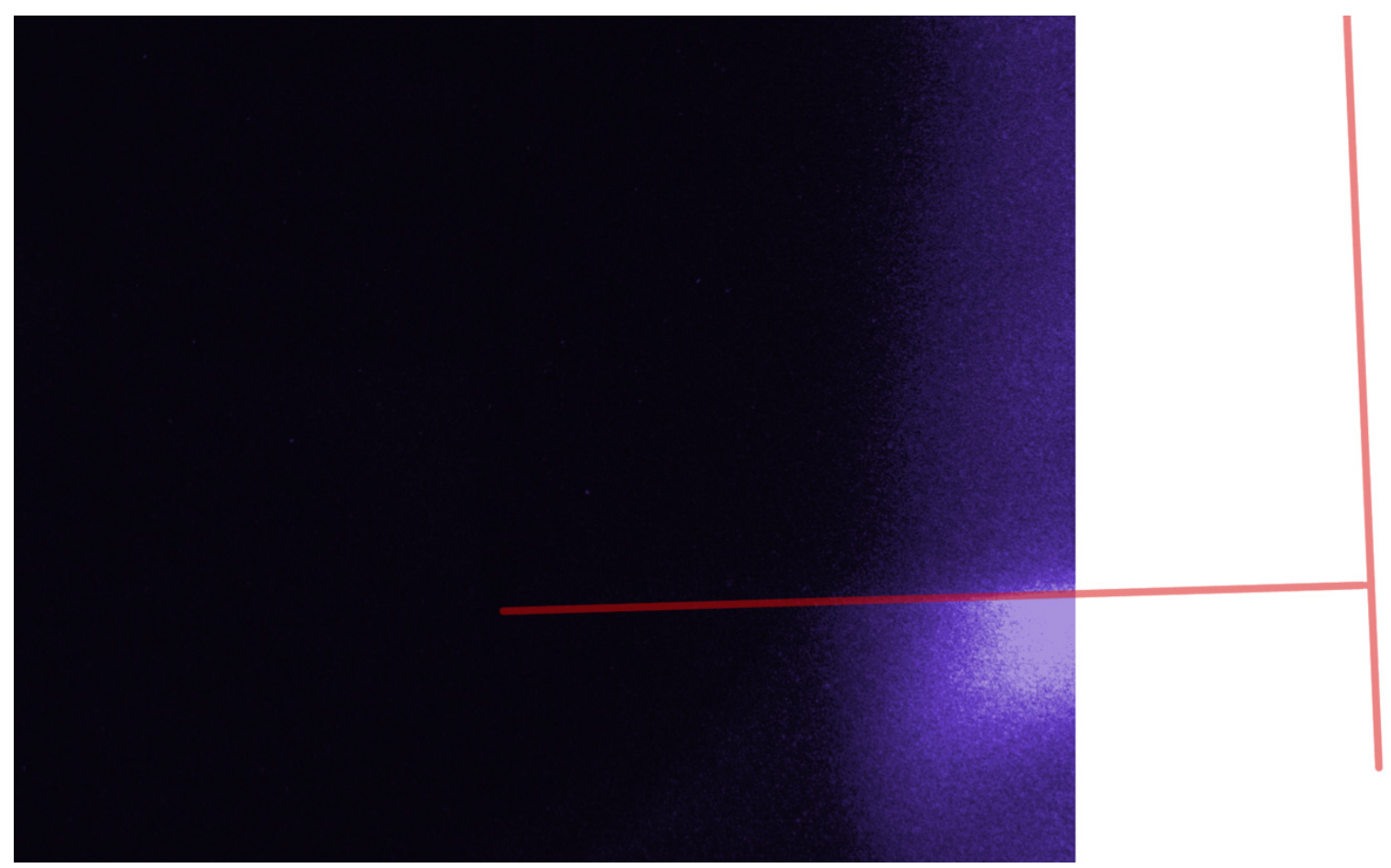

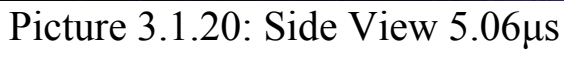




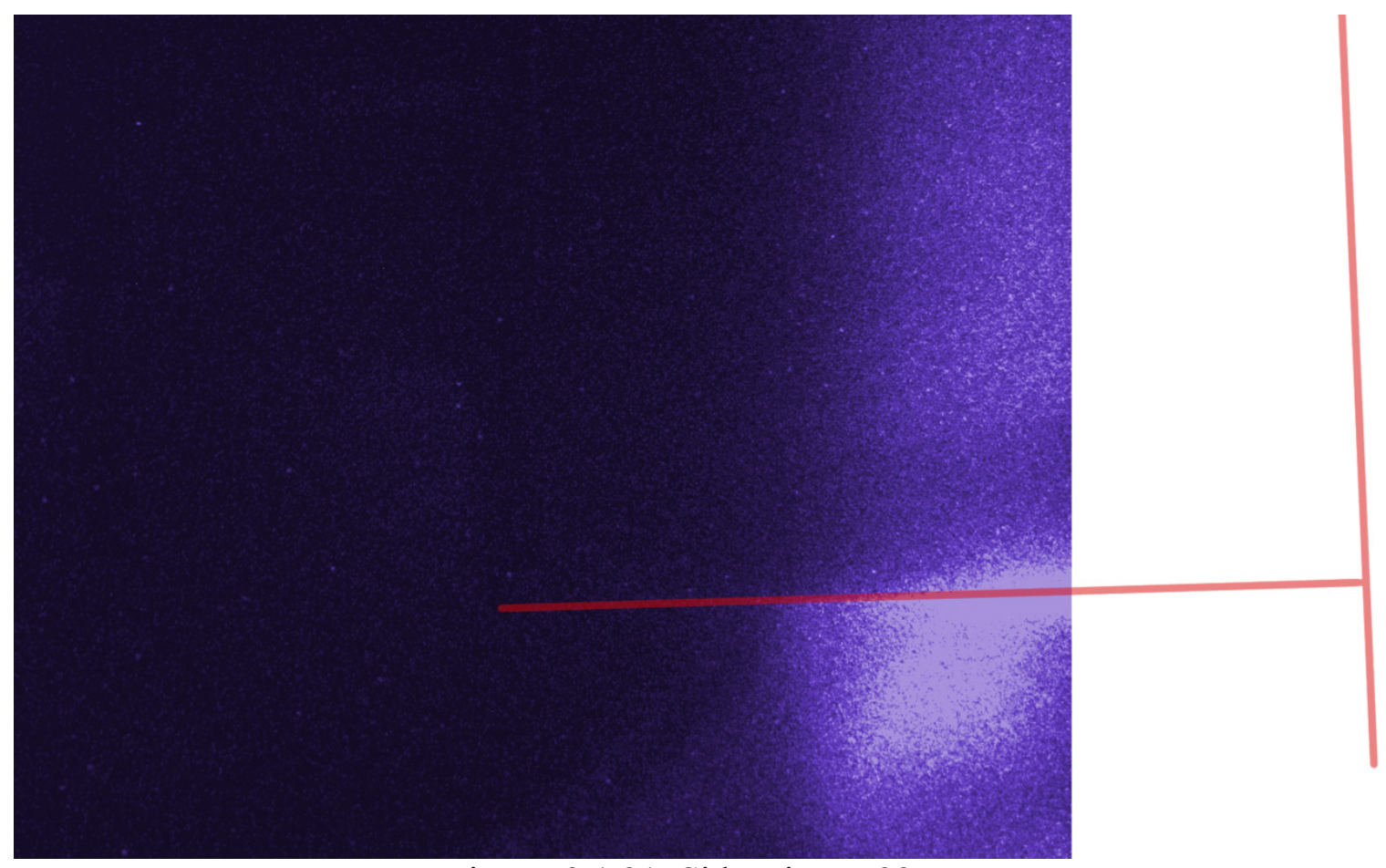

Picture 3.1.21: Side View 5.22 $\mathrm{s}$

\subsubsection{Phase II Source Induced Magnetic Fields}

The induced magnetic fields are the most critical part of the streaming plasma ion source. These fields determine the acceleration of the plasma. The acceleration and, to some extent, the divergence of the streaming plasma, results from the interactions of the alternating magnetic fields generated by the alternating current in the driver coils with the induced magnetic fields in the plasma. The magnetic fields in the plasma were therefore examined by $\mathrm{B}^{\circ}$ probes. These probes measure "Bdot", the time derivative of magnetic field, by measuring the voltage induced in a small wire loop by the time-varying field.

Figure 3.1.22 shows the normalized $\mathrm{B} \circ$ measurements with plasma while 3.1.23 shows the normalized $\mathrm{B}^{\circ}$ measurements without plasma. Figure 3.1.23 shows that without plasma, the measured normalized $\mathrm{B} \circ$ is similar at all three distances from the driver coils during the length in time displayed. When there is plasma, differences between the induced magnetic fields appear versus distance from the driver coils as shown in figure 3.1.22. After about $3 \mu \mathrm{s}$ on the time scale, the measured magnetic fields in the plasma at $0.5 \mathrm{~cm}$ diverge from those at $1.5 \mathrm{~cm}$ and $2.5 \mathrm{~cm}$. We know from the ICCD data presented in section 3.1.1, that breakdown of the neutral gas into plasma starts to occur at around that time. Further, the measured magnetic fields in the plasma at $1.5 \mathrm{~cm}$ diverge from those at $2.5 \mathrm{~cm}$ after about $4.8 \mu \mathrm{s}$ on the time scale. This time is associated with the separation of the accelerated plasma with a directed velocity away from the driver coils from the remaining plasma still trapped in front of the coils. 


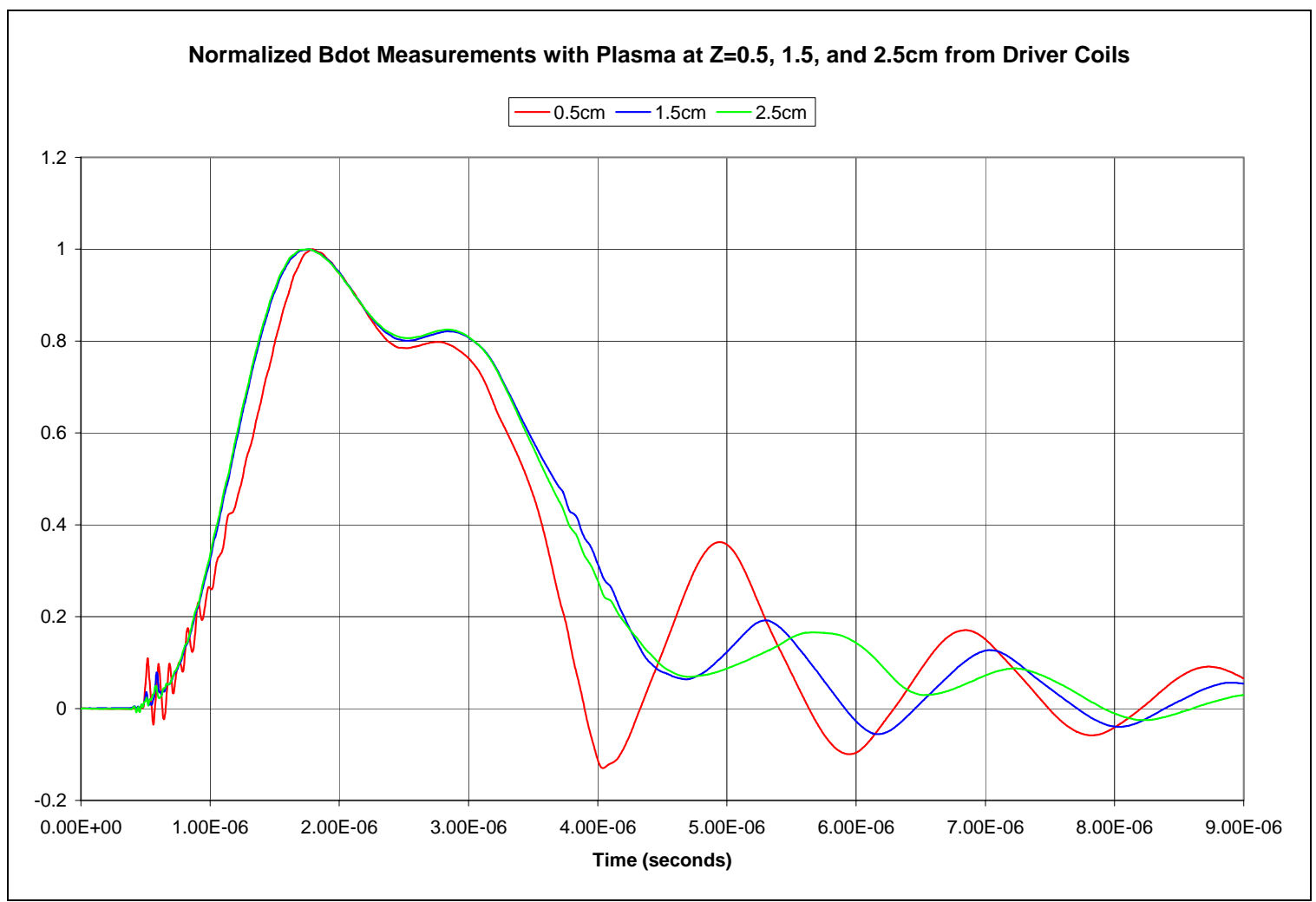

Figure 3.1.22: Normalized B॰ Measurements with Plasma

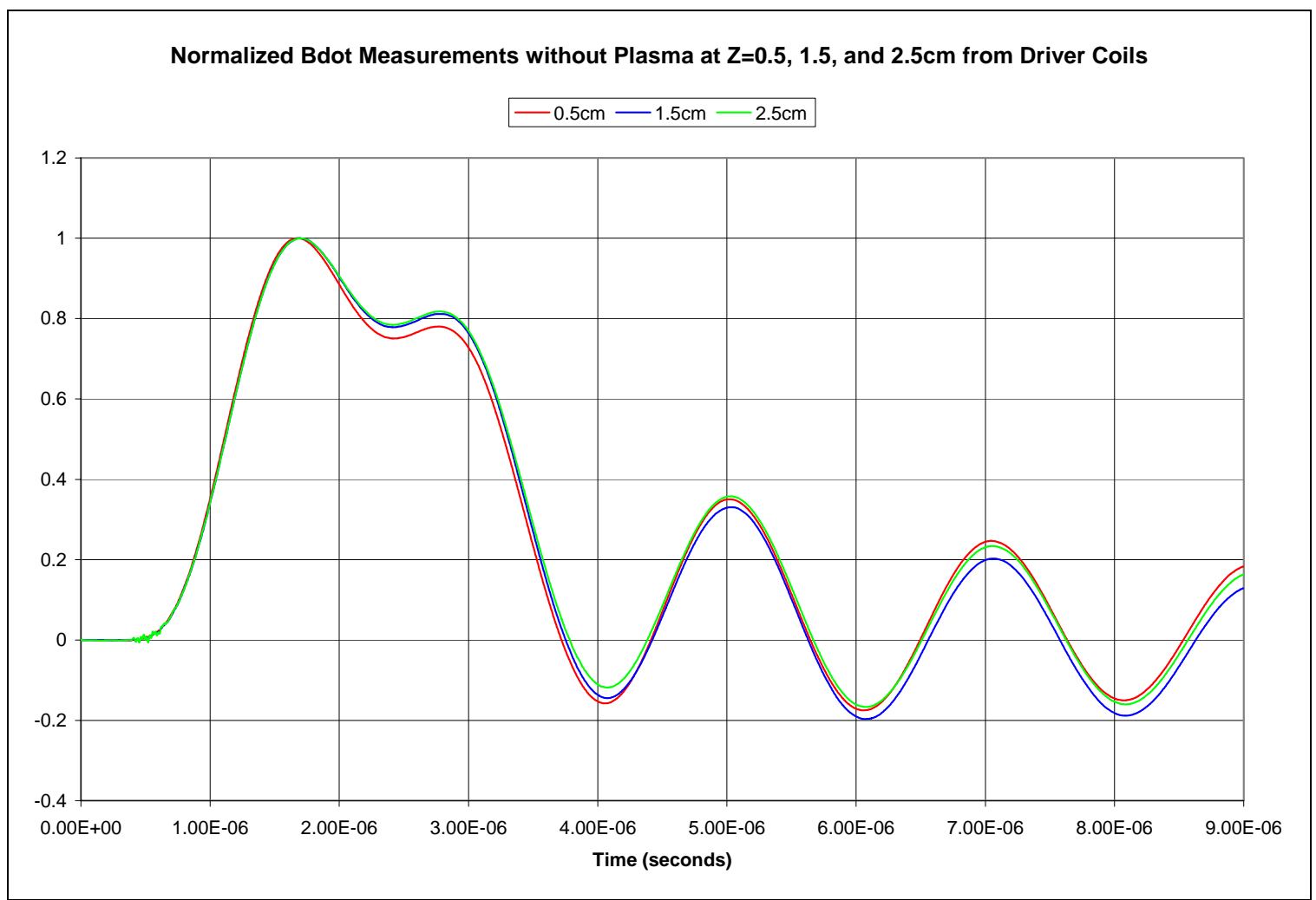

Figure 3.1.23: Normalized B ${ }^{\circ}$ Measurements without Plasma 


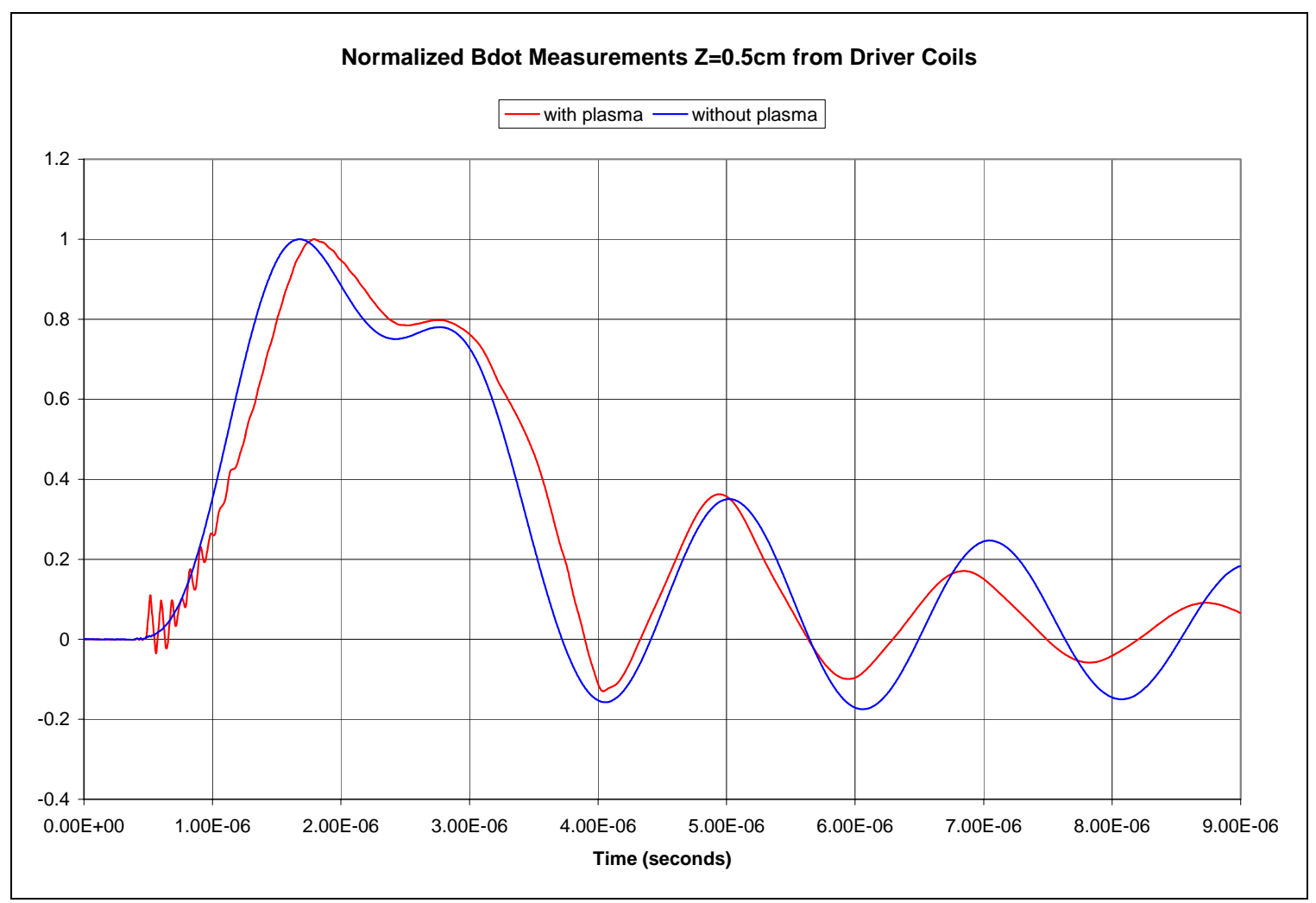

Figure 3.1.24: Normalized $B^{\circ}$ Measurements at $Z=0.5 \mathrm{~cm}$

Figure 3.1.24 further demonstrates the similarity of the measured magnetic fields at $0.5 \mathrm{~cm}$ from the driver coils with and without plasma. The difference in frequency seen is associated with the damping of the current in the driver coil caused by the plasma. Figure 3.1.25 shows the proportional decrease in absolute measure magnetic field with increased distance from the driver coils, as would be expected. This data, when taken with the ICCD images, demonstrate that the plasma separates into two regions, one trapped in front of the driver coils and another which drifts away from the area in front of the driver coils. The plasma trapped in front of the driver coils acts as a flux excluder, preventing the induced magnetic fields caused by the alternating currents in the driver coils from affecting the plasma drifting away from the area in front of the driver coils. 


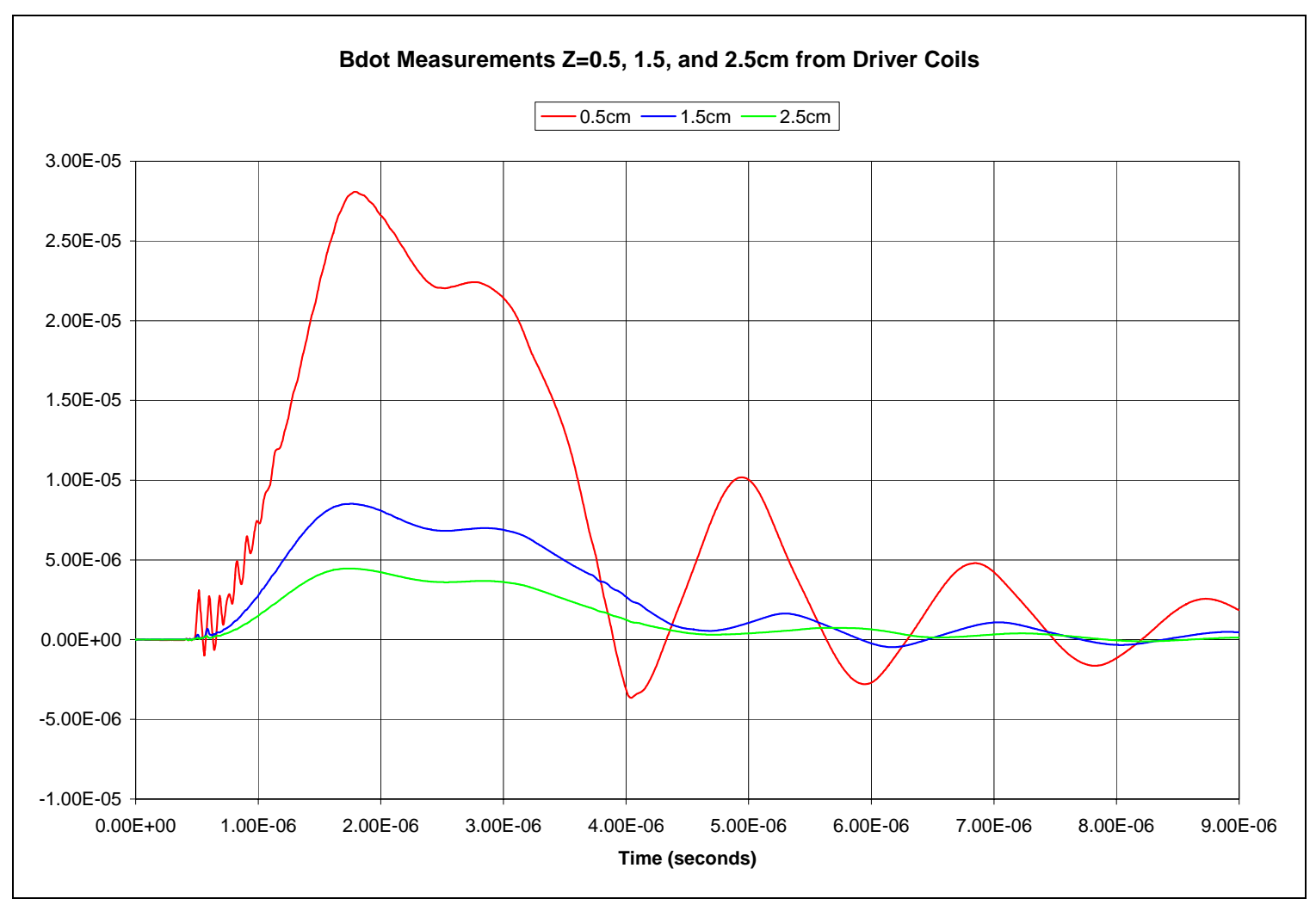

Figure 3.1.25: $\mathrm{B} \circ$ Measurements

Further analysis of the magnetic fields using different delays between the gas injection and the driver coil current clarified the correlation between the observed plasma motions seen in the ICCD images with the times that the normalized $\mathrm{B} \circ$ measurements varied at the different distances from the driver coils. For example, at longer delays between the driver coil current and the gas injection, which results in a higher background pressure of the neutral Argon, the plasma separates at a later time than the results shown above. The opposite is also true, that shorter delays result in earlier separation. This can be seen in figure 3.1.26. The correlation demonstrates that at longer delays, the streaming plasma has a slower velocity and originates from later in time than with shorter delays. 


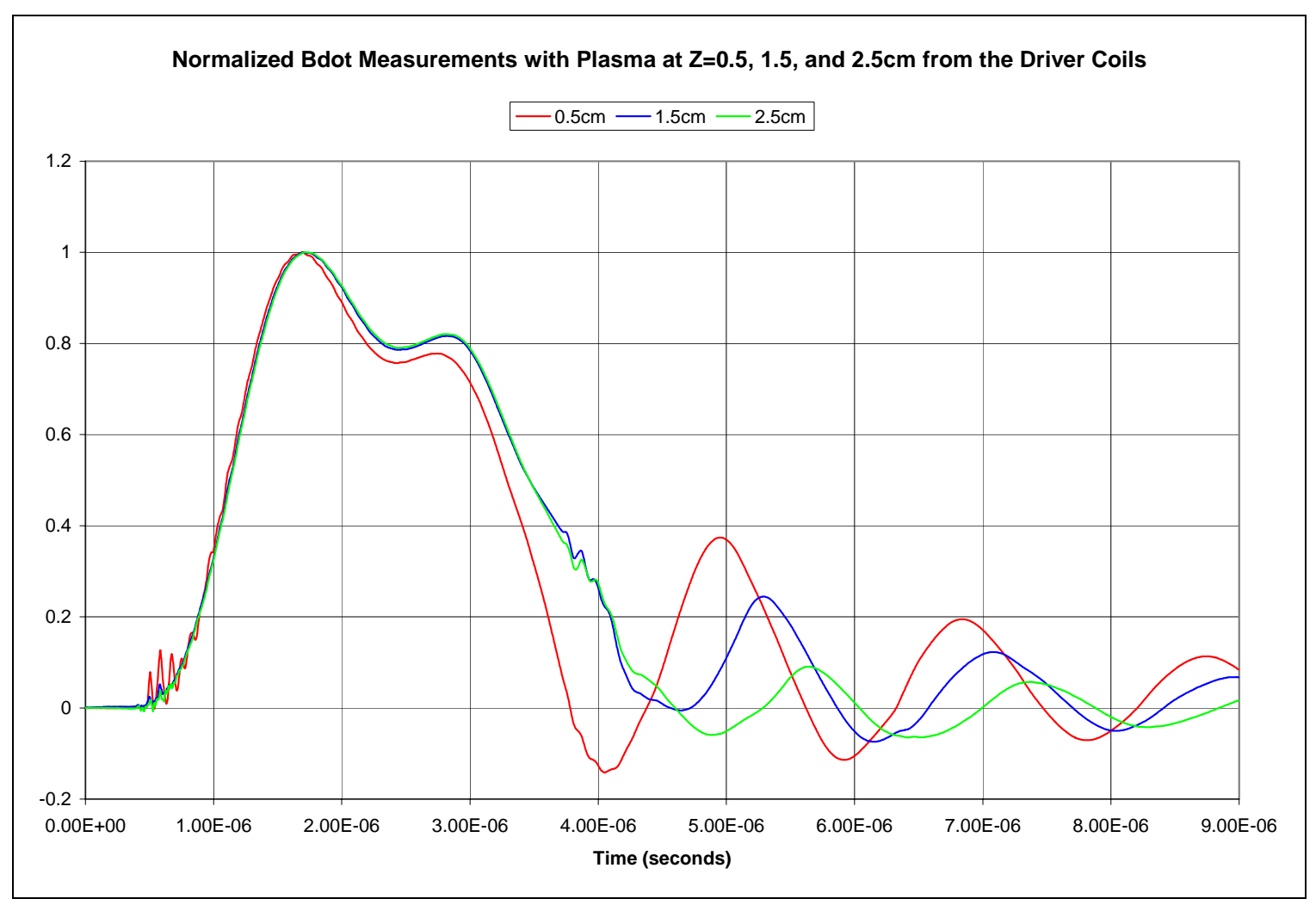

Figure 3.1.26: B॰ Measurements at a Shorter Delay

\subsubsection{Development of Operational Settings for the Phase II Source}

With the mechanical and electrical design of the Phase II Source complete, the next step was to determine the operational settings required to optimize the source performance. To allow for control over the source, the following conditions could be adjusted on a shot-toshot basis:

- The plenum pressure of the gas inlet valve.

- The charge voltage of the pulsed power controlling the opening of the gas inlet valve.

- The charge voltage of the driver pulsed power.

- The delay between the discharge of the pulsed power controlling the opening of the gas inlet valve and the discharge of the driver pulsed power.

The parameter space for these settings was examined to determine appropriate conditions within certain restrictions. For example, it was decided that the plenum pressure should be kept above atmospheric pressure to better maintain the purity of the Argon gas supply. Two settings were examined, 1450 torr and 1150 torr. To achieve the best reproducibility, the higher plenum pressure, 1450 torr, was chosen. The charge voltage of the pulsed power controlling the opening of the gas inlet valve was determined to have little impact on the resultant streaming plasma, if the delay timing was altered to adjust to the new time of arrival of the gas injected into the source. A voltage setting of 575 Volts was chosen. 
The minimum setting of the charge voltage of the driver pulsed power that resulted in plasma formation was around $11 \mathrm{kV}$. The ion current flux into a downstream Farady cup increased as the driver pulsed power charge voltage increased. The incremental increase was less significant around $15 \mathrm{kV}$, which became the chosen operating voltage. This level proved to be fairly reproducible when examined with the ICCD, $\mathrm{B}^{\circ}$, and Faraday cup measurements. Figure 3.1.27 shows four independent data sets recording the ion flux in a Faraday cup downstream from the source. This shows the typical approximate $\pm 5 \%$ variation in the ion flux.

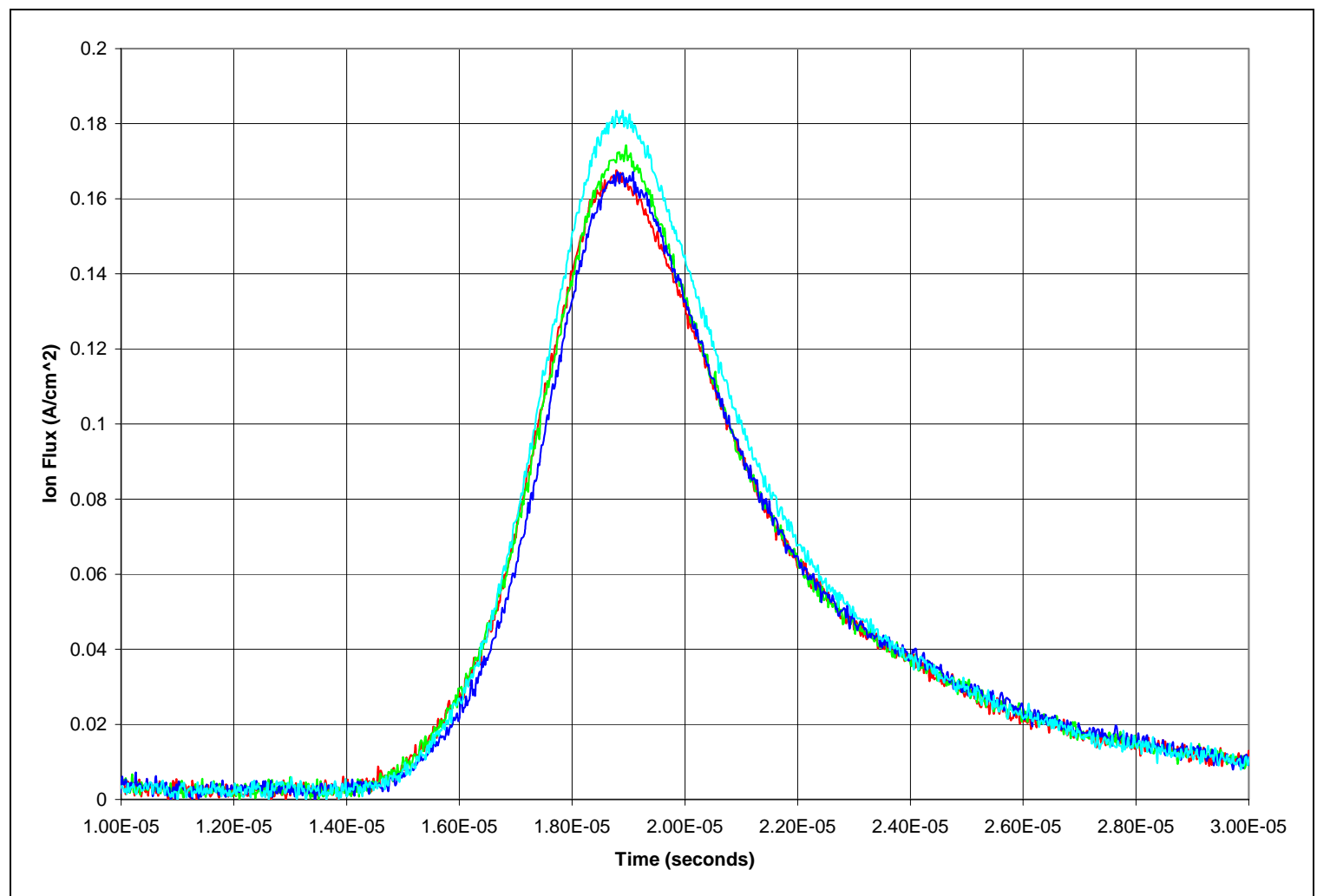

Figure 3.1.27: Ion Flux for 4 Independent Pulses

The most important parameter was the delay timing between the gas injection and the driver. It has been determined that the desired gas breakdown requires a certain minimum gas density, necessitating at least a corresponding minimum delay between the gas injection and the driver. The minimum delay for the Phase II source is found to be around $240 \mu \mathrm{s}$. As the delay increases from this timing, the gas density will increase, providing more neutral gas, which will allow for denser plasma. Eventually the falling edge of the gas density profile in front of the driver coils would generally set a maximum useable delay, but another factor contributes which requires a shorter maximum delay. This factor is the downstream neutral gas density, which increases with time. Downstream neutral gas density results in more collisions of the ions with neutral gas, thus reducing the average velocity of the streaming plasma. The peak ion flux is then reduced as well, which increases the temporal pulse width of the streaming plasma, and the divergence of the streaming plasma. So while increased gas density in front of the driver coils results in a higher plasma density and therefore a larger ion flux in the 
streaming plasma, the higher downstream neutral gas density results in a lower ion flux extracted downstream. Figure 3.1.28 shows the effect of delay timing on the ion flux in a Faraday cup downstream from the source.

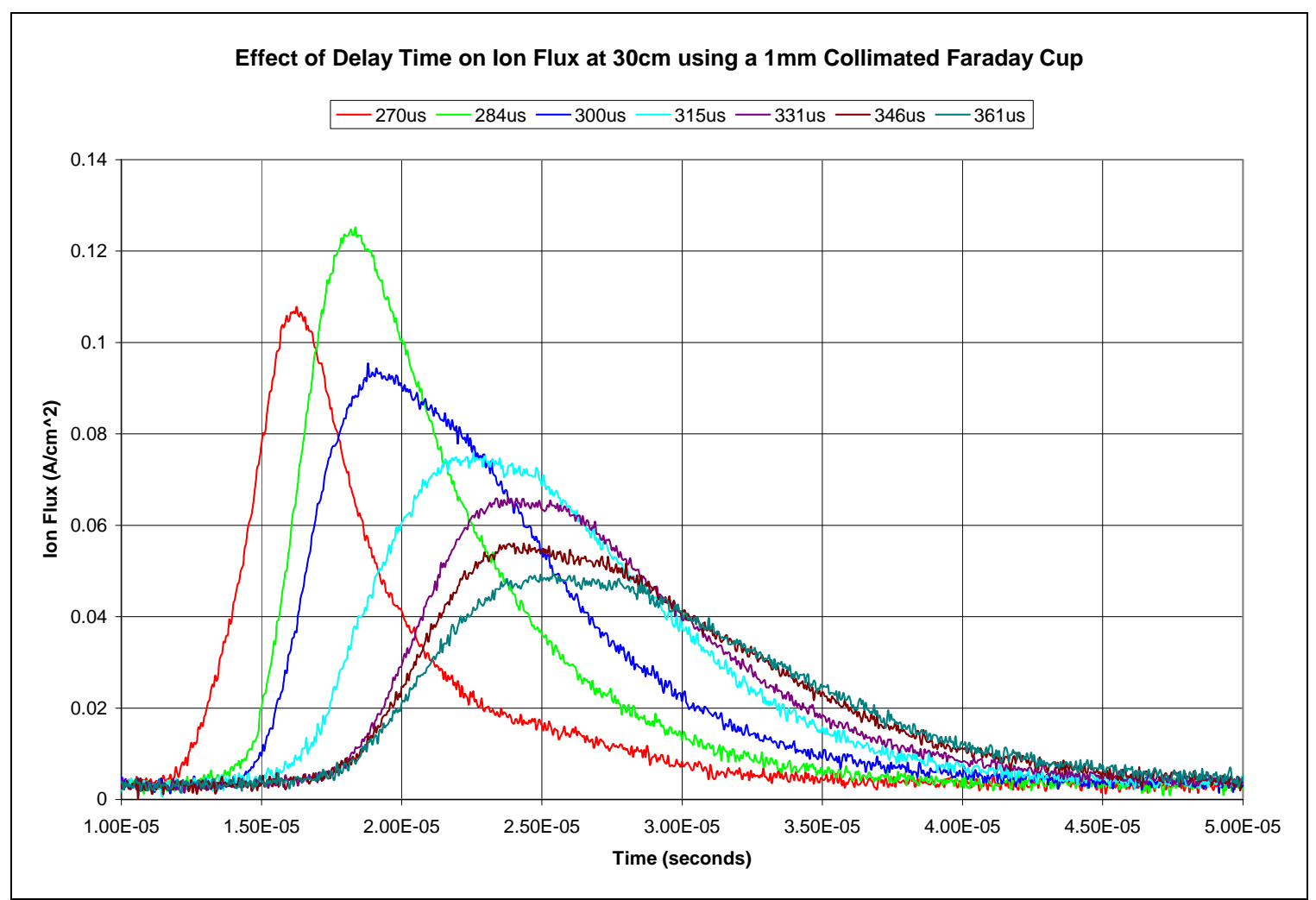

Figure 3.1.28: Variation in Ion Flux with Delay Timing

\subsection{Description of Modulator}

A new feature invented during the development of the source was a method to alter the neutral ion beam current magnitude downstream from the source. This method was called the modulator. Figure 3.1.2 shows a side view of the modulator and its position relative to the source. Figure 3.2.1 shows a front view of the modulator. The varying current on a pair of concentric and coplanar coils creates magnetic and electric forces on the streaming plasma. These forces can be used to inhibit or even prevent the streaming plasma current that would otherwise flow through the area located between the two coils, without otherwise altering the streaming plasma. 


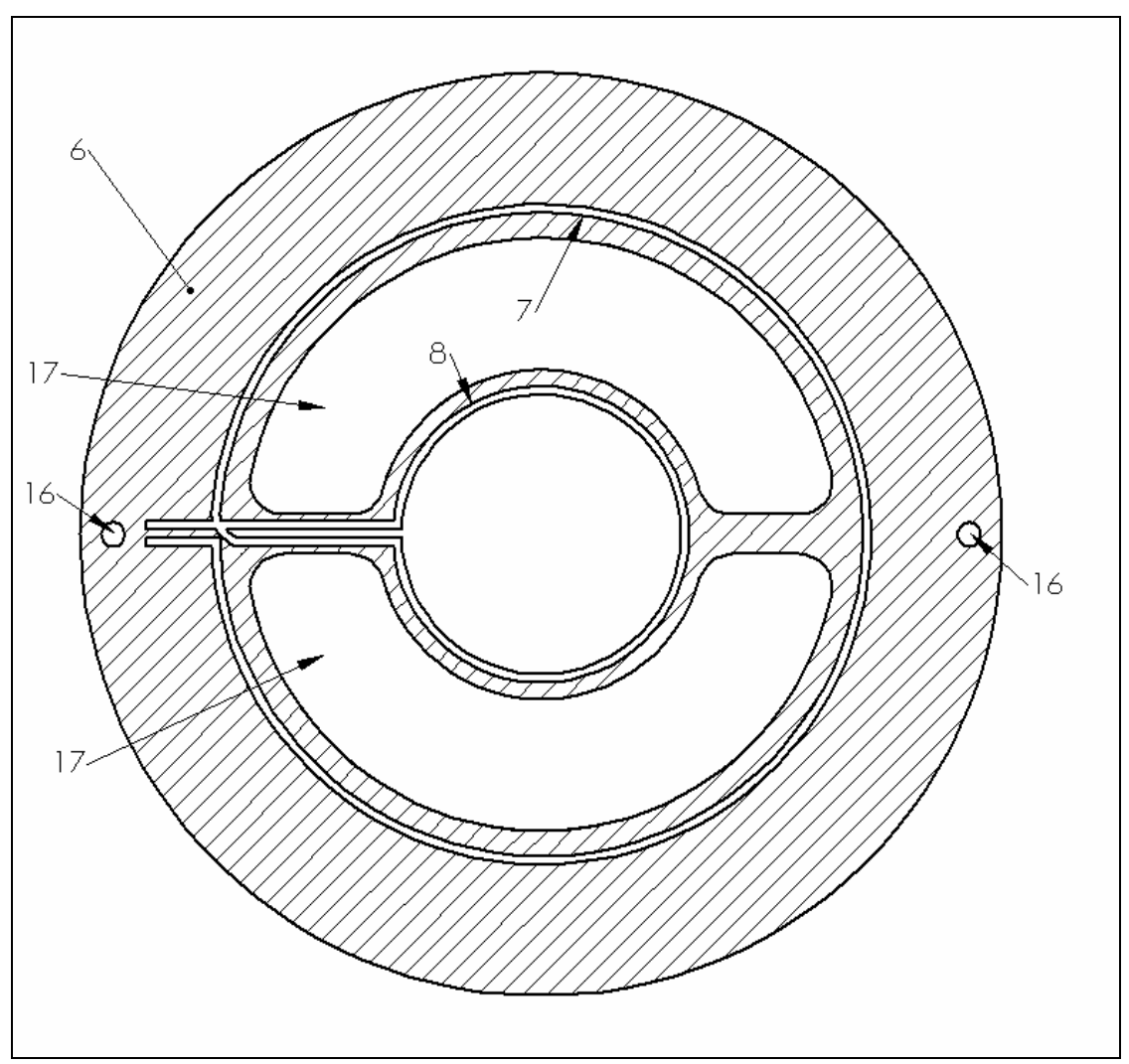

Figure 3.2.1: Modulator

Figure 3.2.2 shows the results of using different current levels on the modulator (different charge voltages on the modulator pulsed power supply). As shown, by using a modulator timing that coincides with the peak of the ion beam current, the modulator can flatten the top of the normally strongly peaked ion current. It can also be noticed from this figure that the peak current density without the modulator is around $2 \mathrm{~A} / \mathrm{cm}^{2}$ at $10.5 \mathrm{~cm}$ from the source. The modulated peak current density is still $300-500 \mathrm{~mA} / \mathrm{cm}^{2}$, well above the desired $100 \mathrm{~mA} / \mathrm{cm}^{2}$ current density. Another use of the modulator would be to create a faster rising pulse. Figure 3.2.3 shows the use of the modulator at different timings, and the associated effect. These data provide proof-of-principle evidence that a suitably shaped modulator waveform could shape the ion current as desired, over a fairly wide range of temporal shapes. 
Applied Pulsed Power, Inc.

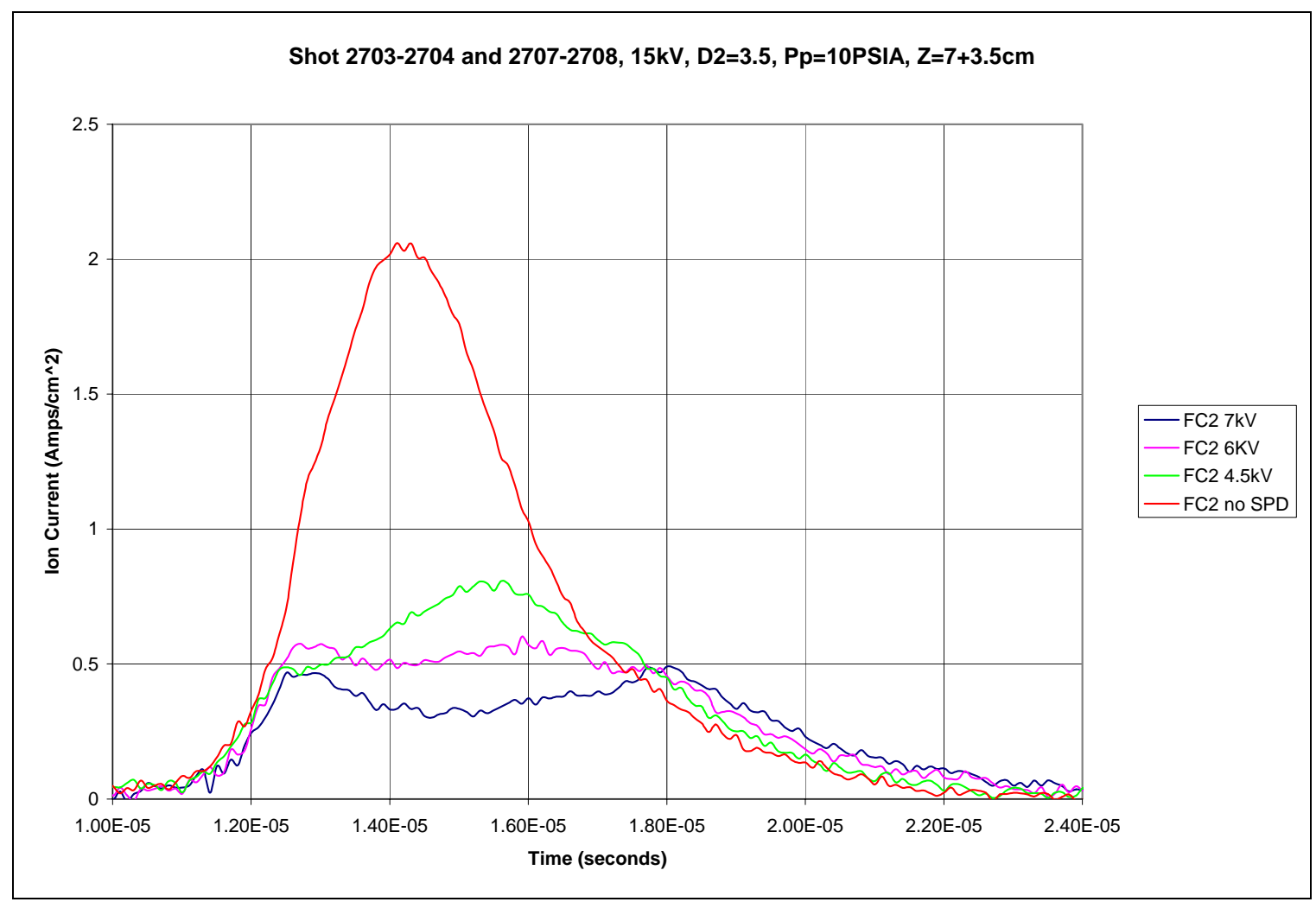

Figure 3.2.2: Modulation of the Streaming Plasma

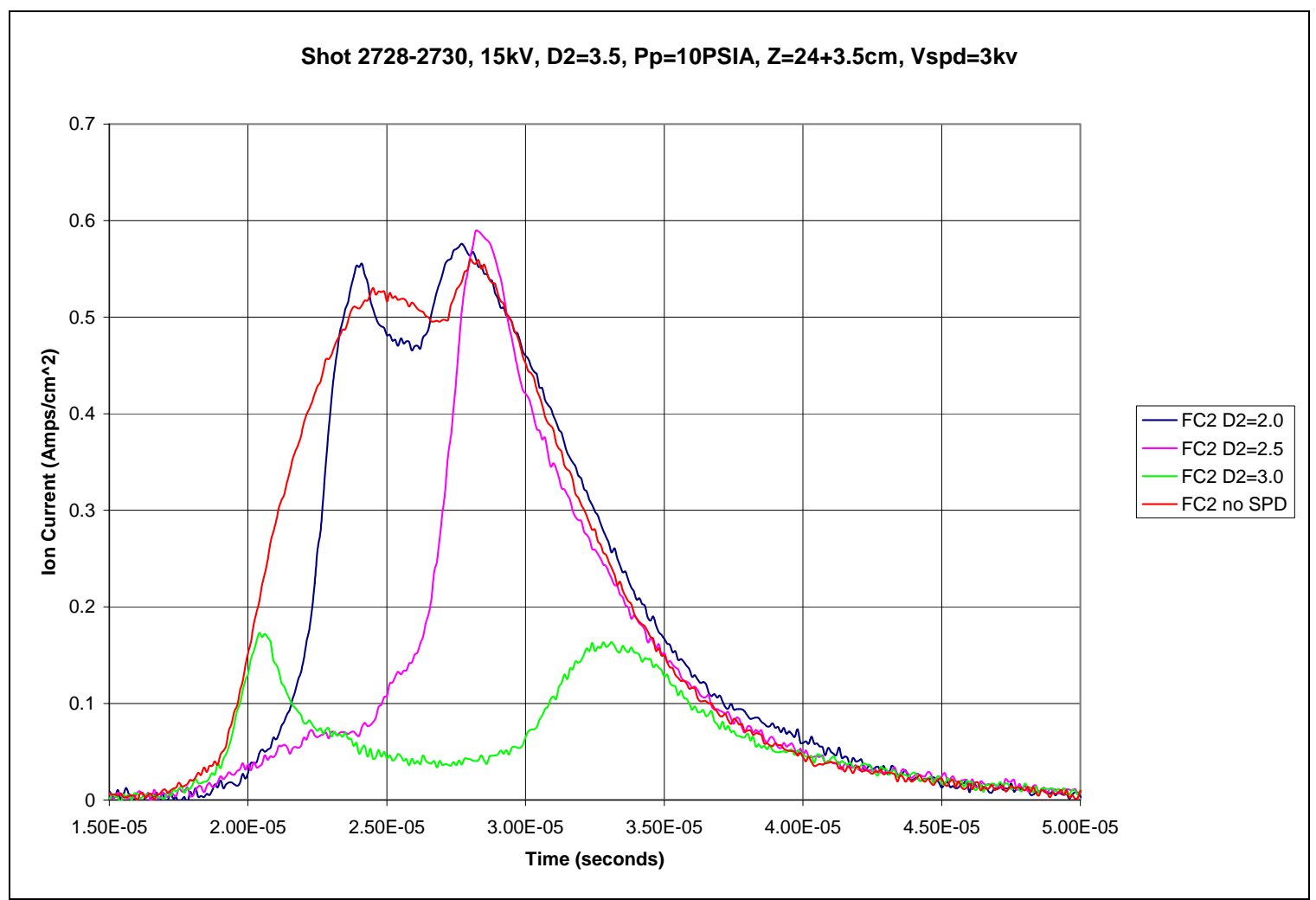

Figure 3.2.3: Additional Modulation of the Streaming Plasma 


\subsection{Performance of Phase II Source}

As mentioned previously, the new valve design improved the azimuthal uniformity of the source. Figure 3.3.1 shows an angular scan of the ion flux at a radius of 2.25" at a distance of $16 \mathrm{~cm}$ from the source using the new axial valve. The angles not shown were blocked by structure located between the faraday cup and the source. This data represents an azimuthal uniformity of around $\pm 10 \%$. This is a vast improvement over the results reported in Phase I.

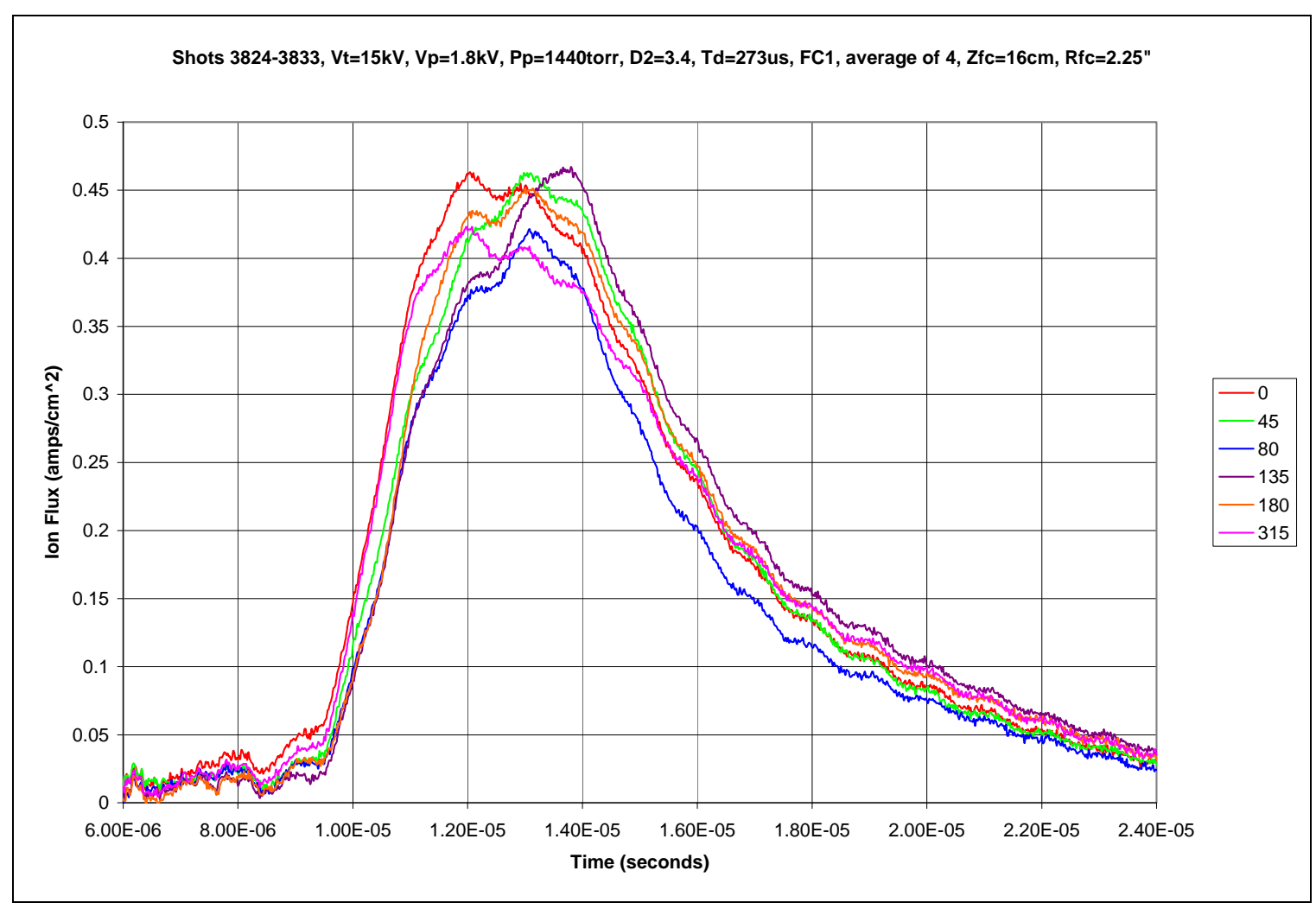

Figure 3.3.1: Azimuthal Uniformity of the Phase II Source

\subsubsection{Spectral Analysis of the Phase II Source}

An Avantes Spectrometer was rented for the purpose of analyzing the spectral lines emitted from the ion source. These data showed many interesting results.

As driving coil voltage is increased, from $11 \mathrm{kV}$ up to $15 \mathrm{kV}$, the plasma current density is not significantly higher at optimum timings, so it is reasonable to suppose that the the degree of ionization of the neutral gas in the breakdown region is approaching $100 \%$. A spectral scan at different timings shows the differences in plasma composition. 


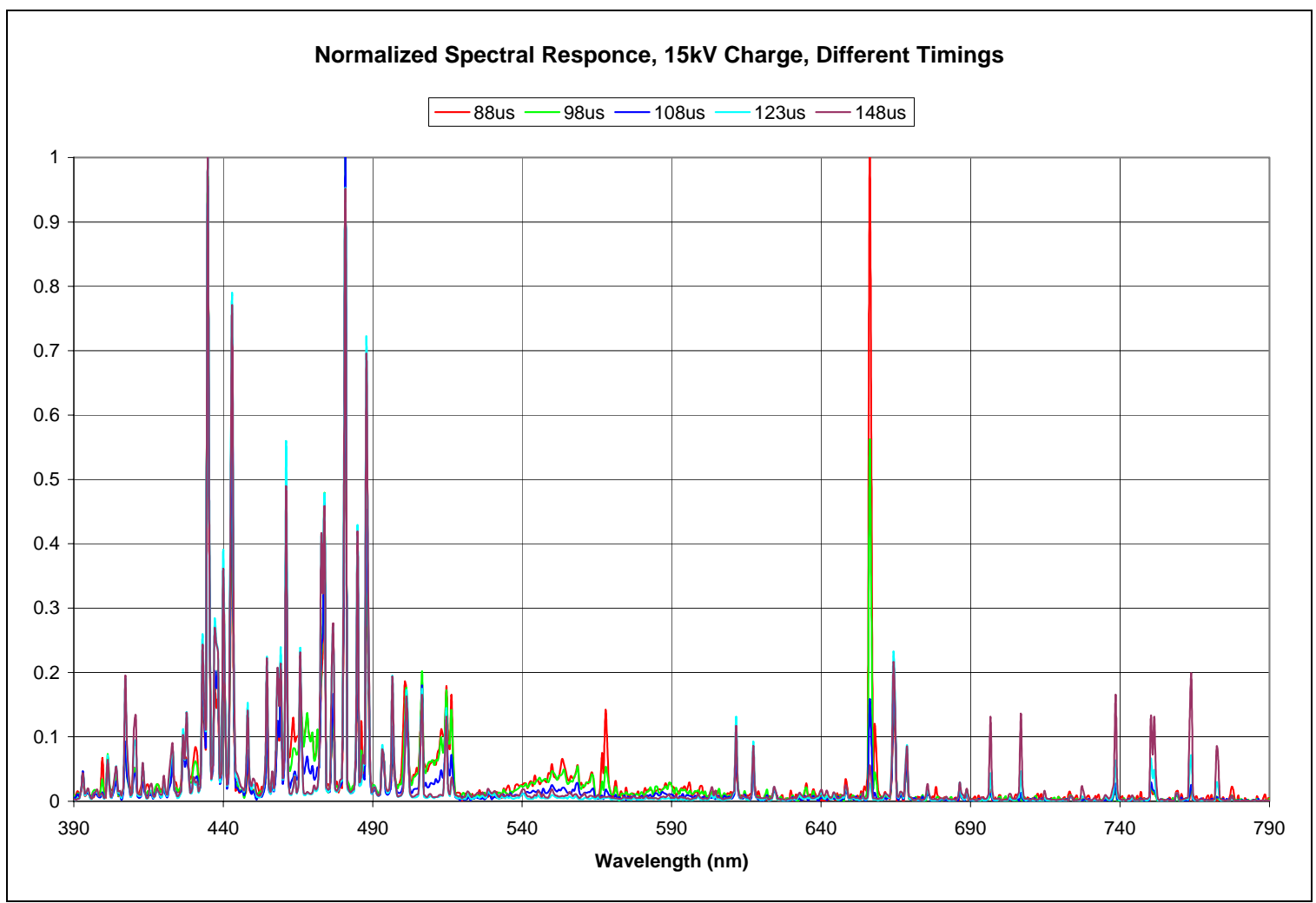

Figure 3.3.1.1: Normalized Spectrum of Plasma Source at Different Timings between Gas Injection and Driver Current

The above graph shows the normalized intensity versus wavelength for the different timings. For wavelengths between $390 \mathrm{~nm}$ and around $500 \mathrm{~nm}$, the response is entirely from $\mathrm{Ar}^{1+}$. Between $490 \mathrm{~nm}$ and $640 \mathrm{~nm}$, aside from the lines at $611.5 \mathrm{~nm}, 617.2 \mathrm{~nm}$, and 624.3nm, which are all $\mathrm{Ar}^{1+}$, the lines are from a mixture of $\mathrm{C}, \mathrm{N}$, and $\mathrm{O}$. For example, the line at $568 \mathrm{~nm}$ is from $\mathrm{N}^{1+}$. These lines are more intense normalized for the earlier timings, but are about equivalent in magnitude for all timings as can be seen the next graph. These are small as compared to a raw reading intensity of 18000 for the later timings. The more significant line is the $656.3 \mathrm{~nm}$, which is a $\mathrm{H}$ line. Again, the relative ratio shows it to be more significant for the earlier timings. At the two early timings, the raw reading was around 1400 while at the three later timings the raw reading was around 500. The $\mathrm{H}$ can originate from many sources including hydrocarbons used in plastics and oils. The change in intensity implies that $\mathrm{H}$ gas in the breakdown region is being displaced by Ar. In all cases, some of the spectral response is caused by absorption by the gas in the vacuum and the window between the vacuum and the fiber optic cable to the spectrometer. 


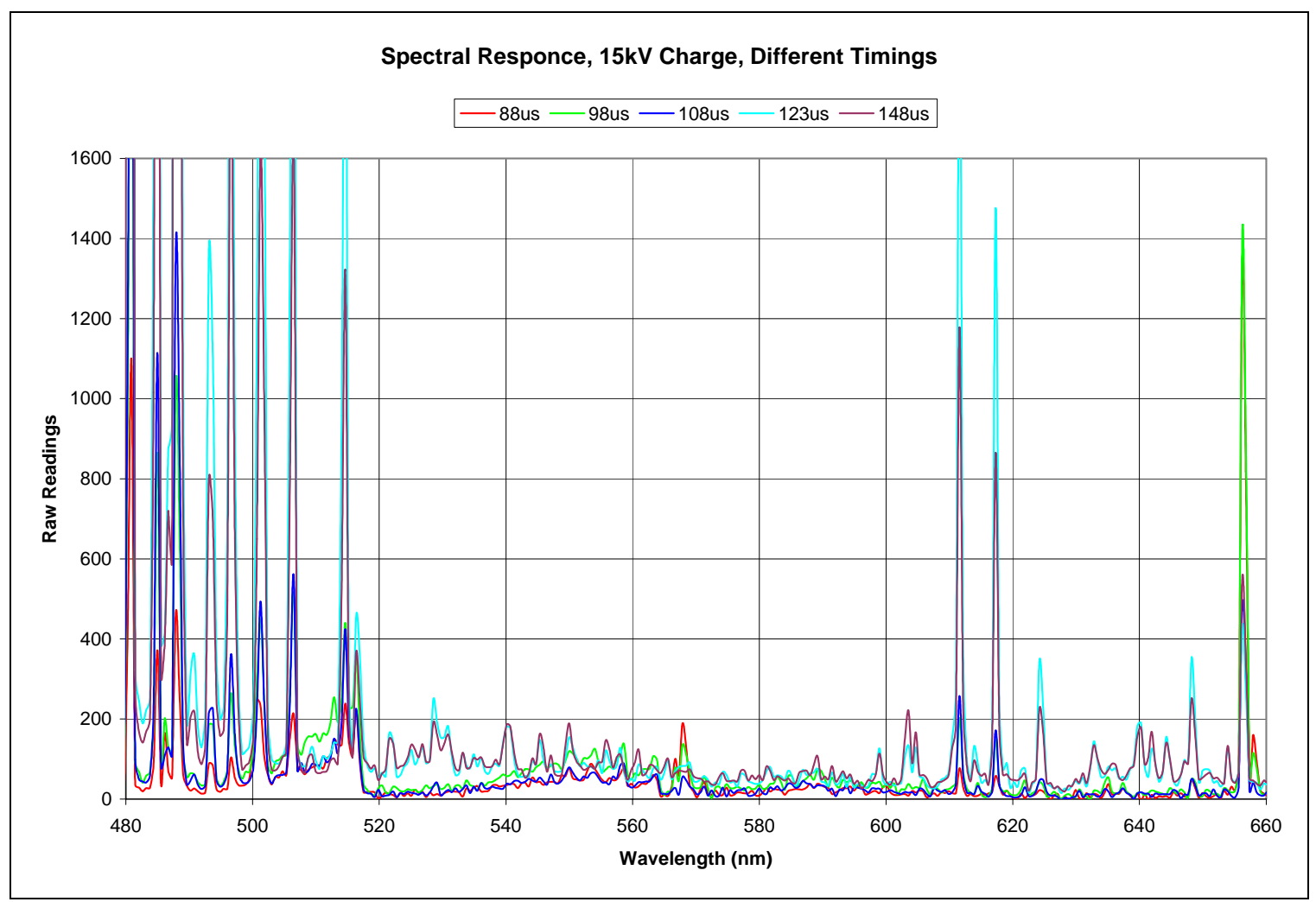

Figure 3.3.1.2: Spectrum of Plasma Source at Different Timings between Gas Injection and Driver Current Focusing on Non-Ar Lines

The later lines between $690 \mathrm{~nm}$ and $800 \mathrm{~nm}$ are mostly from neutral Ar, which has a significant number of strong lines in that range. The spectrometer did not have sufficient range to review the many $\mathrm{Ar}^{2+}$ lines in the range below 300nm. However, there are a few $\mathrm{Ar}^{2+}$ lines in the $300 \mathrm{~nm}$ to $415 \mathrm{~nm}$ range. One set of $\mathrm{Ar}^{2+}$ lines occurs at $328.5 \mathrm{~nm}$, $330.2 \mathrm{~nm}, 331.1 \mathrm{~nm}, 333.6 \mathrm{~nm}, 334.5 \mathrm{~nm}$, and $335.9 \mathrm{~nm}$. Another set of $\mathrm{Ar}^{2+}$ lines is at $348.1 \mathrm{~nm}, 350 \mathrm{~nm}$, and $350.4 \mathrm{~nm}$. Finally, $\mathrm{Ar}^{2+}$ lines appear at $379.5 \mathrm{~nm}$ and $385.8 \mathrm{~nm}$. None of the lines in this range is more than $0.25 \%$ that of the $\mathrm{Ar}^{+}$line intensities which is mostly in the level of the noise, but voids exist at $330 \mathrm{~nm}, 350 \mathrm{~nm}$, and $380 \mathrm{~nm}$, where some $\mathrm{Ar}^{2+}$ lines should be expected. Therefore, there is no strong evidence that any of these lines appear in the spectral response from the ion source, providing good evidence that no significant amount of $\mathrm{Ar}^{2+}$ is being produced by this source. 


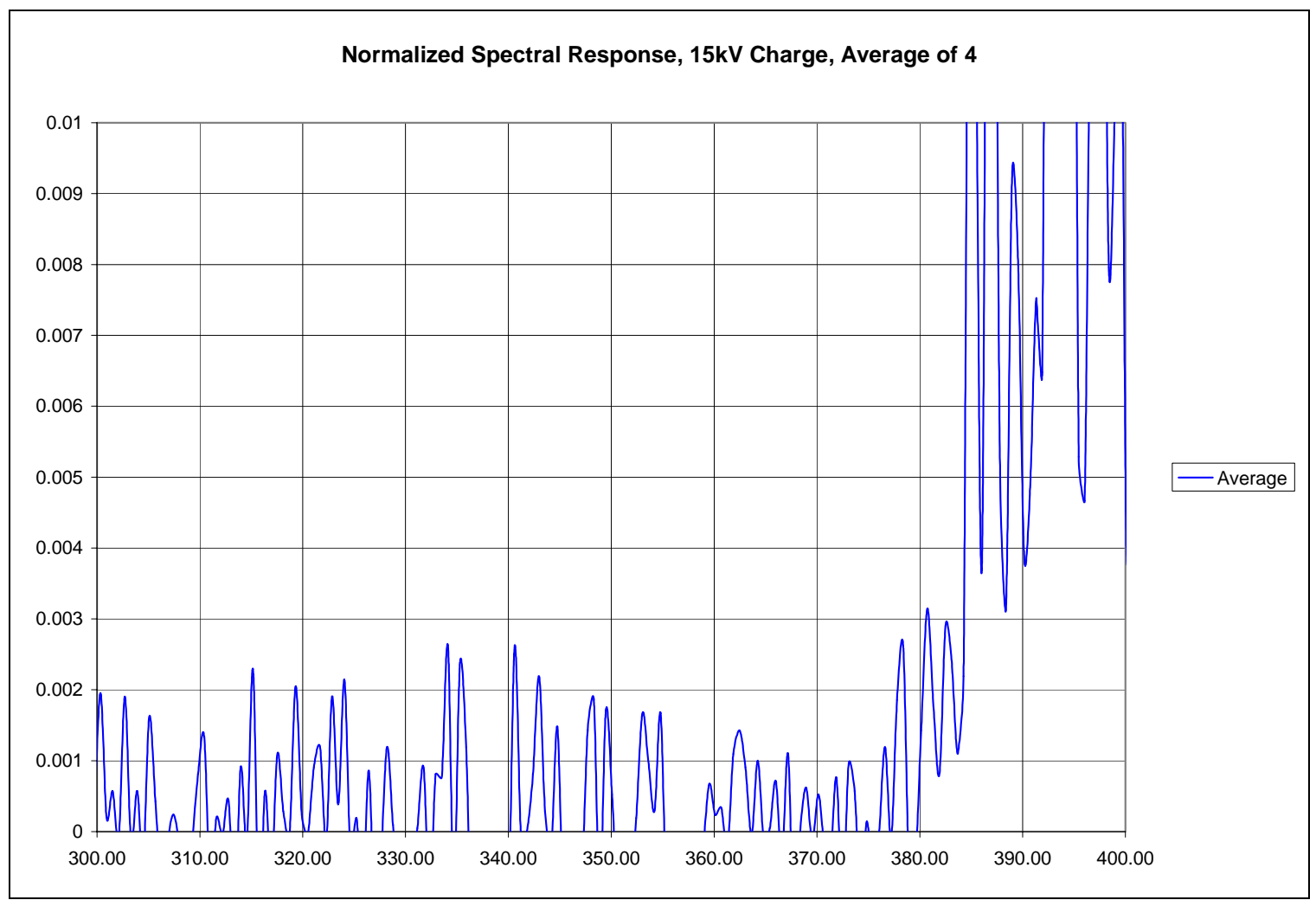

Figure 3.3.1.3: Normalized Spectrum of Plasma Source at Different Timings between Gas Injection and Driver Current for wavelengths where lines of $\mathrm{Ar}^{2+}$ would occur.

The important information discovered from this testing is the proper timing for the ion source. The critical factors, as we have described, are that at too early times there is insufficient neutral gas to break down, and at too late times there is an excessive amount of neutral gas downstream from the breakdown region that will attenuate the ion beam. The best time is one of sufficient gas in the breakdown region without too much gas downstream. At the early times, $88 \mu$ s and $98 \mu$ s, there does not seem to be enough Ar, as the $\mathrm{H}$ peak is significant both in absolute magnitude and normalized. The later times, $123 \mu \mathrm{s}$ and $148 \mu \mathrm{s}$, show larger normalized neutral Ar peaks. This would imply a large amount of neutral gas outside the breakdown region. So the best timing, as determined by this data, would be $108 \mu \mathrm{s}$. This corresponded to the best timing as determined by Faraday cup measurements of the ion beam current density $20-30 \mathrm{~cm}$ downstream.

Another correlation can be made using the relative intensity information provided by NIST at http://physics.nist.gov/cgi-bin/AtData/main asd of the $\mathrm{Ar}^{1+}$ lines to those of the normalized spectral response of the ion source. The following table correlates several of the $\mathrm{Ar}^{1+}$ lines for the $123 \mu \mathrm{s}$ timing. I have chosen only the strong lines that are sufficiently separated from other lines not to cause a resolution problem for the spectrometer. 
Figure 3.3.1.4: Table Comparing NIST Relative Intensity and Spectral Data for $\mathrm{Ar}^{1+}$

\begin{tabular}{|c|c|c|}
\hline $\mathrm{Ar}^{1+}$ Spectral Line & Relative Intensity (NIST) & Normalized Intensity (Data) \\
\hline $401.4 \mathrm{~nm}$ & 50 & 0.07 \\
\hline $407.2 \mathrm{~nm}$ & 200 & 0.19 \\
\hline $410.4 \mathrm{~nm}$ & 150 & 0.09 \\
\hline $413.2 \mathrm{~nm}$ & 300 & 0.06 \\
\hline $434.8 \mathrm{~nm}$ & 800 & 1.00 \\
\hline $448 \mathrm{~nm}$ & 200 & 0.15 \\
\hline $454.5 \mathrm{~nm}$ & 400 & 0.22 \\
\hline $461 \mathrm{~nm}$ & 550 & 0.56 \\
\hline $476.5 \mathrm{~nm}$ & 800 & 0.26 \\
\hline $480.6 \mathrm{~nm}$ & 550 & 0.94 \\
\hline $484.8 \mathrm{~nm}$ & 150 & 0.43 \\
\hline $488 \mathrm{~nm}$ & 800 & 0.72 \\
\hline $493.3 \mathrm{~nm}$ & 35 & 0.09 \\
\hline $496.5 \mathrm{~nm}$ & 200 & 0.19 \\
\hline $506.2 \mathrm{~nm}$ & 70 & 0.17 \\
\hline $611.5 \mathrm{~nm}$ & 100 & 0.13 \\
\hline $617.2 \mathrm{~nm}$ & 150 & 0.09 \\
\hline $664.4 \mathrm{~nm}$ & 50 & 0.23 \\
\hline $668.4 \mathrm{~nm}$ & 35 & 0.09 \\
\hline
\end{tabular}

While the correlation between the relative intensity from NIST and the normalized intensity from the data is not exact, they do correspond. All strong peaks listed in the NIST data appear in the spectral results from the ion source. In general, the stronger lines from the NIST data do appear as stronger lines in the data from the ion source.

The spectrometer was also used to measure the spectrum from the ion source when other voltages were used. Following is a graph showing the normalized values from three different charges, $11 \mathrm{kV}, 13 \mathrm{kV}$, and $15 \mathrm{kV}$ at a timing of $223 \mu \mathrm{s}$. This timing is late enough that the plasma chamber is filled with Ar. As can be seen, the results show nearly the same spectrum from the source for all three voltages. The apparent shift in the entire spectrum is an instrumental artifact associated with the spectrometer. 


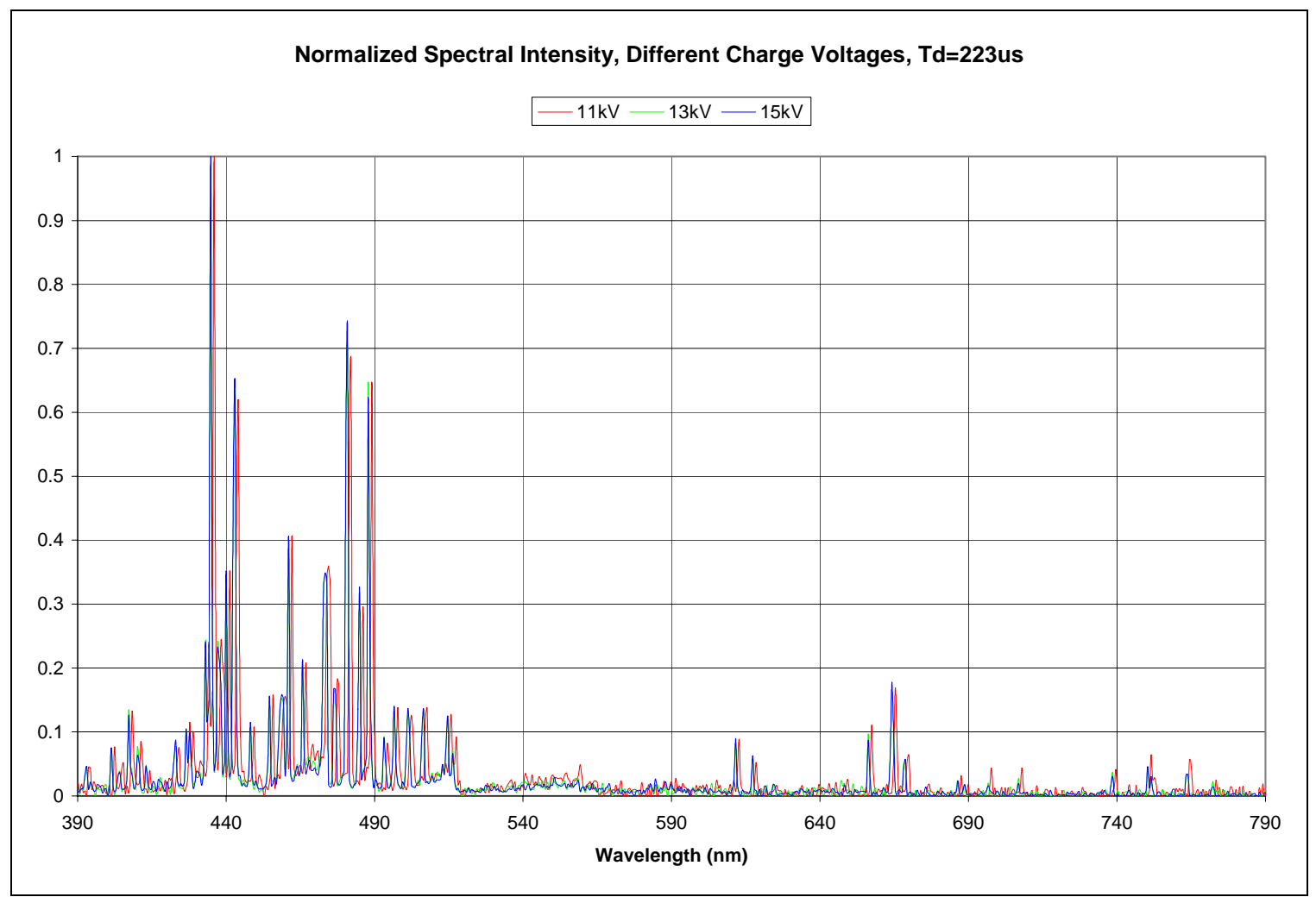

Figure 3.3.1.5: Normalized Spectrum of Plasma Source at Different Source Driver Charge Voltages

The differences in the magnitudes between the 3 voltages can be seen in the following graph. Using the largest peak, that at the $434.8 \mathrm{~nm}$ line, the magnitude at $15 \mathrm{kV}$ was about 2500 , at $13 \mathrm{kV}$ it was about 1700 , and at $11 \mathrm{kV}$ it was about $930.11 \mathrm{kV}$ to $13 \mathrm{kV}$ is approximately a $40 \%$ increase in energy, while the intensity difference is about $83 \%$. From $13 \mathrm{kV}$ to $15 \mathrm{kV}$ represents approximately a $33 \%$ increase in energy and had a $47 \%$ increase in intensity. This shows that the increase in intensity at the higher voltages is becoming closer in proportion to the change in energy, implying that the gas in the breakdown region is approaching 100\% ionized at the higher voltage. 


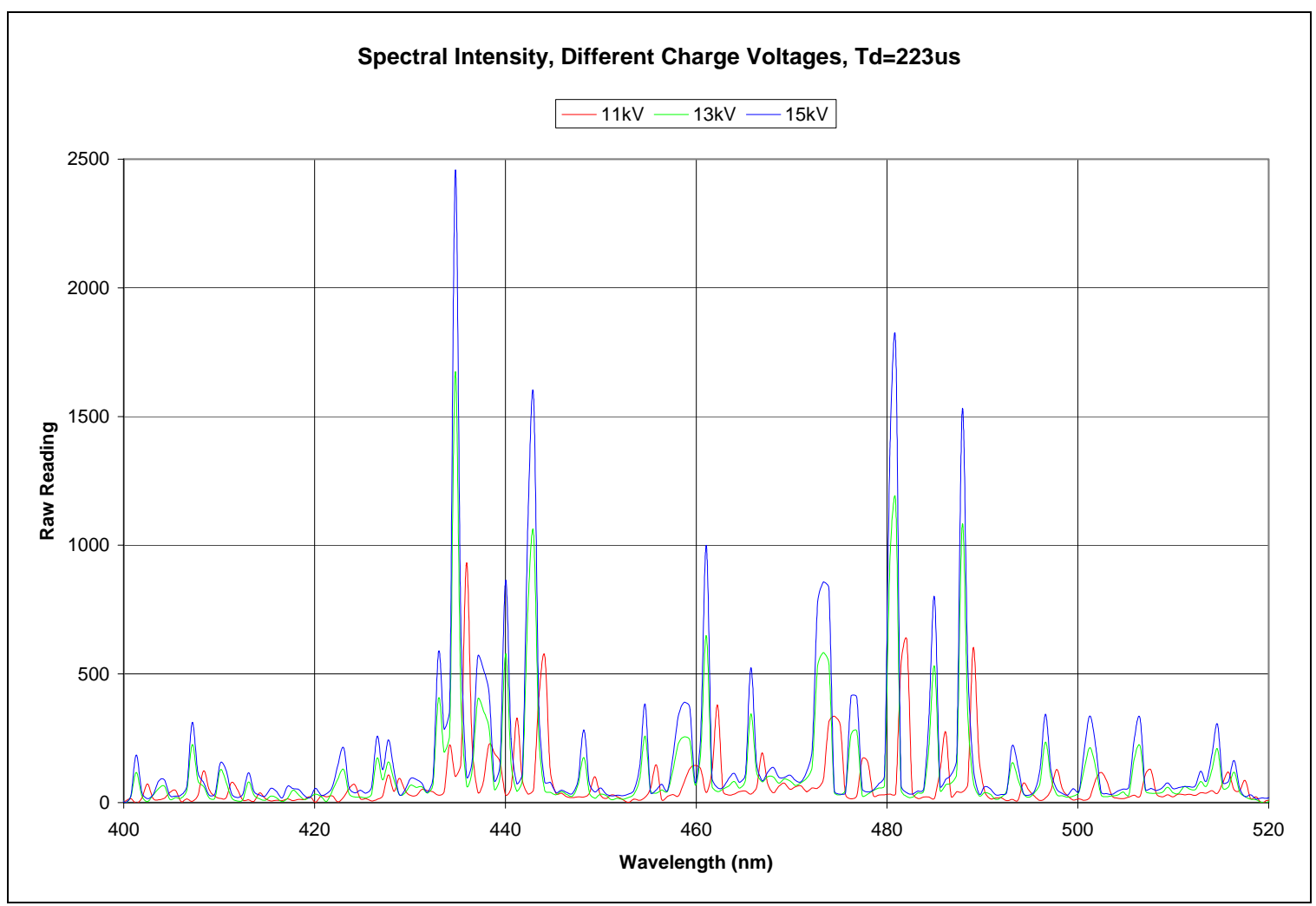

Figure 3.3.1.6: Spectrum of Plasma Source at Different Source Driver Charge Voltages

Another measurement was done looking at the plasma from the side versus the front to make sure that there were no differences in plasma structure affecting the results. The next two graphs show that there was no noticeable difference in the spectrum from the two directions, although the side view showed lower normalized magnitudes in many of the $\mathrm{Ar}^{1+}$ lines while demonstrating a small increase in magnitude in the neutral Ar lines. That difference is probably associated with the design of the nozzle used to move gas from the valve to the breakdown region. The gas is directed radially outward, thus resulting in more neutral gas at the outer edge of the plasma than in front of the plasma. The small effects of attenuation of the light through the neutral gas can account for the differences seen in the magnitudes of the different lines. 
Applied Pulsed Power, Inc.

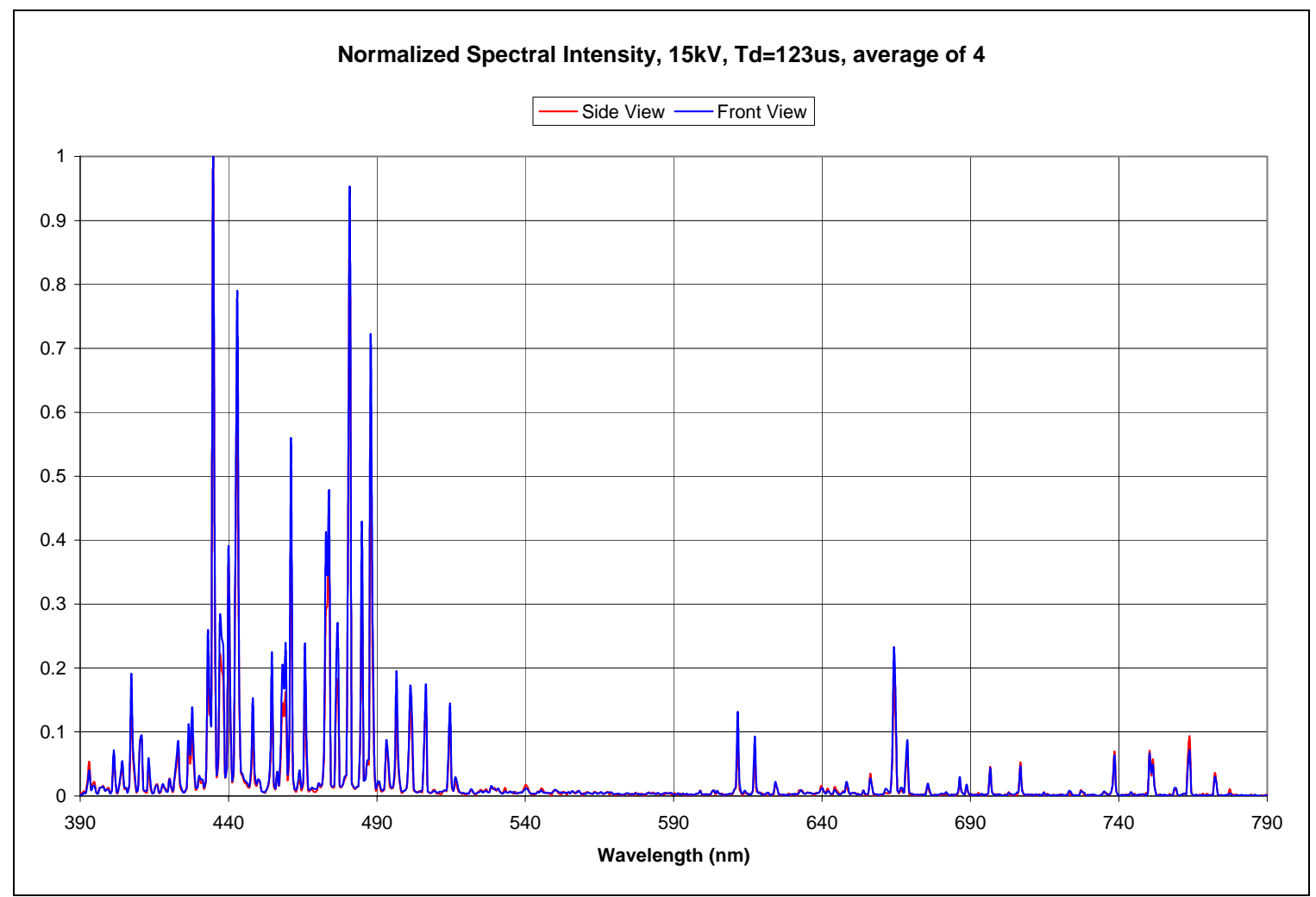

Figure 3.3.1.7: Averaged and Normalized Spectrum of Plasma Source from Side and Forward Views 


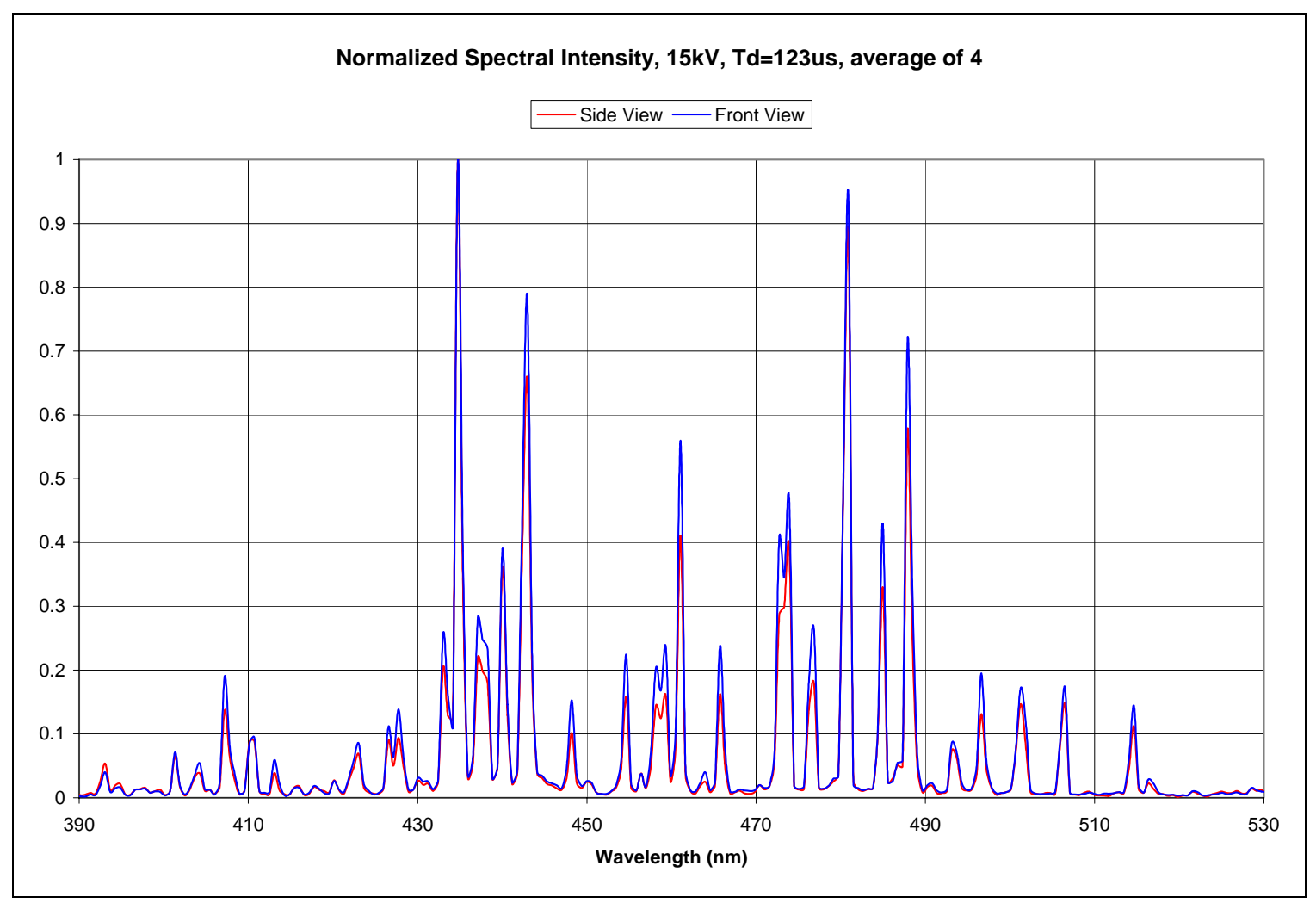

Figure 3.3.1.8: Averaged and Normalized Spectrum of Plasma Source from Side and Forward Views Focusing on $\mathrm{Ar}^{1+}$ lines

\subsubsection{Divergence Measurements of the Phase II Source}

The following Figures, 3.3.2.1 through 3.3.2.10 summarize data on the angular divergence of the ion current density in the streaming plasma. 
Applied Pulsed Power, Inc.

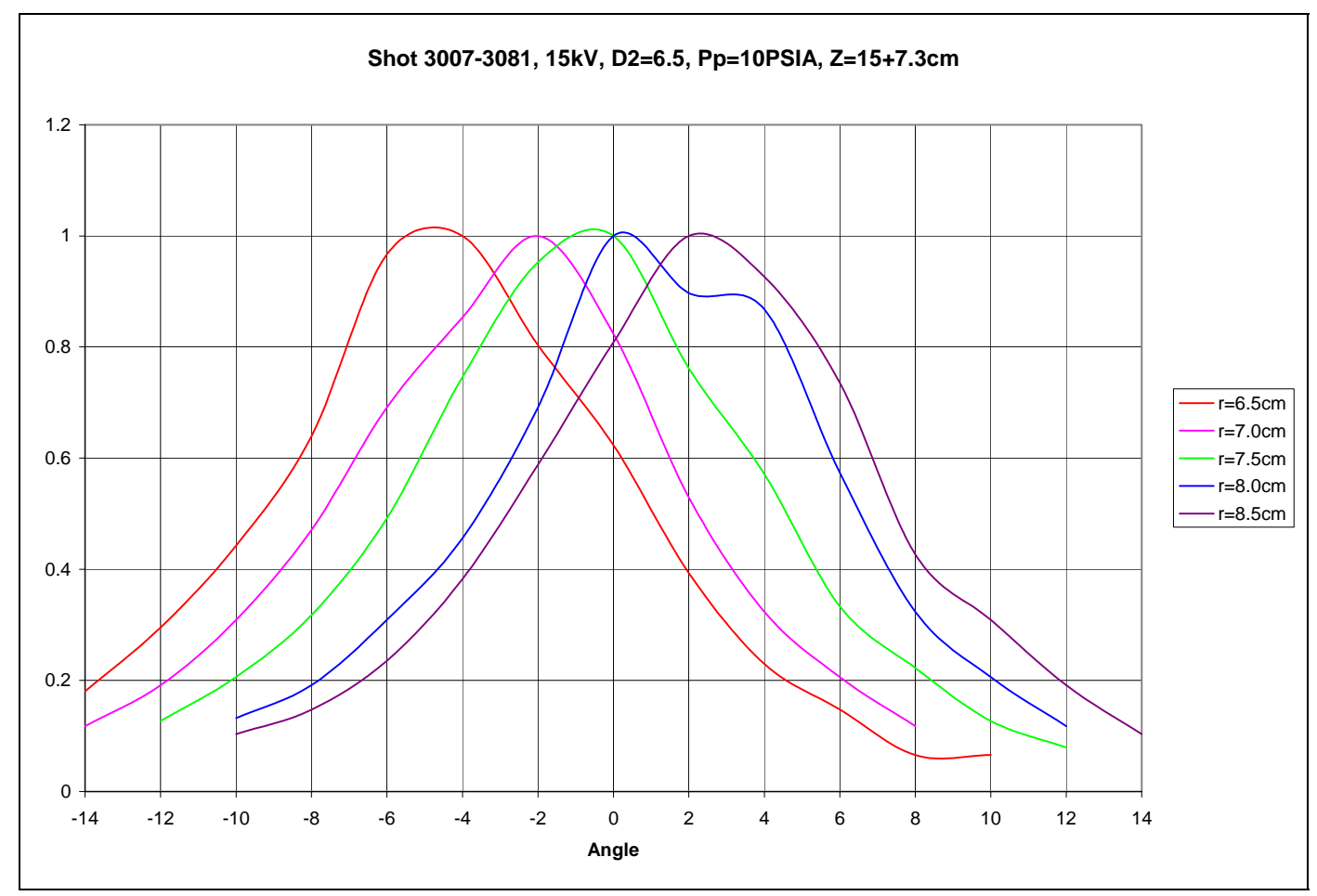

Figure 3.3.2.1: Normalized Current versus Faraday Cup Angle for Selected Radii at $22.3 \mathrm{~cm}$ from the Source

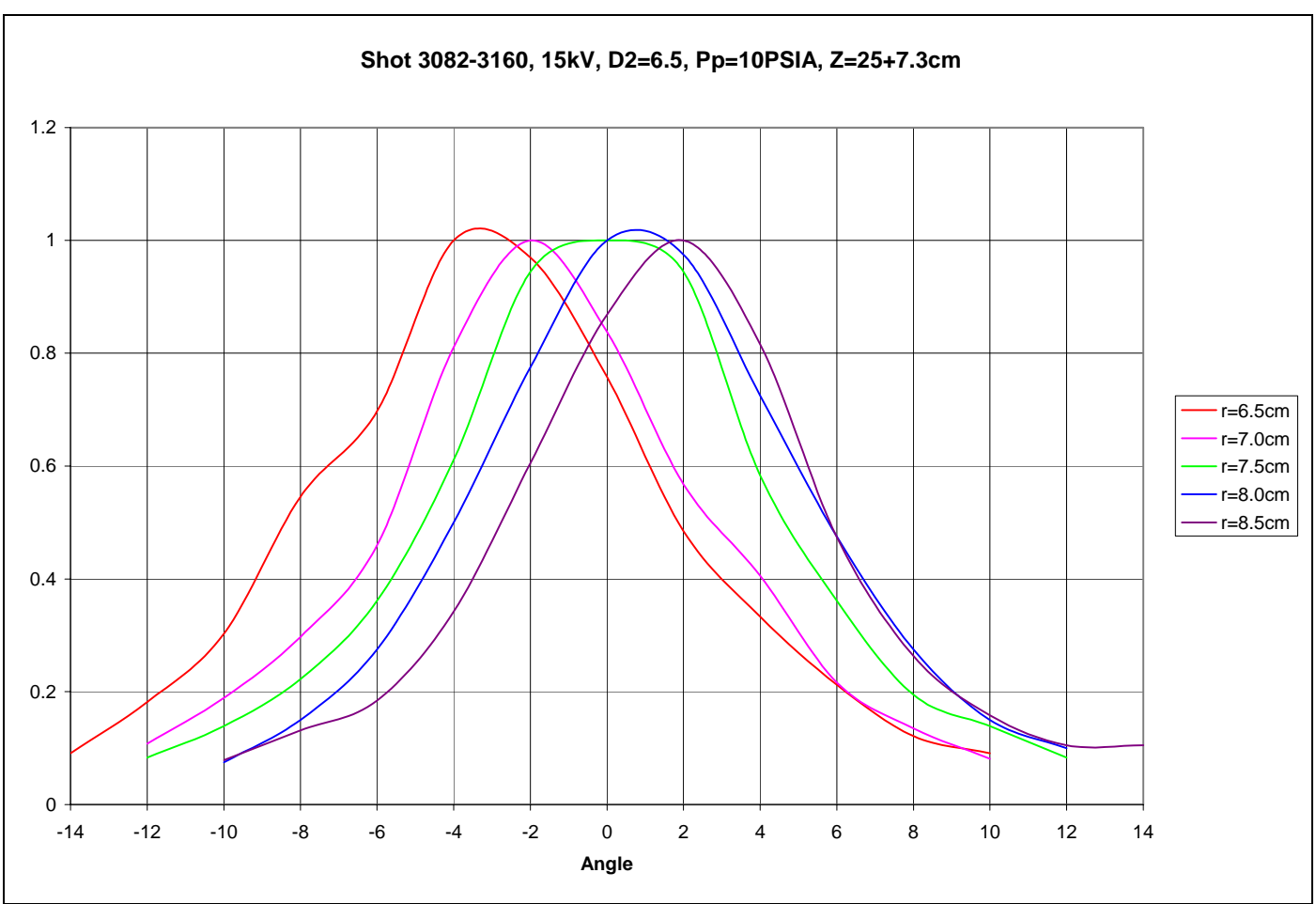

Figure 3.3.2.2: Normalized Current versus Faraday Cup Angle for Selected Radii at $32.3 \mathrm{~cm}$ from the Source 
Applied Pulsed Power, Inc.

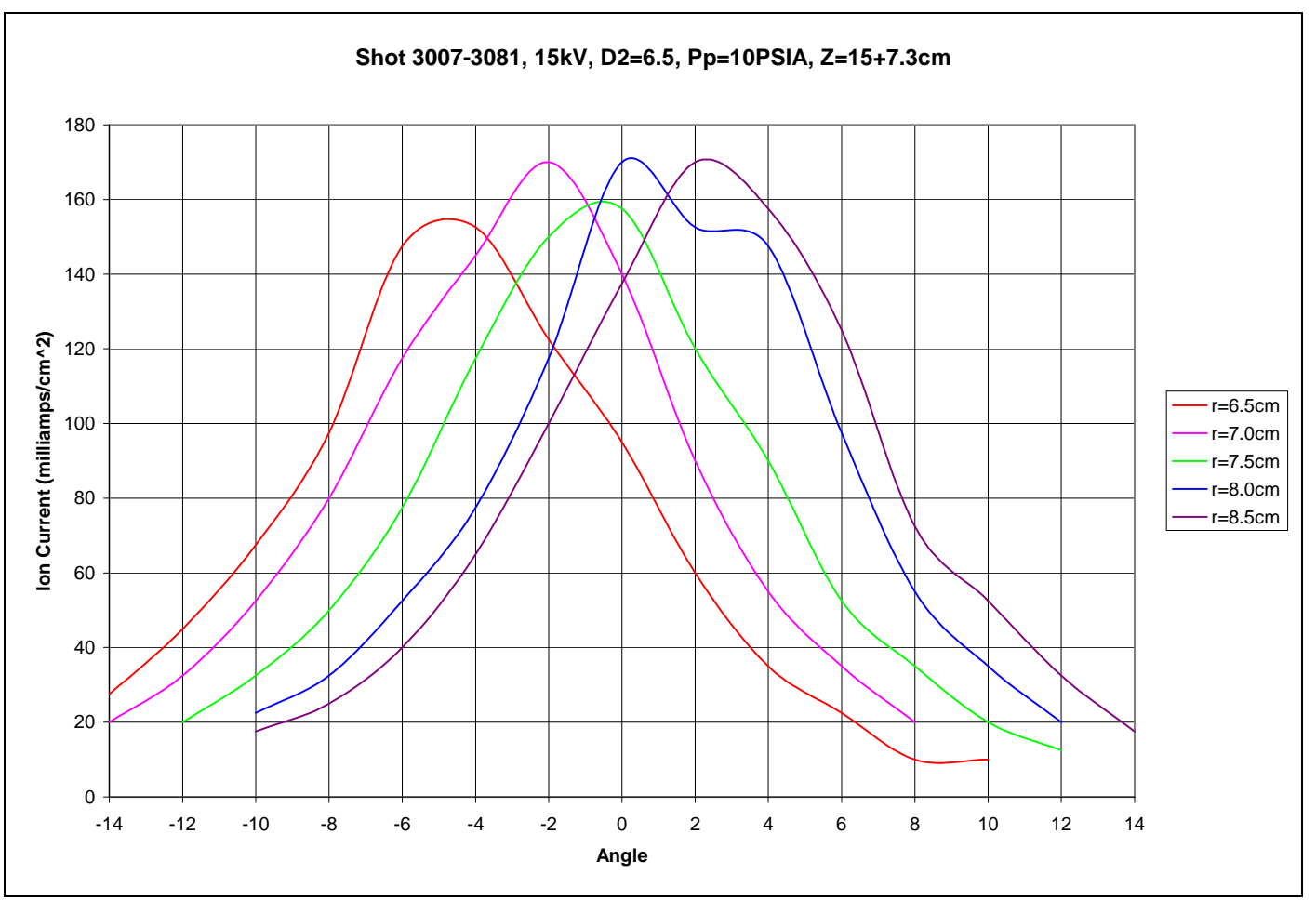

Figure 3.3.2.3: Absolute Current Density versus Faraday Cup Angle for Selected Radii at $22.3 \mathrm{~cm}$ from Source

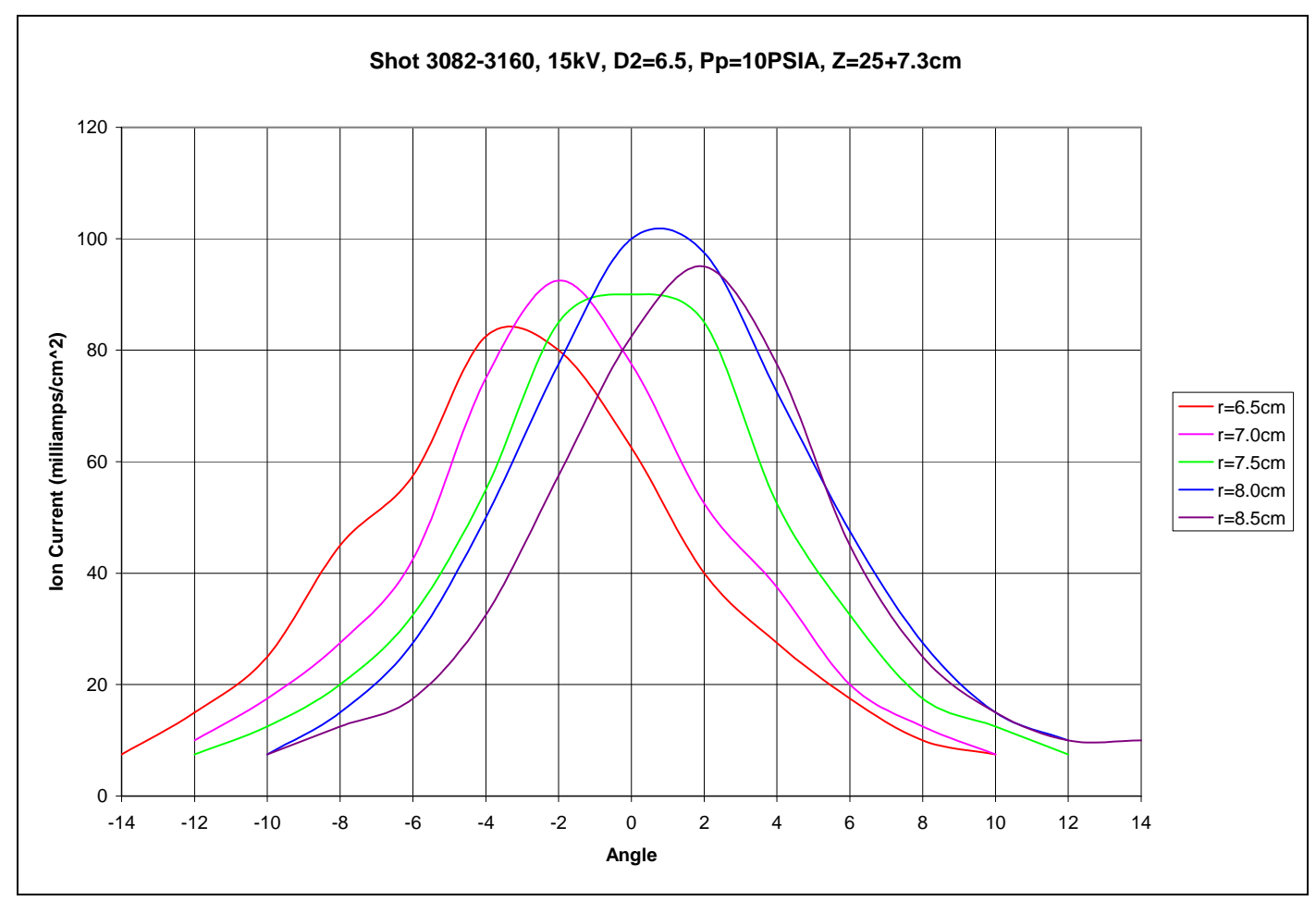

Figure 3.3.2.4: Absolute Current Density versus Faraday Cup Angle for Selected Radii at $32.3 \mathrm{~cm}$ from Source 
Applied Pulsed Power, Inc.

Figure 3.3.2.5: Single Standard Deviation Divergence Calculated Using Specified Gaussian Width

\begin{tabular}{|c|c|c|}
\hline Width & $\mathrm{Z}=22.3 \mathrm{~cm}$ & $\mathrm{Z}=33.3 \mathrm{~cm}$ \\
\hline$+/-2^{\mathrm{o}}$ & $2.8^{\mathrm{o}}$ & $-\mathrm{NA}-$ \\
\hline$+/-4^{\mathrm{o}}$ & $3.3^{\mathrm{o}}$ & $2.2^{\mathrm{o}}$ \\
\hline$+/-6^{\mathrm{o}}$ & $3.7^{\mathrm{o}}$ & $3.1^{\mathrm{o}}$ \\
\hline$+/-8^{\mathrm{o}}$ & $4.1^{\mathrm{o}}$ & $3.7^{\mathrm{o}}$ \\
\hline$+/-10^{\mathrm{o}}$ & $4.6^{\mathrm{o}}$ & $4.3^{\mathrm{o}}$ \\
\hline
\end{tabular}

Figure 3.3.2.6: Observed Angle of Peak Ion Current versus Radius

\begin{tabular}{|c|c|c|}
\hline Radius & $\mathrm{Z}=22.3 \mathrm{~cm}$ & $\mathrm{Z}=33.3 \mathrm{~cm}$ \\
\hline $6.0 \mathrm{~cm}$ & $-\mathrm{NA}-$ & $-4.0^{\circ}$ \\
\hline $6.5 \mathrm{~cm}$ & $-4.8^{\circ}$ & $-3.5^{\circ}$ \\
\hline $7.0 \mathrm{~cm}$ & $-2.0^{\circ}$ & $-2.0^{\circ}$ \\
\hline $7.5 \mathrm{~cm}$ & $-0.6^{\circ}$ & $0.0^{\circ}$ \\
\hline $8.0 \mathrm{~cm}$ & $0.2^{\circ}$ & $0.8^{\circ}$ \\
\hline $8.5 \mathrm{~cm}$ & $2.2^{\circ}$ & $1.9^{\circ}$ \\
\hline $9.0 \mathrm{~cm}$ & $5.0^{\circ}$ & $-\mathrm{NA}-$ \\
\hline $9.5 \mathrm{~cm}$ & $-\mathrm{NA}-$ & $2.2^{\circ}$ \\
\hline
\end{tabular}

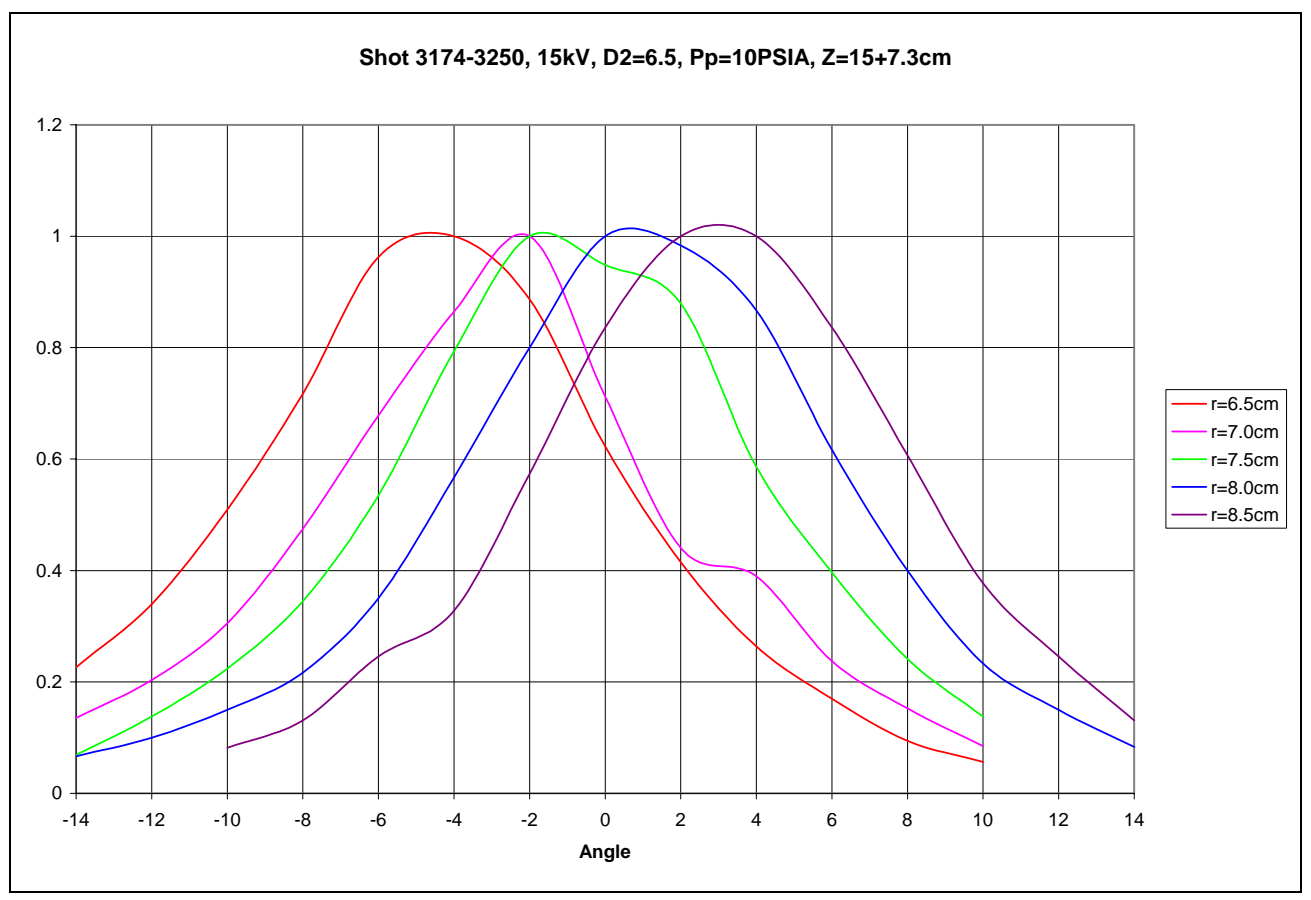

Figure 3.3.2.7: Normalized Peak Ion Current versus Faraday Cup Angle for Selected Radii at $22.3 \mathrm{~cm}$ from Modulated Source 


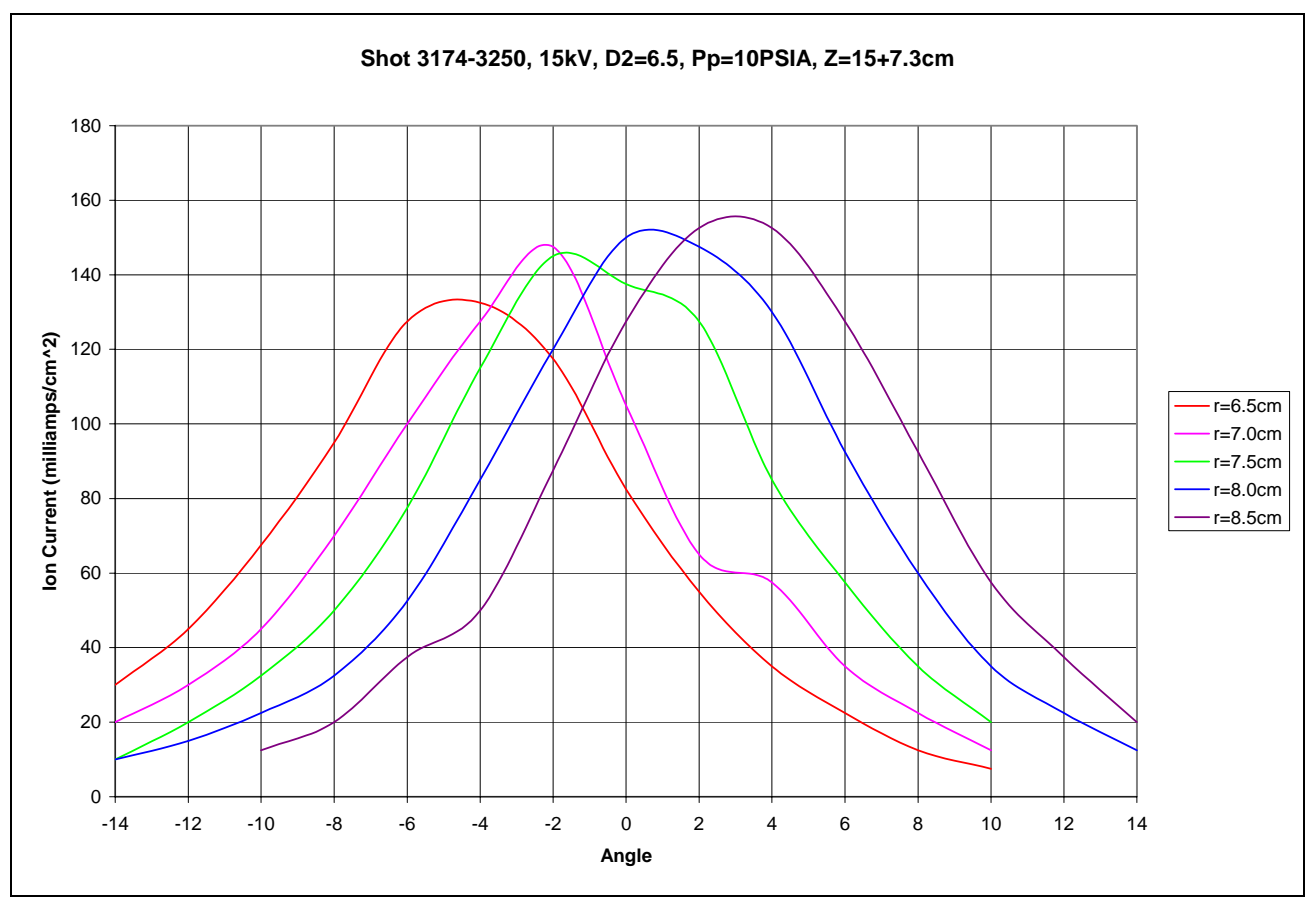

Figure 3.3.2.8: Peak Ion Current versus Faraday Cup Angle for Selected Radii at $22.3 \mathrm{~cm}$ from Modulated Source

Figure 3.3.2.9: Single Standard Deviation Divergence Calculated Using Specified Gaussian Width (modulated versus un-modulated)

\begin{tabular}{|c|c|c|}
\hline Width & $Z=22.3 \mathrm{~cm}$ & $\begin{array}{c}Z=22.3 \mathrm{~cm} \\
\text { (modulated) }\end{array}$ \\
\hline$+/-2^{\mathrm{o}}$ & $2.8^{\mathrm{o}}$ & $4.6^{\mathrm{o}}$ \\
\hline$+/-4^{\mathrm{o}}$ & $3.3^{\mathrm{o}}$ & $3.9^{\mathrm{o}}$ \\
\hline$+/-6^{\mathrm{o}}$ & $3.7^{\mathrm{o}}$ & $4.2^{\mathrm{o}}$ \\
\hline$+/-8^{\mathrm{o}}$ & $4.1^{\mathrm{o}}$ & $4.3^{\mathrm{o}}$ \\
\hline$+/-10^{\mathrm{o}}$ & $4.6^{\mathrm{o}}$ & $4.85^{\mathrm{o}}$ \\
\hline
\end{tabular}

Figure 3.3.2.10: Observed Angle of Peak Ion Current versus Radius (modulated versus un-modulated)

\begin{tabular}{|c|c|c|}
\hline Radius & $\mathrm{Z}=22.3 \mathrm{~cm}$ & $\begin{array}{c}\mathrm{Z}=22.3 \mathrm{~cm} \\
\text { (modulated) }\end{array}$ \\
\hline $6.0 \mathrm{~cm}$ & $-\mathrm{NA}-$ & $-6.1^{\mathrm{o}}$ \\
\hline $6.5 \mathrm{~cm}$ & $-4.8^{\mathrm{o}}$ & $-4.5^{\mathrm{o}}$ \\
\hline $7.0 \mathrm{~cm}$ & $-2.0^{\mathrm{o}}$ & $-2.2^{\mathrm{o}}$ \\
\hline $7.5 \mathrm{~cm}$ & $-0.6^{\mathrm{o}}$ & $-1.6^{\mathbf{0}}$ \\
\hline $8.0 \mathrm{~cm}$ & $0.2^{\mathrm{o}}$ & $0.6^{\mathrm{o}}$ \\
\hline $8.5 \mathrm{~cm}$ & $2.2^{\mathrm{o}}$ & $3.0^{\mathrm{o}}$ \\
\hline $9.0 \mathrm{~cm}$ & $5.0^{\mathrm{o}}$ & $-\mathrm{NA}-$ \\
\hline
\end{tabular}


It is seen that the angular divergence of the ion beam is increased somewhat by passing through the modulator, but mainly the angular distribution is made more nearly Gaussian (the calculated divergence is more independent of the chosen angle) by the modulator. Thus the penalty in beam divergence caused by use of the modulator is very slight.

\subsubsection{Time of Flight Analysis of the Phase II Source}

An axially and azimuthally adjustable faraday cup was used for time of flight analysis on the ion beam. The single faraday cup was employed to assure greater accuracy in the analysis. The azimuthal variation with the Phase II source was small, so a single angle was chosen. Three different axial positions were chosen, $13.3 \mathrm{~cm}, 23.5 \mathrm{~cm}$, and $33.6 \mathrm{~cm}$.

Figure 3.3.3.1 shows the faraday cup current measured at the three different axial locations. As indicated, there is significant temporal broadening of the Gaussian curve at the further axial locations. This indicates a distribution in ion velocity. As seen in figure 3.3 .3 .3 , the velocity of the wavefront is about $4.1 \mathrm{~cm} / \mu \mathrm{s}$ while the velocity of the peak is around $1.5 \mathrm{~cm} / \mu \mathrm{s}$. These velocities correlate to kinetic energies of $348 \mathrm{eV}$ and $46.6 \mathrm{eV}$ respectively. This is the energy imparted by the jxB force of the source driver on the plasma.

It can be understood from the comparative data that the ions seem to be slowing down, as the velocities between the further two positions are slower than for the shorter two positions. As speculated previously, this slowing is the result of the interactions of the plasma with neutral gas located downstream of the source.

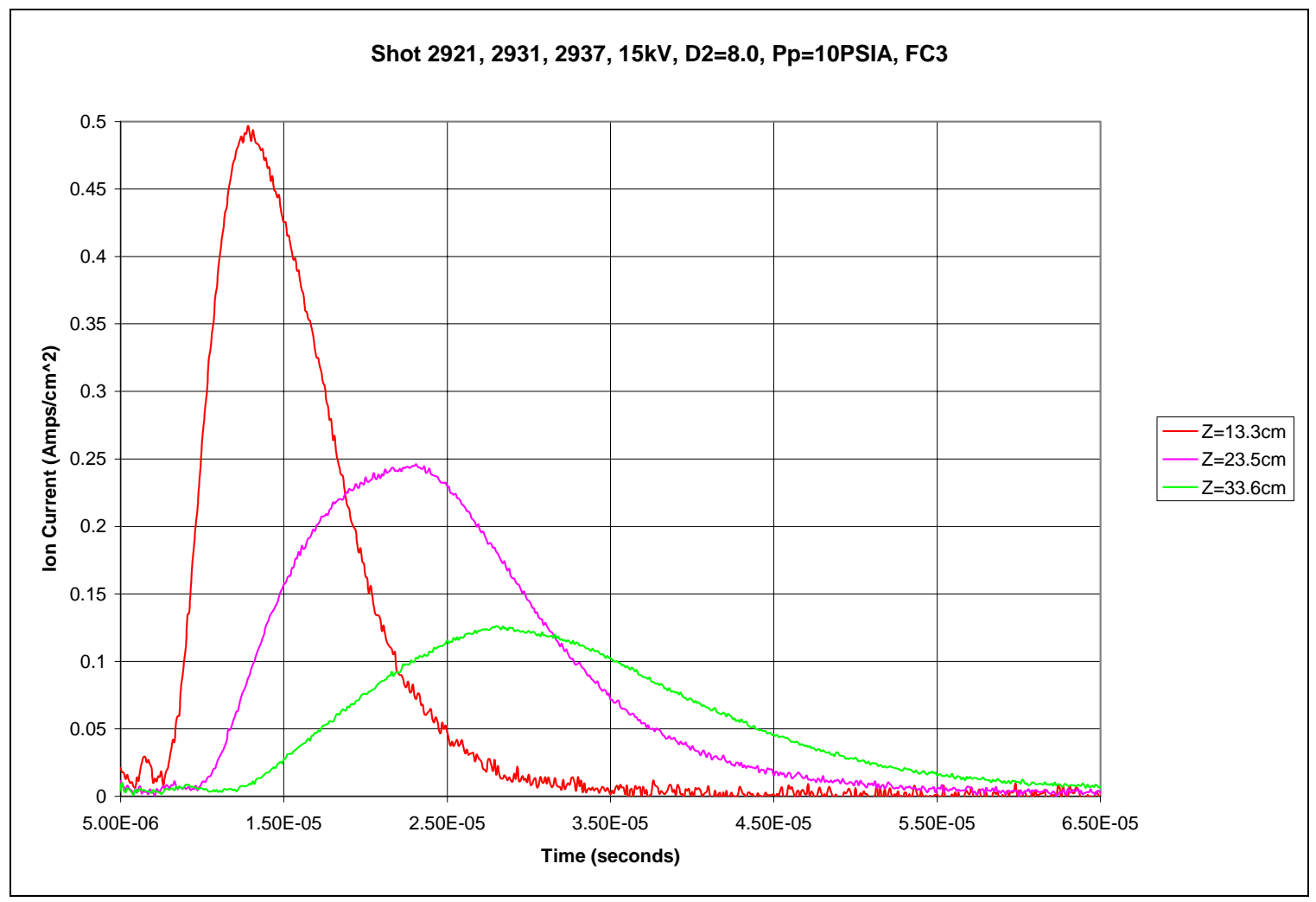


Figure 3.3.3.1: Ion Current versus Time at Different Distances from Source

Figure 3.3.3.2: Measured Time to Specified Ion Current Level

\begin{tabular}{|c|c|c|c|}
\hline $\begin{array}{c}\text { Percent on } \\
\text { Rise }\end{array}$ & $Z=13.3 \mathrm{~cm}$ & $Z=23.5 \mathrm{~cm}$ & $Z=33.6 \mathrm{~cm}$ \\
\hline $10 \%$ & $8.4 \mu \mathrm{s}$ & $10.9 \mu \mathrm{s}$ & $13.3 \mu \mathrm{s}$ \\
\hline $50 \%$ & $9.9 \mu \mathrm{s}$ & $13.8 \mu \mathrm{s}$ & $18.5 \mu \mathrm{s}$ \\
\hline $90 \%$ & $11.6 \mu \mathrm{s}$ & $18.7 \mu \mathrm{s}$ & $24.9 \mu \mathrm{s}$ \\
\hline
\end{tabular}

Figure 3.3.3.3: Calculated Velocity for Specified Ion Current Level

\begin{tabular}{|c|c|c|c|}
\hline $\begin{array}{c}\text { Percent on } \\
\text { Rise }\end{array}$ & $\begin{array}{c}Z=13.3 \mathrm{~cm} \\
\text { to } 23.5 \mathrm{~cm}\end{array}$ & $\begin{array}{c}Z=23.5 \mathrm{~cm} \\
\text { to } 33.6 \mathrm{~cm}\end{array}$ & $\begin{array}{c}Z=13.3 \mathrm{~cm} \\
\text { to } 33.6 \mathrm{~cm}\end{array}$ \\
\hline $10 \%$ & $4.08 \mathrm{~cm} / \mu \mathrm{s}$ & $4.21 \mathrm{~cm} / \mu \mathrm{s}$ & $4.14 \mathrm{~cm} / \mu \mathrm{s}$ \\
\hline $50 \%$ & $2.61 \mathrm{~cm} / \mu \mathrm{s}$ & $2.15 \mathrm{~cm} / \mu \mathrm{s}$ & $2.36 \mathrm{~cm} / \mu \mathrm{s}$ \\
\hline $90 \%$ & $1.43 \mathrm{~cm} / \mu \mathrm{s}$ & $1.63 \mathrm{~cm} / \mu \mathrm{s}$ & $1.53 \mathrm{~cm} / \mu \mathrm{s}$ \\
\hline
\end{tabular}

These time of flight measurements are also able to determine the temporal origin of the ion current from the source. Using the data points and the calculated velocities, the temporal origin of the ion current at the source is $4.6 \mu \mathrm{s}$. Examining the current in the source driver, see figure 3.1.5, and the pictures of the plasma, see figures 3.1.7 to 3.1.13 this time can be correlated to events occurring in the plasma.

Breakdown of neutral gas occurs as the current in the driver reaches high peak di/dt. The initial rise in current creates the seed electrons in the gas that allows later breakdown. The first falling current slope, starting at around $2.9 \mu \mathrm{s}$, results in significant plasma creation, as seen in the pictures. The $\mathrm{B}^{\circ}$ data shown in figure 3.1.22 also show the magnetic insulation caused by the plasma at that time, causing reduction of the magnetic field at more downstream axial positions. The plasma creation reaches the peak as the plasma source driver current approaches the first zero crossing. However, this is not the temporal source of the ion current. The peak acceleration of this newly formed plasma would occur as the plasma source driver current reaches the first negative peak at about $4 \mu$ s. The next rising current slope starting at around $4.2 \mu$ s creates additional plasma as seen in the pictures. These pictures also show that the plasma has significant expansion away from the source starting from around this time. Since the direction of acceleration reverses as the current in the plasma source driver reverses, the ions would reach peak velocity at the second zero crossing at about $4.4 \mu \mathrm{s}$. This time correlates with the calculated temporal origin of the ion current.

Figure 3.3.3.4: Calculated Time of Origin of Ion Current Using Time of Flight Data

\begin{tabular}{|c|c|c|c|}
\hline & $Z=13.3 \mathrm{~cm}$ & $Z=23.5 \mathrm{~cm}$ & $Z=33.6 \mathrm{~cm}$ \\
\hline Origin Time & $4.57 \mu \mathrm{s}$ & $4.61 \mu \mathrm{s}$ & $4.57 \mu \mathrm{s}$ \\
\hline
\end{tabular}


The calculation of the temporal source of the ion current from the time of flight data therefore correlates with the observed axial expansion of the plasma from the pictures of the plasma and also with the effects the plasma source driver current would have on the ions in the plasma. 


\subsection{Accelerated Beam Experiments}

\subsection{Description of Ion Beam Acceleration System}

To further test the performance of the streaming plasma neutralized ion beam source we have developed, a $100 \mathrm{kV}$ acceleration system was added to extract an ion beam from the source. In the Phase II proposal we had envisioned performing the initial experiments using a DC source, but the $100 \mathrm{kV}$ pulsed driver was available at the time experiments began, so it was employed for all acceleration experiments. By varying the timing of the acceleration pulse, the accelerating electric field could be applied prior to or after the arrival of the argon ions at the accelerating gap.

A section view of the acceleration experiment apparatus is shown in figure 4.1.1. The ion source and modulator, including a $30 \mathrm{~cm}$ plasma drift region, were installed in a stainless steel cylinder located inside the vacuum chamber. This cylinder was held in place by a high voltage insulator, allowing the cylinder to be pulse charged to up to $100 \mathrm{kV}$ with respect to the grounded vacuum chamber. This insulator also serves as the vacuum/air interface. The ion source and modulator pulsed power supplies were connected to a 500VA UPS, all of which were located inside of a copper faraday cage. This enclosure was electrically connected to the ion source and anode assembly in the vacuum chamber. The enclosure was isolated from ground during the acceleration using a high voltage air operated relay on the 110VAC line. Triggering and diagnostic functions were carried out via fiber optic cables.

The cathode plate was electrically connected to the grounded vacuum chamber. This arrangement allowed the diagnostics used for the accelerated beam to operate at ground potential. Not shown in figure 4.1.1 are the anode and cathode electrodes which could be attached at several radial and azimuthal locations on the anode and cathode plates

At first, to achieve the acceleration, a $300 \mathrm{nF}$ capacitor was charged and then discharged through a thyratron and through the primary of an air core step-up pulse transformer. The secondary of the step-up pulse transformer was connected through a set of diodes to charge a $40 \mathrm{nF}$ capacitor. This capacitor was connected to the driver faraday cage through a $220 \Omega$ resistor. The $40 \mathrm{nF}$ capacitor was also connected to ground through $10 \mathrm{k} \Omega$ resulting in a slight droop. The voltage risetime for this system was rather slow.

Later the acceleration was accomplished using a newly designed Solid State Marx Modulator. This system used four $25 \mathrm{kV}$ stages comprised of a $25 \mathrm{kV}$ solid state switch and $25 \mathrm{nF}$ of capacitance. The figure 4.1.2 shows a comparison of the acceleration voltages created by the two systems. The fast risetime, 200ns, of the Solid State Marx Modulator based design allowed for time of flight analysis of the accelerated ion beam, the results of which are reported in a later section. The faster decay in the acceleration voltage of the Solid State Marx Modulator is due to the lower capacitance, $6.25 \mathrm{nF}$, versus the step-up transformer design, $40 \mathrm{nF}$. 


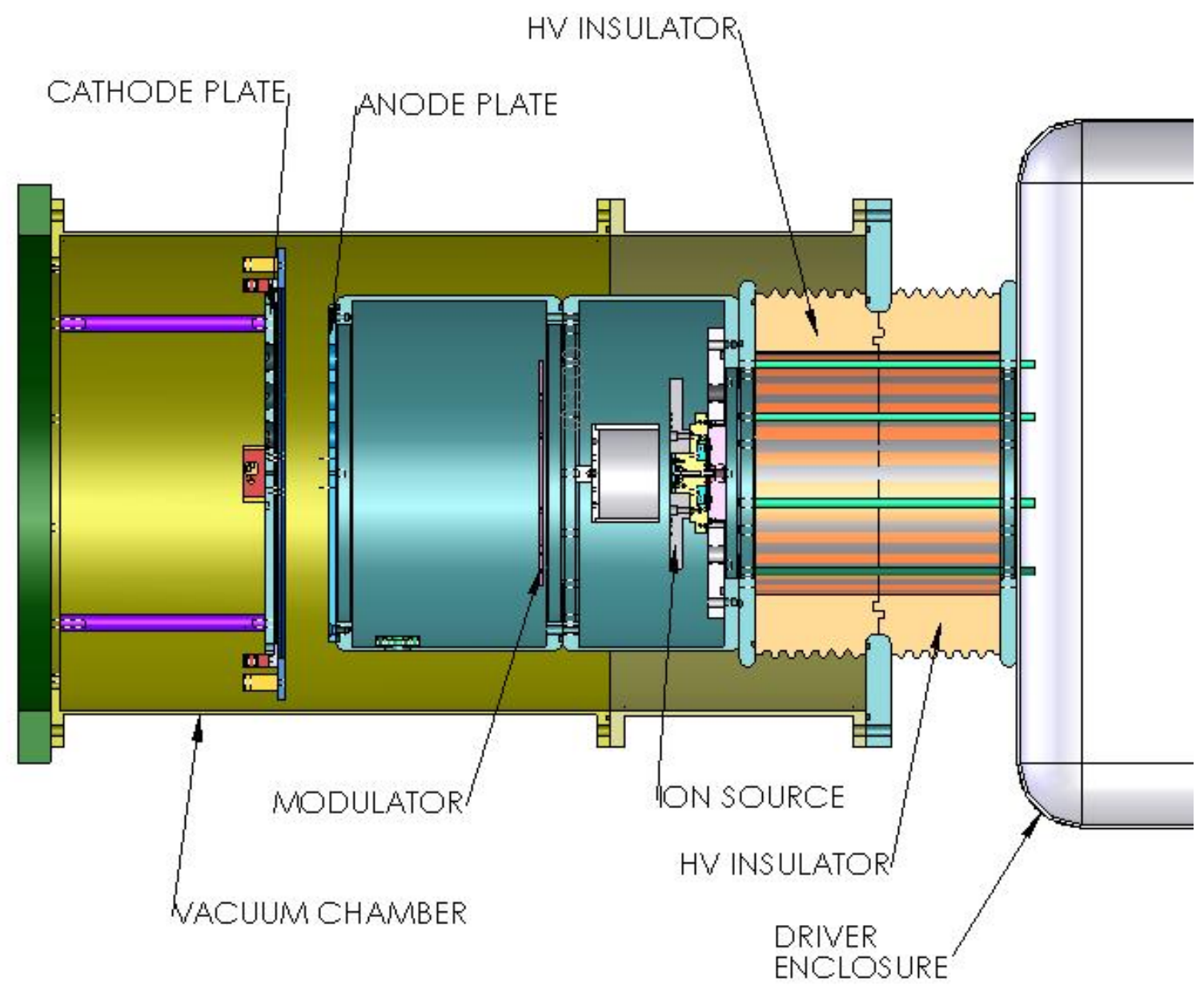

Figure 4.1.1 Section view of apparatus for ion acceleration experiments. Anode and cathode electrodes are not shown. 


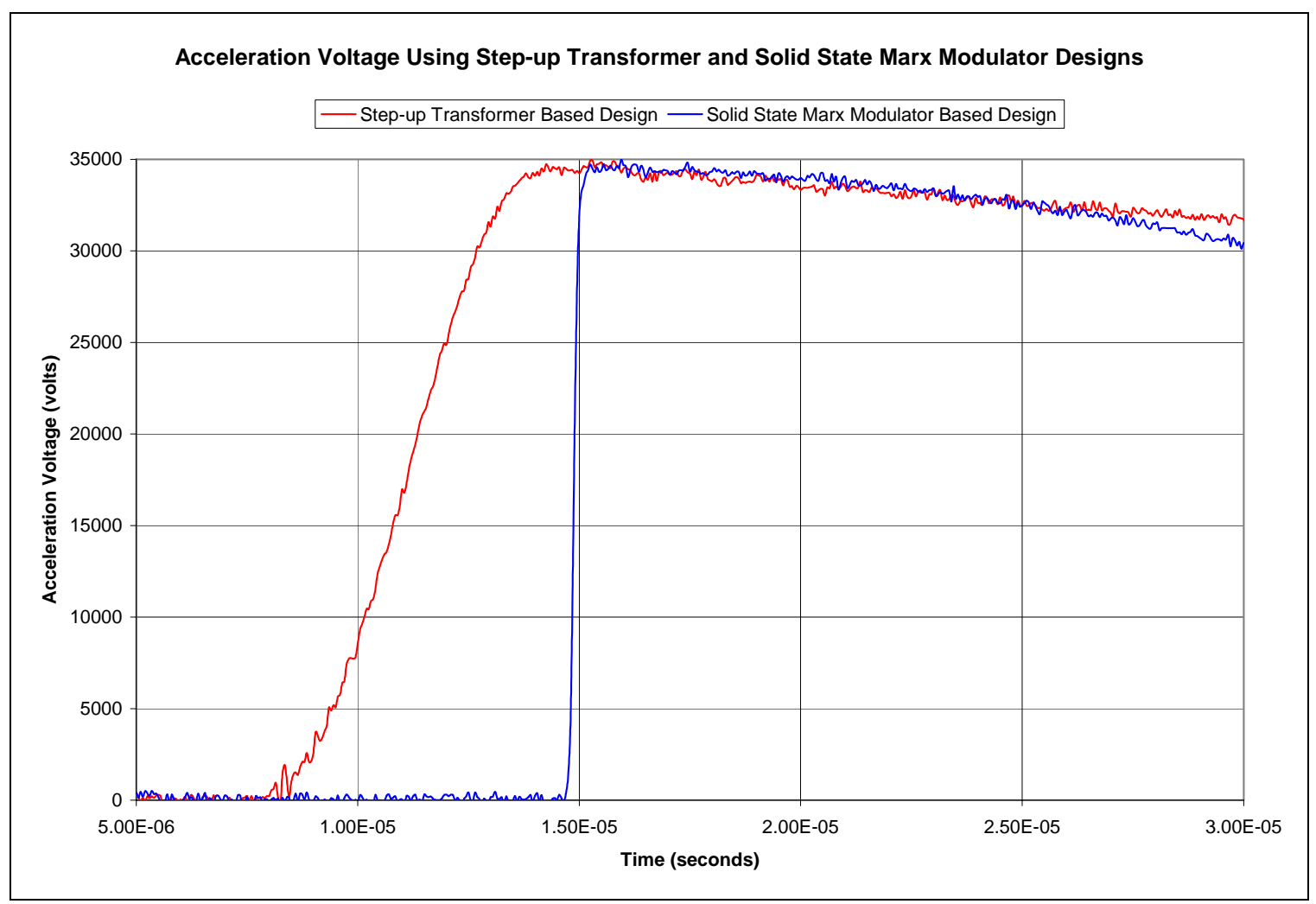

Figure 4.1.2: Comparison of Acceleration Voltage using Step-Up Transformer and Solid State Marx Modulator

Because plasma would cause the ion source to break down along surfaces in the vacuum between the drift region and the external vacuum chamber, the source was sealed to the entrance of the drift region. Vacuum pumping of the source and drift region was done through a small pumping port not directly in-line with the streaming plasma, and also through the very small hole in the anode electrode for the streaming plasma to exit. The hole in the anode electrode was, at various times, $1 \mathrm{~mm}, 1.5 \mathrm{~mm}$, or $2 \mathrm{~mm}$. The pumping port was 2" and positioned directly above the entrance to a diffusion pump. The separation between the drift region and the external vacuum chamber was several inches, except between the anode and cathode electrodes that defined the acceleration gap.

The initial design of the anode and cathode electrodes consisted of flat plates separated with an adjustable interval of $3 \mathrm{~mm}$ to $10 \mathrm{~mm}$. The edges of the plates were slightly rounded away from each other. This design proved unable to hold off more than $10 \mathrm{kV}$.

New electrodes were then designed, consisting of 3" diameter sphere segments rounded at the edge to a 2 " diameter cylinder. These electrodes were able to handle near $40 \mathrm{kV}$ at $5 \mathrm{~mm}$ separation with no plasma. However, there was a breakdown in the gap during the plasma pulse at any acceleration using the larger $1.5 \mathrm{~mm}$ and $2 \mathrm{~mm}$ apertures and above $30 \mathrm{kV}$ for the $1 \mathrm{~mm}$ aperture. This led us to purchase software (Track 6.0 from Field Precision) to calculate the electrostatic field of the gap. 
Following is the result of simulating the gap with $1 \mathrm{~mm}$ diameter apertures using a separation of $4 \mathrm{~mm}$ at $30 \mathrm{kV}$. The figure shows that this gap design resulted in a large divergence of the beam. This divergence would have resulted in a significant number of secondary electrons on the cathode which would have been accelerated towards the anode. At sufficient voltage and density, this would cause the gap to break down. The simulated result therefore correlates with the observed results.

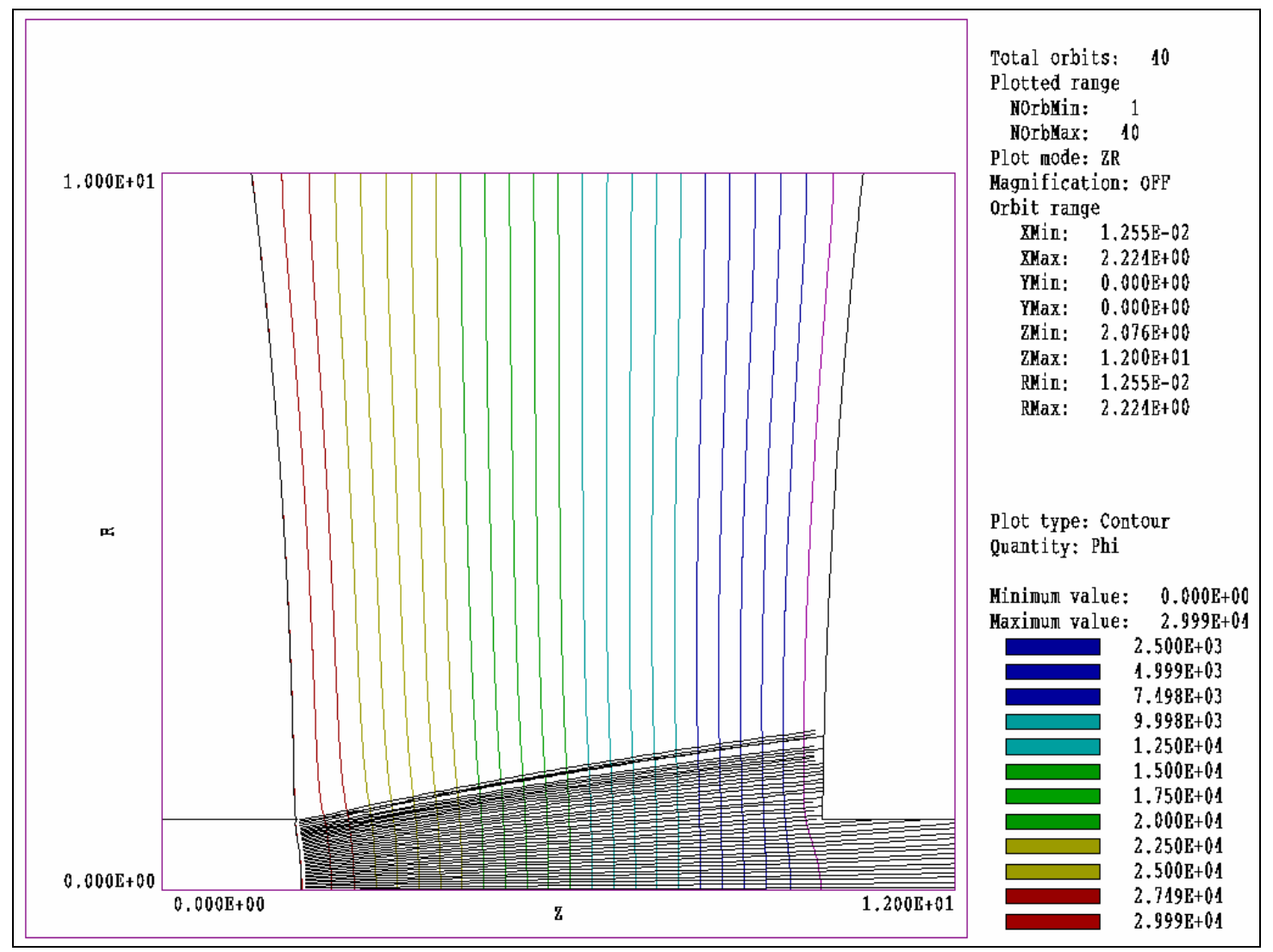

Figure 4.1.3: Ion Current Simulation for the First Design for the Acceleration Electrodes

Given this information, the electrodes were again redesigned following the design of a Pierce gun focusing electrode (see "Ion Beams with Applications to Ion Implantation" 1973). The following shows the result of simulating the newly designed gap with $1 \mathrm{~mm}$ diameter apertures using a separation of $5 \mathrm{~mm}$ at $30 \mathrm{kV}$. The angle of the anode electrode near the aperture was $67.5^{\circ}$. As can be seen in the figure, the ion beam no longer diverges in the gap, resulting in few secondary electrons being formed in it, which then allows the gap to hold off the $30 \mathrm{kV}$ during the plasma pulse. 


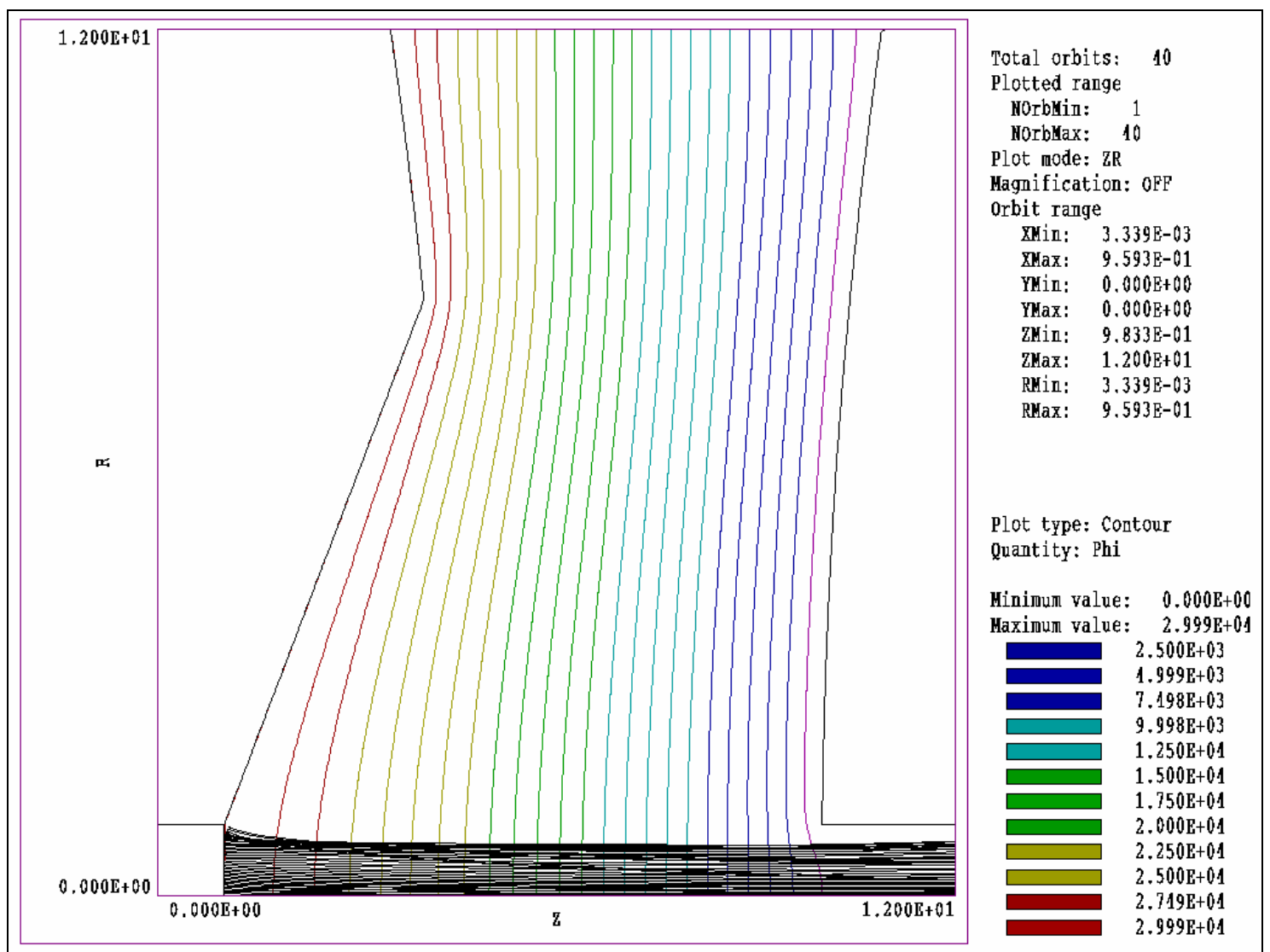

Figure 4.1.4: Ion Current Simulation for the Second Design for the Acceleration Electrodes

The Child Langmuir relationship determines the current limit for these kinds of gaps. See "Ion Sources and Injectors for the HIF Induction Linacs" Kwan, J. W., et al. Fortunately, Track 6.0 does these calculations. The CL limited current calculation for the above design was $1.2 \mathrm{~mA}$ or $150 \mathrm{~mA} / \mathrm{cm}^{2}$. The source produces a plasma pulse of up to $200 \mathrm{~mA} / \mathrm{cm}^{2}$, and so the gap was again modified to a $1.5 \mathrm{~mm}$ aperture. The CL limited current calculation for this gap was $3.6 \mathrm{~mA}$ or $200 \mathrm{~mA} / \mathrm{cm}^{2}$. This is a sufficient current to handle the maximum ion current entering the anode from the plasma.

However, this result is based on a gap separation of $5 \mathrm{~mm}$. Due to initial alignment problems, the gap broke down at closer spacing at $30 \mathrm{kV}$ with no plasma. Even at $6 \mathrm{~mm}$, the gap broke down at around $40 \mathrm{kV}$ with no plasma. Also, a small change from $5 \mathrm{~mm}$ to $6 \mathrm{~mm}$ would result in a $30 \%$ reduction in the CL current limit as the current is proportional to $1 / \mathrm{d}^{2}$. Additional work was required to improve the alignment of the electrodes. 


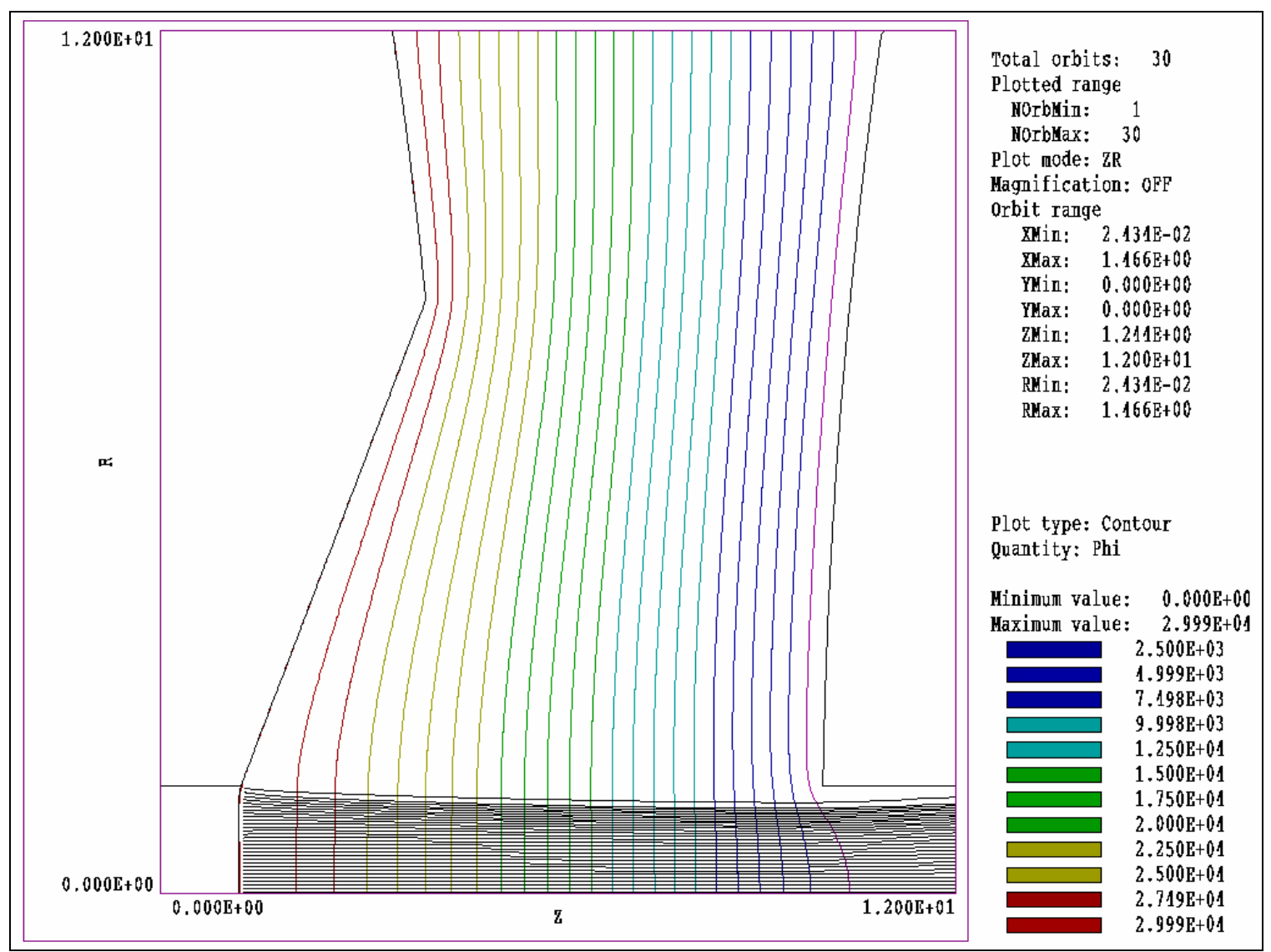

Figure 4.1.5: Ion Current Simulation for the Third Design for the Acceleration Electrodes

Initial examination of the accelerated ion beam was done by viewing the luminescence of a phosphor screen illuminated by the accelerated ion beam. The ICCD camera used previously was again employed to capture the images of the phosphor screen, placed at a $45^{\circ}$ angle to the incident beam. The camera was located at a view port at $90^{\circ}$ to the incident beam. Figure 4.1.6 shows an example picture. This data was from a $2 \mathrm{~mm}$ aperture in the cathode acceleration electrode with a distance of $79 \mathrm{~mm}$ from the cathode electrode to the phosphor screen.

The shape of the spot is elongated in the horizontal directions due to the angle of the phosphor screen to the ion beam, and also distorted by the edges of the phosphor screen. Additionally, the outline of the metal clamp holding the phosphor screen at the edge can be observed in the lower right of the spot. Little light was produced by this area because the phosphor was covered by the clamp. The data was then fit to produce horizontal and vertical widths for the spot as shown in Figure 4.1.7 and 4.1.8. 


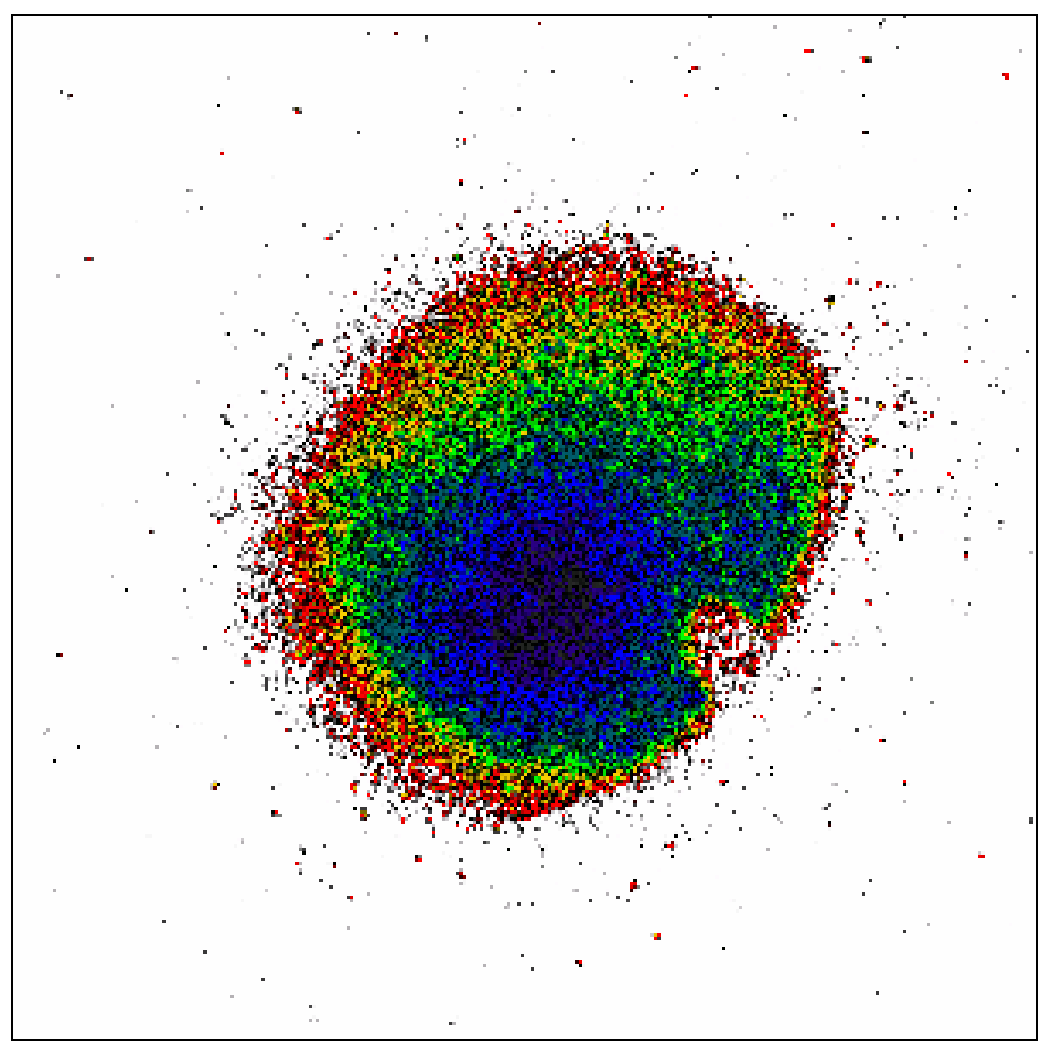

Figure 4.1.6: Picture of Luminescence of Phosphor Screen Illuminated by Accelerated Ion Beam

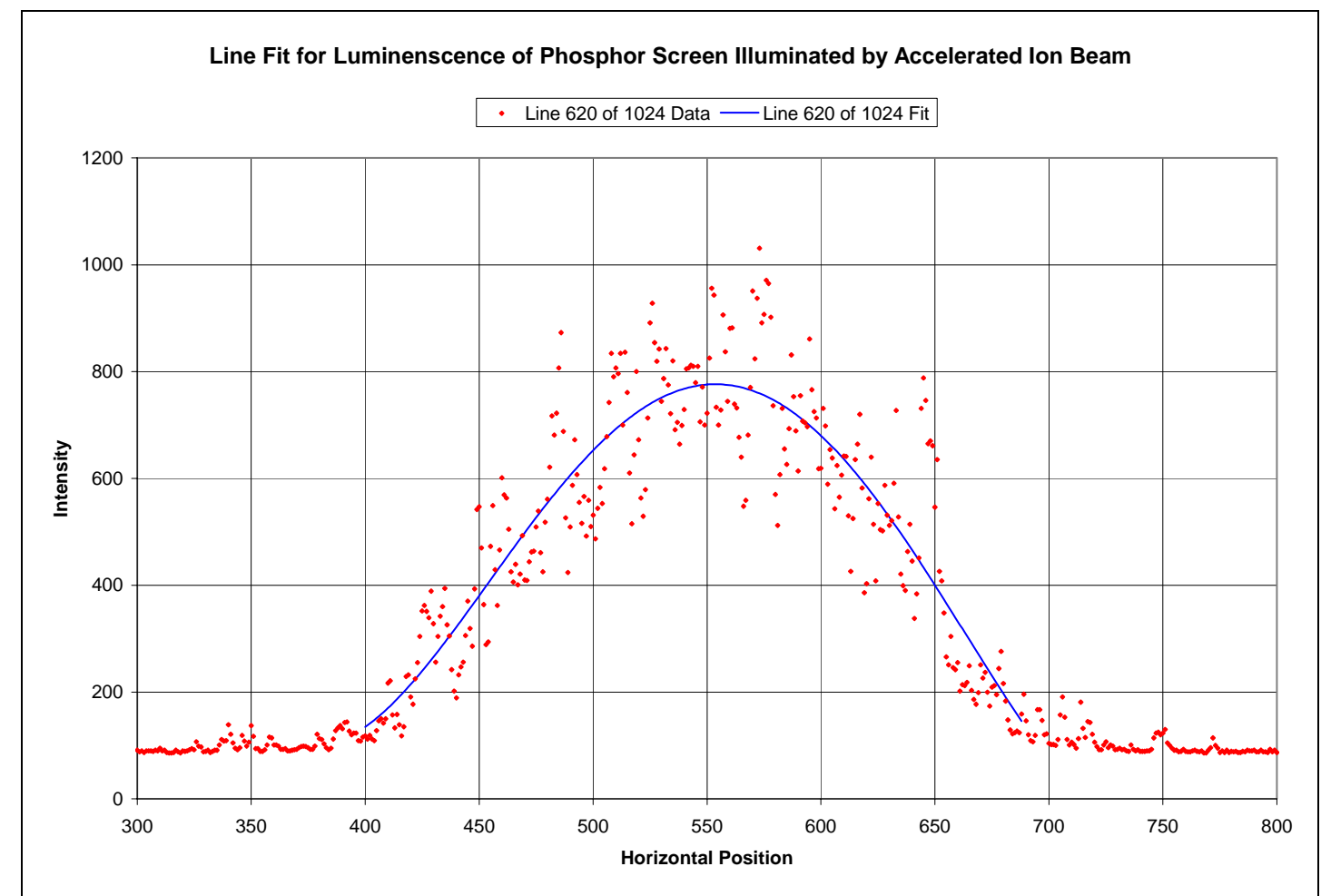

Figure 4.1.7: Horizontal Line Fit of Luminescence of Phosphor Screen Illuminated by Accelerated Ion Beam 


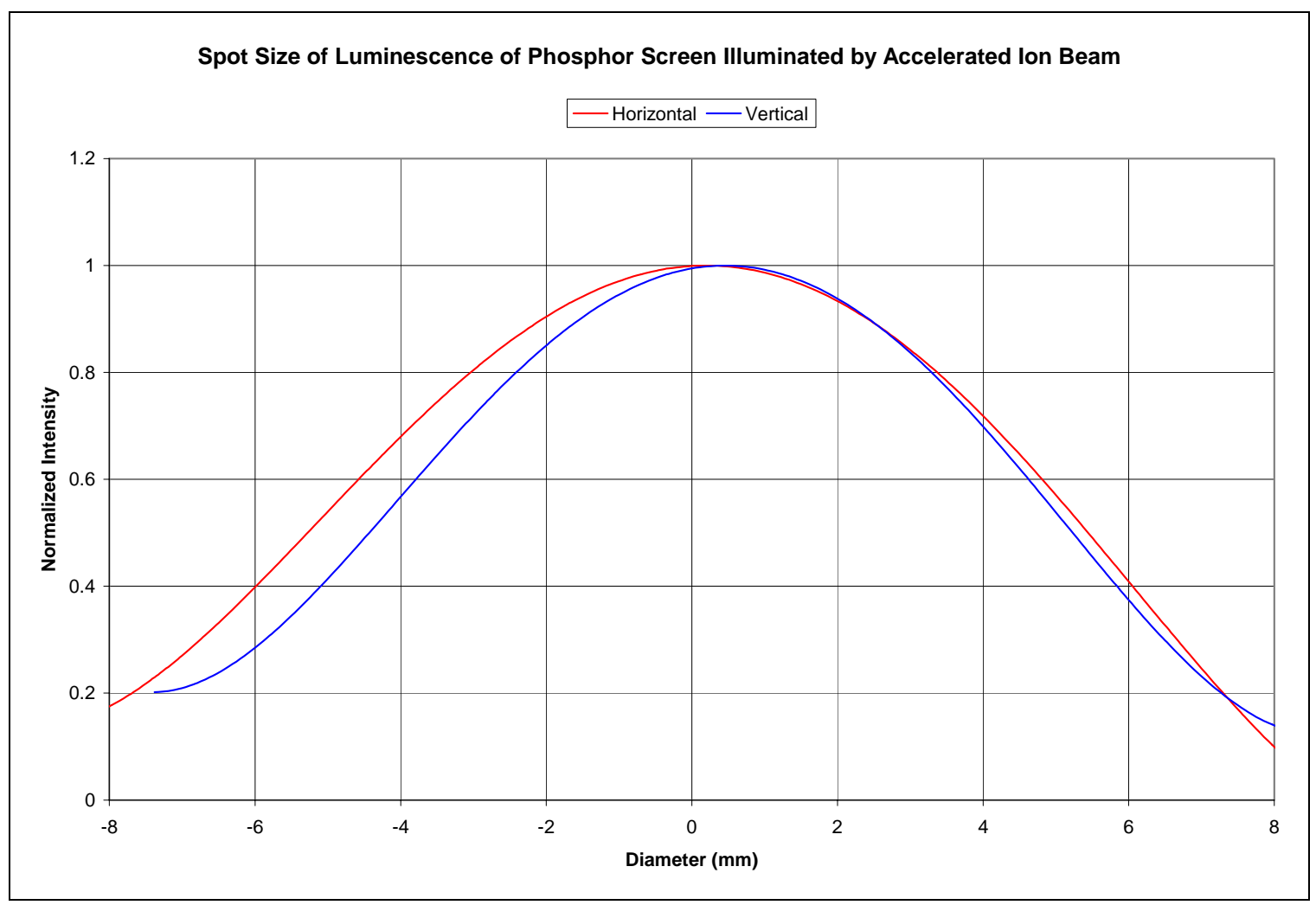

Figure 4.1.8: Spot Size of Luminescence of Phosphor Screen Illuminated by Accelerated Ion Beam

This shows the horizontal broadening due to the angle of the phosphor screen. If we take the 0.2 level as the width of the beam, then the beam expanded from $2 \mathrm{~mm}$ to $14 \mathrm{~mm}$ over $79 \mathrm{~mm}$. That would be an angle of divergence of about $3.62^{\circ}$ for the accelerated beam. Figure 4.1.9 shows the simulated beam divergence from the cathode electrode. The simulation shows the accelerated ion beam expanding to $7 \mathrm{~mm}$ radius, $14 \mathrm{~mm}$ diameter, at $80 \mathrm{~mm}$. That corresponds to the spot size seen on the phosphor screen. So the observed divergence of the accelerated ion beam can be attributed to the design of the acceleration electrodes and the drift region between the cathode electrode and the phosphor screen. 


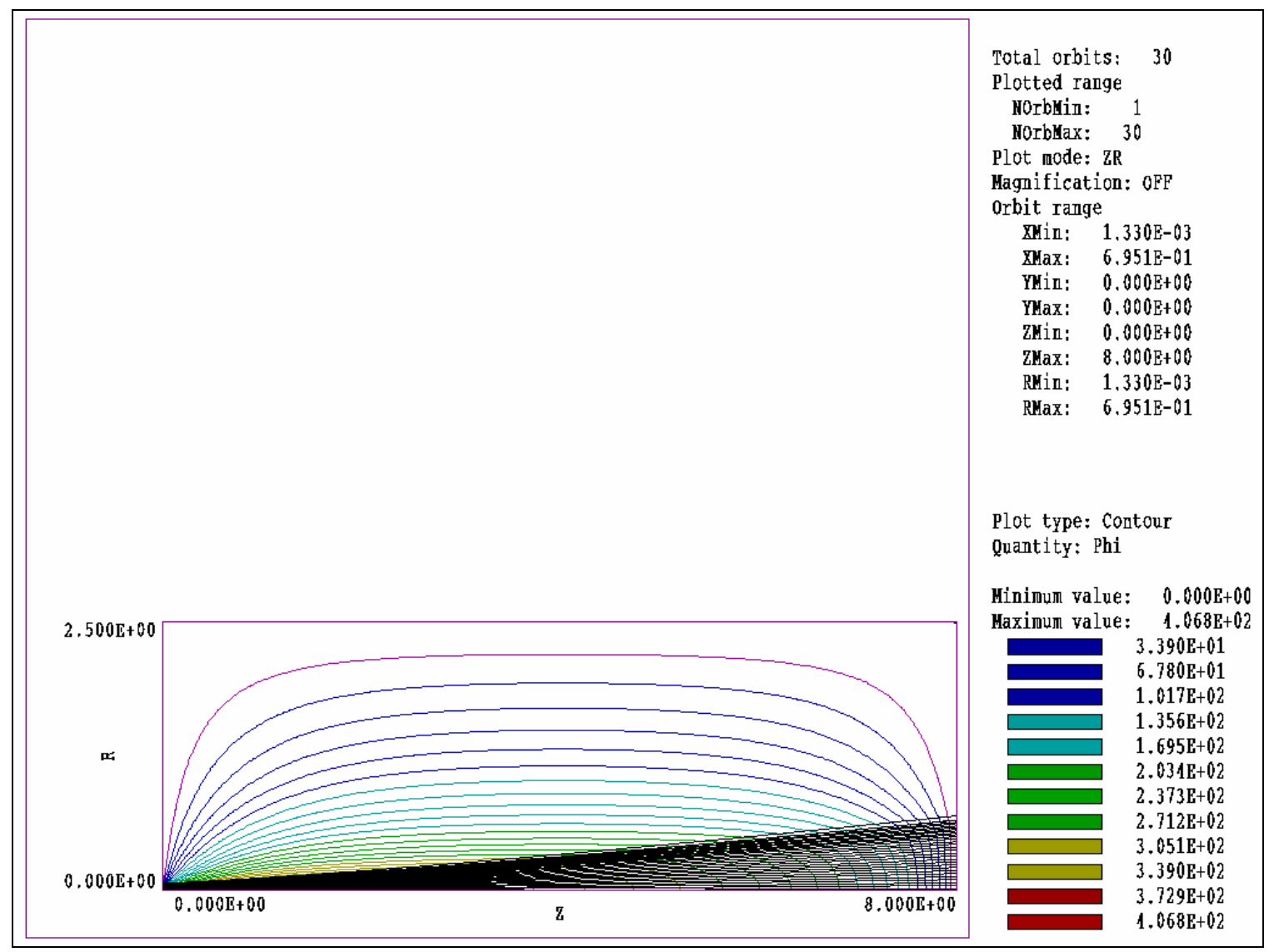

Figure 4.1.9: Simulated Divergence of Accelerated Ion Beam 


\subsection{Acceleration and Modulation}

\subsubsection{Acceleration and Modulation before the Changes in the Gap}

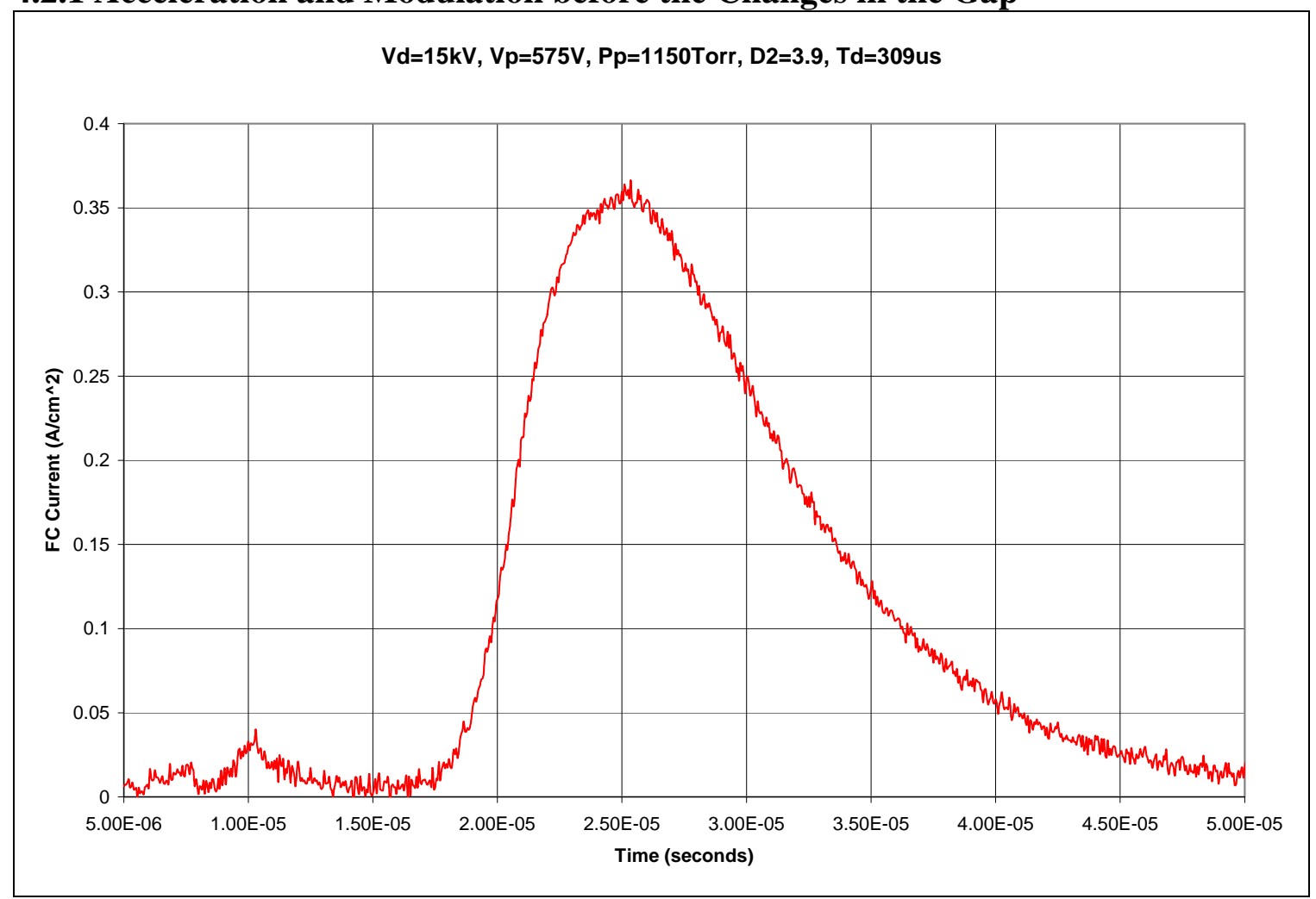

Figure 4.2.1.1: Modulated Ion Current without Acceleration

The modulator is designed to produce a flatter top to the ion beam. Initial tests were performed with the ion beam without acceleration and without the anode and cathode gap. This allows the full ion beam to travel from the modulator to the $1 / 8^{\text {th }}$ inch hole in front of the Faraday cup. The above graph shows an unmodified ion beam current as measured by the FC. As can be seen, it does not have a significantly long flat top. The following graph shows the effect of timing of the modulator current on the ion beam density. 


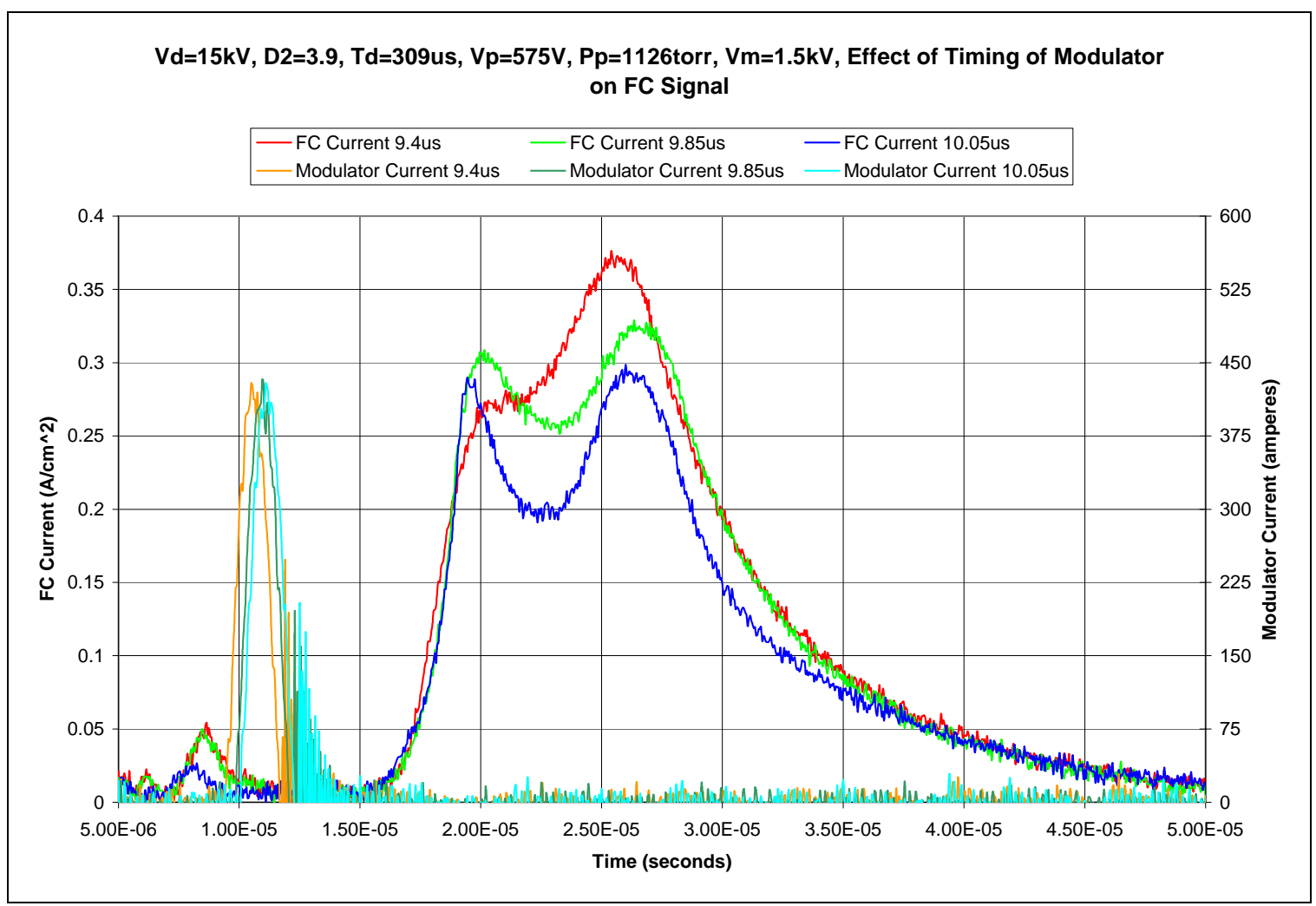

Figure 4.2.1.2: Effects of Short Period Modulator Current Timing on the Ion Current without Acceleration

As can be seen in the above graph, a small shift in the timing of the modulator current alters the shape of the FC signal. Also, the fact that such a small width of the FC signal is being modulated, indicates that the modulator current pulse shape is not wide enough to create a flat top on the FC signal.

Additional inductance was placed in series with the modulator coil. This resulted in a wider modulator current pulse. The effect of timing using this wider pulse can be seen in the following graph. 


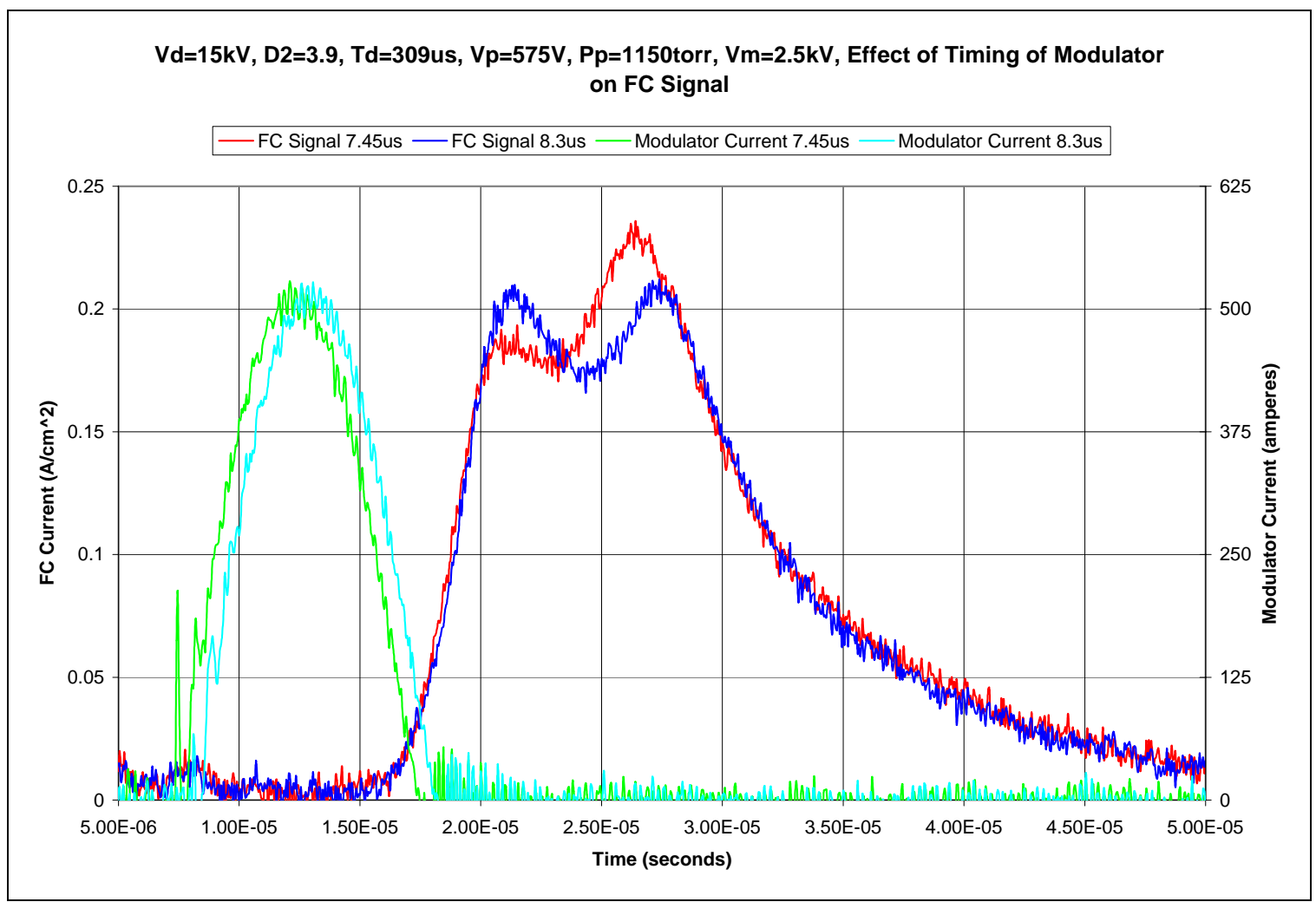

Figure 4.2.1.3: Effects of Long Period Modulator Current Timing on the Ion Current without Acceleration

It should be noted that the magnitude of the FC current at the center of the pulse has not changed from the previous setup with the thinner modulator current pulse. However, the peak to trough from $20 \mu \mathrm{s}$ to $29 \mu \mathrm{s}$ is from $173 \mathrm{~mA} / \mathrm{cm}^{2}$ to $210 \mathrm{~mA} / \mathrm{cm}^{2}$. Previously the peak to trough from $19 \mu \mathrm{s}$ to $29 \mu \mathrm{s}$ was from $190 \mathrm{~mA} / \mathrm{cm}^{2}$ to $300 \mathrm{~mA} / \mathrm{cm}^{2}$ or from $250 \mathrm{~mA} / \mathrm{cm}^{2}$ to $330 \mathrm{~mA} / \mathrm{cm}^{2}$. This is an improvement from $\pm 22 \%$ or $\pm 14 \%$ to better than $\pm 10 \%$ over a similar time period.

The results above were obtained using $2.5 \mathrm{kV}$ for the charge on the modulator power supply. This produced a peak current in the modulator coils of just over 500A. The previous setup used a $1.5 \mathrm{kV}$ charge and obtained less than a $450 \mathrm{~A}$ current. The following tests were performed using the new setup with a $2 \mathrm{kV}$ charge. In this case, the current was again less than 450A. This yielded a FC current peak of $200 \mathrm{~mA} / \mathrm{cm}^{2}$ to $300 \mathrm{~mA} / \mathrm{cm}^{2}$, which is only slightly better flatness than the earlier results over the same time period. 


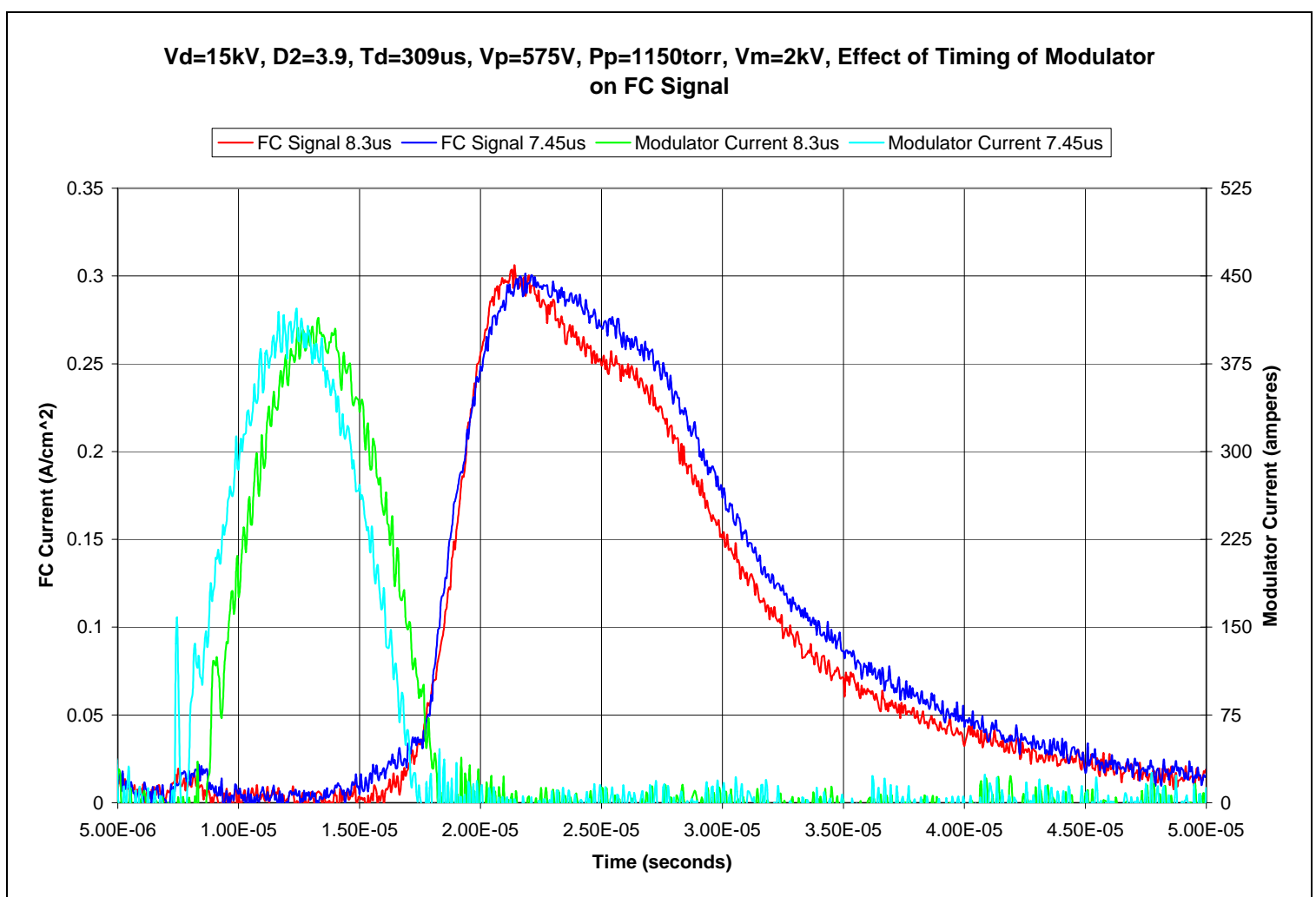

Figure 4.2.1.4: Effects of Lower Peak Current Long Period Modulator Current Timing on the Ion Current without Acceleration

Finally, the anode and cathode gap was reinstalled and acceleration of $35 \mathrm{kV}$ was used. The anode and cathode gaps are $2 \mathrm{~mm}$, resulting in a smaller angular acceptance angle for the FC. Also, there is a space charge limit to the ion beam current emitted by the anode gap, which also reduces the ion beam current detected by the FC. Figure 4.2.1.5 shows the FC signal when the gap broke down during the ion beam current pulse. The modulator was not used in the test in which the gap broke down, so there was a higher peak ion beam current, the probable reason for the gap breakdown, because the peak current exceeded the maximum space charge limit the gap could transport. It is seen that after the gap voltage collapsed, the rest of the ion beam current passed unhindered through the gap and into the FC, producing a second, later peak.

If the ion current density supplied to the anode is higher than the space-charge-limited current density that can be transported across the gap at a given accelerating voltage, then charge builds up at the anode and begins to fill the gap. This distorts the gap electric field, spreading the ion trajectories across the gap so that the cathode is bombarded, thus eventually resulting in breakdown. For optimal performance of the accelerating gap to extract the highest possible current density at the lowest possible divergence, the supplied ion current density in the plasma streaming into the anode must equal the extracted current density at all positions across the radius of the anode entrance hole, thereby allowing the formation of a quasi-stationary plasma surface from which the ions are extracted. 


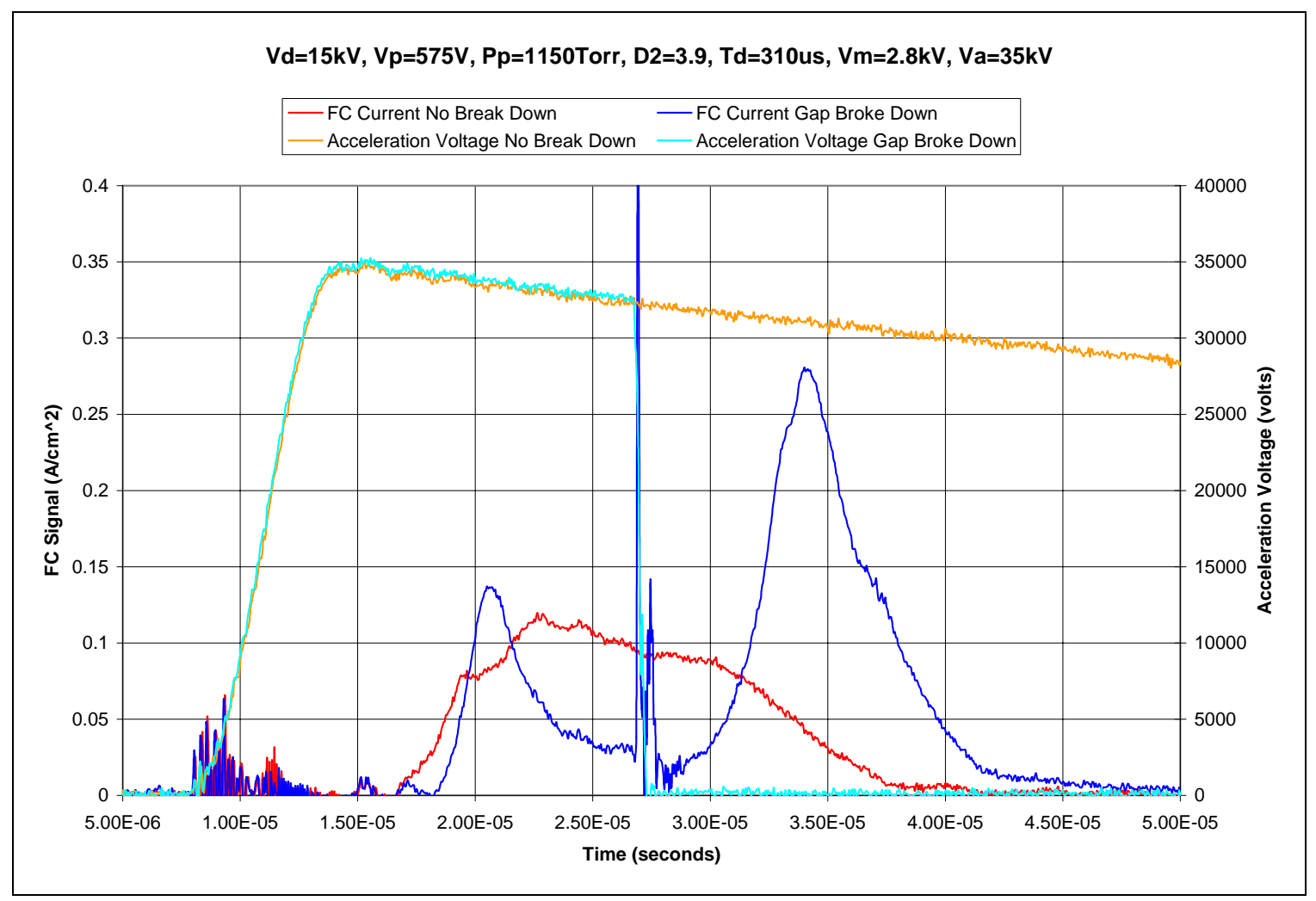

Figure 4.2.1.5: Ion Current with Modulation and Acceleration with and without a Breakdown in the Acceleration Gap

The accelerated pulse, when the modulator is used, results in a much lower peak ion beam current. However, the goal was to exceed $100 \mathrm{~mA} / \mathrm{cm}^{2}$, achieved with these signals. Here, the peak signal is from $19 \mu \mathrm{s}$ to $31 \mu \mathrm{s}$, with a peak to trough of $100 \mathrm{~mA} / \mathrm{cm}^{2}$ to $130 \mathrm{~mA} / \mathrm{cm}^{2}$. This would be $\pm 13 \%$, or again slightly better than the earlier results but for a slightly longer time. Some of this drop may be associated with the droop in the acceleration voltage, which over $10 \mu$ s is about $10 \%$.

So far, this data indicates that an accelerated beam can be made with a peak current of over $100 \mathrm{~mA} / \mathrm{cm}^{2}$ for over $10 \mu \mathrm{s}$. It was this beam that was used for further testing, with a gate composed of an electric field deflector as well as a phosphor screen. 


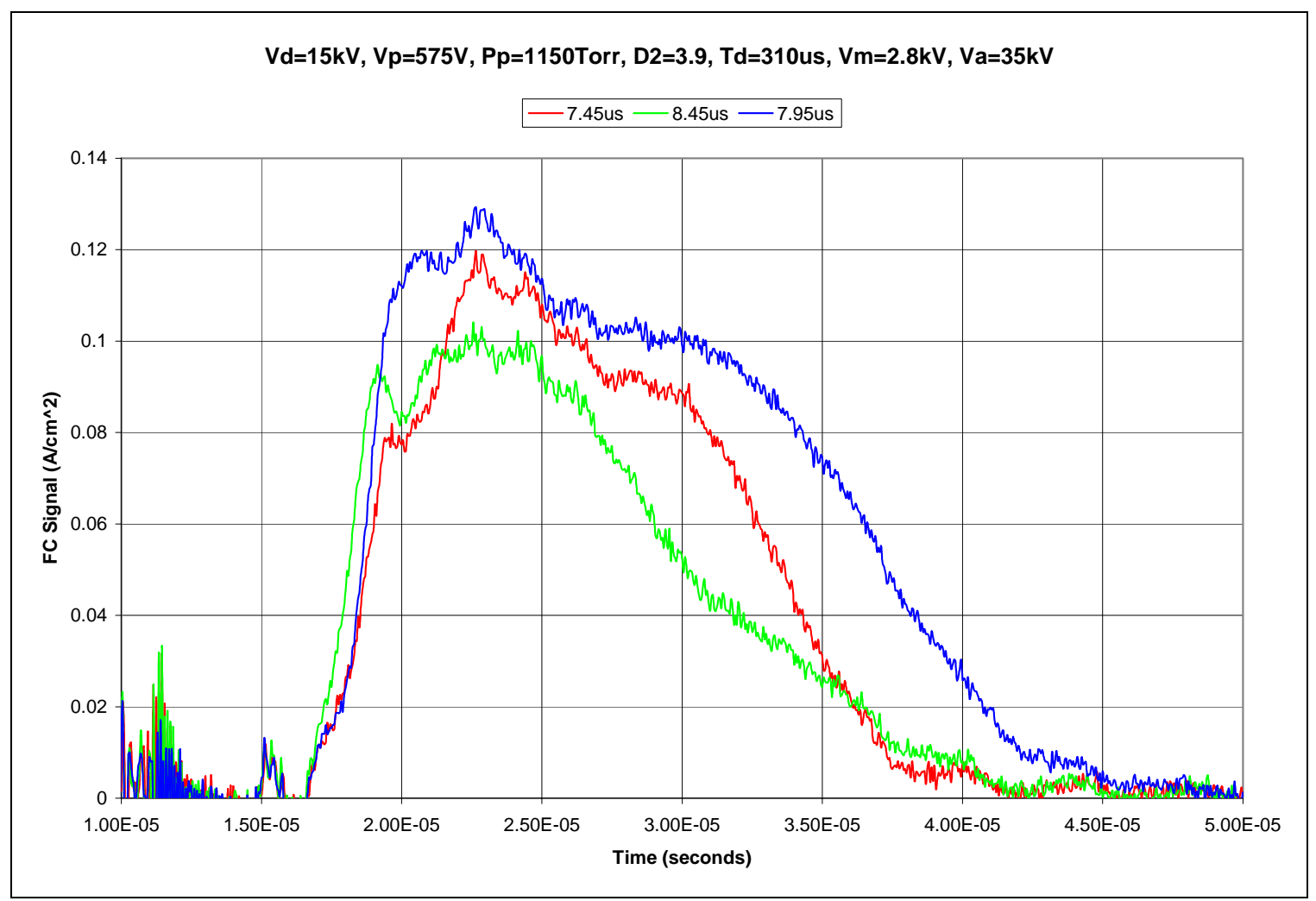

Figure 4.2.1.6: Effects of Modulator Timing on Accelerated Ion Current

\subsubsection{Time of Flight Analysis of Accelerated and Modulated Ion Beam Using Final Design}

Analysis of the accelerated ion beam for time of flight was used to examine the species existing in the ion beam. Following is a typical ToF (time of flight) data set. 
Applied Pulsed Power, Inc.

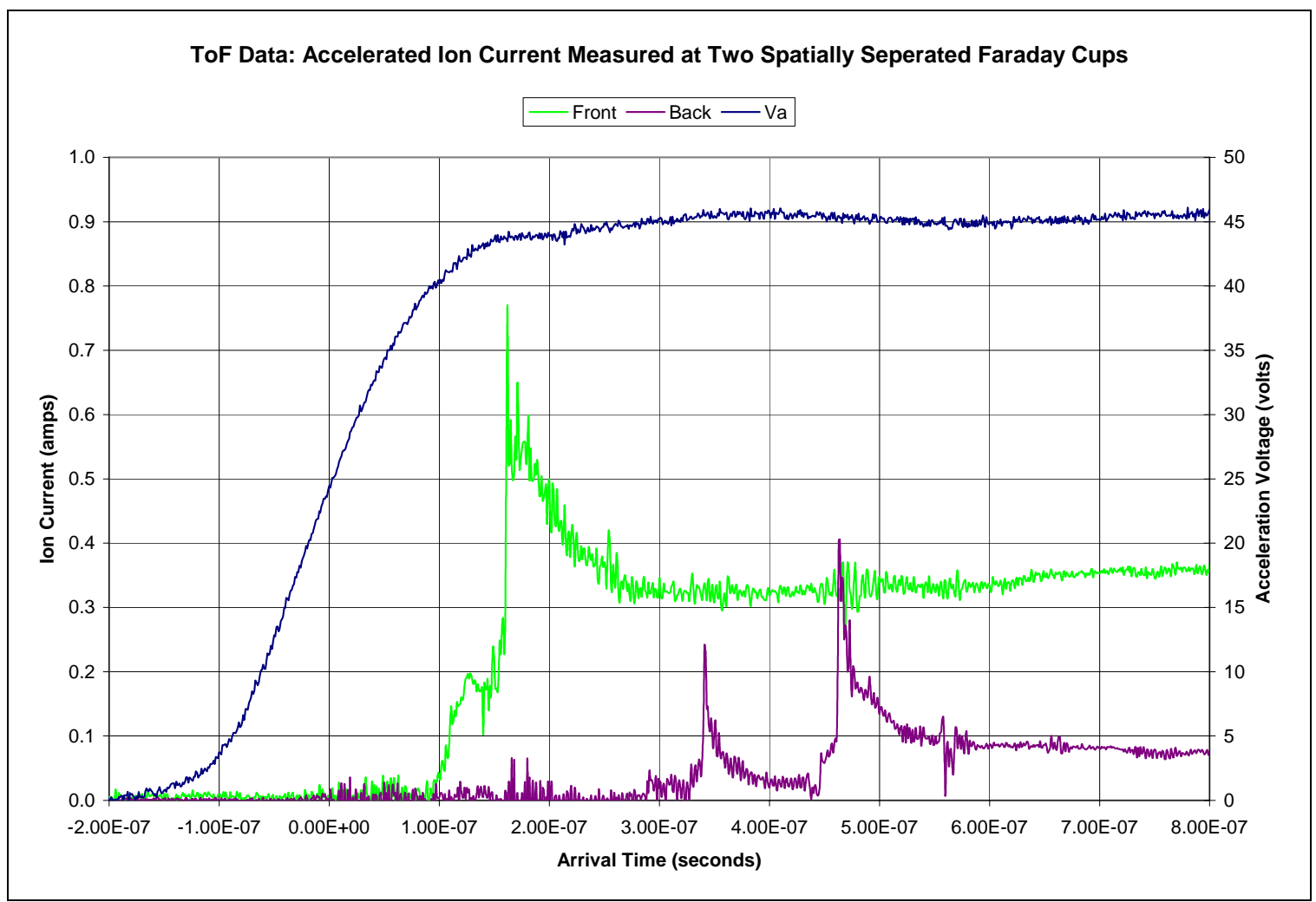

Figure 4.2.2.1: Faraday Cup Current at Two Different Z locations

The acceleration voltage waveform was used to predict the arrival times for $\mathrm{Ar}^{1+}$ and $\mathrm{Ar}^{2+}$ as seen in the next graph. 


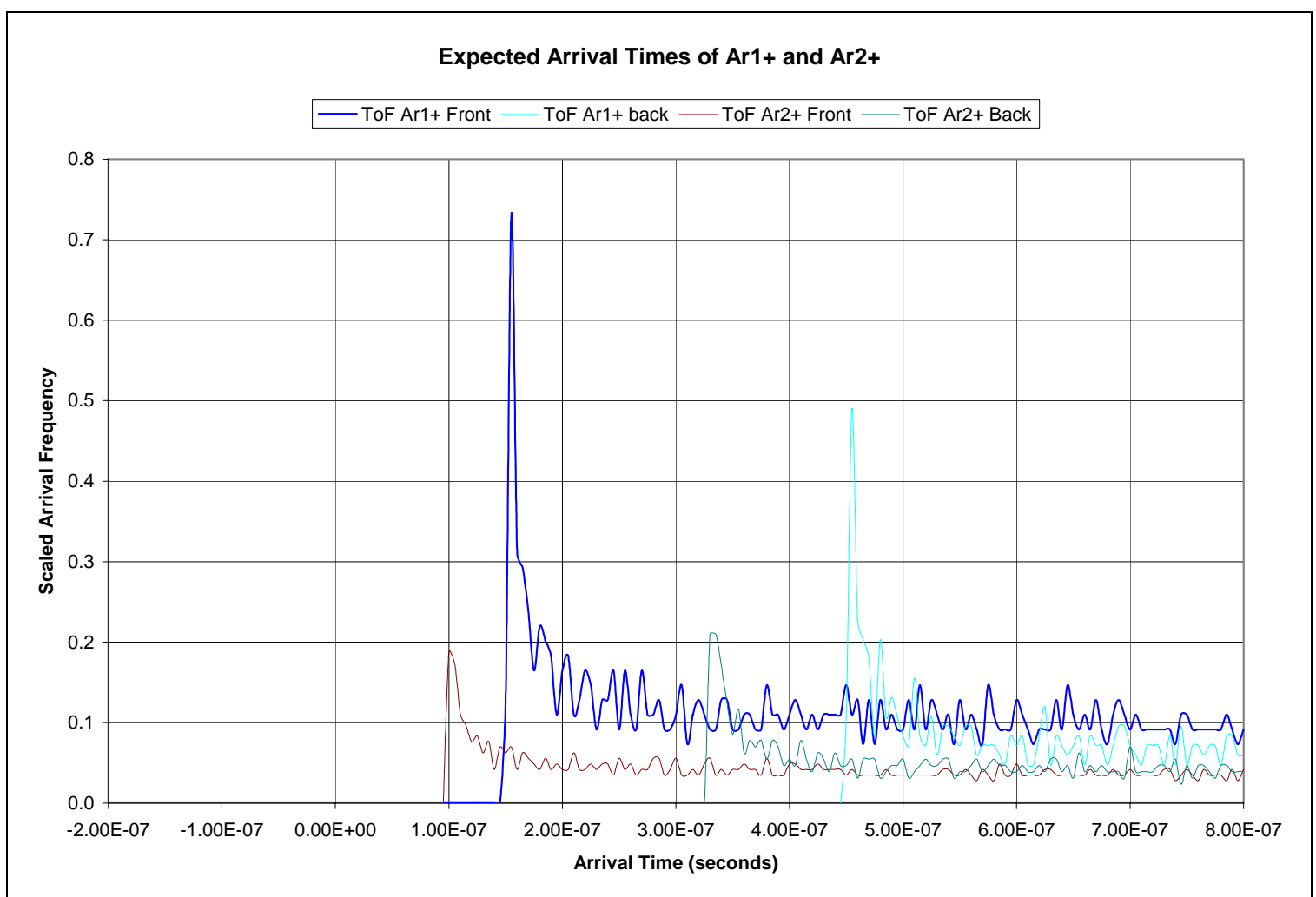

Figure 4.2.2.2: Calculated Arrival Times Given Known Acceleration Profile for $\mathrm{Ar}^{1+}$ and

$$
\mathrm{Ar}^{2+}
$$

This graph clearly demonstrates that $\mathrm{Ar}^{1+}$ and $\mathrm{Ar}^{2+}$ both exist in the accelerated ion beam at a ratio of about 8:1. This does not correlate with the spectrographic data as previously reported. While that data did clearly show the existence of $\mathrm{H}, \mathrm{C}, \mathrm{N}$, and $\mathrm{O}$ in the plasma, there was no trace of $\mathrm{Ar}^{2+}$ above the noise level of the detector.

The acceleration voltage waveform was then used to analyze for $\mathrm{H}, \mathrm{C}, \mathrm{N}$, and $\mathrm{O}$. The $\mathrm{H}$ peak at the forward faraday cup was at $-0.03 \mu$ s and at $0.055 \mu$ s at the rear FC. For C, the times were $0.07 \mu \mathrm{s}$ and $0.26 \mu \mathrm{s}$. For $\mathrm{N}$ the times were $0.08 \mu \mathrm{s}$ and $0.29 \mu \mathrm{s}$. Finally, for $\mathrm{O}$ the arrival times were $0.09 \mu \mathrm{s}$ and $0.305 \mu \mathrm{s}$. The ToF data does show small peaks around these times, indicating that these elements existed in the accelerated ion beam at ratios less than 1:50 to the $\mathrm{Ar}^{1+}$ ion current.

Further TOF investigation of variations with the plasma driver voltage is shown in Figure 4.2.2.3. These data show that both $\mathrm{Ar}^{1+}$ and $\mathrm{Ar}^{2+}$ ion currents are strongly dependent on the plasma driver voltage, and that the $\mathrm{Ar}^{2+}$ ion current is nearly proportional to the $\mathrm{Ar}^{1+}$ ion current. If the second ionization were taking place in the source, it might be expected that the proportion of $\mathrm{Ar}^{2+}$ to $\mathrm{Ar}^{1+}$ would be strongly dependent on source voltage, while if the second ionization is taking place in the accelerating gap, the proportion of the two charge states should not vary with source voltage. Thus this data supports the inference that the $\mathrm{Ar}^{2+}$ is being produced in the accelerating gap. 
While the plasma source driver creates electric fields on the order of $100 \mathrm{~V} / \mathrm{cm}$, the acceleration gap has fields on the order of $100,000 \mathrm{~V} / \mathrm{cm}$. So the acceleration gap has three orders of magnitude greater electric field strength as well as higher voltage. Electrons from the cathode, produced as secondaries by bombarding ions, would be very effective ionizers of the $\mathrm{Ar}^{1+}$ ions crossing the gap. Optimized electrode design, along with control of the supplied ion current density, would minimize the production of backstreaming electrons and reduce the $\mathrm{Ar}^{2+}$ production.

The ToF data can also in principle be used to measure the energy spread of a given ion species in the beam. As seen in the figures, the finite risetime of the accelerating voltage results in higher-energy ions accelerated later in the rise catching up to lower energy ions accelerated earlier. The result is the initial peak of ion current in the Faraday cups. This effect makes quantitative unfolding of the amplitude of the Faraday cup signals with time quite insensitive to a small energy spread in the beam, and the results show only that the spread is undetectable, below a few percent.

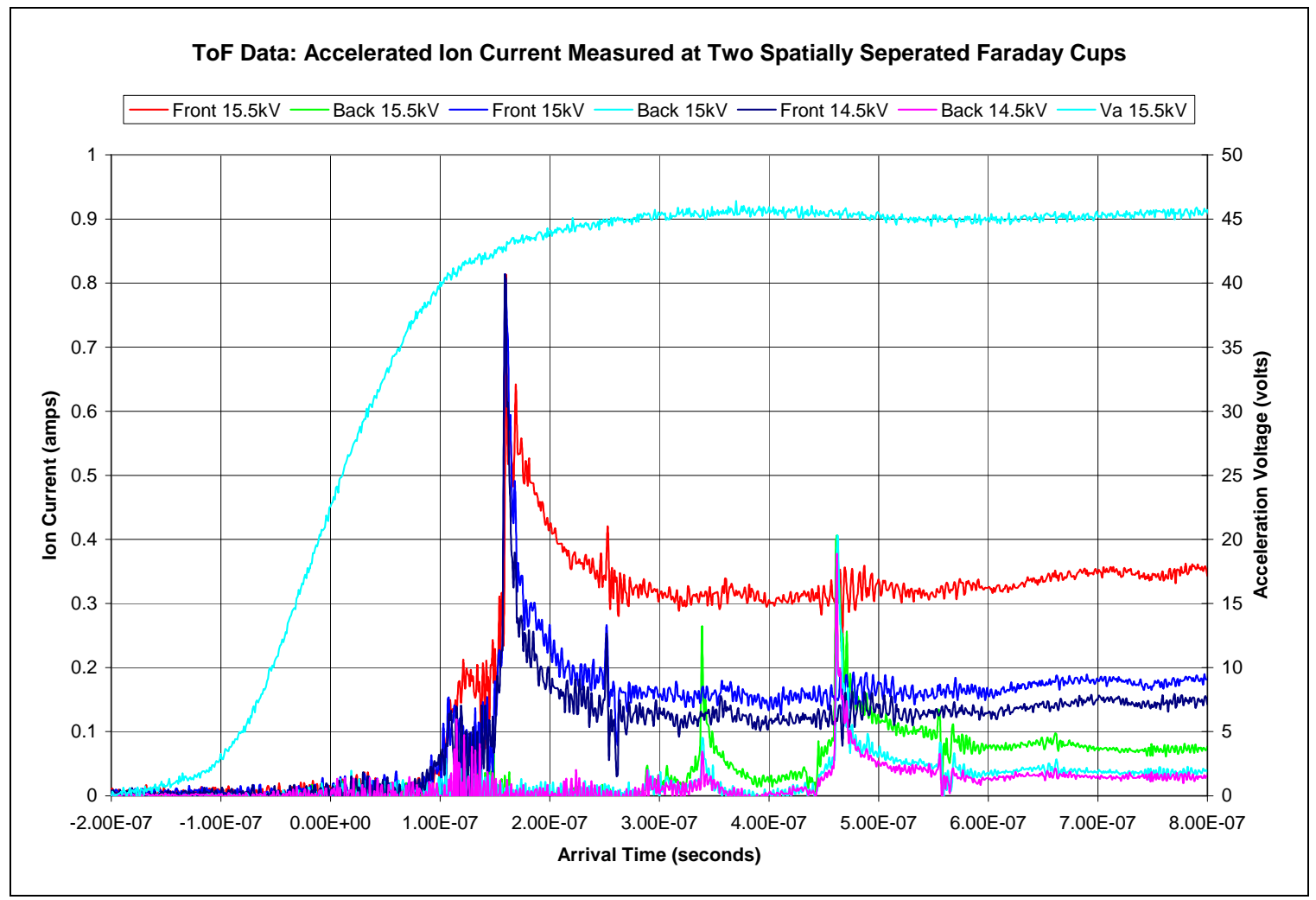

Figure 4.2.2.3: Faraday Cup Current at Two Different Z locations for Different Source Plasma Driver Charge Voltages 


\subsubsection{Pepper pot data}

A "pepper pot" diagnostic was employed to measure aiming and divergence of the accelerated beam. This pepper pot consisted of an aperture plate with an array of small holes, followed by a target to record the distribution of ions passing through the holes. The target was the phosphor screen (used in the data for Figure 4.1.6 above). Figure 4.3.1.1 shows contour plots of the image from an accelerated beam in the pepper pot, and Figure 4.3.1.2 shows line scans of the intensity of the spots. Analysis of this data shows two things. First, the full width of each of the spots is less than or equal to $2 \mathrm{~mm}$. This is actually slightly less than the width of $2.6 \mathrm{~mm}$ expected if each of the spots is an image of the source with perfect straight-line ion trajectories. Any of three possible causes might be responsible.

First, as the simulations (eg Figure 4.1.5) show, the beam may not entirely fill the gap aperture, making the effective source smaller than the full $2 \mathrm{~mm}$ electrode exit diameter. Second, if the beam is well charge-neutralized but not entirely current-neutralized, some pinching could be occurring, decreasing the radius. Third, some erosion of the edges of the beamlets that pass the pepper pot apertures could be occurring, making them smaller at the target. There is no direct evidence that any of these effects are occurring.

Second, the spacing between the centers of the pepper pot spots is also consistent (5.0 $\mathrm{mm}+-0.3 \mathrm{~mm}$ in the vertical direction) with the expected spacing for straight-line ion trajectories from the gap (4.96 $\mathrm{mm}$ spacing).

The only conclusion that can be drawn from these data is that any deviation of the beam optics from straight-line trajectories is smaller than the possible competing effects listed above, and the overall divergence is consistent with expectation from the simulations of section 4.1. There is no increase in the transverse ion velocity observable due to source temperature. With this diagnostic, a $0.5 \mathrm{~mm}$ spread of the beam would be about at the threshold of sensitivity. This corresponds to about $4 \mathrm{eV}$ transverse temperature at the source, so the source must be better than this. This is however nearly an order of magnitude above the $0.6 \mathrm{eV}$ measured in the source itself, so the results do not rule out that level of emittance increase in the acceleration process.

These results therefore are not sufficiently sensitive to allow quantitative evaluation of the transverse source temperature, but only to give an upper limit. Clearly, the beam quality is very high, and could be quantitatively measured only with more sophisticated beam extraction and transport experiments in a real accelerator, which is beyond the scope of the present program. 


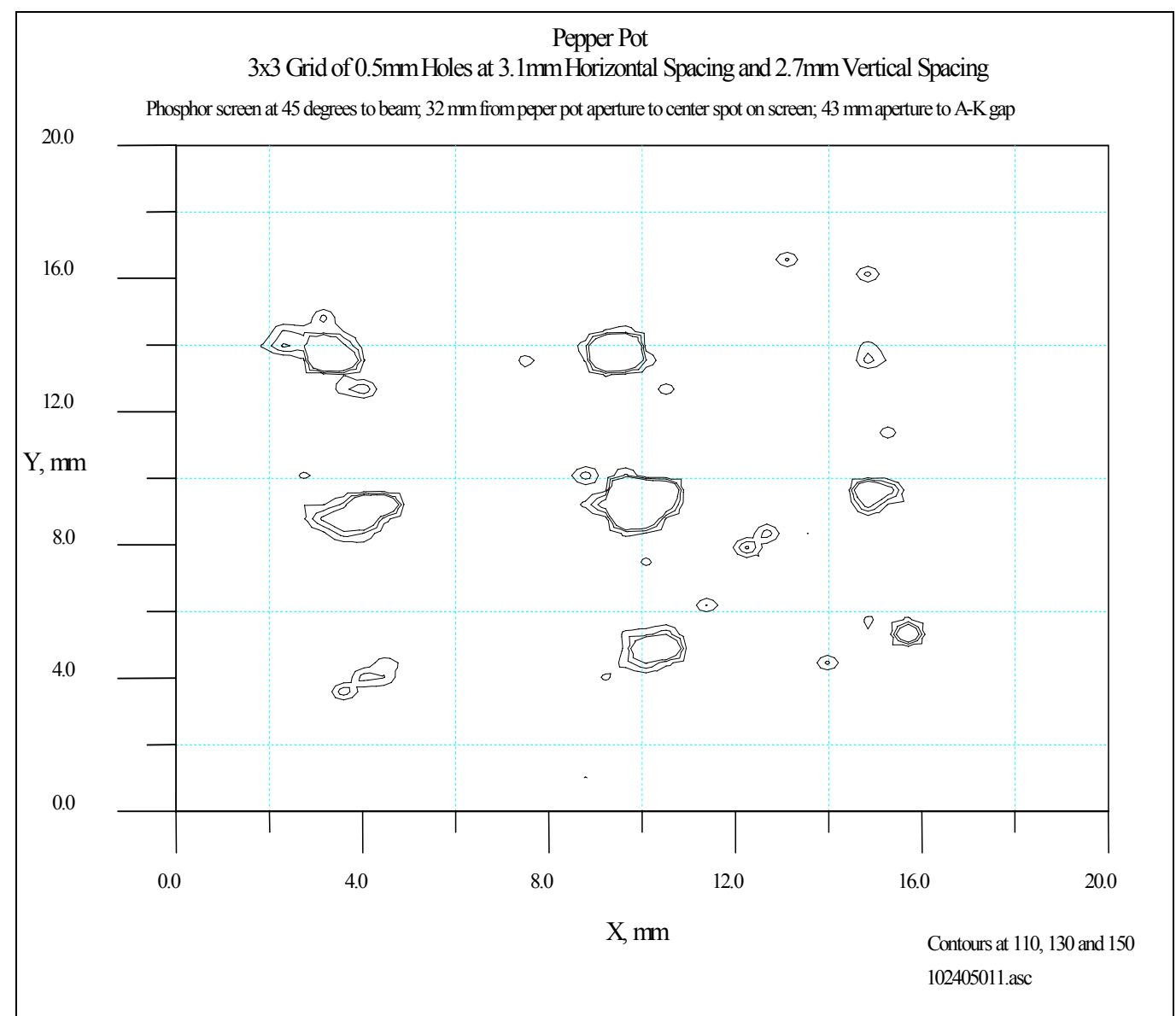

Figure 4.2.3.1: Pepper pot data. Contour plots from digital image of fluorescent screen target. 


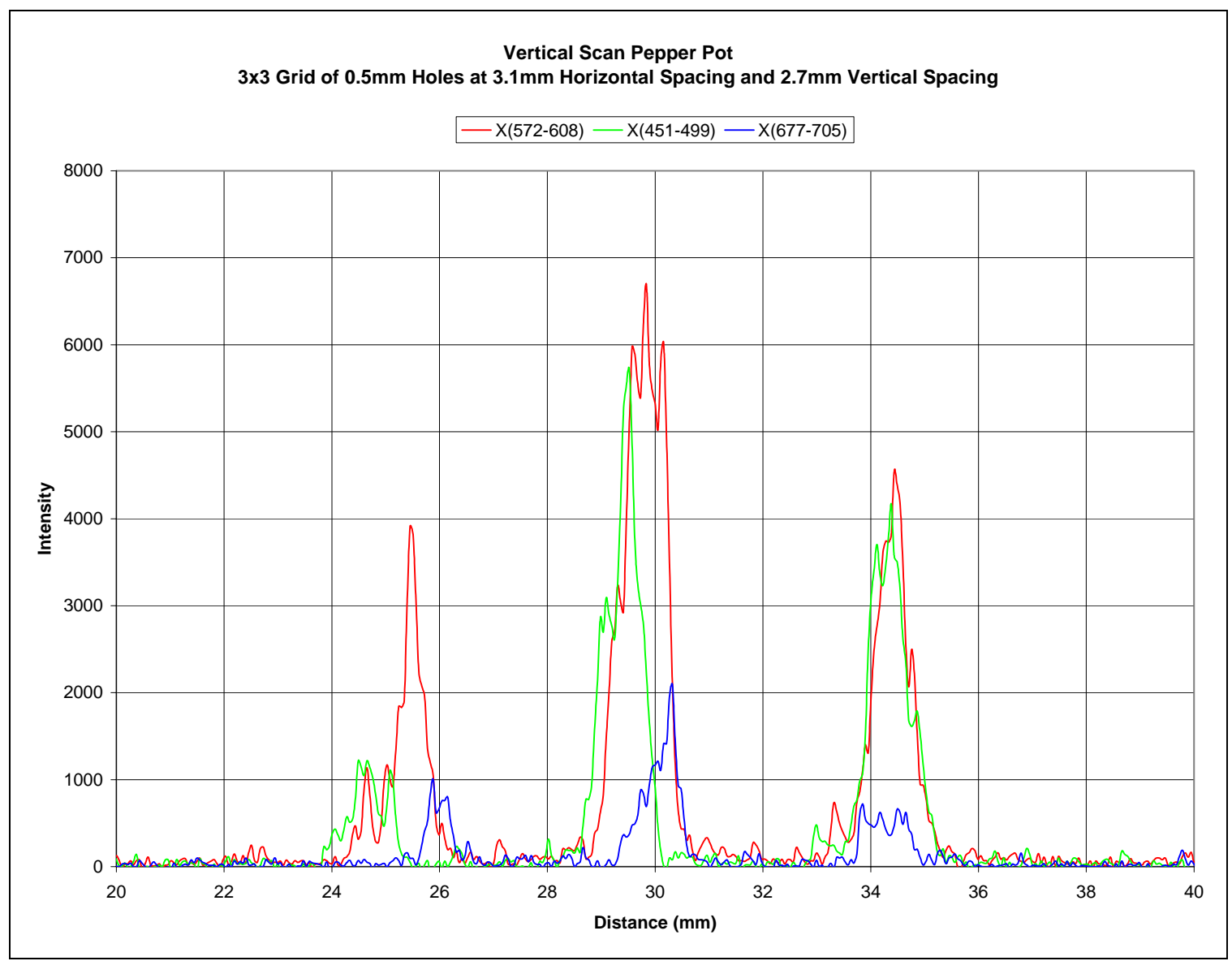

Figure 4.2.3.2: Line scans of pepper pot data. The lines are scans in the vertical direction, with horizontal positions chosen to go as nearly as possible through the centers of each of the three sets of spots. 


\subsection{Project Summary}

\subsection{Discussion of Source Characteristics Relative to HIF Requirements}

The results of the Phase II investigation of the pulsed inductive gas-breakdown ion source allow some clear conclusions as to the possible suitability of this source for ion accelerator applications. Fundamentally, this source is clearly proven capable of supplying Argon ion current density in the $100 \mathrm{~mA} / \mathrm{cm}^{2}$ range or higher. This meets the desired level for future HIF accelerator relevance. Although the source was not investigated with other ion species, the ionization and acceleration mechanism should be adaptable with no more than possible adjustment of voltages, to optimize performance with a wide variety of ion species. The low source temperature, as characterized by the lack of $\mathrm{Ar}^{2+}$ lines in the plasma spectrum and by the low measured transverse ion temperature $(0.6 \mathrm{eV})$ in the streaming plasma, imply that even species with lower secondionization energies than Argon should still be almost entirely in the singly-ionized state.

Reproducibility and spatial uniformity over a $5 \mathrm{~cm}$ diameter is a key figure of merit for HIF accelerator application. The Phase II final version of the source with axial puff valve and nozzle appears to be capable of operating at the $<5 \%$ level in reproducibility and $10 \%$ in uniformity, but it is not clear whether $<1 \%$ could be achieved.

Pulse length above $10 \mu$ s has been demonstrated. Control of the current density during the pulse has been demonstrated at the proof-of-principle level with the novel modulator coil method. This method, which allows attenuation of the ion current density without significantly affecting the beam quality, should be applicable to any streaming plasma ion source. Within the limits of source current density reproducibility, a modulator pulse shape can be chosen to flatten the current density during the desired duration of the pulse. This capability also implies that longer flat pulses, up to $20 \mu$ s or more, could be obtained by controlling the current density with the modulator to a lower level that could be supplied over a longer time by the source.

The acceleration experiments in Phase II were intended mainly as a means of producing a beam for diagnostic purposes. The accelerated beam enabled the time-of-flight (ToF) measurements for ion energy and charge state distribution, and the pepper-pot divergence data. The ToF measurements show the most serious deficiency in the accelerated beam, namely the presence of a significant amount of $\mathrm{Ar}^{2+}$ ions. It appears that the most likely source of these ions is not in the source. Rather, it appears that in the accelerating gap itself, due to poor ion optics that bombard the cathode, secondary electrons are produced, are accelerated across the gap and ionize some of the $\mathrm{Ar}^{1+}$ ions to produce the $\mathrm{Ar}^{2+}$. If this is the case, then the appearance of this contaminant species is not related to the ion source, but is a consequence of this high current-density accelerating gap design. Our design is certainly relatively unsophisticated, and there exist techniques to suppress the nonideal effects we have observed. In any case, this issue is inherent in any high currentdensity electrostatic acceleration configuration, independent of the ion source used. 
The attempt to measure transverse divergence of the beam with the pepper-pot diagnostic yielded the positive null result: within the sensitivity of the measurement, no growth in transverse divergence could be measured, beyond that expected to be produced by the accelerating electrode design.

\subsection{Accomplishments vs. Program Goals}

The program has successfully carried out the tasks initially envisioned to meet the program goals. Within the limits of diagnostics that could be implemented with the simple, relatively low-voltage, single acceleration stage that was attainable in this program, the performance of the source was well characterized.

All of the Phase I technical objectives were achieved. The final effort of Phase I, the analysis of the results from the source experiments with regard to accelerator requirements, indicated that the pulsed inductive plasma source could produce the desired ion source characteristics and justified further development in a Phase II program.

The technical objectives of the Phase II program were also achieved. The first objective, to determine the extent to which the ion pulse shape could be controlled, was achieved using an external modulator. Improvements in source uniformity and reproducibility, objectives 2, 3 and 4, were obtained by developing the axial valve and making modifications to the source geometry. Objective 5, an improved understanding of the source operation, resulted from an extensive study of the source with a nanosecond framing camera, spectrometer, magnetic field probes and Faraday cups. The final two objectives entailed the development of a beam extraction and acceleration stage and a study of the accelerated beam. The accelerator gap design and some diagnostic support were to be provided by a DOE national laboratory. Instead, as a result of changing priorities at the DOE laboratory, a first order accelerating gap design was produced inhouse. Additional diagnostics were either fabricated in-house or borrowed from the Laboratory of Plasma Studies at Cornell University to undertake the final Phase II objectives. The quality of the beam resulting from the first order accelerating gap design limited the resolution of some of the measurements carried out on the extracted beam.

\subsection{Future Directions}

This program has developed the inductively-driven gas-breakdown streaming ion source to a level of performance ready to proceed to detailed engineering for implementation on a particular ion accelerator. Indeed, the quality of the source is high enough that an accelerator is needed to quantitatively diagnose the source in terms of ion energy spread and beam emittance.

A fundamental remaining question about the source can only be answered by actually its actual use on an accelerator. This question concerns the coupling of the source of 
streaming ions, in the form of neutral plasma, to the initial extraction and acceleration gap of the accelerator.

This coupling is important to all aspects of the accelerator performance. The goal is to obtain optimal performance of the accelerating gap, that is, to extract the highest possible current density at the lowest possible divergence to make the brightest beam possible. To achieve this, the supplied ion current density in the plasma streaming into the anode must equal the extracted current density in the accelerated beam, thereby allowing the formation of a quasi-stationary plasma surface from which the ions are extracted at the anode. Only in this way can the electrostatic shape of the gap be maintained accurately. If the ion current density supplied to the anode is higher than the space-charge-limited current density that can be transported across the gap at the given accelerating voltage, then charge builds up at the anode and begins to fill the gap. This distorts the gap electric field, spreading the ion trajectories across the gap and degrading the beam quality. In our experiments this effect was the likely cause of gap breakdown: when ion optics is degraded so that the cathode is bombarded, electrons are produced, eventually resulting in breakdown. Conversely, if the ion current density is lower than the space-charge limit, the plasma will erode behind the anode, also changing the gap electrostatics.

Optimal design of the initial extraction and acceleration structure thus requires good control of the ion current density pulse shape. It is very encouraging that the modulator, invented in this program, provides a mechanism for control of this variable.

It would appear that substantial further development of this ion source depends upon implementation of the source in a real ion accelerator, capable of accelerating, transporting and diagnosing an ion beam at the high current $\left(100 \mathrm{~mA} / \mathrm{cm}^{2}\right)$ level the source provides. 


\section{References}

1. J.W. Kwan et al., "Ion sources and injectors for HIF induction linacs", Nuclear Instruments and Methods in Physics Research A 464 (2001) 379-387

2. "Pulsed Inductive Plasma Source Development for Advanced Propulsion Systems", S. C. Glidden, J. B. Greenly, M. Richter, Final Report, NASA Cont. NAS99052, June 1999.

3. J. B. Greenly, M. Ueda, G. D. Rondeau and D. A. Hammer, "Magnetically Insulated Ion Diode with a Gas-Breakdown Plasma Anode, "J. Appl. Phy 63, 1872 (1988).

4. "Thermal surface treatment using intense, pulsed ion beams," Mat. Res. Symp. Proc. 316 (1994) 521 (with R.W. Stinnett, R.G. Buchheit, F.A. Gruelich, C.R.Hills, A.C. Kilgon, D.C. McIntyre, M.O. Thompson, G.P. Johnston and D.J. Rej).

5. S.C. Glidden, J.B. Greenley and D.A. Hammer, "Compact Efficient 1kW Electric Thruster," NASA Contractor Report 191168, August, 1993. 


\section{List of Figures}

\section{Section 2 Summary of Phase I Program Results}

2.2.1. Block Diagram of the Plasma Source.

2.2.2. Section View of the Radial Gas Flow Source

2.2.3. Magnetic Field Plot for the Radial Gas Flow Source. 12

2.2.4. Relative Peak Ion Flux as a Function of the Time Delay Between the Valve and Ionization Coil Drivers.

2.2.5. Fast Ionization Gauge Measurement of the Gas Pressure.

2.2.6 Representative Ionization Coil Driver Waveforms, and PMT Signal from the Visible Light Output of the Plasma.

2.2.7. Collimated Aperture Faraday Cup

2.3.1 Angular Divergence Measurements.

2.3.2. Peak and Integrated Ion Flux For 10 and 20 Degree Nozzles at Different Delay Times.

2.3.3. Ion Flux (peak and integrated) $16 \mathrm{~cm}$ from the Source as a Function of Driver Voltage.

2.3.4 Superimposed Data from a 14-Shot Sequence.

2.3.5. Azimuthal Uniformity at Radii of 2 and $4 \mathrm{~cm}$.

2.3.6. Effects of Rotating the High Speed Valve 90 Degrees Counterclockwise. 23

2.3.7. Relative Peak Faraday Cup Signals for Delay Times of 95, 100 and $105 \mu \mathrm{s}$. 24

2.3.8. Radial Ion Flux Distribution as a Function of Distance from the Source. 25

2.3.9. Comparison of Ion Flux Measured $32 \mathrm{~cm}$ from Source at Radii of 0 and $2 \mathrm{~cm}$ for Different Ionization Coil Driver Voltages.

2.3.10. The Effects of Adding Damping Resistance to the Ionization Coil Driver Circuit on Source Output.

2.3.11 Effects of Truncating the Drive Pulse on the Output of the Ion Source.

\section{Section 3 Phase II Source}

3.1.1: Phase II Fast Gas Valve $\quad 35$

3.1.2: Side View of Source $\quad 36$

3.1.3: Driver Coil Configuration 37

3.1.4: Driver Pulsed Power 38

3.1.5: Timing of Front View Pictures Relative to Driving and Ion Currents 38

3.1.6: Front View Room Light $\quad 39$

3.1.7: Front View $2.90 \mu$ s $\quad 40$

3.1.8: Front View $3.10 \mu \mathrm{s} \quad 40$

3.1.9: Front View $3.52 \mu \mathrm{s} \quad 41$

3.1.10: Front View $3.82 \mu \mathrm{s} \quad 41$

3.1.11: Front View $4.32 \mu \mathrm{s} \quad 42$

3.1.12: Front View $4.52 \mu \mathrm{s} \quad 42$

3.1.13: Front View $4.72 \mu$ s $\quad 43$

3.1.14: Side View Room Light 43

3.1.15: Side View $3.90 \mu \mathrm{s} \quad 44$

3.1.16: Side View $4.14 \mu \mathrm{s} \quad 44$

3.1.17: Side View $4.30 \mu \mathrm{s} \quad 45$

3.1.18: Side View $4.74 \mu \mathrm{s} \quad 45$

3.1.19: Side View $4.90 \mu \mathrm{s} \quad 46$

3.1.20: Side View $5.06 \mu \mathrm{s} \quad 46$

3.1.21: Side View $5.22 \mu \mathrm{s} \quad 47$

3.1.22: Normalized B॰ Measurements with Plasma 48

3.1.23: Normalized B॰ Measurements without Plasma $\quad 48$

3.1.24: Normalized $B \circ$ Measurements at $Z=0.5 \mathrm{~cm} \quad 49$

3.1.25: $\mathrm{B}^{\circ}$ Measurements $\quad 50$

3.1.26: B॰ Measurements at a Shorter Delay $\quad 51$

3.1.27: Ion Flux for 4 Independent Pulses 


\section{List of Figures (continued)}

3.1.28: Variation in Ion Flux with Delay Timing 53

3.2.1: Modulator $\quad 54$

3.2.2: Modulation of the Streaming Plasma

3.2.3: Additional Modulation of the Streaming Plasma

3.3.1: Azimuthal Uniformity of the Phase II Source 56

3.3.1.1: Normalized Spectrum of Plasma Source at Different Timings between Gas Injection and Driver Current

3.3.1.2: Spectrum of Plasma Source at Different Timings between Gas Injection and Driver Current Focusing on Non-Ar Lines

3.3.1.3: Normalized Spectrum of Plasma Source at Different Timings between Gas Injection and Driver Current for wavelengths where lines of $\mathrm{Ar}^{2+}$ would occur.

3.3.1.4: Table Comparing NIST Relative Intensity and Spectral Data for $\mathrm{Ar}^{1+}$

3.3.1.5: Normalized Spectrum of Plasma Source at Different Source Driver Charge Voltages

3.3.1.6: Spectrum of Plasma Source at Different Source Driver Charge Voltages

3.3.1.7: Averaged and Normalized Spectrum of Plasma Source from Side and Forward Views

3.3.1.8: Averaged and Normalized Spectrum of Plasma Source from Side and Forward Views Focusing on $\mathrm{Ar}^{1+}$ lines

3.3.2.2: Normalized Current versus Faraday Cup Angle for Selected Radii at $32.3 \mathrm{~cm}$ from the Source

3.3.2.3: Absolute Current Density versus Faraday Cup Angle for Selected Radii at $22.3 \mathrm{~cm}$ from Source

3.3.2.4: Absolute Current Density versus Faraday Cup Angle for Selected Radii at $32.3 \mathrm{~cm}$ from Source

3.3.2.5: Single Standard Deviation Divergence Calculated Using Specified Gaussian Width

3.3.2.6: Observed Angle of Peak Ion Current versus Radius

3.3.2.7: Normalized Peak Ion Current versus Faraday Cup Angle for Selected Radii at $22.3 \mathrm{~cm}$ from Modulated Source

3.3.2.8: Peak Ion Current versus Faraday Cup Angle for Selected Radii at $22.3 \mathrm{~cm}$ from Modulated Source

3.3.2.9: Single Standard Deviation Divergence Calculated Using Specified Gaussian Width (modulated versus un-modulated)

3.3.2.10: Observed Angle of Peak Ion Current versus Radius (modulated versus un-modulated)

3.3.3.1: Ion Current versus Time at Different Distances from Source

3.3.3.2: Measured Time to Specified Ion Current Level

3.3.3.3: Calculated Velocity for Specified Ion Current Level

3.3.3.4: Calculated Time of Origin of Ion Current Using Time of Flight Data

\section{Section 4 Accelerated Beam Experiments}

4.1.1 Section view of apparatus for ion acceleration experiments.

4.1.2: Comparison of Acceleration Voltage using Step-Up Transformer and Solid State Marx Modulator

4.1.3: Ion Current Simulation for the First Design for the Acceleration Electrodes 75

4.1.4: Ion Current Simulation for the Second Design for the Acceleration Electrodes 76

4.1.5: Ion Current Simulation for the Third Design for the Acceleration Electrodes 77

4.1.6: Picture of Luminescence of Phosphor Screen Illuminated by Accelerated Ion Beam 78

4.1.7: Horizontal Line Fit of Luminescence of Phosphor Screen Illuminated by Accelerated Ion Beam

4.1.8: Spot Size of Luminescence of Phosphor Screen Illuminated by Accelerated Ion Beam 79

4.1.9: Simulated Divergence of Accelerated Ion Beam

4.2.1.1: Modulated Ion Current without Acceleration

4.2.1.2: Effects of Short Period Modulator Current Timing on the Ion Current without Acceleration 
Applied Pulsed Power, Inc.

\section{List of Figures (continued)}

4.2.1.5: Ion Current with Modulation and Acceleration with and without a Breakdown in the Acceleration Gap

4.2.1.6: Effects of Modulator Timing on Accelerated Ion Current 86

4.2.2.1: Faraday Cup Current at Two Different Z locations $\quad 87$

4.2.2.2: Calculated Arrival Times Given Known Acceleration Profile for $\mathrm{Ar}^{1+}$ and $\mathrm{Ar}^{2+}$

4.2.2.3: Faraday Cup Current at Two Different $Z$ locations for Different Source Plasma Driver Charge Voltages

4.2.3.1: Pepper pot data.

4.2.3.2: Line scans of pepper pot data. 
Applied Pulsed Power, Inc.

Appendix A

Phase I Final Report 


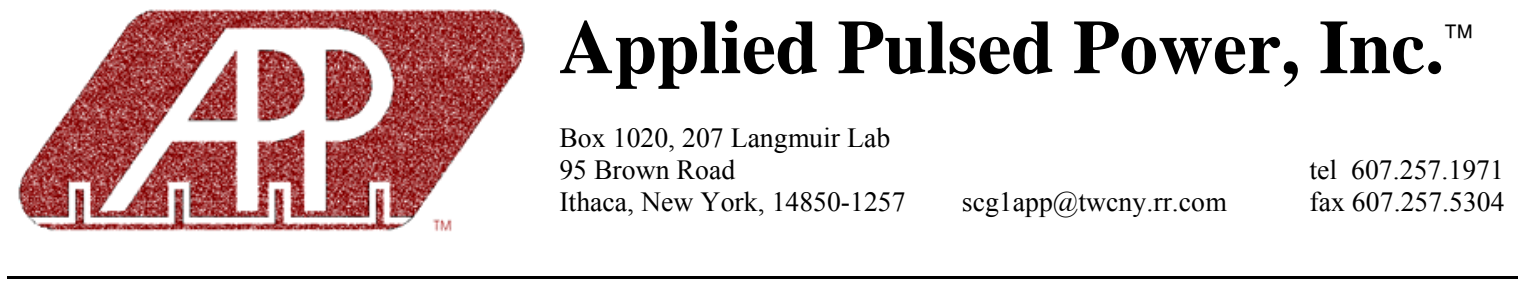

Pulsed, Inductively Generated, Streaming Plasma Ion Source for Heavy Ion Fusion Linacs

\author{
Steven C. Glidden \\ Applied Pulsed Power, Inc. \\ John B. Greenly, Daniel L. Dongwoo \\ Laboratory for Plasma Studies \\ Cornell University
}

March 14, 2002

U.S. Department of Energy Phase I SBIR Final Report

Grant DE-FG02-01ER83147 


\title{
Pulsed, Inductively Generated, Streaming Plasma Ion Source for Heavy Ion Fusion Linacs
}

\author{
Phase 1 Final Report
}

\section{Contents}

Introduction $\quad 3$

Streaming Plasma Ion Source Designs 4

Axial Gas Flow $\quad 5$

Radial Gas Flow $\quad 7$

Electrical Drivers $\quad 8$

$\begin{array}{lr}\text { Diagnostics } & 10\end{array}$

$\begin{array}{ll}\text { Relative Merits } & 10\end{array}$

$\begin{array}{ll}\text { Radial Gas Flow Ion Source Results } & 13\end{array}$

$\begin{array}{ll}\text { Directed Velocity } & 13\end{array}$

$\begin{array}{ll}\text { Divergence } & 15\end{array}$

Ion Current Density Dependence on Operating Parameters 18

$\begin{array}{ll}\text { Reproducibility } & 19\end{array}$

Azimuthal Uniformity $\quad 20$

$\begin{array}{ll}\text { Radial Distribution } & 23\end{array}$

Effects of Driving Waveforms on Source Performance 26

Plenum Pressure $\quad 30$

$\begin{array}{ll}\text { Charge State Measurements } & 31\end{array}$

Discussion of Results Relative to Phase I Goals and 31

the Requirements for Heavy Ion Fusion Accelerators

$\begin{array}{ll}\text { References } & 34\end{array}$

List of Figures $\quad 35$ 


\section{Introduction}

This Phase I SBIR project has developed a basic configuration for an inductively driven gas-breakdown plasma source that produces a $>10 \mu$ s pulse of Argon plasma of $>100$ $\mathrm{mA} / \mathrm{cm}^{2}$ ion current density, with strongly axially directed ion energy of about $80 \mathrm{eV}$, and sub-eV transverse temperature. The plasma is highly reproducible in its spatial, temporal and energy distributions. Several operating parameters, especially the driving voltage pulse shape, the distribution of gas provided by the pulsed valve to the source, and the geometry of the source and its driving coils, have been shown to strongly affect the plasma output and give the promise of being able to adjust the output to give high spatial and temporal uniformity. We believe that these two observed elements of the operation of this source, high reproducibility and the existence of "knobs" adjustable to optimize the output, make this source worthy of further development toward meeting the stringent requirements of a HIF ion source. The present project has used basic plasma diagnostics to characterize the streaming plasma output of the source, and we describe the results in this report.

Though with effort these measurements could certainly be brought to higher accuracy and precision than attained here, it is clear that all the usual limitations of plasma diagnostics make characterization of this source impractical at the level of accuracy needed to prove its performance with respect to HIF needs. The proper way to improve upon all the measurements of spatial and temporal distribution, energy spread and charge state distribution is to extract and accelerate a small $\left(\sim \mathrm{mm}^{2}\right.$ area $)$ beamlet from the plasma stream and use standard ion beam diagnostics on that extracted beamlet. Not only would this course avoid the limitations of measurement of the neutralized plasma, it would directly test the source in the true application, i.e. to supply the ions from which to extract a cold, bright ion beam. Emittance measurements could be made on such a beamlet. This would be the real test of the ability of any plasma ion source to supply a tightly controlled ion flux. 


\section{Streaming Plasma Ion Source Designs}

The Phase I experimental plasma ion source includes a high speed gas valve and its driver, a nozzle, ionization coils and their driver and related support hardware. Figure 1 is a block diagram of this source.

The high speed gas valve injects a puff of gas through a nozzle and into an annular volume between 2 driving coils. The coils are driven with a short period $(<3 \mu \mathrm{s}), 5-10$ $\mathrm{kV}$ ringing pulse. The current driven in the coils produces a rapidly rising magnetic field in the gas volume, inducing an azimuthal electric field in the gas of order $100 \mathrm{~V} / \mathrm{cm}$. This field initiates and drives a rapid breakdown in the gas and accelerates the resulting plasma.

The high speed gas valve is a standard APP product designed to generate annular gas puffs for the anode plasma source of an ion diode, and can operate at $>5$ pulses per second. The valve has a conical diaphragm that seals against an 'o' ring. A pulsed magnetic field is used to rapidly drive the diaphragm away from the 'o' ring, allowing gas from an annular plenum to flow radially outward. This results in a short $(<1 \mathrm{~ms})$ pulse of gas with a very fast $(<100 \mu \mathrm{s})$ rising leading edge. The valve used in Phase I has a plenum volume of 0.41 Torr liter at a plenum pressure of $3 \mathrm{~atm}, 0.23$ Torr liter at 1.6 atm and 0.084 Torr liter at $0.6 \mathrm{~atm}$. The timing between the high speed valve and ionization coil drivers is used to control the neutral gas density near the coils.

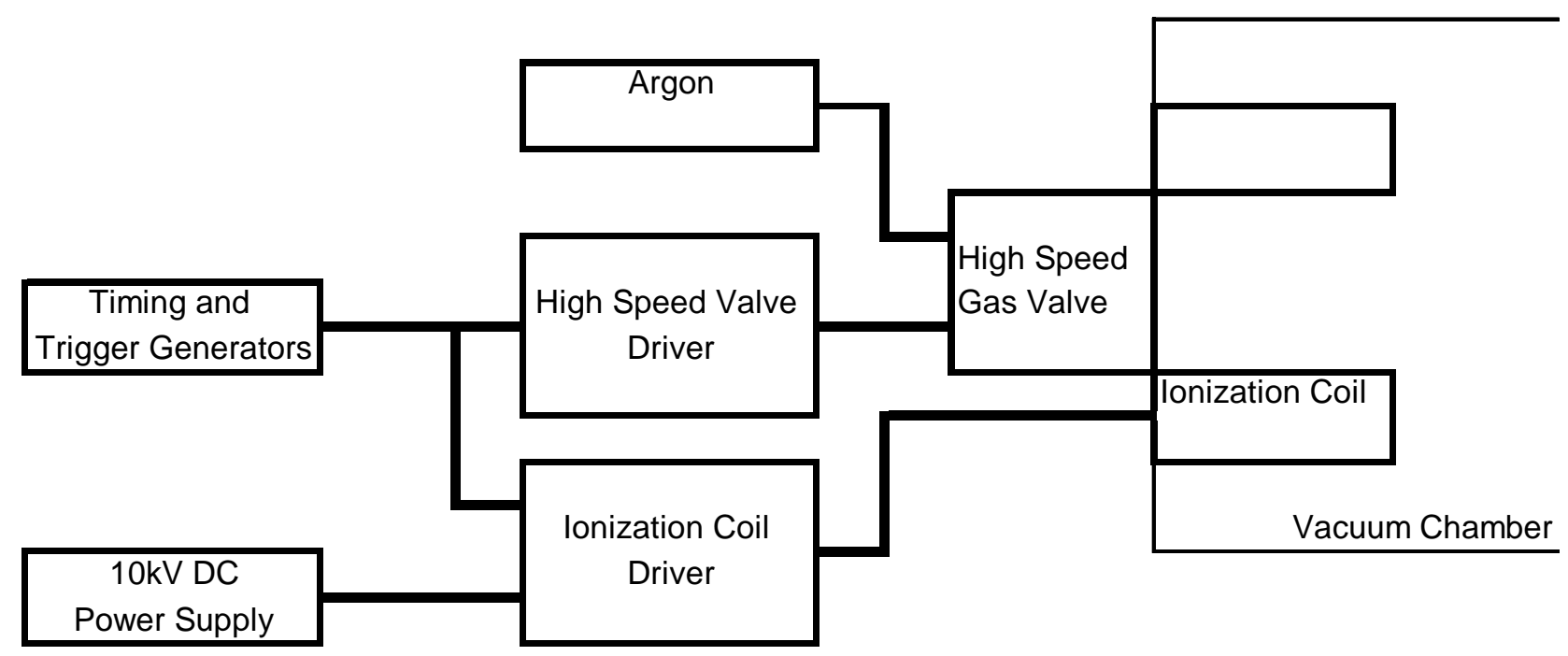

Figure1. Block diagram of the plasma source.

A characteristic of inductive ionization is the fact that without electrode phenomena that provide seed electrons (field enhancement and field emission, secondaries, photoelectrons) to begin the discharge, much higher electric fields may be required to 
initiate the ionization process. Since the ionization coil must have current driven in it to produce the changing magnetic flux that generates the inductive electric field, the higher that electric field must be (or the longer it must be applied) to initiate the ionizing discharge, the more energy must be expended in the electric driving circuit. Furthermore, unless a complex driving circuit is employed, an initial high voltage on the ionization coil leads to high peak current in the ringing LC waveform, which may far exceed the current needed in the discharge to drive the plasma to full ionization.

We have developed a simple method ${ }^{1}$ to provide an initiation mechanism that greatly reduces the driving voltage required. We add a single turn loop with a small gap located within the gas. This "preionizer" loop is inductively coupled to the ionizing coil, thus requiring no extra driving circuitry or energy input. By inserting a small $(<1 \mathrm{nF})$ capacitor in series with the loop, the loop current is limited to $<1 \mathrm{~A}$, so that plasma contamination and electrode erosion are essentially eliminated. The charge transferred through this gap is so small that its lifetime can be much greater than $10^{8}$ pulses. The very low-energy discharge across the gap triggers a distributed, volume discharge in the gas without significant current driven through the electrodes, while simultaneously eliminating the need for a higher electric field for pure inductive breakdown. The electrodes are used for what they do best - initiation. The inductive coil is used for what it does best; driving current without contamination or component wear.

The nozzle and ionization coil and preionizer winding forms are fabricated from plastic and the coils are epoxied in place. This fabrication method is reliable, quick and inexpensive, and allowed substantial modifications and the fabrication of new coils during the course of the experiments.

Several different nozzle and coil geometries were explored in Phase I. We will briefly describe and contrast these different sources here. A more detailed discussion of the performance of the most successful approach will follow.

\section{Axial Gas Flow Ion Source}

The first source used a nozzle geometry that directed the gas flow into an annular volume between 2 driving coils (Figure 2). Each coil has two closely spaced turns and all turns are connected in series. The magnetic field null from the driving coils is located in the gas volume. With this magnetic field configuration, ions will be accelerated both upstream and downstream of the coils. Typical ionization coil driver current and voltage waveforms are shown in Figure 3. Also shown are the signal from a photomultiplier tube (PMT) that monitors the visible light output of the plasma, and the ion current density measured by a Faraday cup located $12 \mathrm{~cm}$ downstream from the source. 


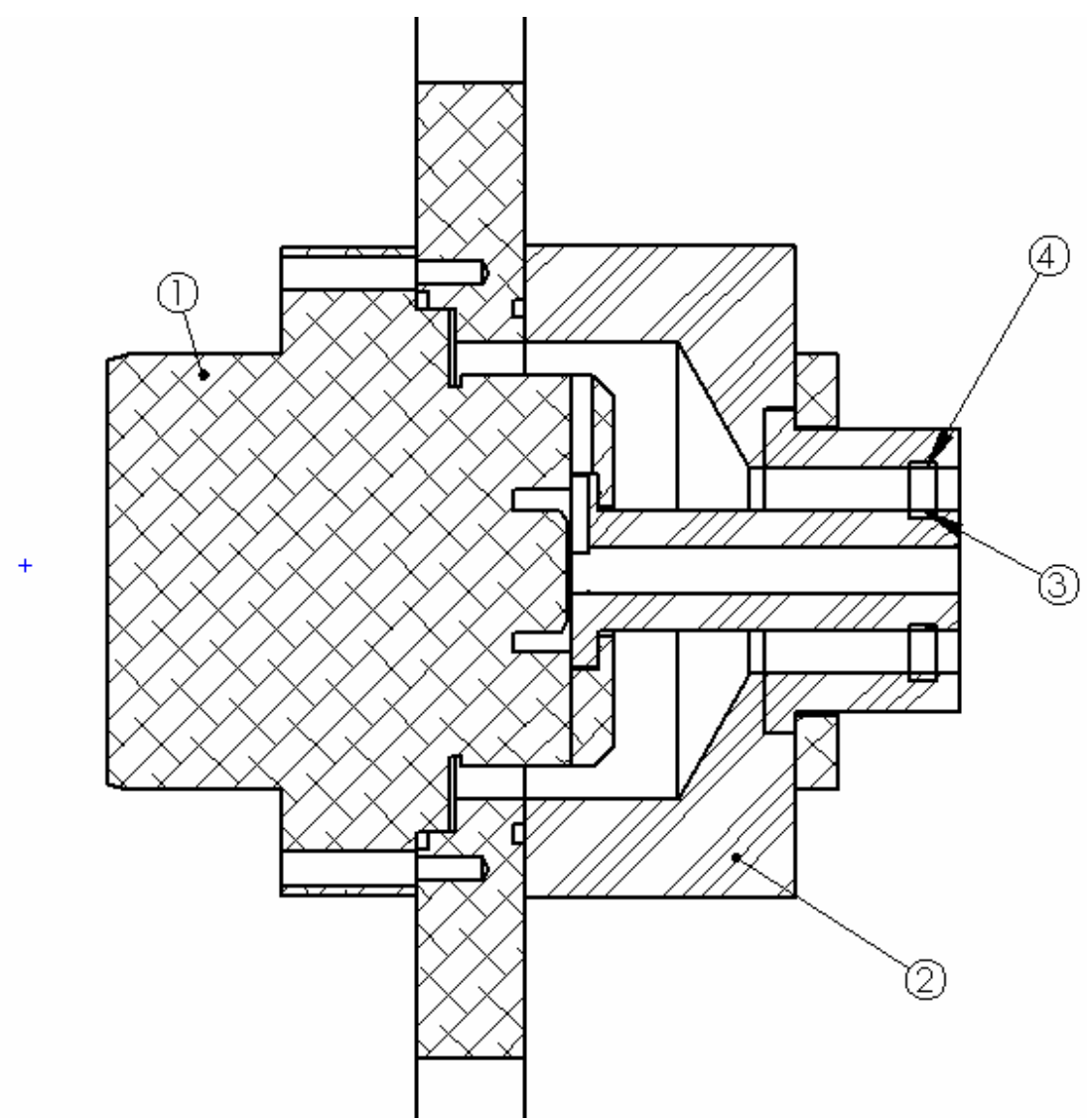

(a) Section view of axial gas flow ion source.

1-High speed valve, 2-nozzle, 3-inner coil, 4-outer coil.

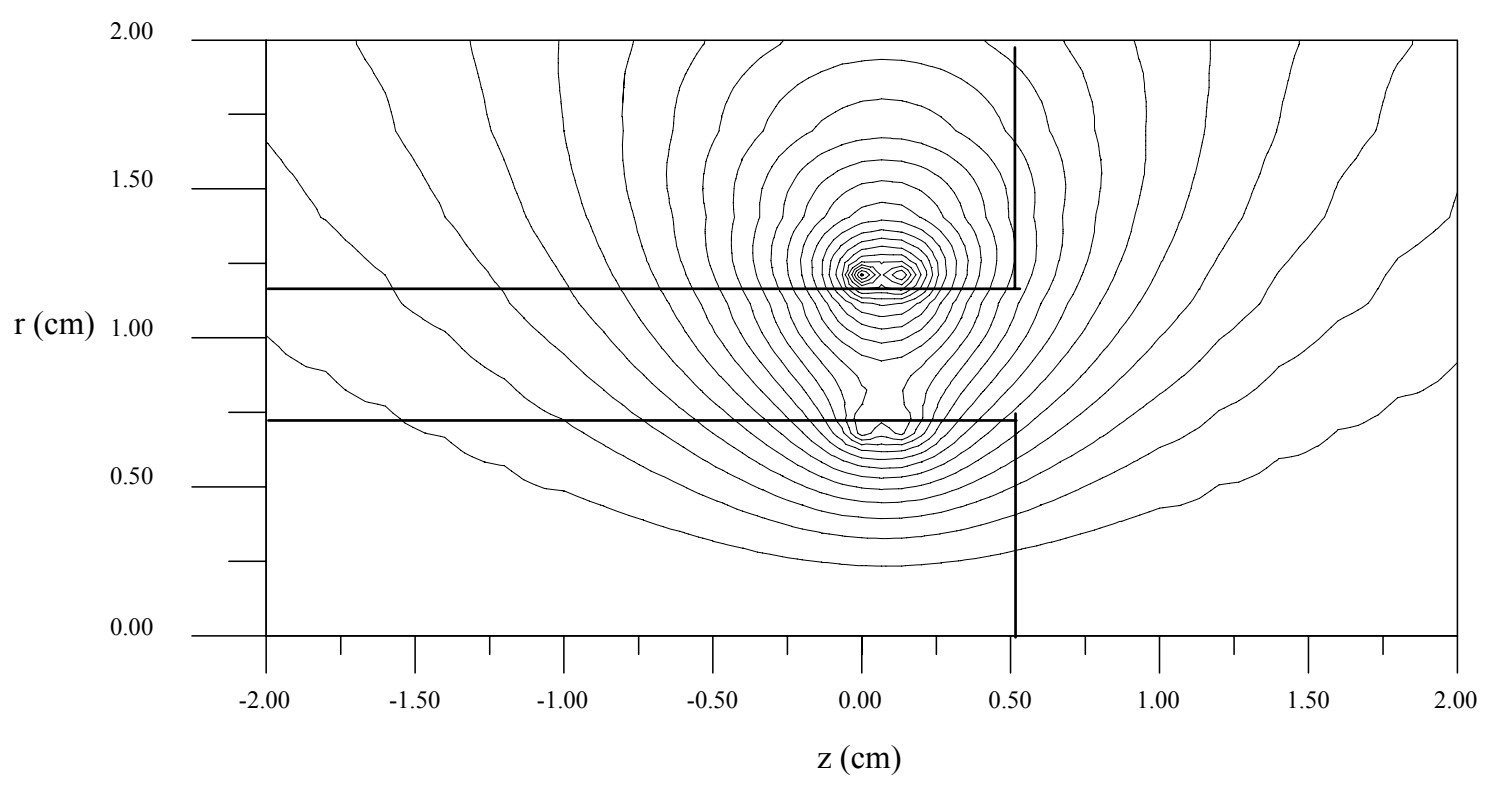

(b) Magnetic field plot

Figure 2 Axial gas flow ion source 


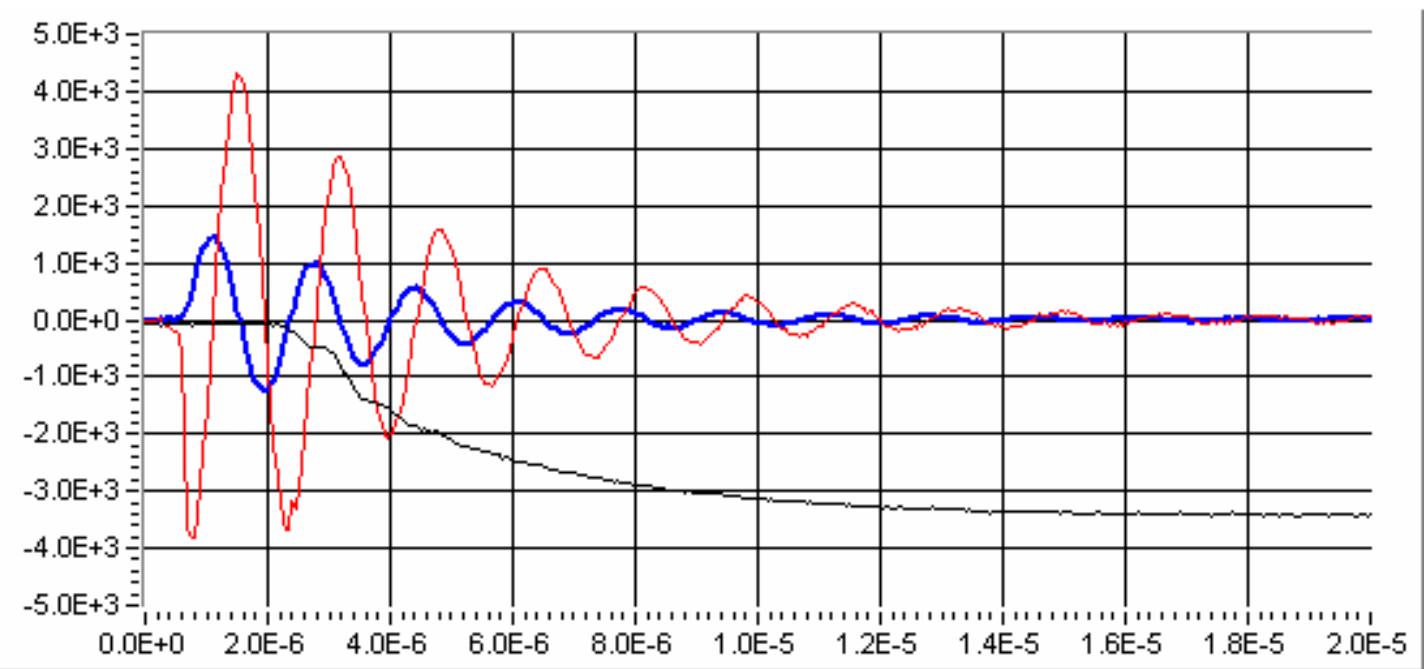

(a) Ionization coil driver output voltage, V, current (bold) A, and Photomultiplier signal

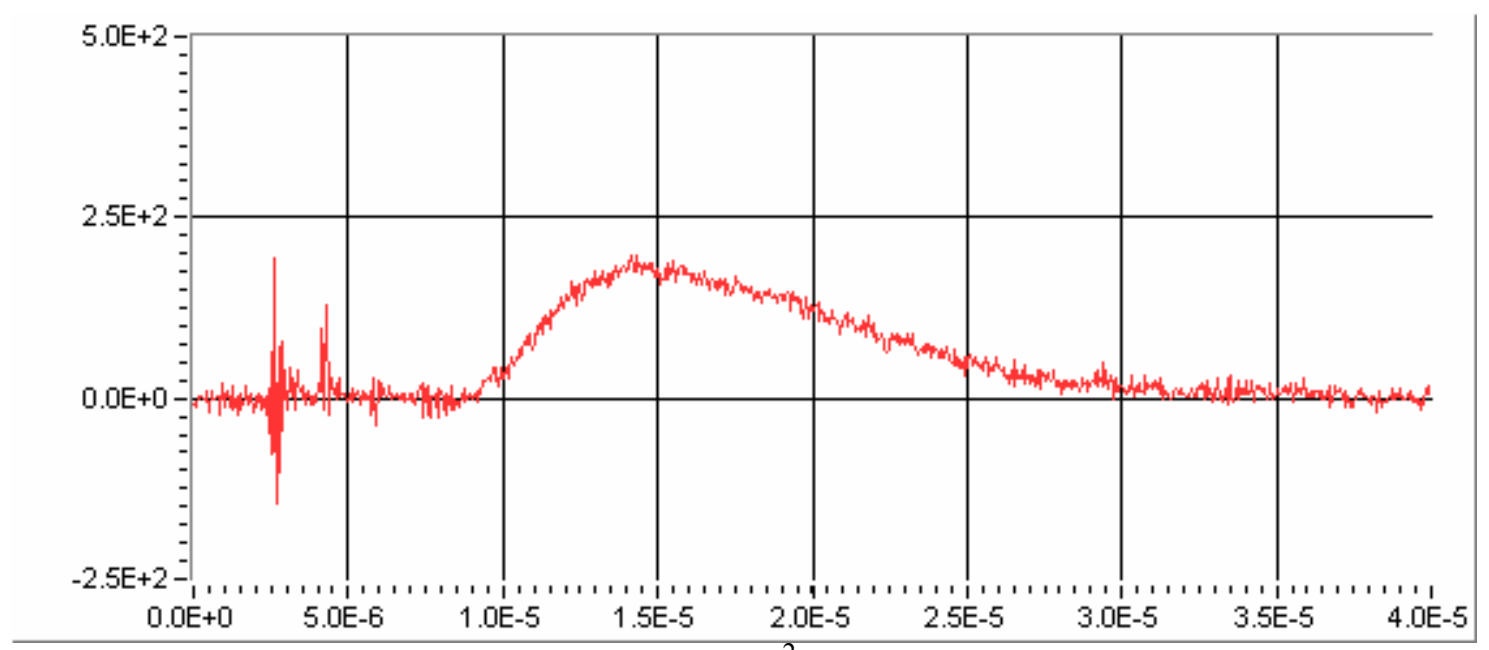

(b) Faraday cup signal $\left(\mathrm{mA} / \mathrm{cm}^{2}\right)$; cup $12 \mathrm{~cm}$ from source.

Figure 3 Ionization coil driver waveforms, photomultiplier tube signal from the visible light output of the plasma, and the ion current density waveform for the axial gas flow source. 76

Variations of this geometry included increasing the axial spacing between the turns of each coil, operating with 3 turn outer and 1 turn inner coils, and replacing the outer turns with a $2 \mathrm{~cm}$ long conical sheet winding.

\section{Radial Gas Flow Ion Source}

The radial gas flow source uses the nozzle to generate an annular ring of gas that has a very steep gradient in the axial direction. The purpose of this configuration is to 
minimize the amount of neutral gas downstream from the ionization region. Two versions this configuration were explored.

For one version the ionization coils were located on the downstream side of the nozzle to keep the location of the magnetic field null within the gas volume. As with the axial source, ions will be accelerated in both axial directions.

The version which produced the greatest ion current downstream of the source has coils located upstream of the nozzle and is shown in Figure 4. All ions are accelerated downstream. The magnetic field null is now in the plastic coil form near the surface so breakdown may be initiated at this surface. However, it is possible to remove material in this region if it is necessary to eliminate surface contamination. This was not done in Phase I.

The version of the source shown in Figure 4 is similar to the ion source used to generate high current $(>10 \mathrm{kA})$ hydrogen and nitrogen ion beams for materials modification ${ }^{2}$.

Each coil has a single turn and both turns are connected in series. Because the aluminum valve body acts as a flux excluder, the coils are larger in radius than those for the axial flow source $(\sim 4 \mathrm{~cm}$ vs. $\sim 1 \mathrm{~cm})$. If it is desirable to shrink the size of the source, smaller valves could be and have been built in the past. We will discuss other options for future development in this proposal.

\section{Electrical Drivers}

The ionization coil driver uses a solid state switch to discharge a capacitor charged to $<10 \mathrm{kV}$ through the ionization coils. The output capacitance and thus the ringing frequency of the ionization coil current is varied by adding additional capacitors. The driver has resistive voltage monitors, and Rogowski coils for measuring output current. An additional switch was added to enable the ringing discharge to be truncated after a few cycles.

The high speed valve driver is a standard APP product and generates a few kA, $30 \mu$ s pulse which opens the valve.

The timing between the high speed valve and ionization coil drivers is independently adjusted. 
Applied Pulsed Power, Inc.

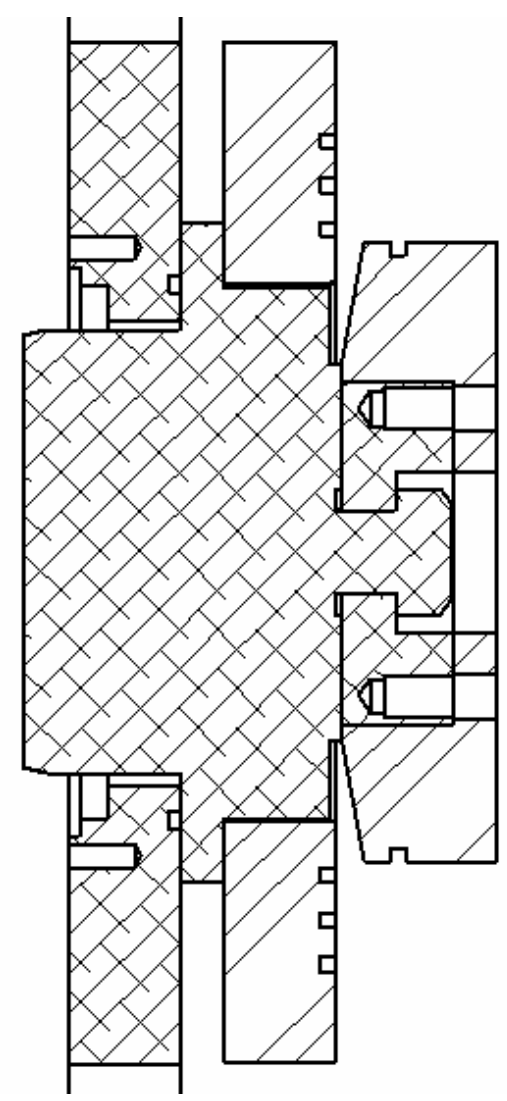

(a) Section view

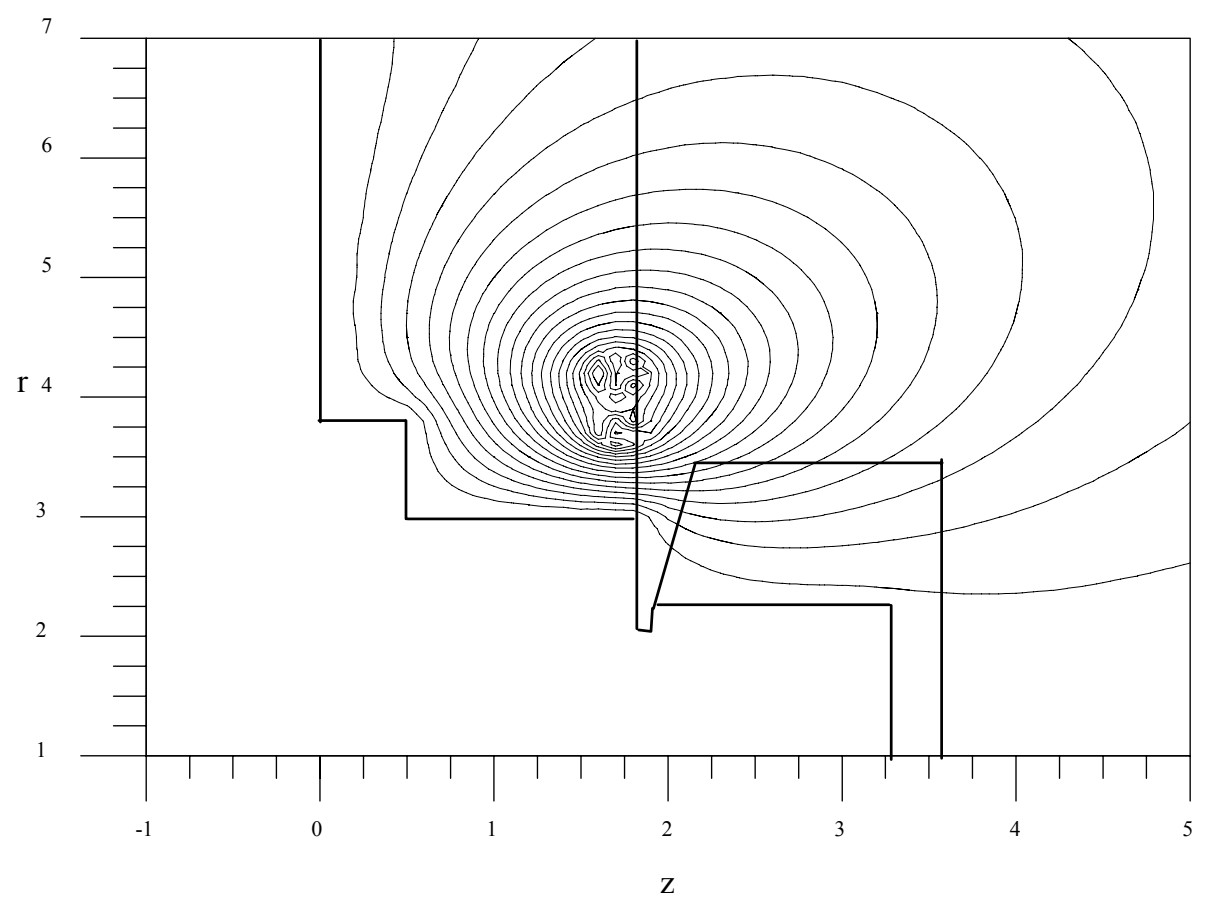

(b) Magnetic field geometry

Figure 4. Radial gas flow source. 


\section{Diagnostics}

The primary diagnostic used in exploring the behavior of the source were collimated aperture Faraday cups. The Faraday cups had $1 \mathrm{~mm}$ diameter apertures, with a second positioned several $\mathrm{cm}$ in front. This configuration is shown in Figure 5. Unless otherwise noted, the ion flux measurements presented in this report used this configuration.

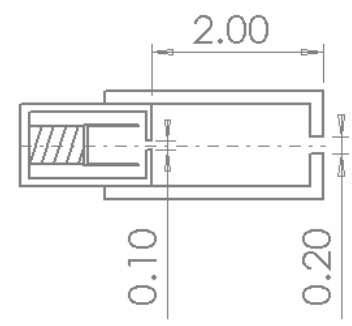

Figure 5. Collimated aperture Faraday cup; dimensions in $\mathrm{cm}$.

A photomultiplier tube measured the onset of visible light from the breakdown of the argon gas.

\section{Relative Merits}

Measurements of the axial and radial gas flow sources demonstrated similar performance in terms of directed velocity, beam divergence and reproducibility. However, the radial sources were capable of generating several times greater ion flux as a result of the considerably steeper axial density gradient of the gas from the radial flow nozzle.

The ion flux from the source rapidly decreases as a result of collisions between the ions and the gas molecules, when the density of the neutral gas in front of the source exceeds 10 mTorr. This behavior can be seen in Figure 6, which shows the peak ion flux measured $16 \mathrm{~cm}$ from the source at different valve to ionization coil driver delay times for the axial flow source. For delays longer than $210 \mu \mathrm{s}$, the ion flux quickly falls to zero, even though plasma is generated for delays $>300 \mu$ s as observed by the photomultiplier. 


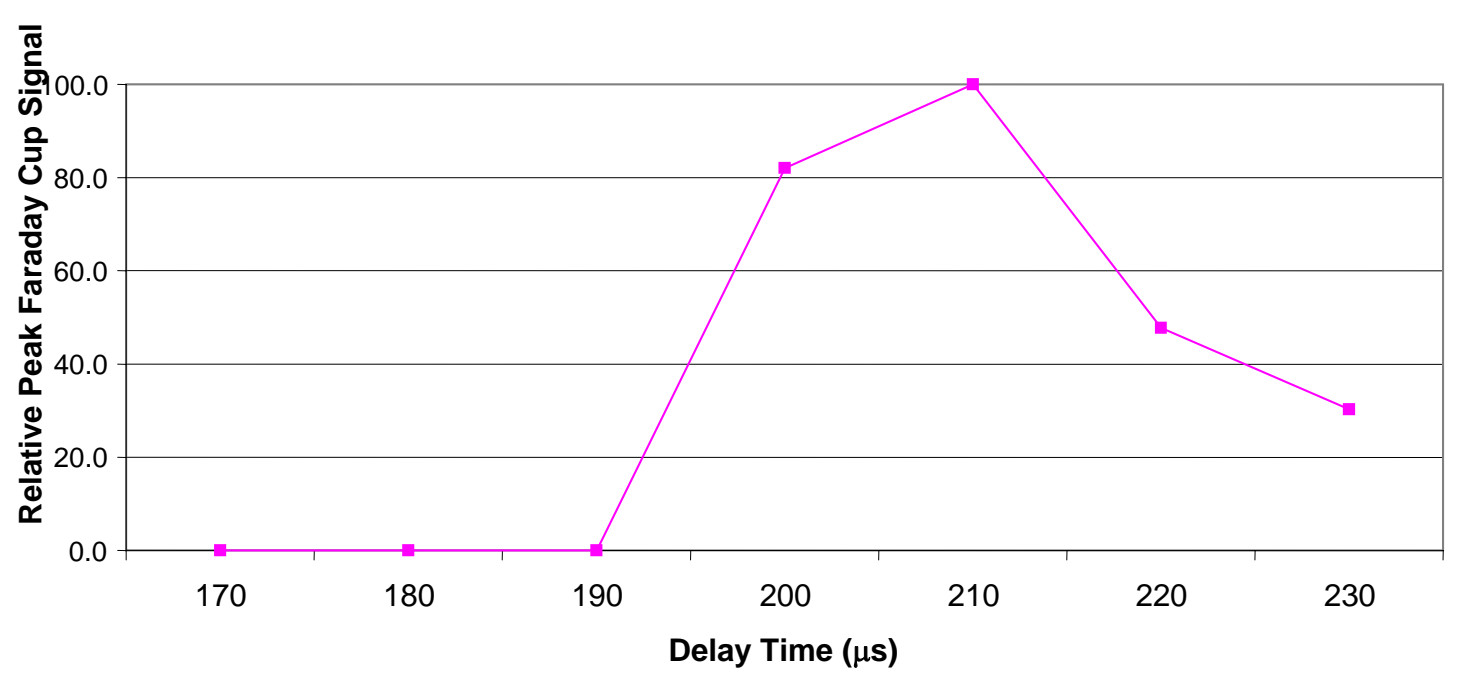

Figure 6. Relative peak ion flux as a function of the time delay between the valve and ionization coil drivers.

The gas pressure near the ionization coils for each source was measured using a Fast Ionization Gauge (FIG) (Figure 7). The FIG samples a region of gas of $3 \times 3 \times 10 \mathrm{~mm}$ without obstructing the flow of gas in the desired direction. The FIG response was nonlinear with argon above $\sim 50$ mTorr, so the peak pressures indicated in the figure are approximate.

With the axial gas flow source, the axial gradient of the gas density is not very steep; the gas is forced to flow around two corners, and gas flow in this pressure regime is quite viscous, resulting in subsonic, rapidly spreading flow in this nozzle. In order to obtain substantial ion flux downstream of this source, it must be operated using the leading edge of the gas pulse to minimize gas density downstream. 


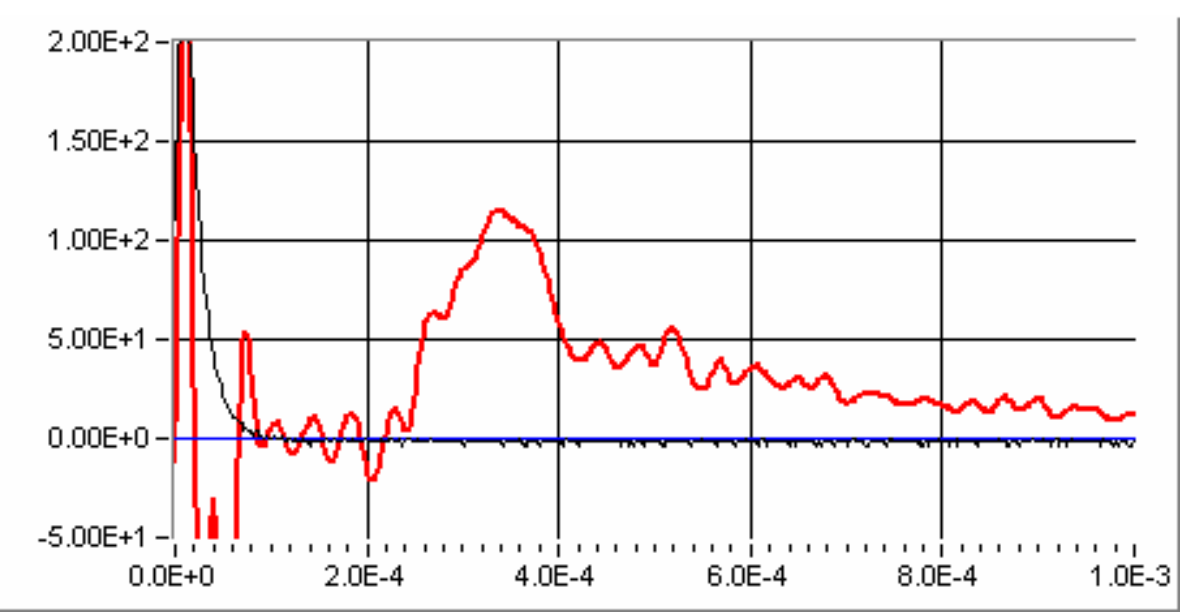

(a) Gas pressure (mTorr) measured $3 \mathrm{~cm}$ from end of axial gas flow source. 139

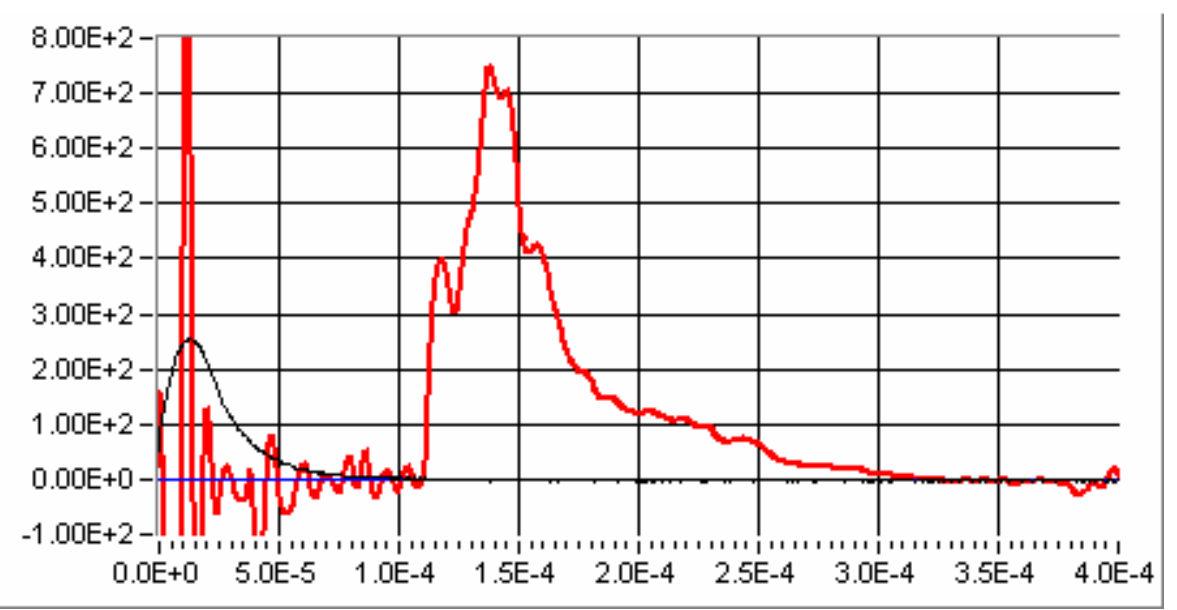

(b) Gas pressure (mTorr) measured at a radius of $4 \mathrm{~cm}, 0.5 \mathrm{~cm}$ from the radial gas flow source. Note different pressure and time scales from (a). 157

Figure 7. Fast ionization gauge measurements of the gas pressure. The first $100 \mu$ s of the waveforms are noise pickup from the valve driver current (light trace).

Attempts at ionizing a longer slug of gas by increasing the spacing between turns for each coil, resulted in only slightly more flux. Figure 8 compares the performance of these two versions of the axial gas flow source for different driving voltages. The increase in ion flux with higher driver voltage begins to level off above $8 \mathrm{kV}$. 


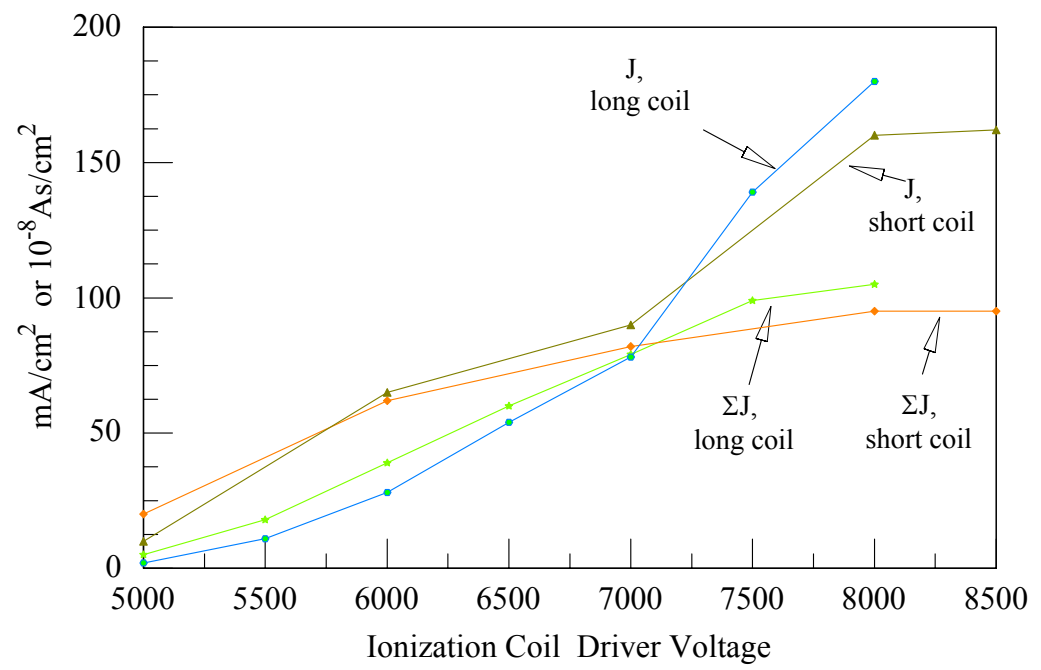

Figure 8. Comparison of axial gas flow source performance for coils with closely spaced (short coil) and widely spaced (long coil) turns. Faraday cup located on axis, $12 \mathrm{~cm}$ from source.

The radial flow sources, on the other hand, have a very steep axial gradient as a result of the design of the gas nozzle, which gives supersonic flow in this regime. The gas density therefore can be much higher in the region of the coils without attenuating the axial flow of the ions downstream. Substantially greater fluxes are obtained with the radial sources. For example, the ion flux measured on axis $12 \mathrm{~cm}$ from the source with the driver charged to $8 \mathrm{kV}$, ranged from $160-180 \mathrm{~mA} / \mathrm{cm}^{2}$ for the axial gas flow sources compared to $490 \mathrm{~mA} / \mathrm{cm}^{2}$ for the radial flow version.

Another advantage of the radial flow source is that in an accelerator, the ion extraction and acceleration electrodes could be much closer to the source without contamination from neutral gas, because most of the supersonic gas flow exits radially near the source and could be optimally pumped there.

Because of its observed advantages, the radial gas flow configuration was chosen for detailed experimental measurements. Its performance will be discussed more extensively in the remainder of this section.

\section{Radial Gas Flow Ion Source}

\section{Directed Velocity}

Signals from Faraday cups separated by $8 \mathrm{~cm}$ are shown in Figure 9. The ion velocity inferred from this data is $\sim 2 \mathrm{~cm} / \mu \mathrm{s}$. By comparison, the thermal velocity of the argon gas is $\sim .04 \mathrm{~cm} / \mu \mathrm{s}$ at $20 \mathrm{C}$. 
Applied Pulsed Power, Inc.

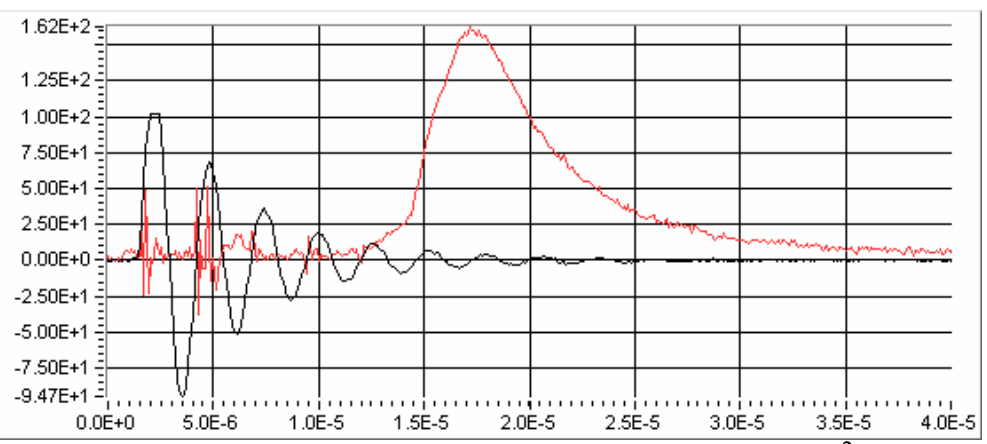

Ionization coil current $(\mathrm{x} 0.1 \mathrm{~A})$ and Faraday cup signal $\left(\mathrm{mA} / \mathrm{cm}^{2}\right)$ at $4 \mathrm{~cm}$ radius.

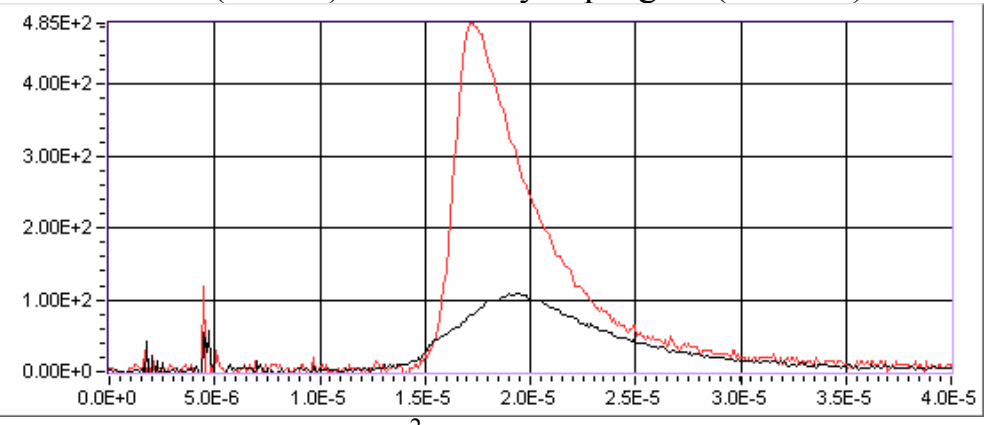

Faraday cup signals $\left(\mathrm{mA} / \mathrm{cm}^{2}\right)$ at radii of 0 (large signal) and $2 \mathrm{~cm}$.

(a) Faraday cups at $\mathrm{z}=16 \mathrm{~cm} .351$

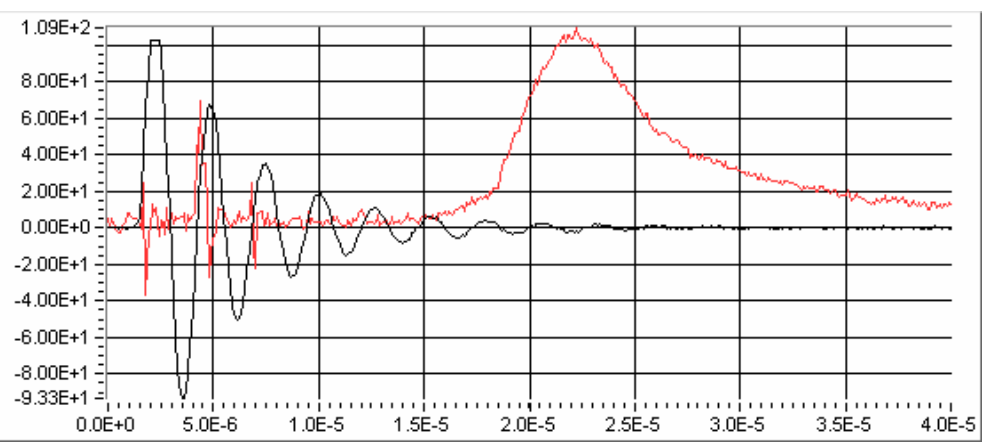

Ionization coil current $(\mathrm{x} 0.1 \mathrm{~A})$ and Faraday cup signal $\left(\mathrm{mA} / \mathrm{cm}^{2}\right)$ at $4 \mathrm{~cm}$ radius.

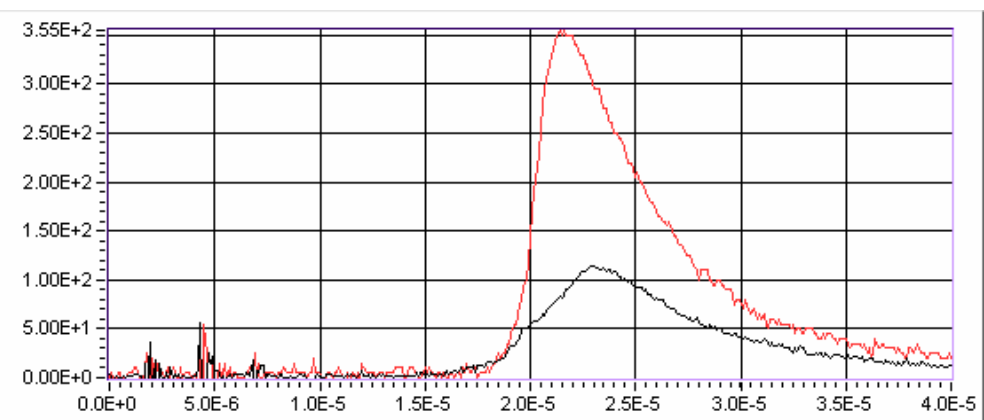

Faraday cup signals $\left(\mathrm{mA} / \mathrm{cm}^{2}\right)$ at radii of 0 (large signal) and $2 \mathrm{~cm}$.

(b) Faraday cups at $\mathrm{z}=24 \mathrm{~cm} .345$

Figure 9. Signals from Faraday cups separated by $8 \mathrm{~cm}$ 


\section{Divergence}

An array of Faraday cups with collimated apertures as shown in Figure 5 was used to examine beam divergence. The axis of collimation could be tilted at various angles relative to the axis of the source. The good reproducibility of the source enabled us to compare Faraday cup signals taken at 2 degree intervals from -10 to +10 degrees.

The resulting signals were analyzed in the following way to find the angular divergence of the ions in the streaming plasma. A Gaussian distribution was assumed for the intensity of the ion current density versus angle, corresponding to a thermal (maxwellian) transverse ion velocity distribution. Taking into account the finite acceptance angle of the collimation, a Mathcad worksheet was written that generates a curve for each angle of tilt. The curve plots the signal that would be obtained at that angle (normalized to the signal at zero angle) as a function of the Gaussian width shown as standard deviation (Figure 10 a). The experimental data point for a given angle is then located on the curve to find the corresponding angular Gaussian standard deviation. The results are shown in Figure 10b. There is a pair of points at each angle; these result from a pair of measurements taken at that angle on opposite sides of zero degrees.

The results of Figure 10b show that all angles from 4-10 degrees were consistent with a width of $5.7+/-0.5$ degrees (Figure $10 \mathrm{~b}$ ). At 2 degrees, the finite collimator acceptance angle results in a very insensitive measurement, producing too large an uncertainty for a useful fit. Excluding 2 degrees, the data fit the 5.7 degree Gaussian standard deviation well within the measurement precision of $\sim 1$ degree.

Taking the axial velocity of $2 \mathrm{~cm} / \mu$ s gives $\sim 80 \mathrm{eV}$ axial energy for Ar+ ions, and this standard deviation of 0.1 radian therefore corresponds to a transverse velocity one tenth of the axial velocity, or $\sim 0.8 \mathrm{eV}$ transverse ion energy. Similar sets of measurements under other operating conditions and distances from the source gave results from $0.5 \mathrm{eV}$ to $0.8 \mathrm{eV}$. Use of the usual Maxwellian thermal velocity definition thus gives $0.4-0.6 \mathrm{eV}$ ion "temperature".

It is necessary to comment further on the meaning of this measurement. The axially streaming plasma passes through the first aperture of the collimator and then expands as it travels to the second aperture. This expansion is caused by two factors. The first is the transverse energy spread of the ions in the stream that enters the aperture, and if this were the only effect, the $0.6 \mathrm{eV}$ number would be a correct characterization. The second effect is that even in the absence of any transverse spread in the entering ion distribution, the plasma stream that is selected by the first aperture would be expected to expand transversely due to electron thermal pressure at the ion sound speed, which goes as $\left(\mathrm{Te} / \mathrm{m}_{\mathrm{i}}\right)^{1 / 2}$. Thus, from this point of view, the expansion is a measure of Te. If the ions are much hotter than the electrons, the first effect dominates, but if the Te $\sim \mathrm{Ti}$, the electron effect dominates. If $\mathrm{Te}=\mathrm{Ti}$, this speed is faster than the ion thermal speed by the square root of the adiabatic index gamma, which for a single-species ion-electron plasma in this free transverse expansion, should be a factor of $\sim 1.3$. Thus the inferred $0.6 \mathrm{eV}$ is an upper limit for the ion transverse ion temperature. The ions could be colder than this, 
and the expansion in the collimator dominated by the electron temperature, though this is probably unlikely in this plasma. In any case, the low temperature is the consequence of the relatively "gentle" inductive driving of the gas ionization, which is essentially a pulsed, relatively low-frequency RF discharge, and possibly also some cooling as the plasma flows away from the source. More detailed measurements in Phase II will be made in the source region to fully characterize the gas breakdown regime in this source, and identify the conditions which minimize the ion temperature. 


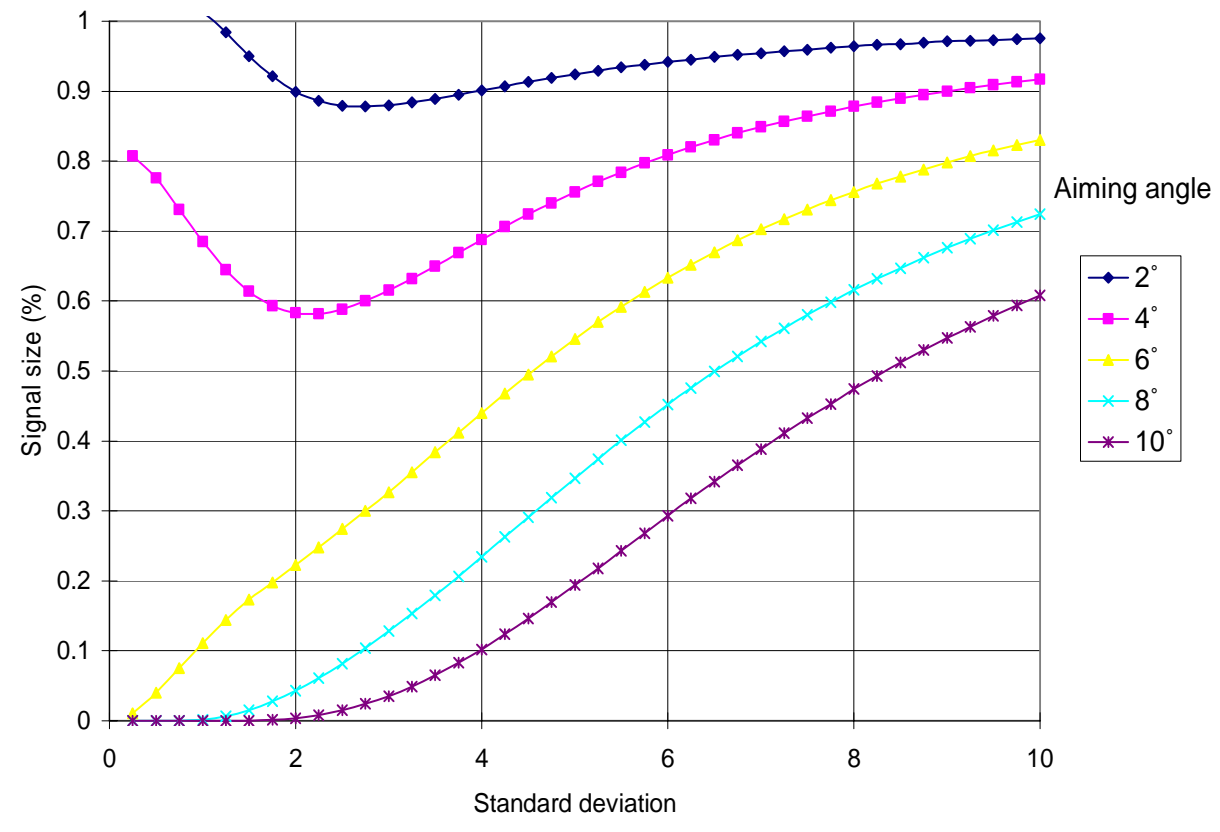

(a) Mathcad generated plots of the signal that would be obtained at different angles of tilt (normalized to the signal at zero angle) as a function of the Gaussian width.

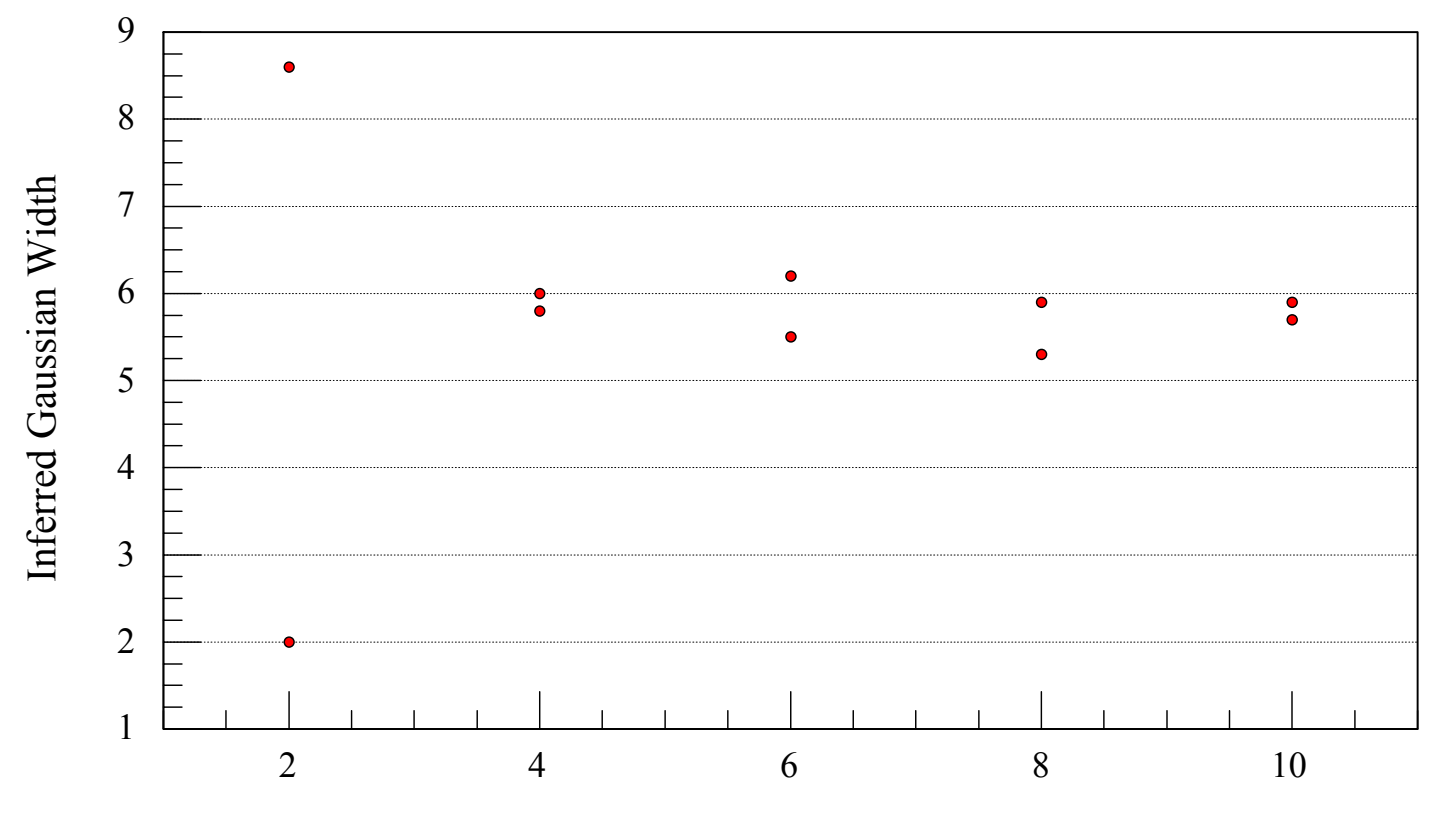

Angle of Measurement

(b) Inferred Gaussian standard deviation at different angles of tilt comparing collimated Faraday cup measurements with the plots in (a).

Figure 10 Angular divergence measurements. 


\section{Ion Current Density Dependence on Operating Parameters}

The design of the gas flow nozzle affects the output of the source. This is seen in Figure 11, which compares for the radial flow source, the output at $16 \mathrm{~cm}$ for 2 simple nozzle designs at different valve to coil delay times. The 10 degree nozzle produces a thinner layer of gas in front of the coils, achieving maximum ion flux at a longer delay time when there is a higher density in front of the ionization coils.
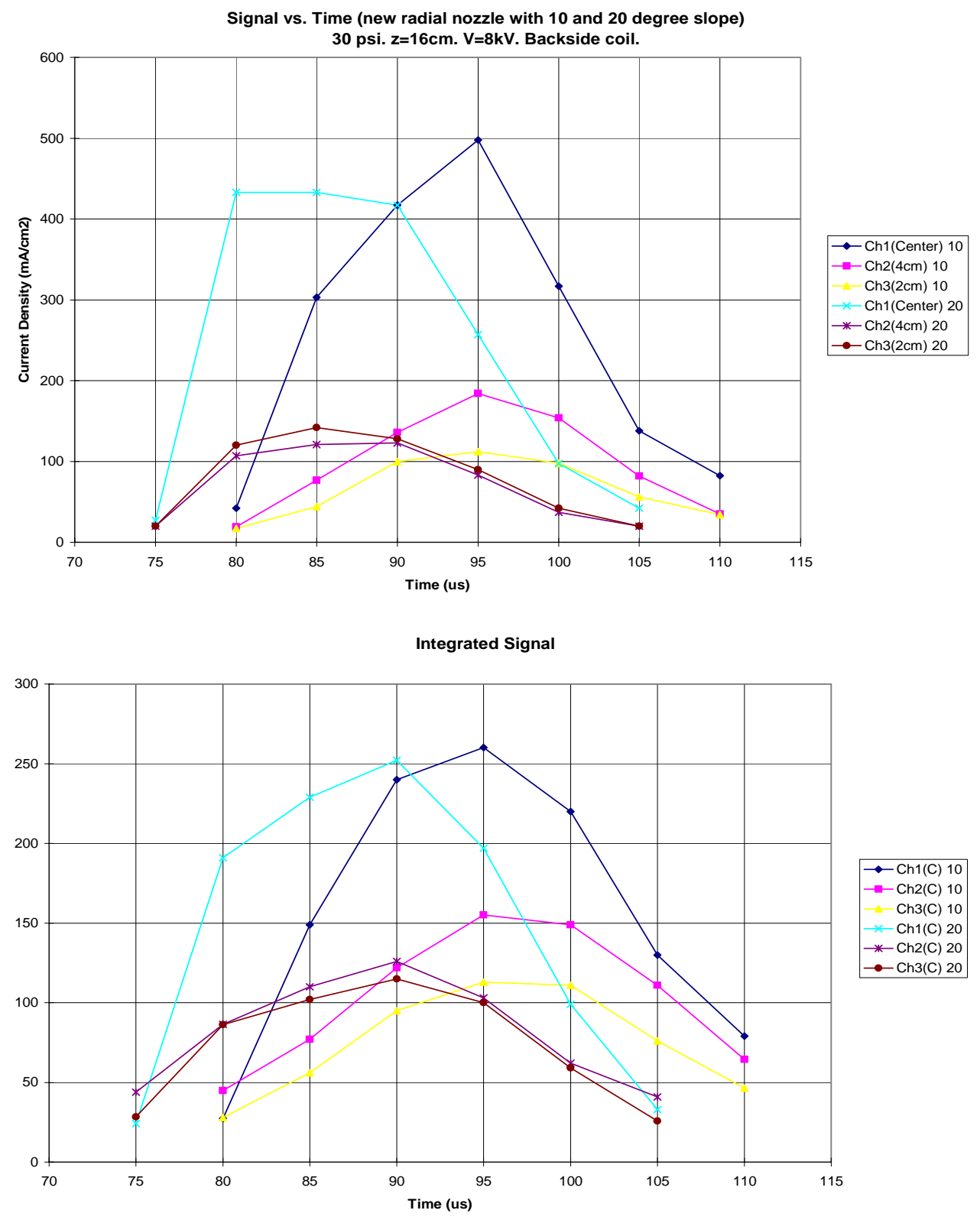

Figure11. Peak and integrated ion flux for 10 and 20 degree nozzles at different delay times.

Both the peak ion flux and the integral increases as the ionization coil driver voltage is increased. This is seen in Figure 12, with the Faraday cup located $16 \mathrm{~cm}$ from the source. 
Up to $9 \mathrm{kV}$, the maximum voltage possible with our present driver, the ion flux is still increasing with increased voltage.

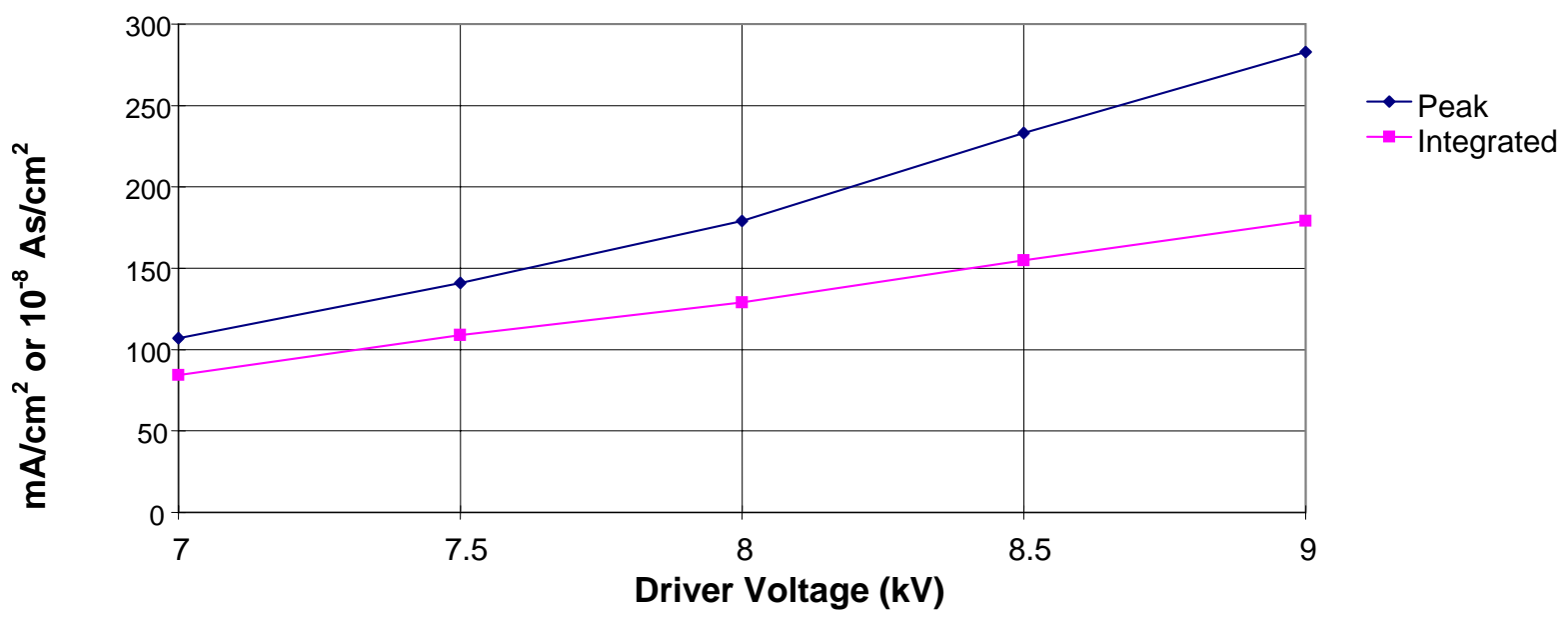

Figure 12. Ion flux (peak and integrated) $16 \mathrm{~cm}$ from the source as a function of driver voltage.

\section{Reproducibility}

The reproducibility of the source was observed by operating at a 2 pulse per minute repetition rate. The digital oscilloscopes were set to operate in the envelope mode, wherein the maximum and minimum values of the signal at each time interval are stored. Irreproducibility could occur as a result of surface contamination of the plastic surfaces on the coil forms from the untrapped oil diffusion pump and other sources. Other sources of irreproducibility include variations in the performance of the high speed valve, and/or the valve and coil drivers and changes in the valve plenum pressure. Results are shown in Figure 13 for a 14-pulse sequence. These data are for operation at $8 \mathrm{kV}$ with a $100 \mu \mathrm{s}$ delay time. Similar results were obtained with the driver operated at different voltages. The upper and lower limits of the envelope of multiple signals are actually dominated by the amplitude of the noise on any one signal, so a more sophisticated measurement technique will be necessary in order to quantify reproducibility below the approximately $5 \%$ level in these envelope data. 

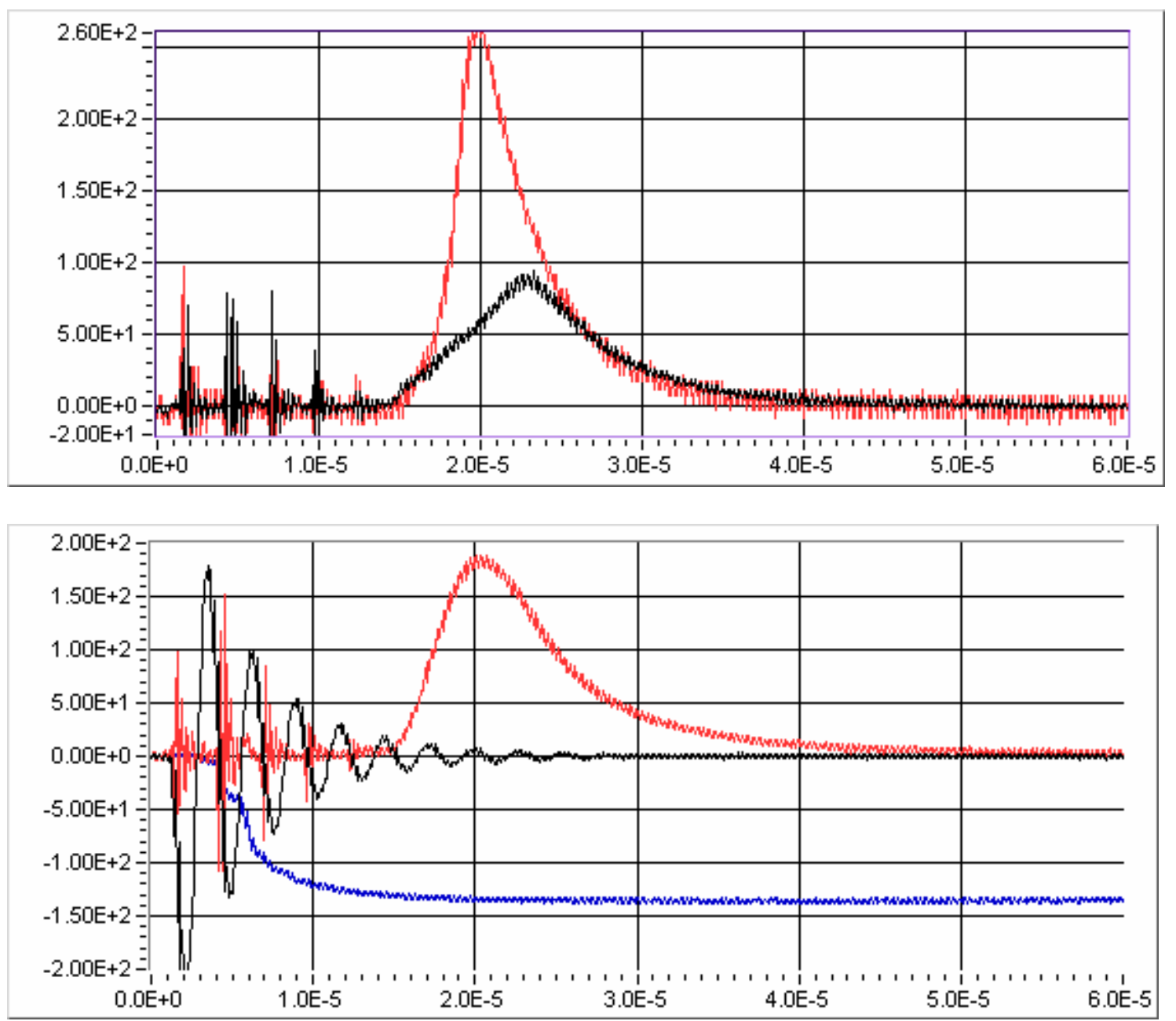

Figure 13 Superimposed data from a 14-shot sequence. Upper traces are the Faraday cup signals $\left(\mathrm{mA} / \mathrm{cm}^{2}\right)$ at $\mathrm{z}=16 \mathrm{~cm}$ and $\mathrm{r}=0$ and $2 \mathrm{~cm}$. Lower traces are driving current $(500$ A/div), PMT signal (arb. units) and Faraday cup $\left(\mathrm{mA} / \mathrm{cm}^{2}\right)$ at $\mathrm{z}=16 \mathrm{~cm}$ and $\mathrm{r}=4 \mathrm{~cm} .379$

\section{Azimuthal Uniformity}

The purpose of the Phase I uniformity measurements was to determine the extent of nonuniformity and to identify major sources contributing to non-uniformity. Optimizing the source in this regard will be undertaken in Phase II.

The azimuthal uniformity was measured using an array of Faraday cups that could be rotated about the axis. Measurements were made at 90 degree intervals. The peak Faraday cup signal and the integral of this signal for cups $16 \mathrm{~cm}$ from the source at 2 and $4 \mathrm{~cm}$ radii are plotted in Figure 14. As can be seen, there is a substantial azimuthal nonuniformity. The ratio of the maximum to minimum signal is $\sim 2.2$. This ratio decreases to 1.6 for measurements made $32 \mathrm{~cm}$ from the source. This non-uniform pattern is highly reproducible, at the same level as seen in Figure 13. 
Much of the non-uniformity is due to the performance of the high speed valve and the size of its plenum. Rotating the valve 90 degrees also shifts the angular location of the maximum and minimum signals, as can be seen in Figure 15.
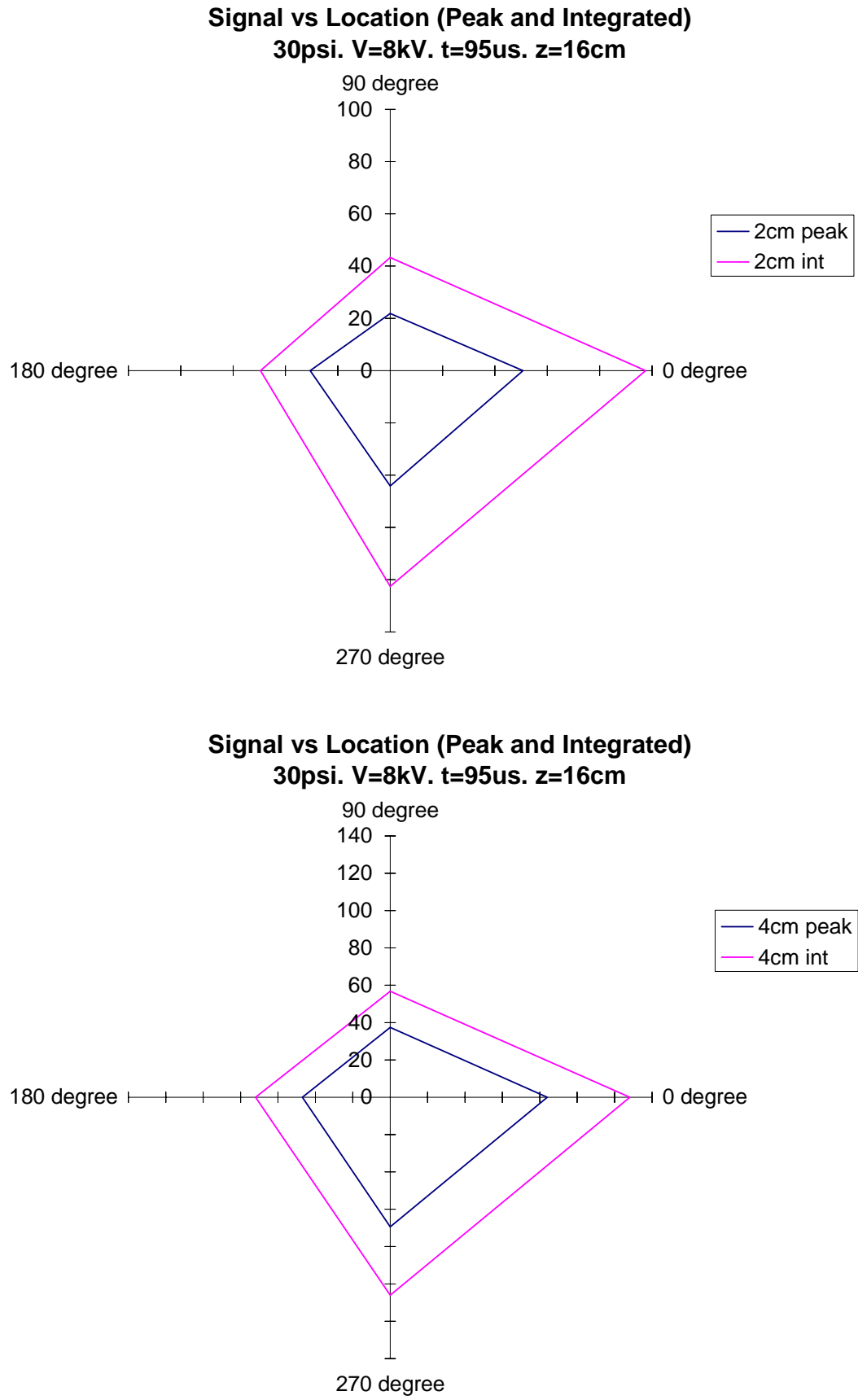

Figure 14. Azimuthal uniformity at radii of 2 and $4 \mathrm{~cm}$. Data measured at 4 angular locations. Peak and integrated values of the signal are plotted. 


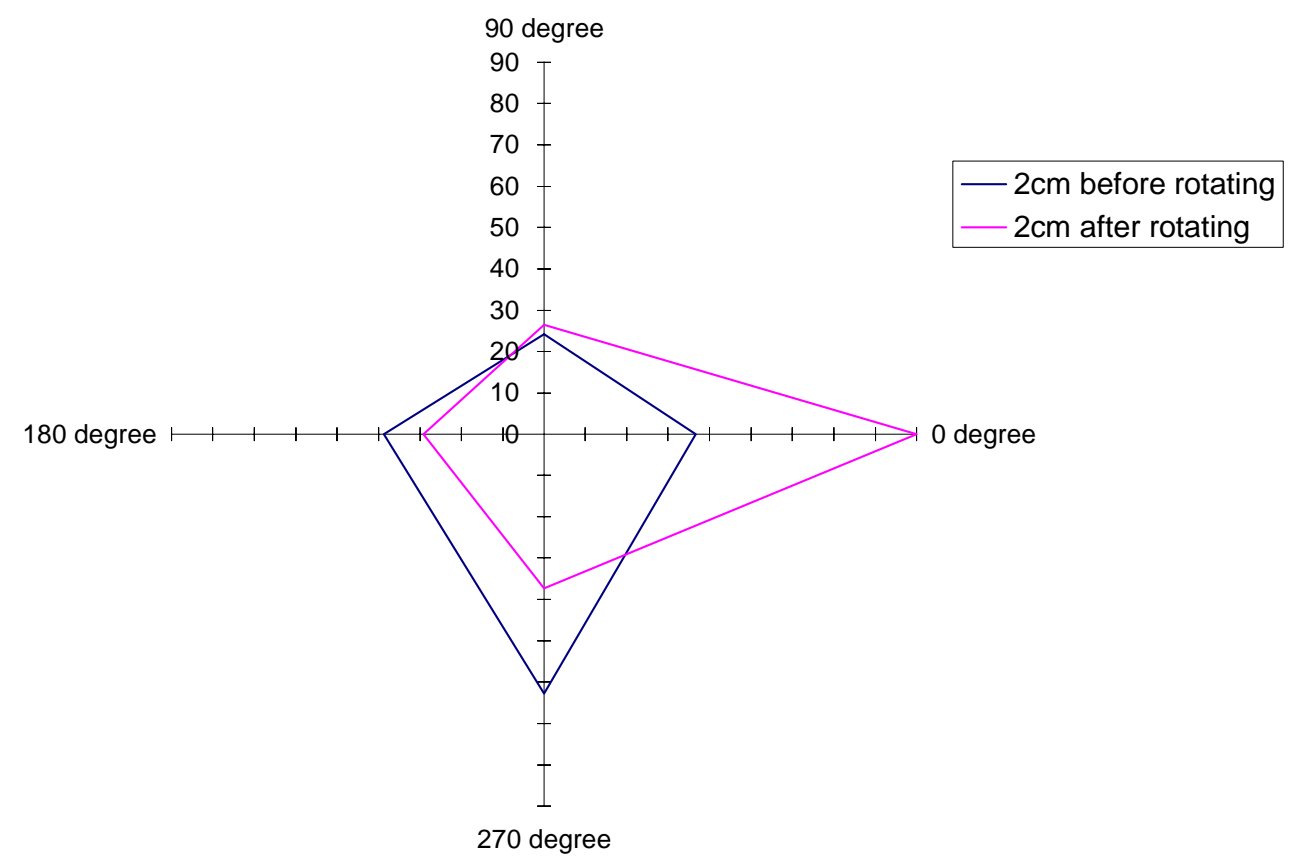

Figure 15. Effects of rotating the high speed valve 90 degrees counterclockwise. Data are peak signal size at $r=2 \mathrm{~cm}, z=16 \mathrm{~cm}$, expressed as a percentage of the center Faraday cup signal.

The degree of non-uniformity is increased by the manner in which we are using the valve. Fast ionization gauge measurements indicate a $5 \mu$ s spread in the opening time of the valve at different azimuthal locations. This has been observed with other valves in the past as well. As discussed earlier, the maximum ion flux occurs when the gas density is relatively low. At higher densities, collisions with the neutral gas prevent ions from leaving the source. Because of the relatively large plenum volume, this density is reached very near the beginning of the gas pulse. At this time, the ratio of the minimum and maximum density around the circumference of the valve is greatest because of the spread in opening time. Using a smaller plenum size would permit operation closer to the peak of the gas pulse, where this ratio is much smaller.

Changing the delay time between the high speed valve and ionization coil drivers demonstrates the effects of the changing gas density on ion flux output. Figure 16 shows the peak Faraday cup signals for different delay times at 4 different azimuthal locations. Also shown is the relative opening time of the gas valve at each of these locations. It can be seen that at early delay times the Faraday cup signals are greater at the angular positions ( 0 and 270) where the gas arrives first. The longer delay times results in the shift of the peak signals to the other angular locations (90 and 180) as gas begins to appear there, while at the previous locations the gas density has increased to the level that collisions with the ions begin to decrease the ion flux. 


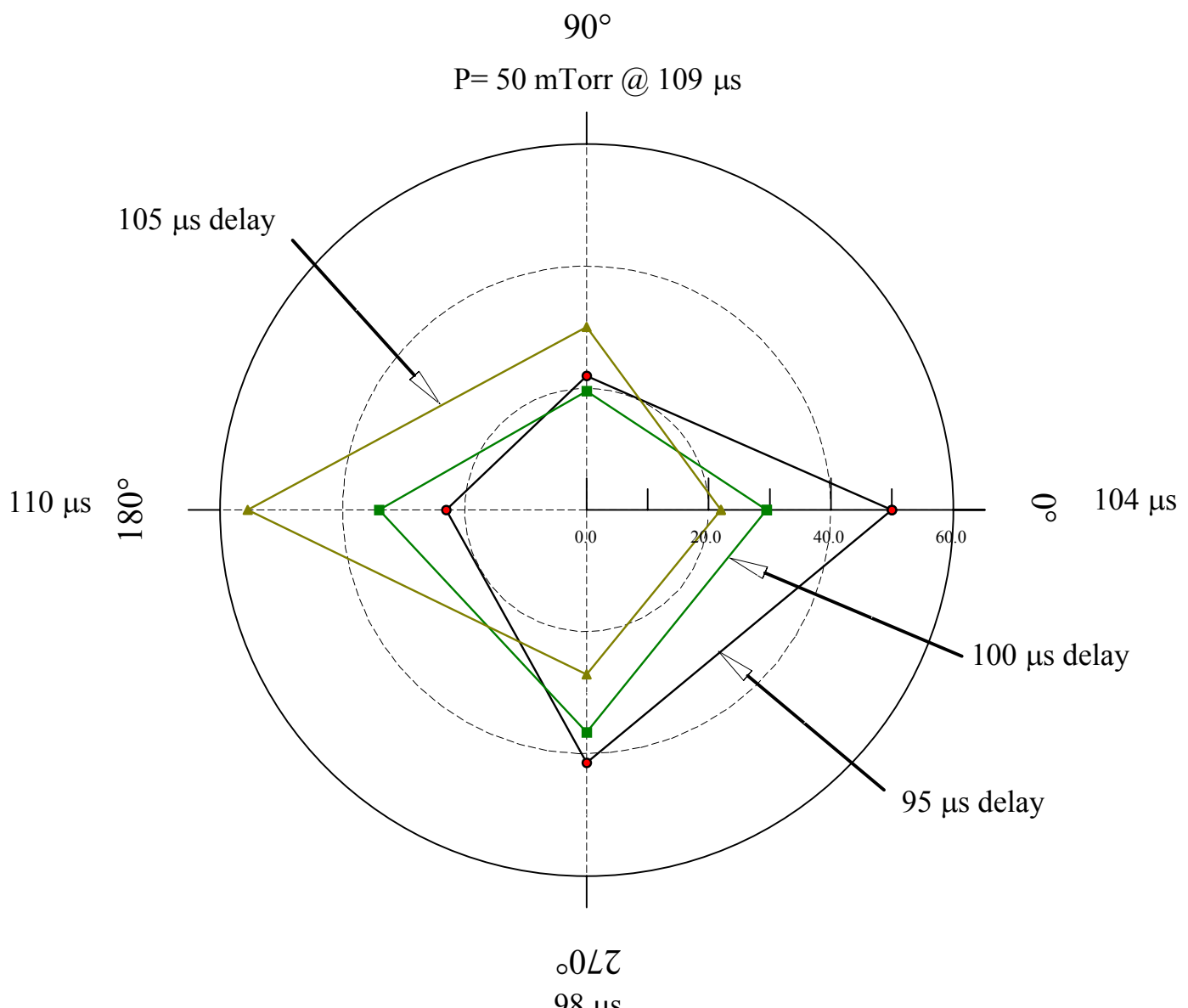

Figure 16. Relative peak Faraday cup signals for delay times of 95, 100 and $105 \mu$ s. The time interval from the triggering of the gas valve until the gas pressure has reached 50 $\mathrm{m}$ Torr at a radius of $4 \mathrm{~cm}$ is indicated for each 90 degree location.

\section{Radial Distribution}

The ion flux is a function of radius and distance $(8-28 \mathrm{~cm})$ from the source is shown in Figure 17. 
Applied Pulsed Power, Inc.

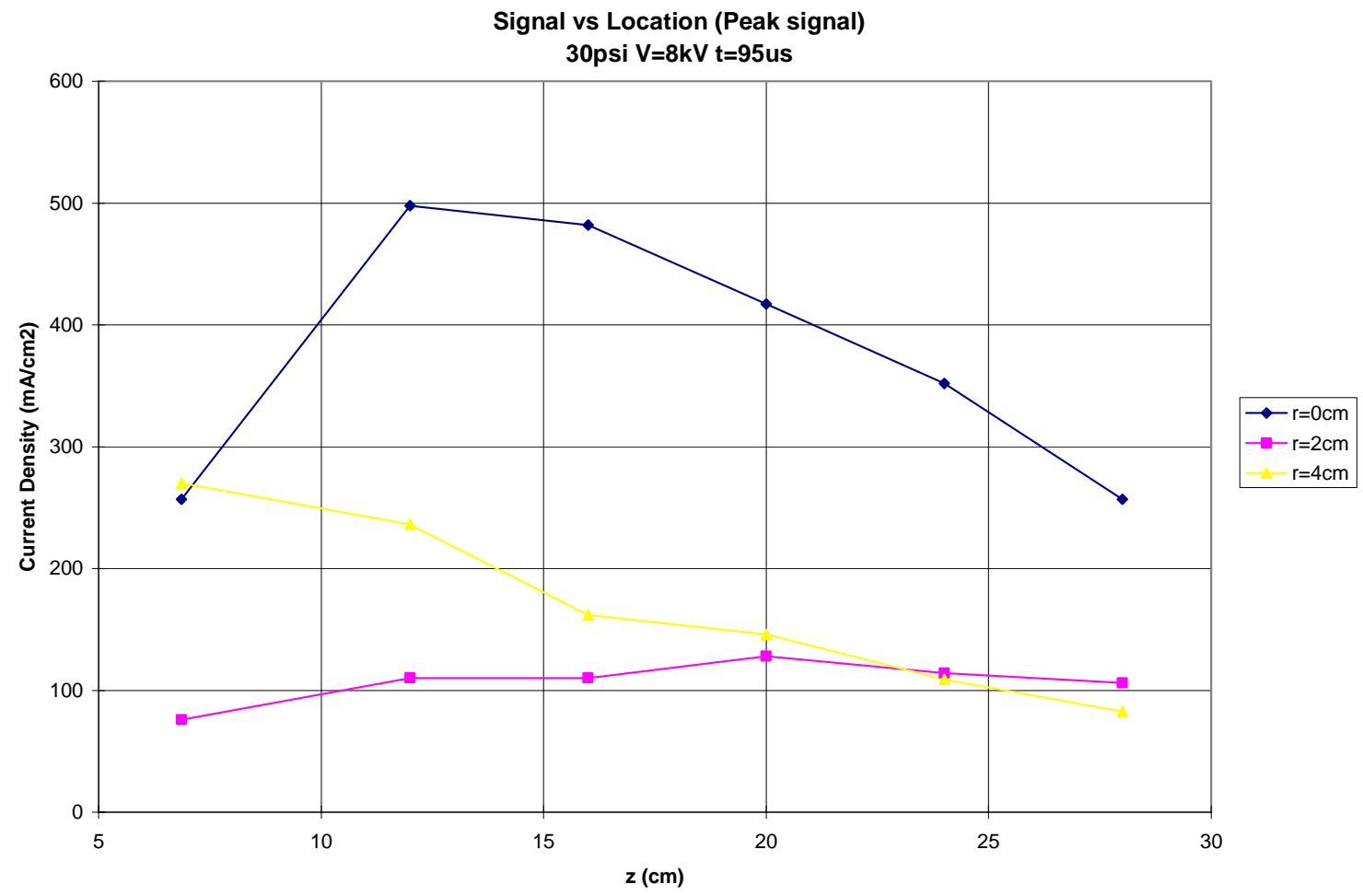

Signal vs Location (Integrated signal) 30psi $V=8 k V$ t=95us

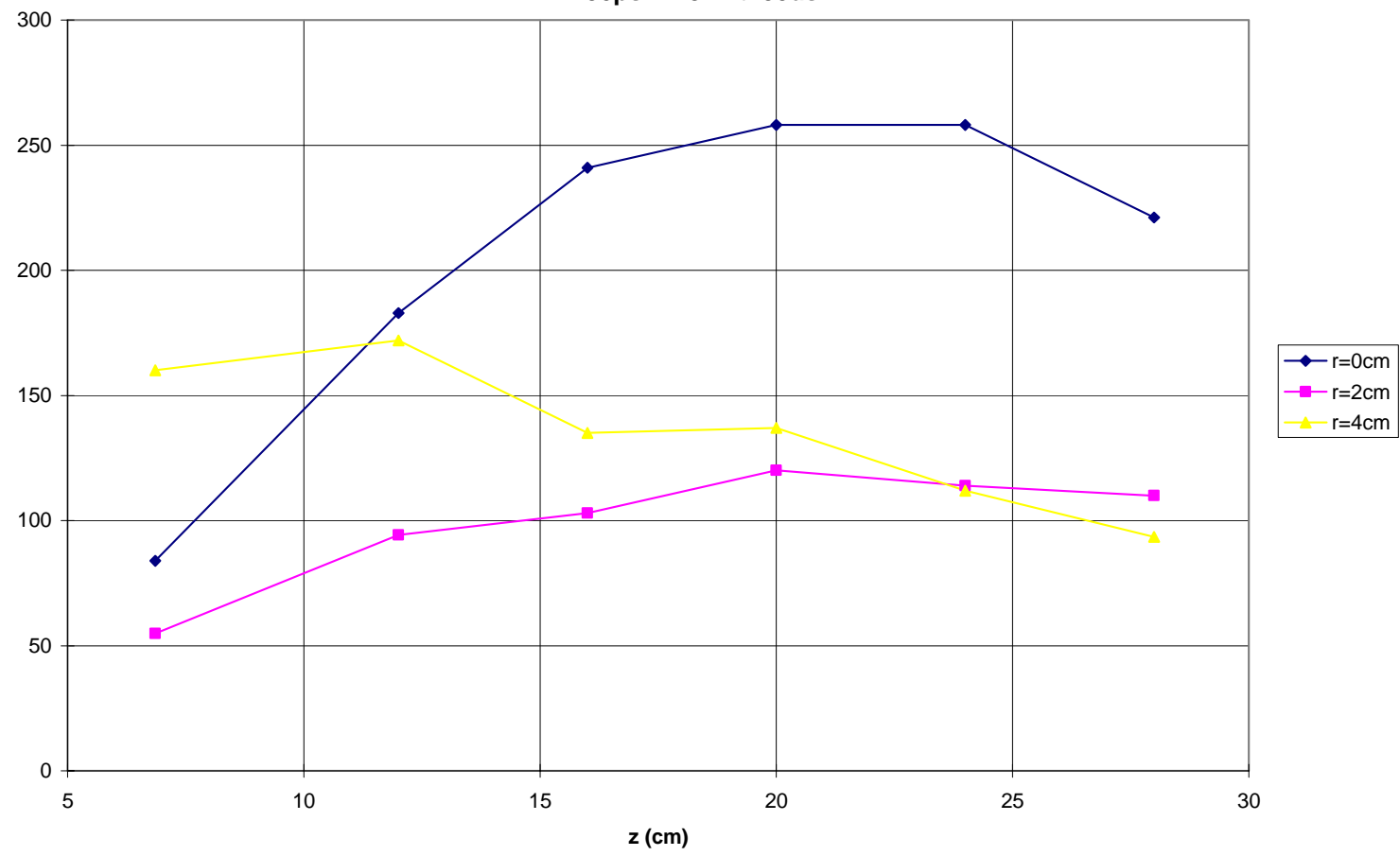

Figure 17. Radial ion flux distribution as function of distance from the source. 
The relative ion flux as a function of radius was also found to be dependent on the driving voltage. This can be seen in Figure 18 which compares Faraday cup measurements at 0 and $2 \mathrm{~cm}$ for different driver voltages. At $9 \mathrm{kV}$, the signals are very similar.

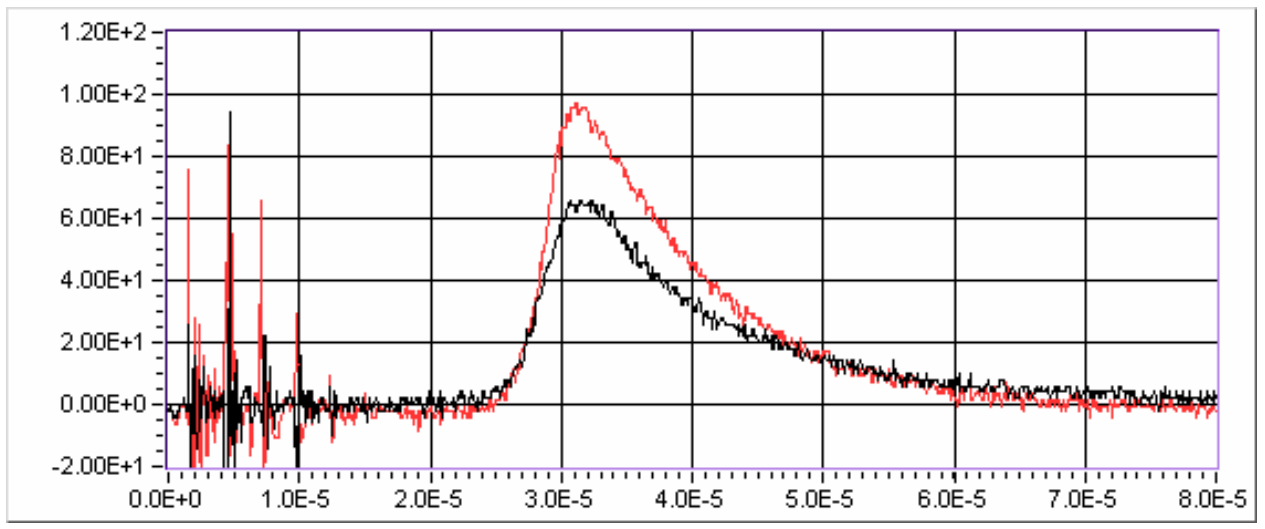

(a) $7 \mathrm{kV}$ driver voltage. 461

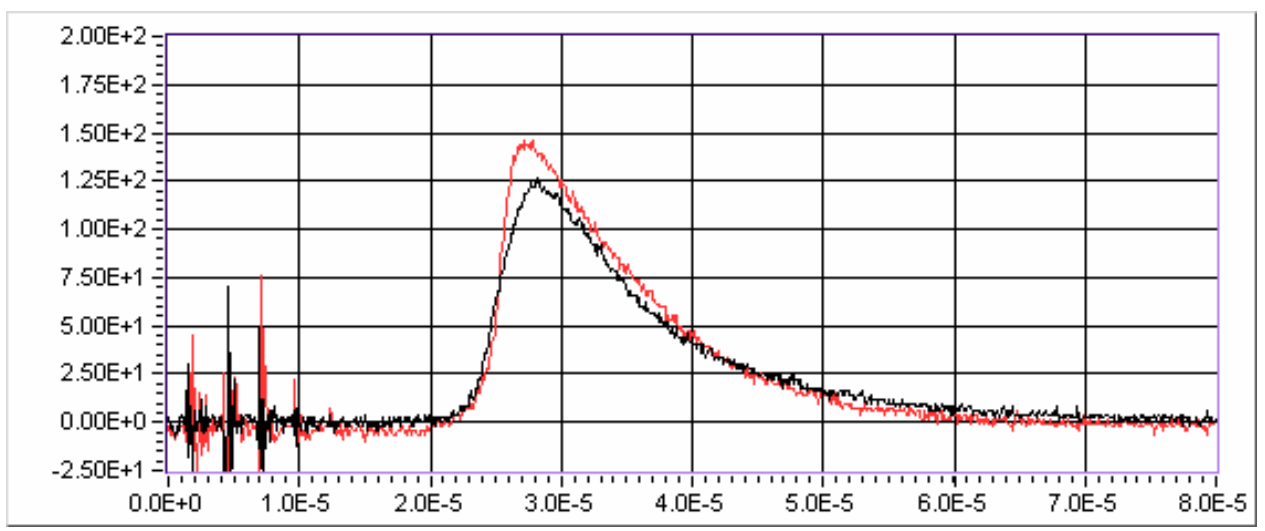

(b) $8 \mathrm{kV}$ driver voltage. 455

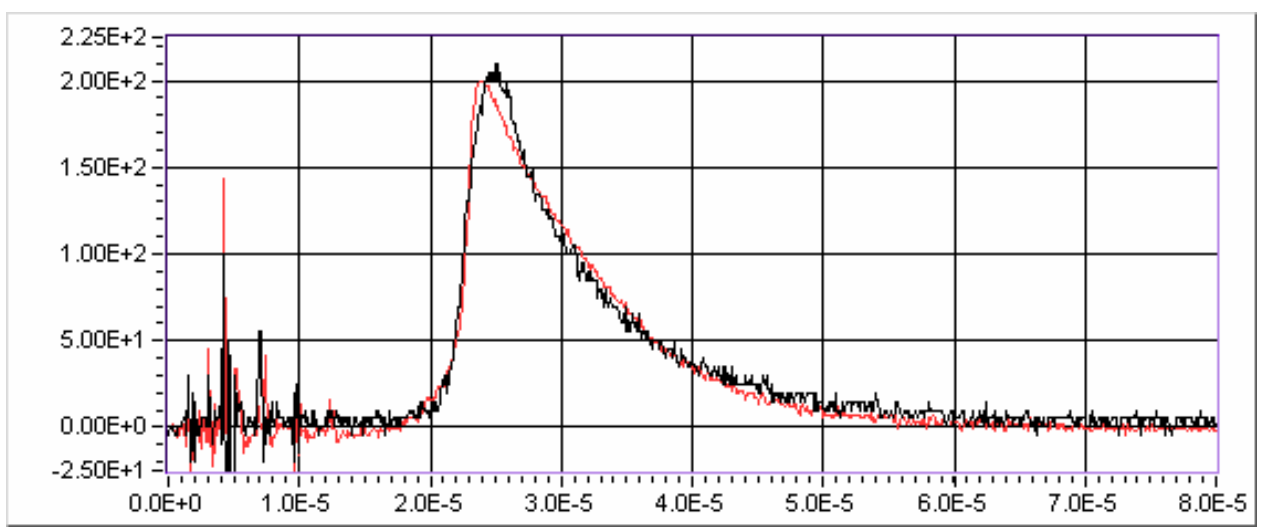

(c) $9 \mathrm{kV}$ driver voltage 462

Figure 18. Comparison of ion flux measured $32 \mathrm{~cm}$ from source at radii of 0 and $2 \mathrm{~cm}$ for different ionization coil driver voltages. 
It should also be possible to alter the magnetic field geometry of the source to adjust the radial distribution, but this was not attempted in Phase I.

\section{Effects of Driving Waveforms on Source Performance}

Experiments were undertaken to determine the effects of altering the driving wave form on the output of the source.

Initially, resistance was added to the circuit to increase the amount of damping. These results are shown in Figure 19. The effects of adding even a small amount $(0.17 \Omega)$ of damping are quite dramatic. The peak amplitude of the signal decreases by a factor of $>2$. The pulse shape is also affected, resulting in a flatter and somewhat longer pulse.

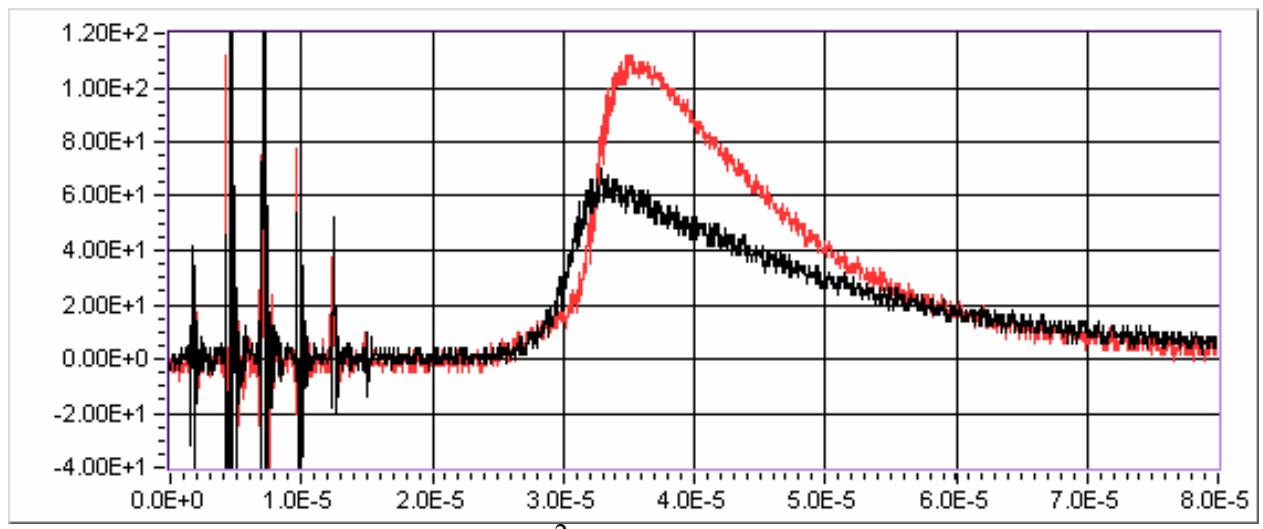

Faraday cups $\left(\mathrm{mA} / \mathrm{cm}^{2}\right)$ at $\mathrm{z}=32 \mathrm{~cm}, \mathrm{r}=0$ and $2 \mathrm{~cm}$.

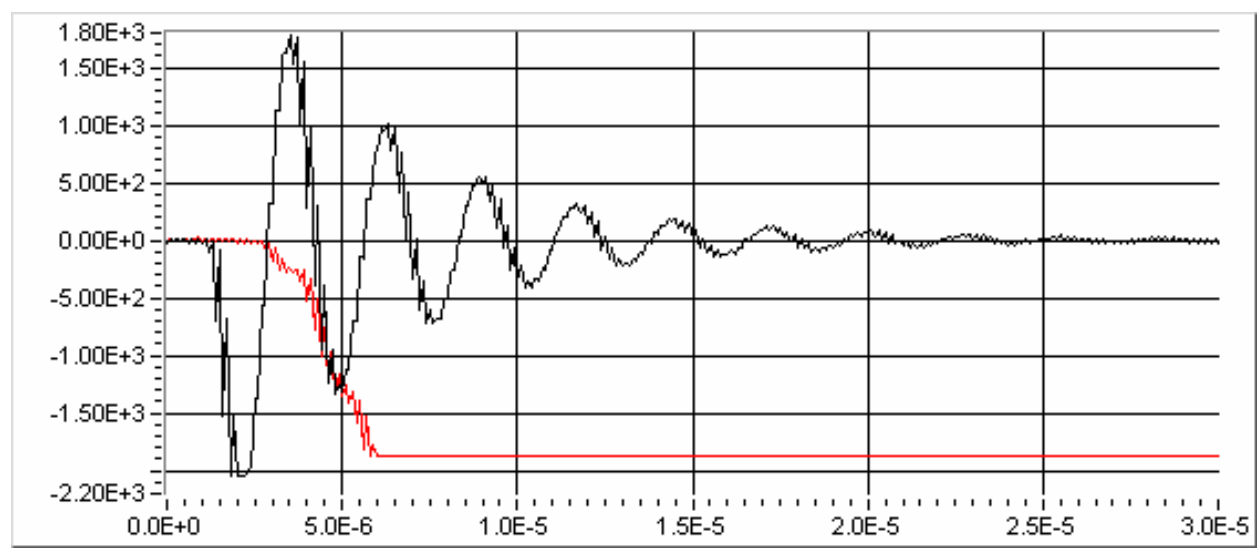

Driver current (A) and photomultiplier tube signal (x50)

Figure 19(a) No additional damping (envelope for 14 shots) 465 


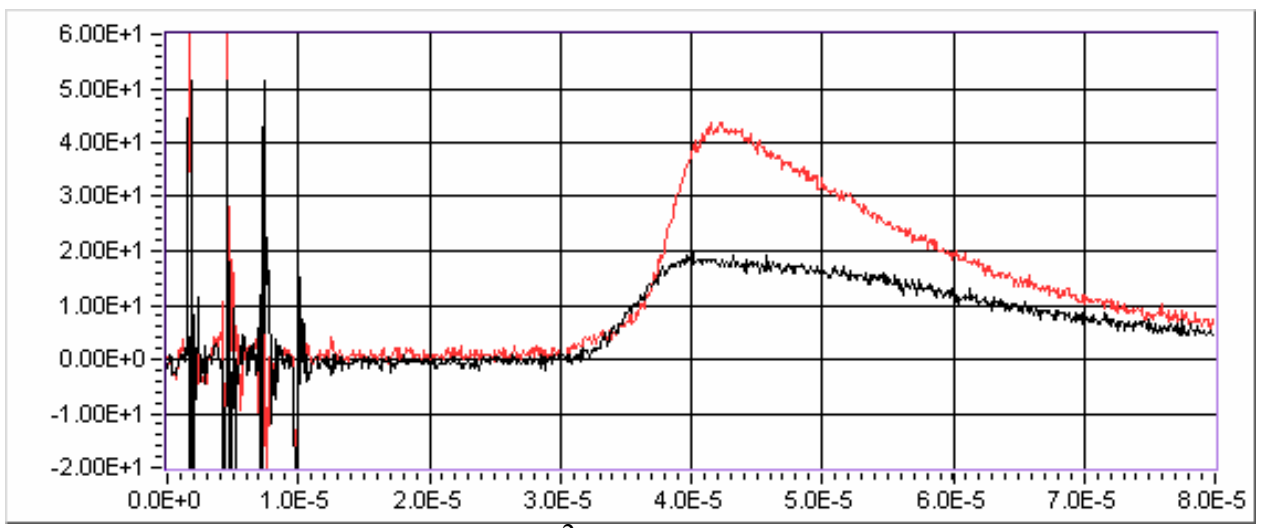

Faraday cups $\left(\mathrm{mA} / \mathrm{cm}^{2}\right)$ at $\mathrm{z}=32 \mathrm{~cm}, \mathrm{r}=0$ and $2 \mathrm{~cm}$.

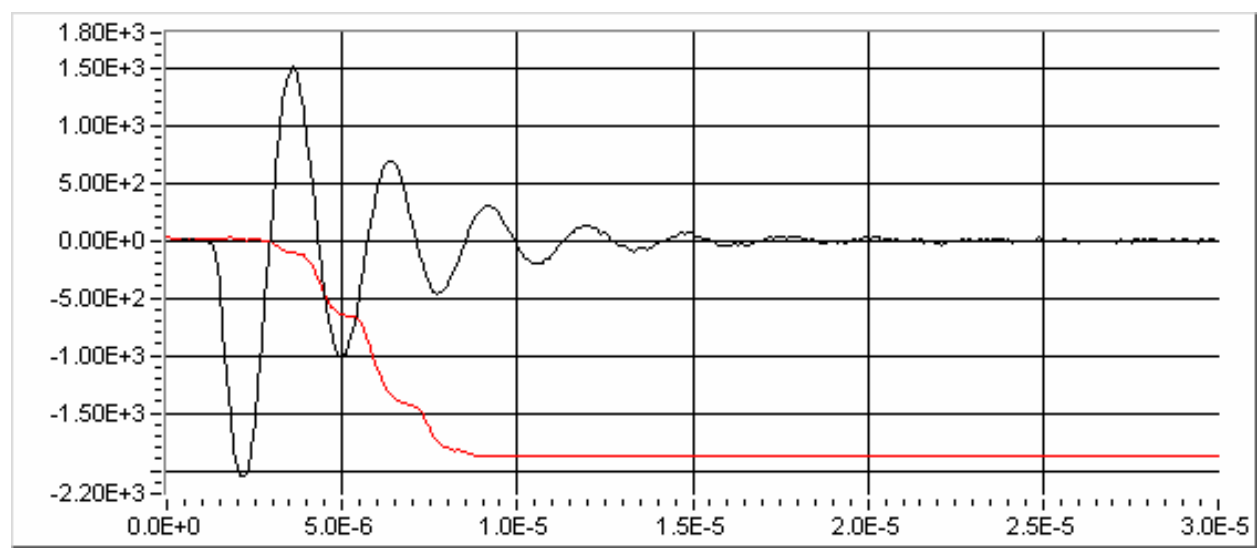

Driver current (A) and photomultiplier tube signal (x50)

(b) $0.17 \Omega$ damping 469

Figure 19. The effects of adding damping resistance to the ionization coil driver circuit on source output. All data with 30 psig argon and $8 \mathrm{kV}$ driver voltage.

To pursue this observation in a simple way, a second switch was added to truncate the drive pulse after a certain number of oscillations. Some of these results are shown in Figure 20. Truncating the pulses after 4 cycles, when the drive pulse has decayed to $15 \%$ of its initial value, decreases the output by $40 \%$. Truncating after 2 cycles decreases the signal by $85 \%$.

It appears that after the initial large amplitude cycle that results in the breakdown of the gas, a much smaller drive would be sufficient to generate and accelerate the ions. By tailoring the shape of the drive pulse, it may be possible to both extend the duration and control the shape and magnitude of the ion flux pulse. 
Applied Pulsed Power, Inc.

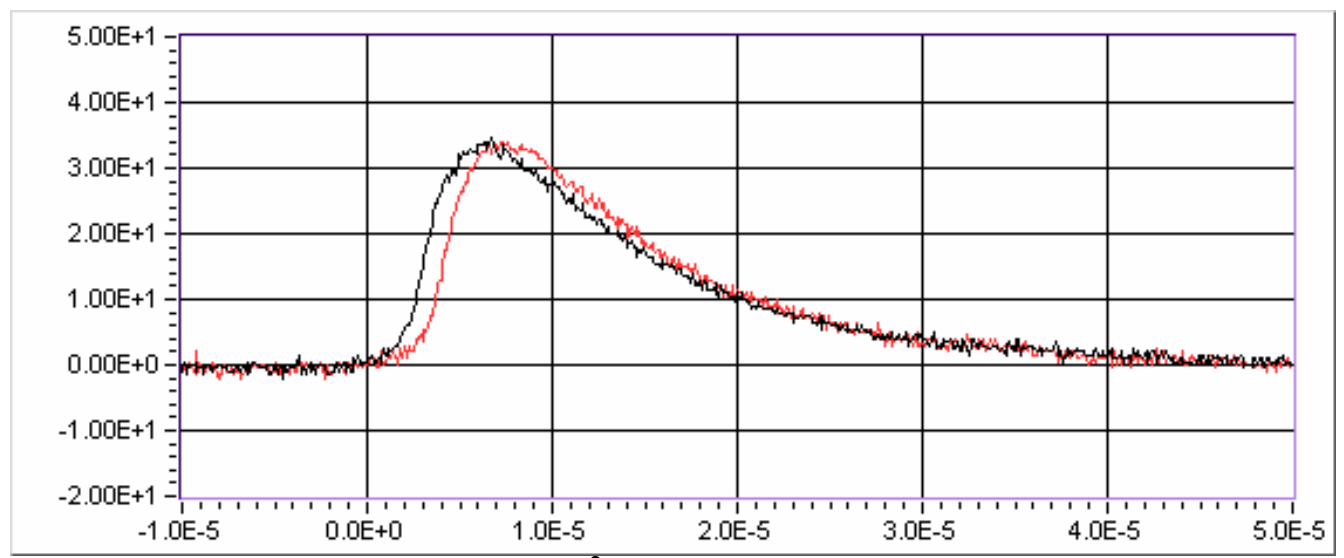

Faraday cups $\left(\mathrm{mA} / \mathrm{cm}^{2}\right)$ at $\mathrm{z}=32 \mathrm{~cm}, \mathrm{r}=0$ and $1.2 \mathrm{~cm}$.

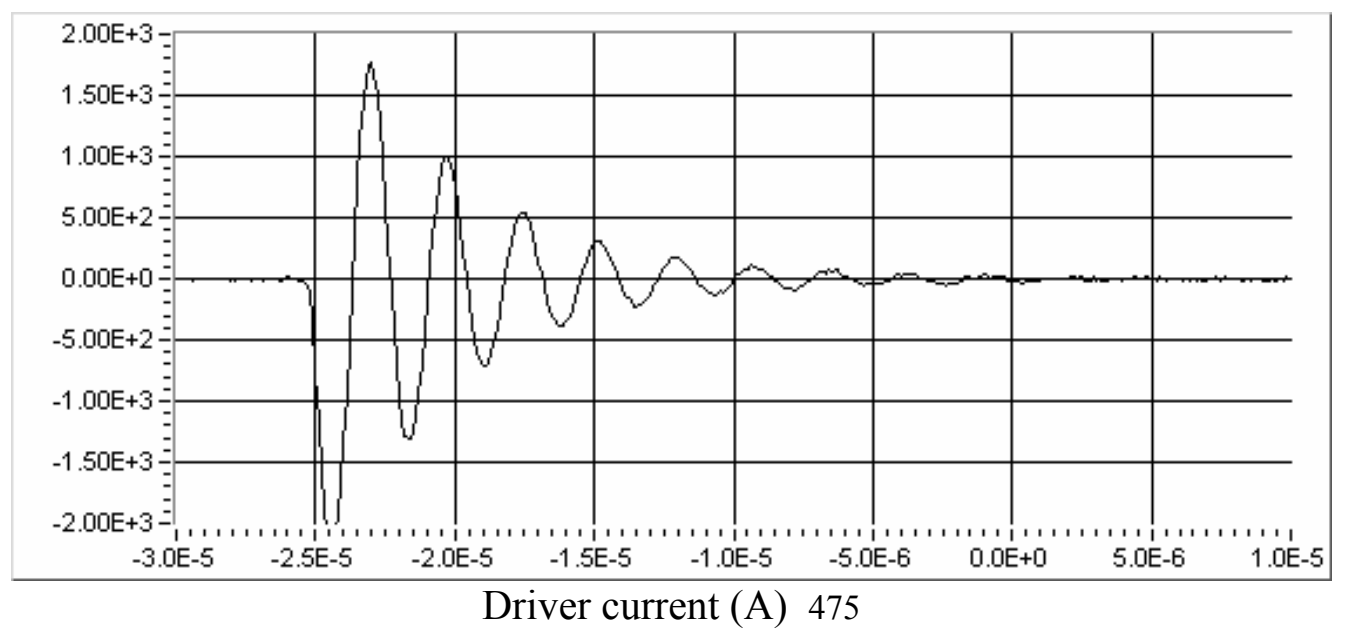

Figure 20(a) No truncation 
Applied Pulsed Power, Inc.
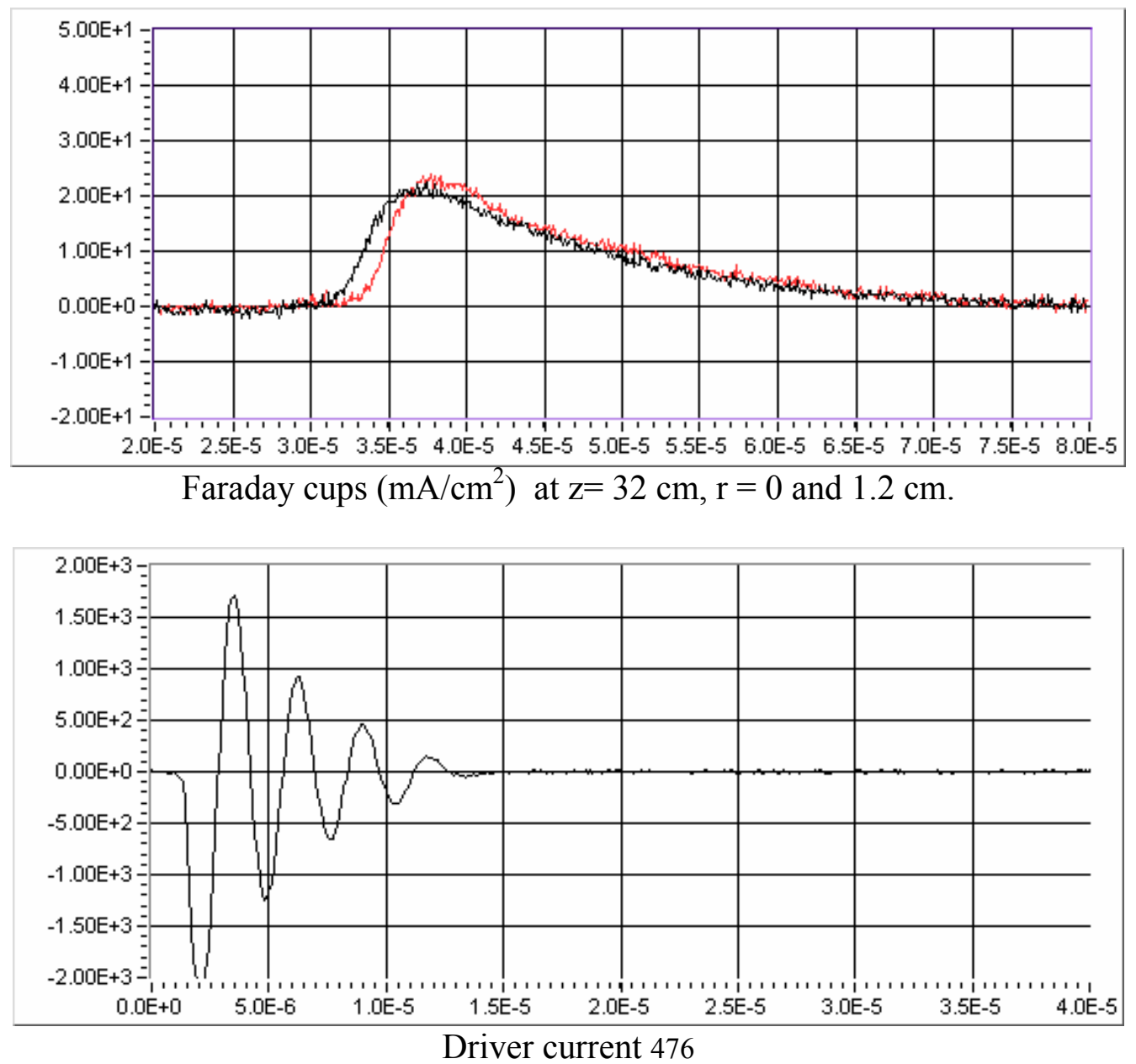

Figure 20(b) Truncated after $\sim 4$ cycles 


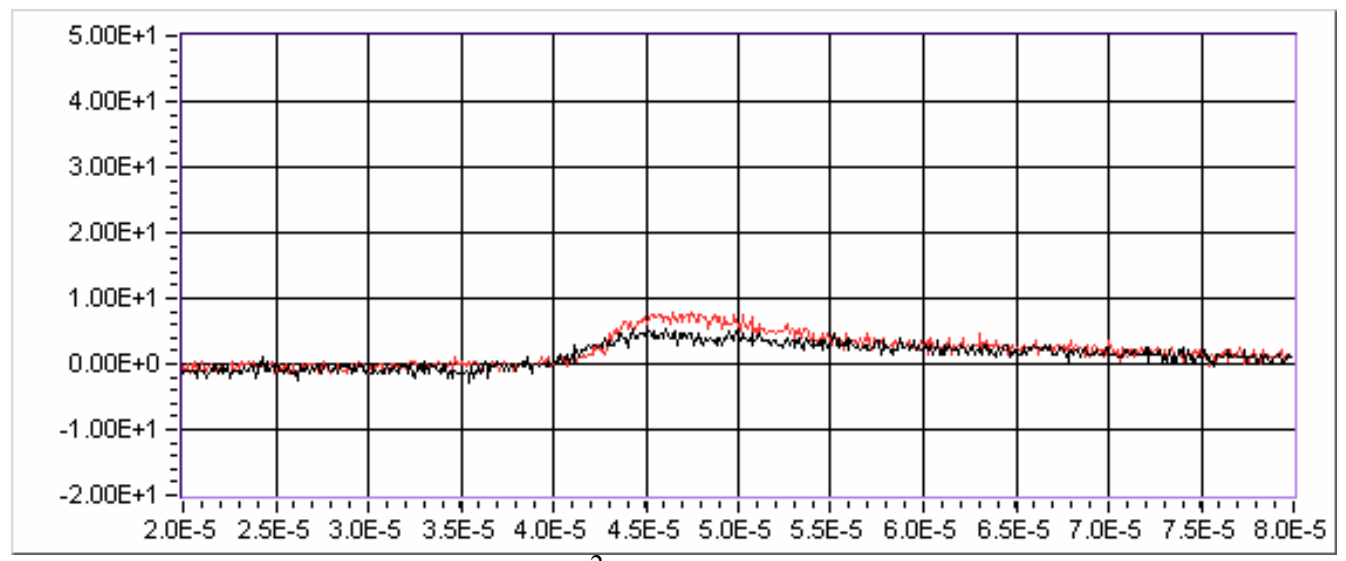

Faraday cups $\left(\mathrm{mA} / \mathrm{cm}^{2}\right)$ at $\mathrm{z}=32 \mathrm{~cm}, \mathrm{r}=0$ and $1.2 \mathrm{~cm}$.

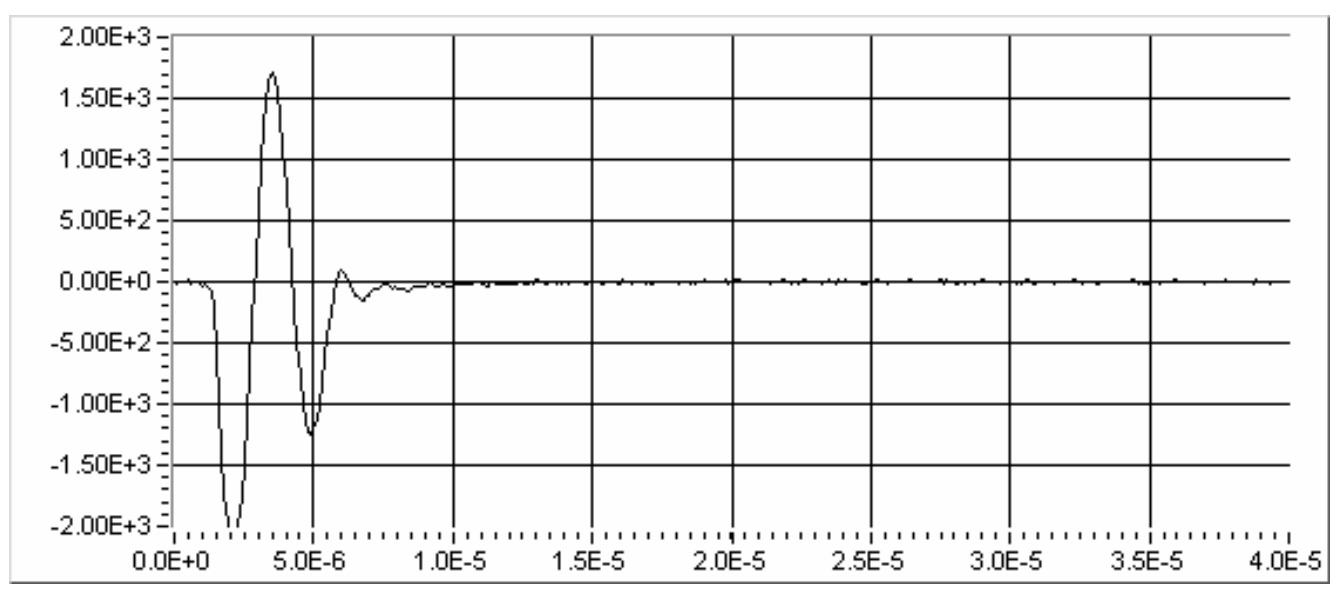

Driver current (A) 478

(c) Truncated after $\sim 2$ cycles

Figure 20. Effects of truncating the drive pulse on the output of the ion source. All data taken with 30 psig argon and $8 \mathrm{kV}$ driver voltage.

\section{Plenum Pressure}

Performance of the source was examined with high speed valve plenum pressures of 3, 1.6 , and 0.7 atm. Maximum source output was achieved with a longer coil-to-valve delay time at lower plenum pressures. Also, a large output was obtained over a longer span of delay times at lower pressures. Both of these observations are consistent with our belief that collisions with neutral gas molecules attenuate the directed ion flux at longer delay times.

The magnitude of the ion fluxes obtained at optimum delay times for different plenum pressures were comparable. It is clear therefore, that the plenum volume and thus the amount of gas loading for the system could be much smaller. 


\section{Charge State Measurements}

A pulsed time-of -flight (TOF) measurement was attempted to find the charge state distribution of the plasma ions. A pair of fine grids separated by $2 \mathrm{~mm}$ was used, with $0.5-2 \mathrm{kV}$ accelerating voltage applied. The TOF was measured to a Faraday cup $16 \mathrm{~cm}$ downstream. A TOF difference of about 1 microsec would result for $\mathrm{Ar}+2$ ions relative to $\mathrm{Ar}+1$. Unfortunately, the head of the plasma stream makes a smooth rise in about 5 microseconds, and just before this rise, noise from the driving circuit disturbs the baseline. Furthermore, plasma Debye shielding effects on the grids were clearly visible in the behavior of the signals with the accelerating voltage. The result is that this technique was able only to establish that doubly or higher charged species could not be more than roughly $10 \%$ of the plasma ion population. A more precise measurement will require gating of the plasma or the accelerating voltage to give a clear separation of the charge species, which could not be undertaken during Phase I.

\section{Discussion of Results Relative to Phase I Goals and the Requirements for Heavy Ion Fusion Accelerators}

The source we have investigated produces a stream of Argon plasma with approximately $80 \mathrm{eV}$ directed axial energy, and approximately $0.6 \mathrm{eV}$ transverse ion temperature. The ion current density is above $100 \mathrm{~mA} / \mathrm{cm}^{2}$, with different levels available at different operating conditions. The ion density is thus $\sim 3 \times 10^{13} / \mathrm{cm}^{3}$. The source has produced fairly uniform ion current density into a $4 \mathrm{~cm}$ diameter circle, and has total pulse length $>10$ microseconds.

Two features of the data perhaps stand out relative to the stringent requirements for a HIF ion source. First is that the inductive source shows very reproducible performance under a wide variety of operating conditions. This is the result of the statistics of the gas breakdown as driven by the inductive electric field, and using the preionization method we have described. The level of this reproducibility remains to be accurately determined, since it is better than the present diagnostics can resolve at the 5\% level.

Second, several means have been identified with the potential to control and optimize both the radial and temporal uniformity of the plasma stream from this source. Though the present version of the source has substantial spatial non-uniformity, and a peaked temporal pulse shape; since the spatial and temporal shapes are highly reproducible, these means of control can really be used in practice. During the brief Phase I investigation it has been observed that simple changes in driving waveform can improve both radial uniformity and temporal shape. These observations give promise that with careful choice of driving pulse shape and driving coil configuration, both radial uniformity and temporal pulse shape can be adjusted to meet the requirements for HIF. This optimization is a prime objective of Phase II. 
Clearly, the azimuthal non-uniformity observed in the present source is caused by a nonuniform supply of gas from the fast valve to the ionization region. There are two possible means of reducing this non-uniformity. One is to determine the causes of the differences in opening times around the circumference of the valve, and then control the manufacture and mechanical properties of the valve and its beryllium-copper diaphragm to minimize these differences. The second option is to use a different gas flow design. In this concept, gas would be supplied by the valve to a secondary plenum. The exit of this plenum would be an axial tube-shaped supersonic nozzle. This axial flow would then impinge on a nozzle piece that would bend and redirect it radially outward. Thus the flow from the valve would be mixed in the secondary plenum, and the final azimuthal uniformity of the flow would be determined only by the mechanical tolerance of the nozzle, which is easy to fabricate accurately. It is highly advantageous to retain essentially the present gas valve design, since this valve has been proven to give long lifetime, operates at more than adequate repetition rate for HIF application, and is reproducible in its opening down to the one microsecond timescale, giving highly reproducible gas output.

The results of the Phase I studies allow comparison of the pulsed, streaming source with other sources that have been investigated for HIF. In comparison with the $\sim 0.1 \mathrm{eV}$ surface ionization sources presently used, our source provides an order of magnitude higher current density and reduced gas load; also the gas produced is argon instead of potassium, which is more benign in its effects on the accelerator structure. Surface ionization sources are not capable of reaching the high current density $\left(>100 \mathrm{~mA} / \mathrm{cm}^{2}\right)$ regime that enables use of a more compact injector structure. The pulsed streaming source clearly meets this critierion. The transverse ion temperature of $\sim 0.6 \mathrm{eV}$ is higher than for the surface sources, but this is tolerable at the higher current density ${ }^{3}$.

The comparison with RF sources in terms of ion optics for beam extraction and acceleration is discussed below. It should be noted that the risetime of the pulsed streaming source is somewhat faster than thermal RF sources, which may allow better control of the head of the extracted beam pulse. The gas load is smaller because of the pulsed gas valve of the streaming source, which eases pumping requirements and allows lower pressure in the injector optics, reducing charge exchange and other deleterious effects on the beam.

The true capabilities of the source for the HIF application can only be established by extracting a beam from the plasma because the brightness and emittance attainable in the beam extracted from a plasma ion source depend sensitively upon the level of control that can be attained of the plasma surface from which the ions are accelerated. This plasma "meniscus" forms at the entrance to the extraction electrode structure as the result of the self-consistent development of the balance between plasma ion flux supplied and beam ion current extracted. The effective "surface" of the plasma where the electric field goes to zero acts as an electrostatic boundary for the extraction optics. Its shape is an element in the design of the ion optics, and so the true test of the uniformity and reproducibility of a plasma ion source is the steadiness of this shape during an extraction pulse. 
With a purely thermal plasma source, this surface develops basically as a Bohm sheath, and in steady state, ion flux arrives at the surface at the ion sound speed. The design of extraction optics including the self-consistent shape of the plasma meniscus is a difficult problem, even assuming a "perfect" (uniform) plasma, because it involves the full plasma physics of the sheath in addition to the calculation of the extracted ion orbits.

Sophisticated codes have been developed to attack this design problem, but use of these tools was beyond the scope of Phase I of this program. However, ion beam extraction from plasmas has been developed over several decades, for many accelerator applications including energetic neutral beams for magnetic fusion and industrial accelerators, and analytic and phenomenological models have been developed based on this experience. During Phase I, study of this background led to the conclusion that the present source would operate somewhat differently from typical thermal sources. The ion flux in the axially streaming plasma is far in excess of the sound speed. In comparison with a thermal plasma source, for a given extracted beam current density, this streaming source would have a high ion velocity and low density at the plasma meniscus, versus a low velocity and high density for a cold thermal source. The difference compared with a typical sub-eV RF source is about an order of magnitude; the velocity is ten times higher and the density is ten times lower for the pulsed streaming source.

There are at least two possible implications of this difference. First, it would appear that the higher density of the thermal source is an advantage in the following sense: a fluctuation giving imbalance between supply and extraction would be compensated for by movement of the plasma meniscus as ions were eroded from or accumulated there. At a given current density, that movement of the meniscus would therefore occur more slowly with higher plasma density, so the extraction optics would be better preserved against small perturbations. However, to keep the shape of the plasma meniscus and the extraction optics constant, the basic parameter that must be held constant with the streaming source is the streaming ion flux density, whereas for the thermal source it is the combination of ion density and electron temperature (which determine the sound speed). The relative difficulty in attaining the required level of control is thus not easily quantified in comparing the two regimes. Each must be determined from its own performance.

The second implication of this difference involves the design of the extraction optics. Since the ions from the streaming source enter the extraction electrode with $\sim 80 \mathrm{eV}$ directed energy, their space charge density in the electric field of the extraction optics is initially an order of magnitude lower than for the same current density of thermal, sub-eV ions. This comparison certainly favors the streaming source in the attainment of high brightness through the extraction optics, but the difference may be small since this initial condition affects only a small distance into the accelerating field. Quantitative analysis of this effect awaits the use of a code such as IGUN or PBGUN, as proposed by our LBNL collaborators for the Phase II proposal. 


\section{References}

1. "Pulsed Inductive Plasma Source Development for Advanced Propulsion Systems", S. C. Glidden, J. B. Greenly, M. Richter, Final Report, NASA Cont. NAS99052, June 1999.

2. "Thermal surface treatment using intense, pulsed ion beams," Mat. Res. Symp. Proc. 316 (1994) 521 (with R.W. Stinnett, R.G. Buchheit, F.A. Gruelich, C.R.Hills, A.C. Kilgon, D.C. McIntyre, M.O. Thompson, G.P. Johnston and D.J. Rej).

3. J.W. Kwan et al., "Ion sources and injectors for HIF induction linacs", Nuclear Instruments and Methods in Physics Research A 464 (2001) 379-387 


\section{List of Figures}

Figure1. Block diagram of the plasma source. $\quad 4$

$\begin{array}{lll}\text { Figure 2. } & \text { Axial gas flow ion source. } & 6\end{array}$

Figure 3. Ionization coil driver, photomultiplier tube and ion current 7 density waveforms.

Figure 4. Radial gas flow source. $\quad 9$

Figure 5. Collimated aperture Faraday cup. $\quad 10$

Figure 6. Relative peak ion flux as a function of the time delay between $\quad 11$ the valve and ionization coil drivers.

Figure 7. Fast ionization gauge measurements of the gas pressure. 12

Figure 8. Axial gas flow source performance for coils with closely and 13 widely spaced turns.

Figure 9. Signals from Faraday cups separated by $8 \mathrm{~cm}$. 14

$\begin{array}{ll}\text { Figure 10. Angular divergence measurements. } & 17\end{array}$

Figure 11. Ion flux for 10 and 20 degree nozzles at different delay times. 18

Figure 12. Ion flux $16 \mathrm{~cm}$ from the source as a function of driver voltage. 19

Figure 13. Superimposed data from a 14-shot sequence. 20

Figure 14. Azimuthal uniformity at radii of 2 and $4 \mathrm{~cm}$. 21

Figure 15. Effects of rotating the high speed valve 90 degrees counterclockwise. 22

Figure 16. Relative Faraday cup signals for delay times of 95, 100 and $105 \mu$ s. 23

Figure 17. Radial ion flux distribution as function of distance from the source. 24

Figure 18. Comparison of ion flux for different ionization coil driver voltages. 25

Figure 19. The effects on source output of adding damping resistance to the $\quad 26,27$ ionization coil driver circuit.

Figure 20. Effects of truncating the drive pulse on the output of the ion source. 28-30 
Applied Pulsed Power, Inc.

Appendix B

DPP03 Presentation 


\title{
Inductive Plasma Ion Source for Heavy Ion Accelerators
}

\author{
Howard D. Sanders ${ }^{*}$, Steven C. Glidden \\ Applied Pulsed Power, Inc.
}

Daniel L. Dongwoo, John B. Greenly

Laboratory for Plasma Studies

Cornell University

American Physics Society

$45^{\text {th }}$ Annual Meeting of the Department of Plasma Physics

October $31^{\text {st }}, 2003$

Albuquerque, New Mexico

\begin{abstract}
A pulsed inductive gas breakdown plasma source is being developed as an ion source for heavy ion accelerators. A fast pulse gas valve introduces a localized Argon puff in the region in front of a pulsed coil, which induces a voltage breaking down the gas and driving the plasma. This results in highly directed streaming plasma of ion flux density up to $\sim 1 \mathrm{~A} / \mathrm{cm}^{2}$. Preliminary investigations determined that this source might satisfy the requirements for heavy ion fusion accelerators. Recent results on ion flux density, transverse emittance, and especially methods for controlling the temporal pulse shape will be described.
\end{abstract}

Work sponsored by DoE Grant DE-FG02-01ER83147 


\section{Inductive Plasma Ion Source for Heavy Ion Accelerators}

A pulsed Inductive gas Breakdown plasma Source (IBS) is being developed as an ion source for heavy ion accelerators. Section 1 shows diagrams and pictures of the source. A fast pulsed gas valve introduces a localized Argon puff in the region in front of a driving coil. A fast-rising current pulse applied to this coil induces an electric field that breaks down the gas. The initiation and reproducibility of the breakdown is enhanced by electrons emitted from a spark gap in a coil inductively coupled to the driving coil. The resulting plasma is accelerated away from the driving coil by the JxB force. This inductively coupled driving force results in highly directed streaming plasma of ion flux density $>1 \mathrm{~A} / \mathrm{cm}^{2}$.

Preliminary investigations (SBIR, phase I) determined that this type of source might satisfy the requirements for heavy ion fusion accelerators. The ion flux density is large enough that the source can be located $\sim 50 \mathrm{~cm}$ or more from the injection point, allowing for collimation to select a very lowemittance ion population. Section 2 discusses recent results including ion flux density and transverse emittance, and methods for controlling the temporal pulse shape.

The pulse shape requirement may be the most difficult challenge for this type of source to meet. At 30-50 cm downstream of the source region, the rise-time of the ion pulse is slow and the pulse is smoothly peaked in time rather than constant over some desired interval. Section 3 shows examples of the varying driving conditions, including driving current waveform and coil configuration, which failed to approach the required fast rise-time and flat top for the ion current.

We have recently invented a new technique to address this limitation. A second pulsed coil, mounted downstream of the source, can strongly affect the intensity of the transmitted ion flux. Preliminary data shows that the flow can be almost entirely suppressed by this "modulator" coil. Also, if the modulator current is quickly turned off, a fast (sub- $\mu$ s) rise-time may be created downstream. This is illustrated in the figures included in section 2 . The shape of the emitted flux is variable depending on the shape and magnitude of the current in the modulator coil. These effects are certainly 
caused by deflection of the plasma stream by the magnetic field produced by the coil current.

We are continuing the investigation into the characteristics of this source. Section 4 includes pictures taken with our recently acquired ICCD camera using a gate of $3 \mathrm{~ns}$. More research into the design of the source and modulator coil is required to produce an ion beam suitable for heavy ion accelerators. 


\section{Inductive Plasma Ion Source for Heavy Ion Accelerators}

\section{Section 1: Design of Source}

- Diagram 1: Section View of Source and Faraday Cup

- Diagram 2: Drawing of Source Including Dimensions

- Picture 1: View of Source from Front of Chamber

- Picture 2: View of Chamber

\section{Section 2: Characteristic Ion Current Data}

Effect of Modulation on Ion Current

- Modulation is the use of a set of coils in front of the source which can control the transmittance of the ion current

- Figure 1: Effect of Power of Modulation on Ion Current

- Figure 2: Effect of Time of Modulation on Ion Current Divergence of Ion Current

- Figure 3: Normalized Peak Ion Current versus Faraday Cup Angle for Selected Radii at $22.3 \mathrm{~cm}$ from Source

- Figure 4: Normalized Peak Ion Current versus Faraday Cup Angle for Selected Radii at $32.3 \mathrm{~cm}$ from Source

- Figure 5: Peak Ion Current versus Faraday Cup Angle for Selected Radii at $22.3 \mathrm{~cm}$ from Source

- Figure 6: Peak Ion Current versus Faraday Cup Angle for Selected Radii at $32.3 \mathrm{~cm}$ from Source

- Table 1: Single Standard Deviation Divergence Calculated Using Specified Gaussian Width

- Table 2: Observed Angle of Peak Ion Current versus Radius Effect of Modulation on Divergence of Ion Current

- Figure 7: Normalized Peak Ion Current versus Faraday Cup Angle for Selected Radii at $22.3 \mathrm{~cm}$ from Modulated Source

- Figure 8: Peak Ion Current versus Faraday Cup Angle for Selected Radii at $22.3 \mathrm{~cm}$ from Modulated Source

- Table 3: Single Standard Deviation Divergence Calculated Using Specified Gaussian Width (modulated versus unmodulated)

- Table 4: Observed Angle of Peak Ion Current versus Radius (modulated versus un-modulated) 


\section{Section 2: Characteristic Ion Current Data (continued)}

\section{Time of Flight}

- Figure 9: Ion Current versus Time at Different Distances from Source

- Table 5: Measured Time to Specified Ion Current Level

- Table 6: Calculated Velocity for Specified Ion Current Level

- Table 7: Calculated Time of Origin of Ion Current Using Time of Flight Data

\section{Section 3: Attempts to Alter Ion Current Profile Using Different Source Configurations}

- Several different methods were attempted to alter the ion current profile including changing the timing between the gas puff and the driving current, the density of the gas in the valve, the opening of the valve, the position and inductance of the driving coils, the waveform of the pulsed power, as well as other methods.

- Figure 10: Driving Current versus Time for Different Source Configurations

- Figure 11: Ion Current versus Time for Different Source Configurations

\section{Section 4: ICCD Pictures of the Source Plasma}

Front View Pictures

- Figure 12: Timing of Front View Pictures Relative to Driving and Ion Currents

- Picture 3 through Picture 10: the red circles represent the driving coil locations

Side View Pictures

- Figure 13: Timing of Side View Pictures Relative to Driving and Ion Currents

- Picture 11 through Picture 18: the red lines represent the plane of the driving coils and the perpendicular to that plane along the edge of the nozzle 
Diagram 1: Section View of Source and Faraday Cup

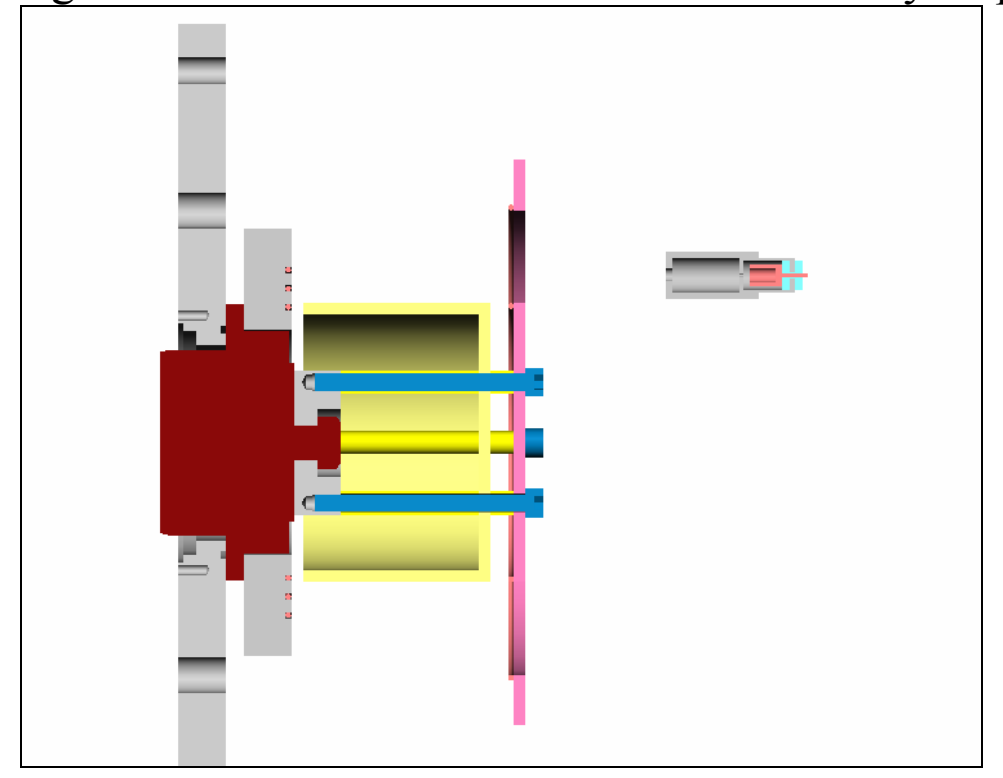

Diagram 2: Drawing of Source Including Dimensions

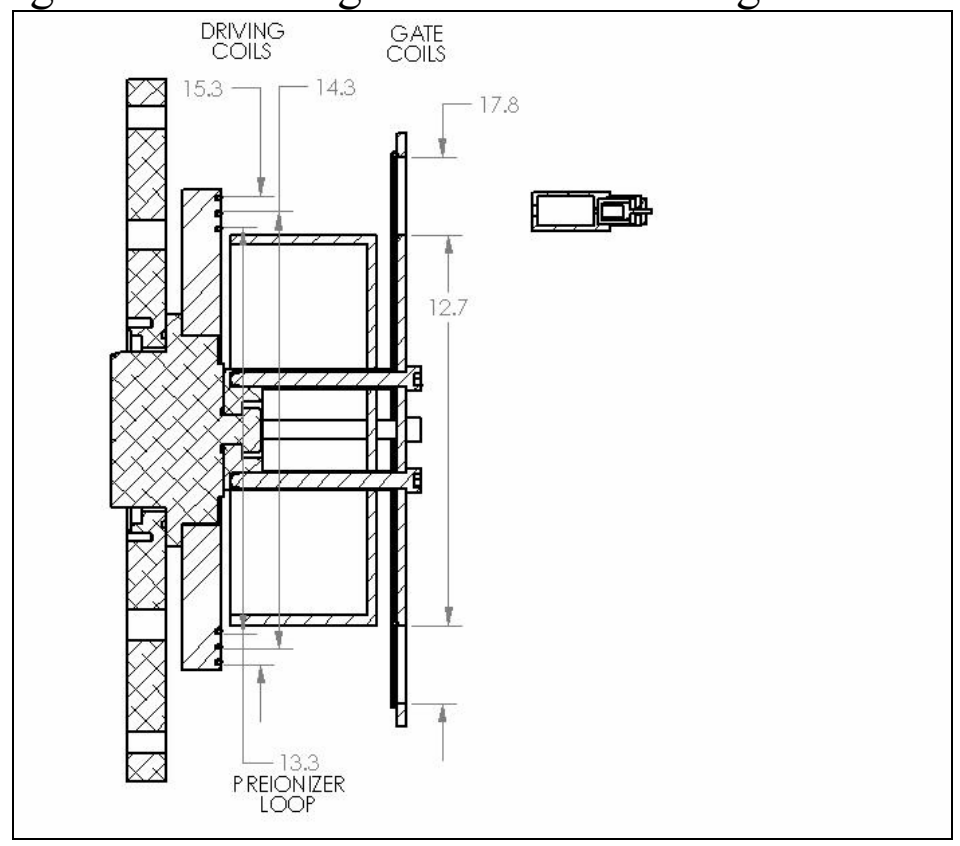


Picture 1: View of Source from Front of Chamber

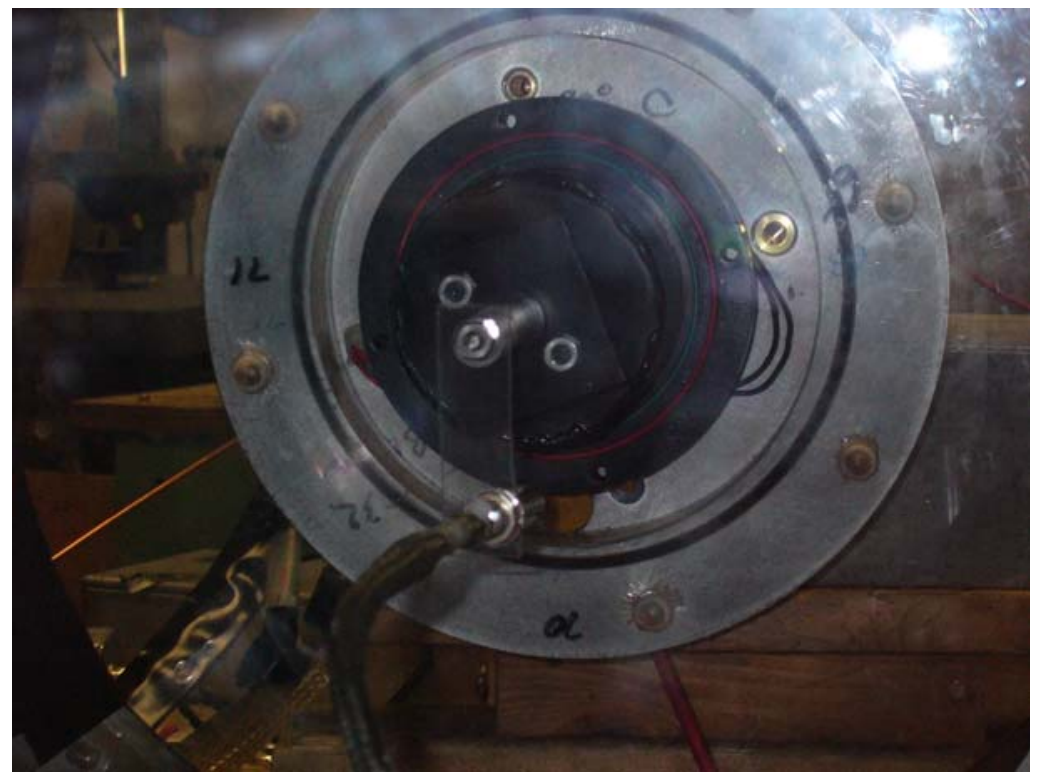

Picture 2: View of Chamber

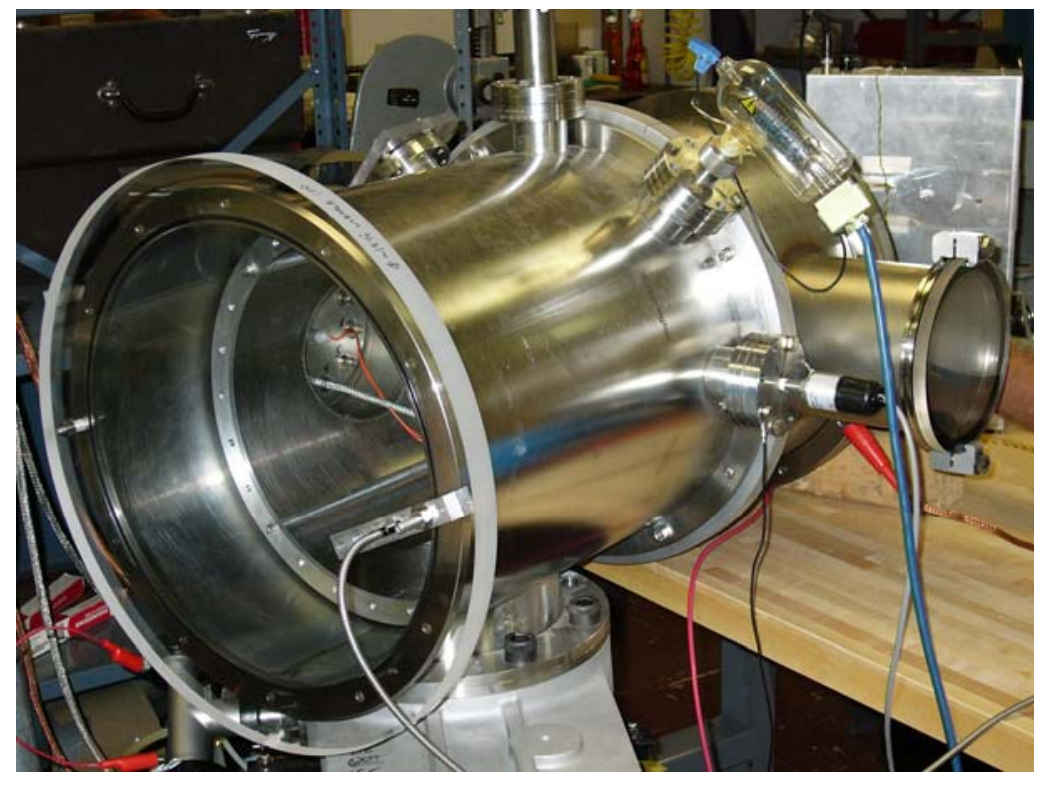


Figure 1: Effect of Power of Modulation on Ion Current

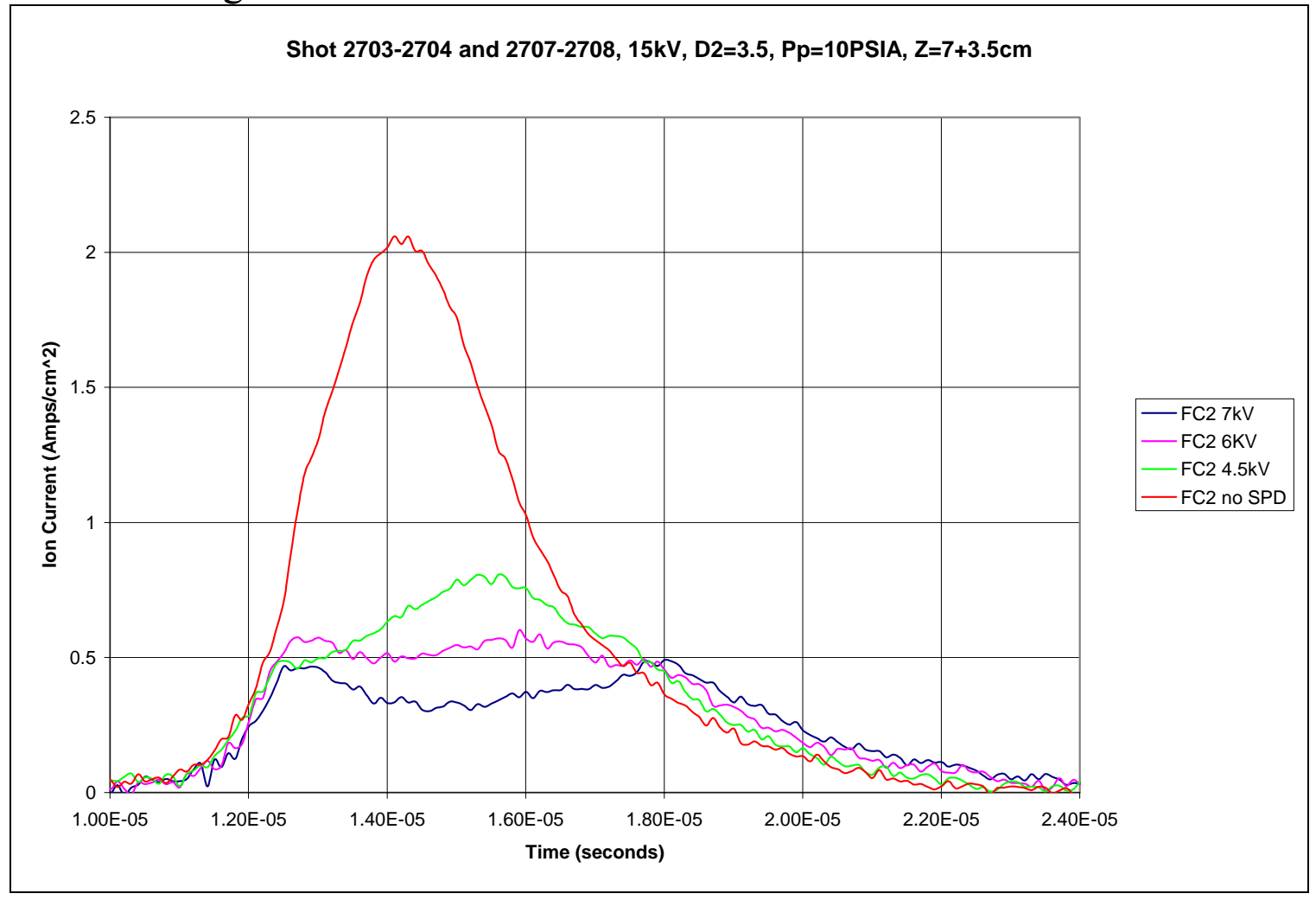

Figure 2: Effect of Time of Modulation on Ion Current

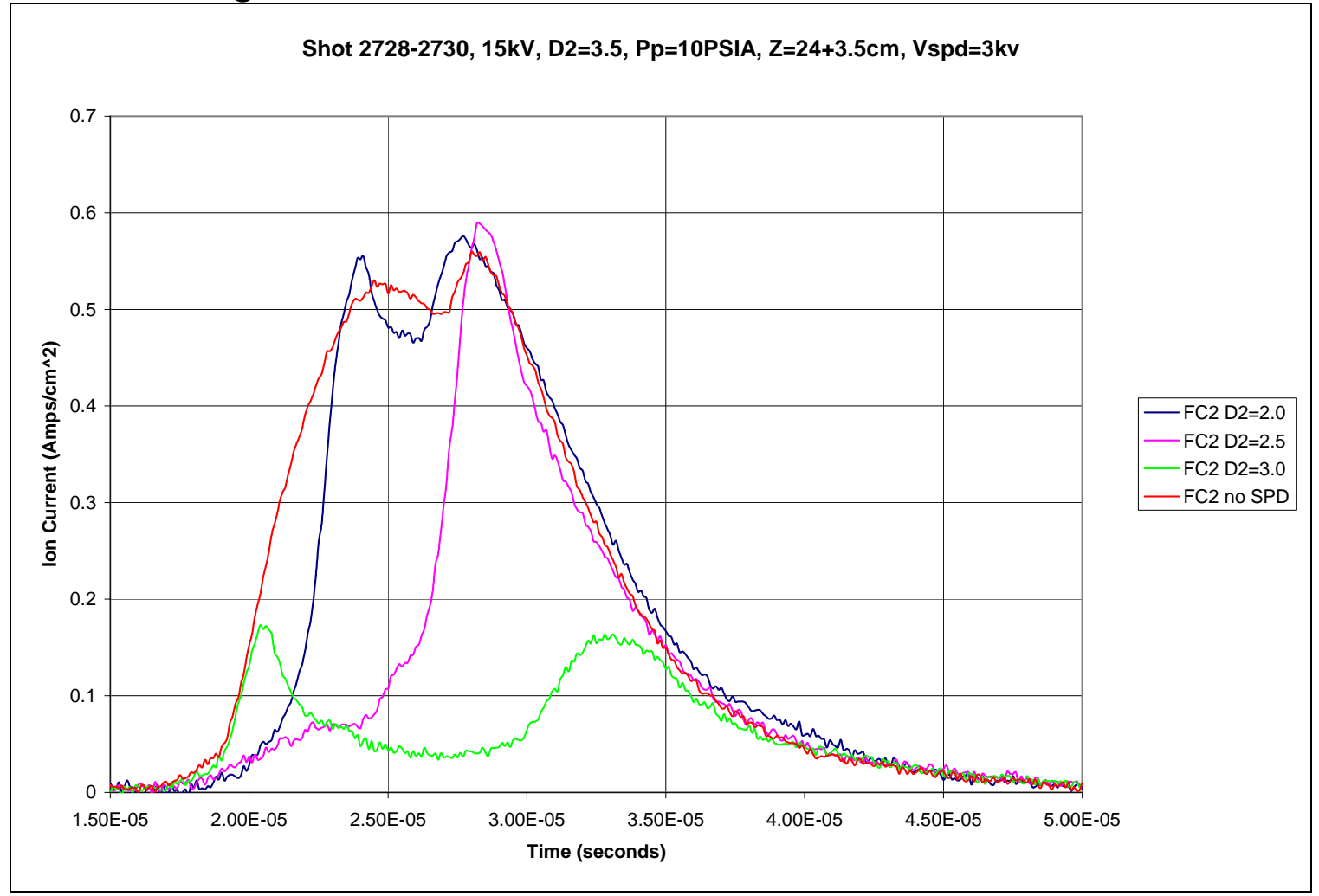


Figure 3: Normalized Peak Ion Current versus Faraday Cup Angle for Selected Radii at $22.3 \mathrm{~cm}$ from Source

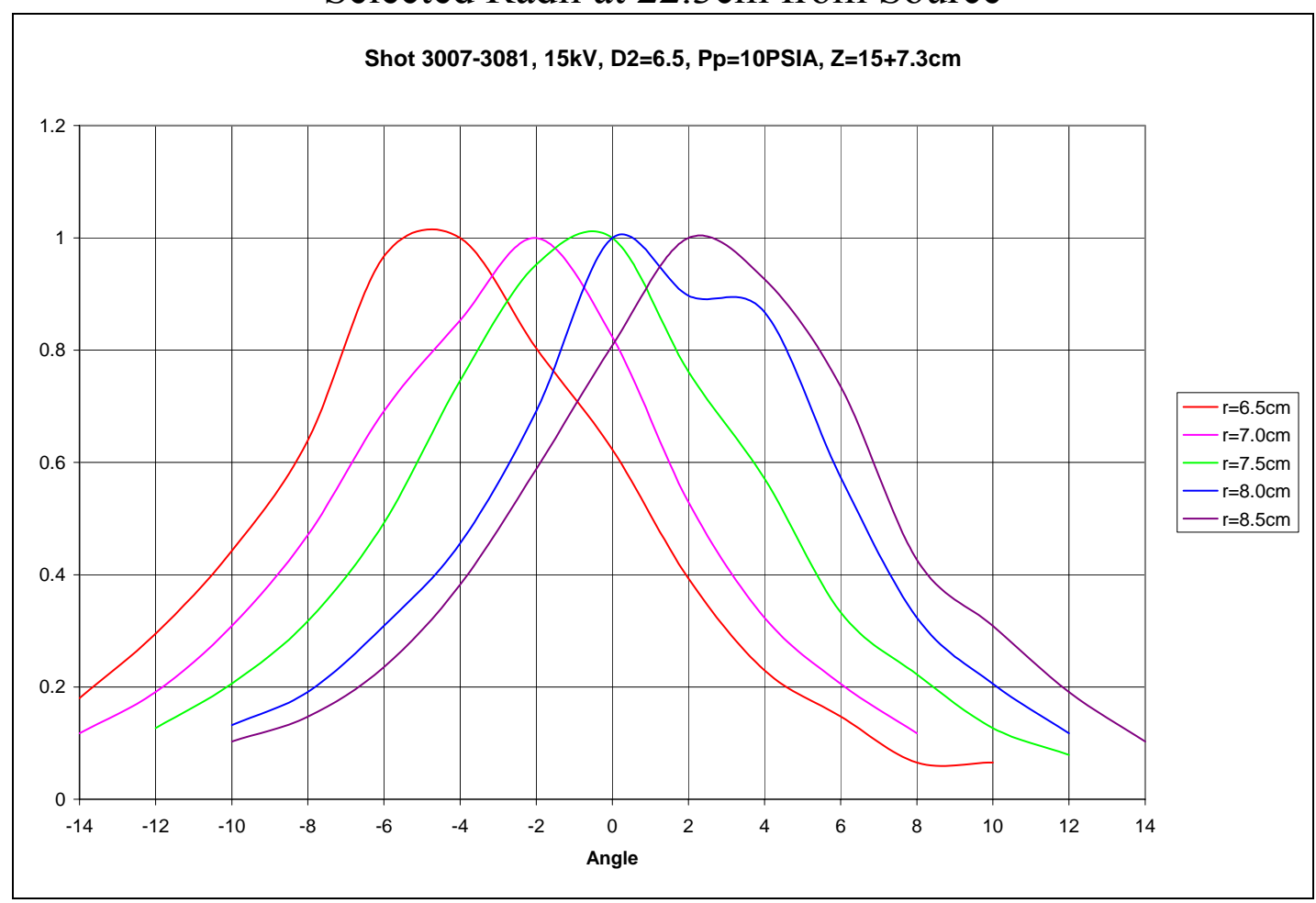

Figure 4: Normalized Peak Ion Current versus Faraday Cup Angle for Selected Radii at $32.3 \mathrm{~cm}$ from Source

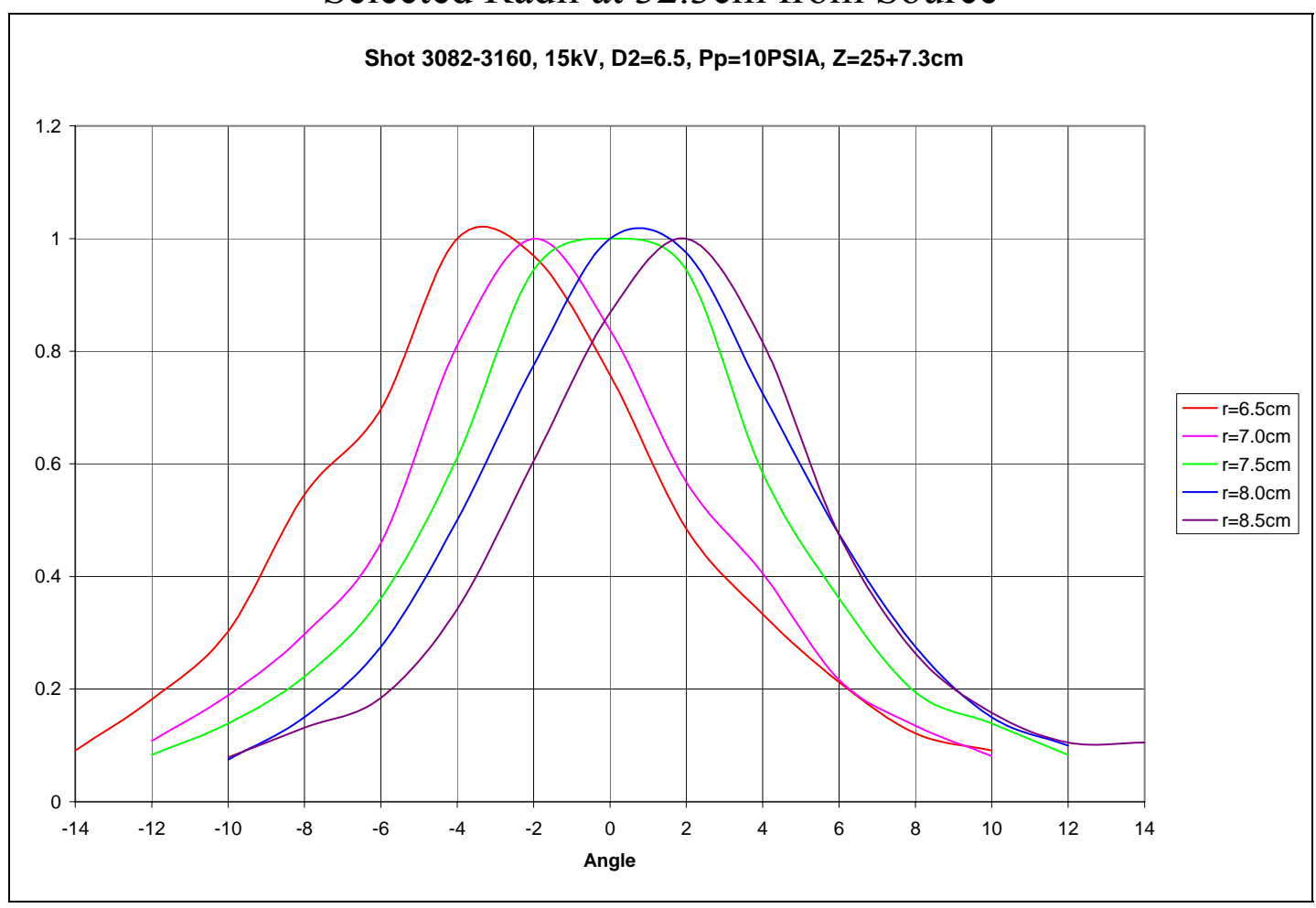


Figure 5: Peak Ion Current versus Faraday Cup Angle for Selected Radii at $22.3 \mathrm{~cm}$ from Source

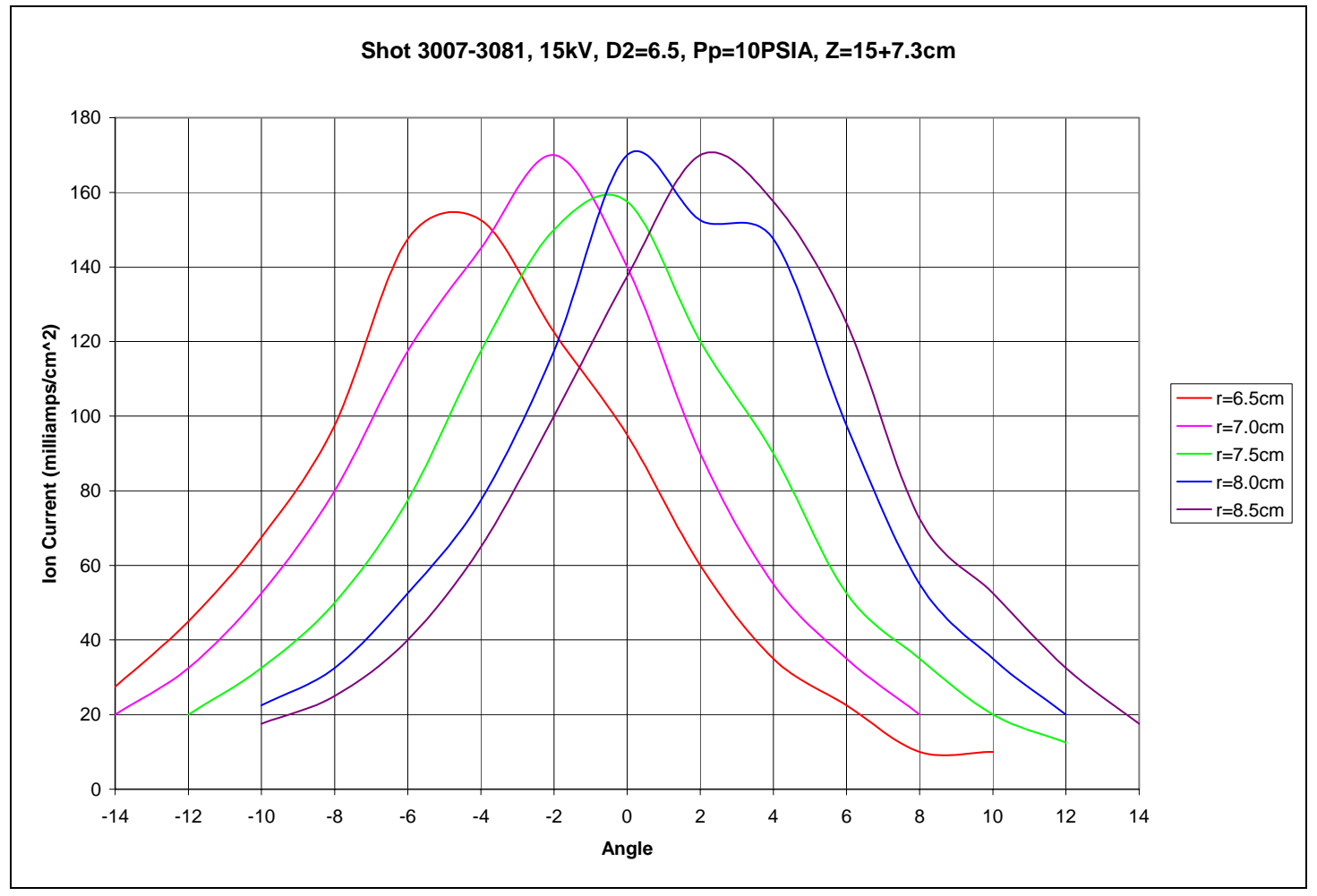

Figure 6: Peak Ion Current versus Faraday Cup Angle for Selected Radii at $32.3 \mathrm{~cm}$ from Source

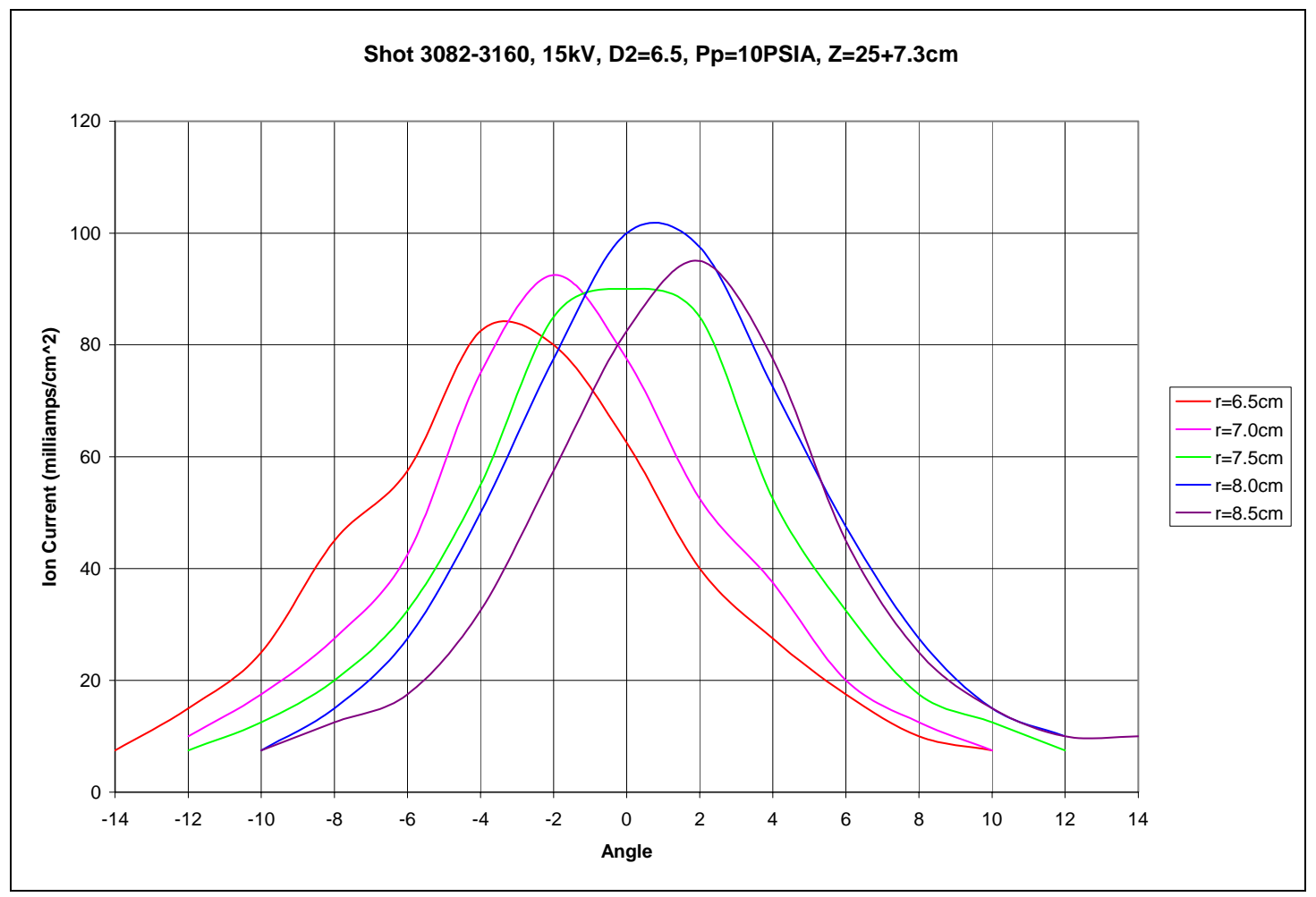


Table 1: Single Standard Deviation Divergence Calculated Using Specified Gaussian Width

\begin{tabular}{|c|c|c|}
\hline Width & $\mathrm{Z}=22.3 \mathrm{~cm}$ & $\mathrm{Z}=33.3 \mathrm{~cm}$ \\
\hline$+/-2^{\mathrm{o}}$ & $2.8^{\mathrm{o}}$ & $-\mathrm{NA}-$ \\
\hline$+/-4^{\mathrm{o}}$ & $3.3^{\mathrm{o}}$ & $2.2^{\mathrm{o}}$ \\
\hline$+/-6^{\mathrm{o}}$ & $3.7^{\mathrm{o}}$ & $3.1^{\mathrm{o}}$ \\
\hline$+/-8^{\mathrm{o}}$ & $4.1^{\mathrm{o}}$ & $3.7^{\mathrm{o}}$ \\
\hline$+/-10^{\mathrm{o}}$ & $4.6^{\mathrm{o}}$ & $4.3^{\mathrm{o}}$ \\
\hline
\end{tabular}

Table 2: Observed Angle of Peak Ion Current versus Radius

\begin{tabular}{|c|c|c|}
\hline Radius & $\mathrm{Z}=22.3 \mathrm{~cm}$ & $\mathrm{Z}=33.3 \mathrm{~cm}$ \\
\hline $6.0 \mathrm{~cm}$ & $-\mathrm{NA}-$ & $-4.0^{\circ}$ \\
\hline $6.5 \mathrm{~cm}$ & $-4.8^{\circ}$ & $-3.5^{\circ}$ \\
\hline $7.0 \mathrm{~cm}$ & $-2.0^{\circ}$ & $-2.0^{\circ}$ \\
\hline $7.5 \mathrm{~cm}$ & $-0.6^{\circ}$ & $0.0^{\circ}$ \\
\hline $8.0 \mathrm{~cm}$ & $0.2^{\circ}$ & $0.8^{\circ}$ \\
\hline $8.5 \mathrm{~cm}$ & $2.2^{\circ}$ & $1.9^{\circ}$ \\
\hline $9.0 \mathrm{~cm}$ & $5.0^{\circ}$ & $-\mathrm{NA}-$ \\
\hline $9.5 \mathrm{~cm}$ & $-\mathrm{NA}-$ & $2.2^{\circ}$ \\
\hline
\end{tabular}

Table 3: Single Standard Deviation Divergence Calculated Using Specified Gaussian Width (modulated versus un-modulated)

\begin{tabular}{|c|c|c|}
\hline Width & $Z=22.3 \mathrm{~cm}$ & $\begin{array}{c}\mathrm{Z}=22.3 \mathrm{~cm} \\
\text { (modulated) }\end{array}$ \\
\hline$+/-2^{\circ}$ & $2.8^{\circ}$ & $4.6^{\circ}$ \\
\hline$+/-4^{\circ}$ & $3.3^{\circ}$ & $3.9^{\circ}$ \\
\hline$+/-6^{\circ}$ & $3.7^{\circ}$ & $4.2^{\circ}$ \\
\hline$+/-8^{\circ}$ & $4.1^{\circ}$ & $4.3^{\circ}$ \\
\hline$+/-10^{\circ}$ & $4.6^{\circ}$ & $4.85^{\circ}$ \\
\hline
\end{tabular}


Table 4: Observed Angle of Peak Ion Current versus Radius (modulated versus un-modulated)

\begin{tabular}{|c|c|c|}
\hline Radius & $\mathrm{Z}=22.3 \mathrm{~cm}$ & $\begin{array}{c}\mathrm{Z}=22.3 \mathrm{~cm} \\
\text { (modulated) }\end{array}$ \\
\hline $6.0 \mathrm{~cm}$ & $-\mathrm{NA}-$ & $-6.1^{\mathrm{o}}$ \\
\hline $6.5 \mathrm{~cm}$ & $-4.8^{\circ}$ & $-4.5^{\mathrm{o}}$ \\
\hline $7.0 \mathrm{~cm}$ & $-2.0^{\circ}$ & $-2.2^{\circ}$ \\
\hline $7.5 \mathrm{~cm}$ & $-0.6^{\circ}$ & $-1.6^{\circ}$ \\
\hline $8.0 \mathrm{~cm}$ & $0.2^{\circ}$ & $0.6^{\circ}$ \\
\hline $8.5 \mathrm{~cm}$ & $2.2^{\circ}$ & $3.0^{\circ}$ \\
\hline $9.0 \mathrm{~cm}$ & $5.0^{\circ}$ & $-\mathrm{NA}-$ \\
\hline
\end{tabular}


Figure 7: Normalized Peak Ion Current versus Faraday Cup Angle for Selected Radii at $22.3 \mathrm{~cm}$ from Modulated Source

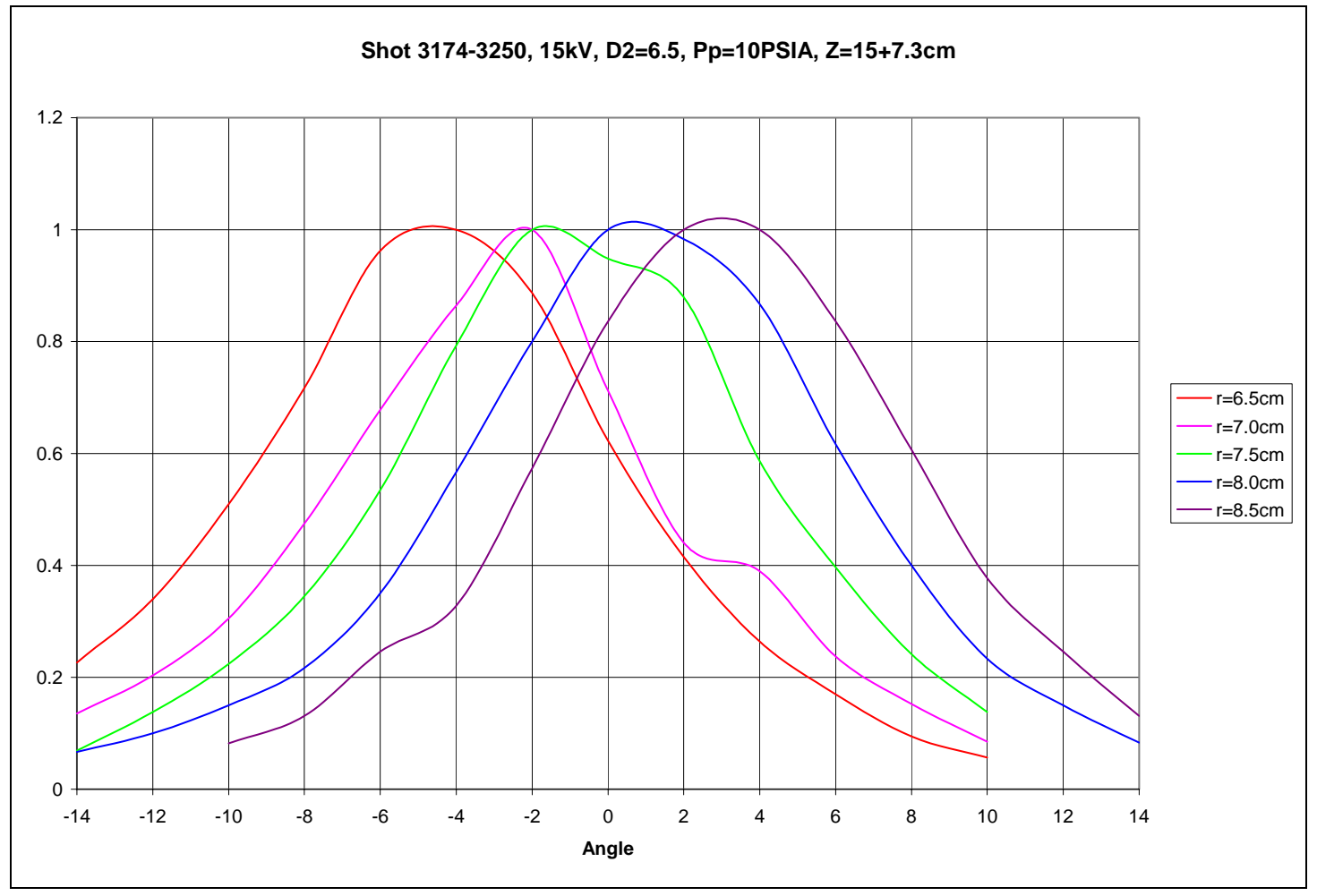

Figure 8: Peak Ion Current versus Faraday Cup Angle for Selected Radii at $22.3 \mathrm{~cm}$ from Modulated Source

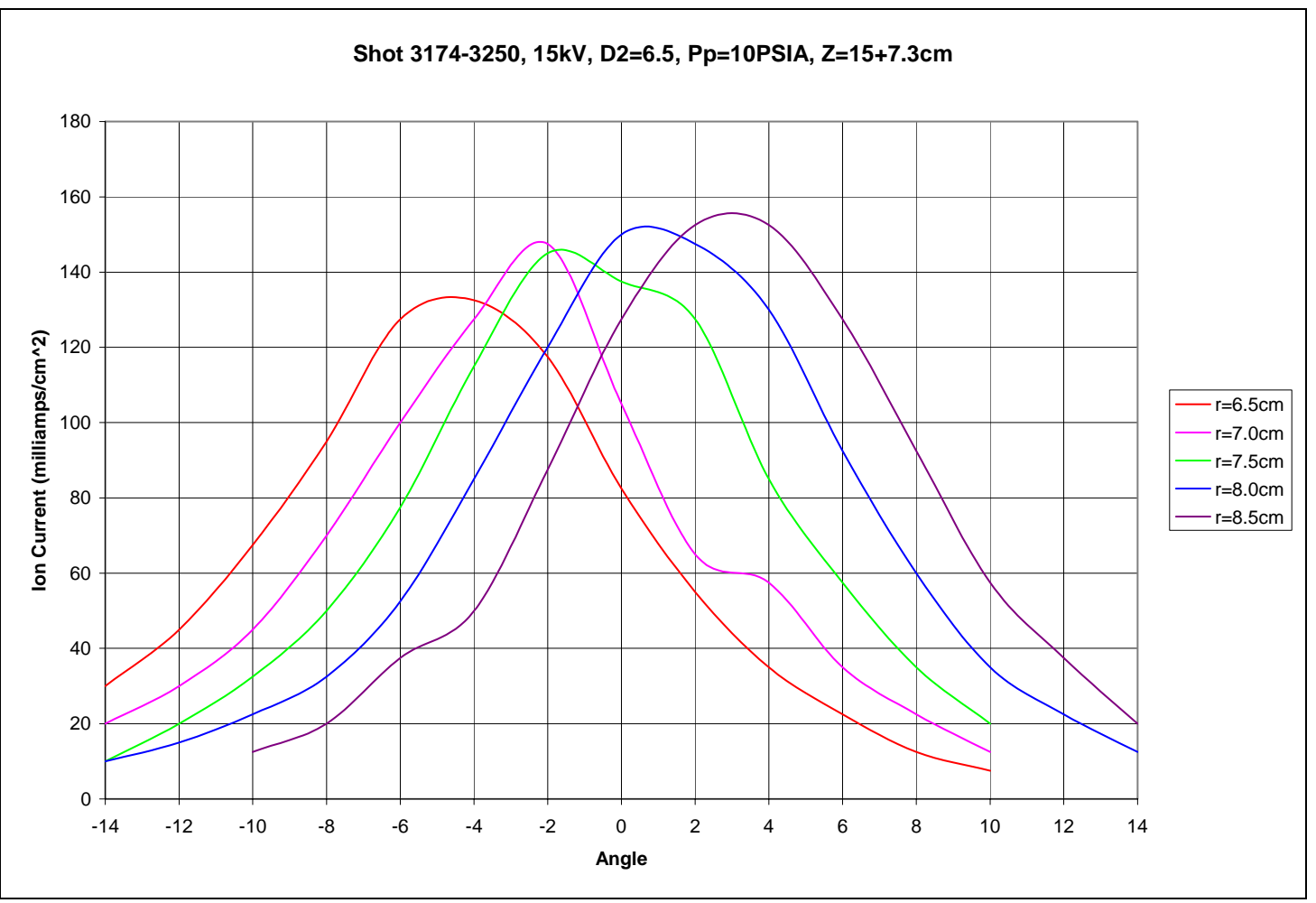


Figure 9: Ion Current versus Time at Different Distances from Source

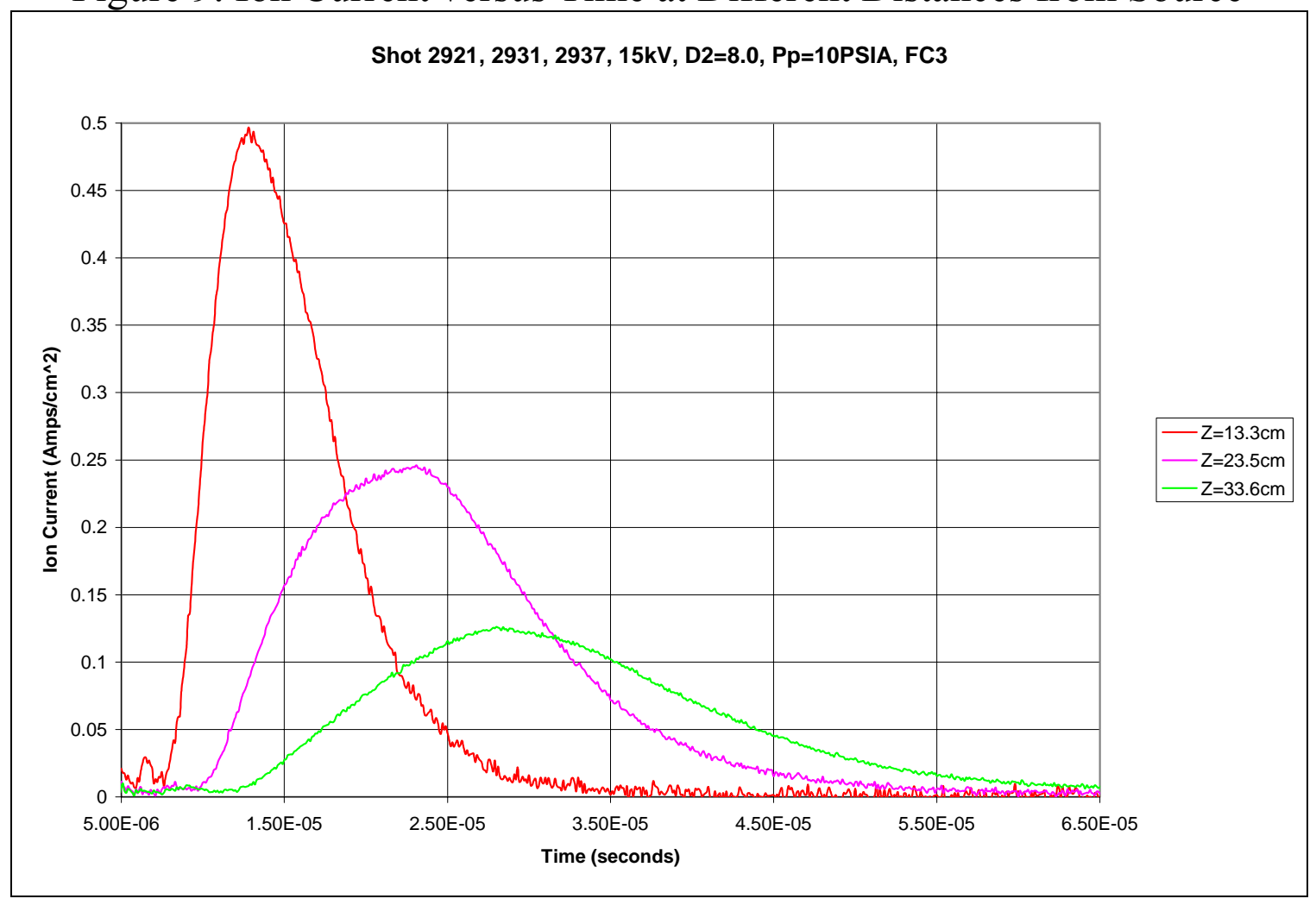

Table 5: Measured Time to Specified Ion Current Level

\begin{tabular}{|c|c|c|c|}
\hline $\begin{array}{c}\text { Percent on } \\
\text { Rise }\end{array}$ & $Z=13.3 \mathrm{~cm}$ & $Z=23.5 \mathrm{~cm}$ & $Z=33.6 \mathrm{~cm}$ \\
\hline $10 \%$ & $8.4 \mu \mathrm{s}$ & $10.9 \mu \mathrm{s}$ & $13.3 \mu \mathrm{s}$ \\
\hline $50 \%$ & $9.9 \mu \mathrm{s}$ & $13.8 \mu \mathrm{s}$ & $18.5 \mu \mathrm{s}$ \\
\hline $90 \%$ & $11.6 \mu \mathrm{s}$ & $18.7 \mu \mathrm{s}$ & $24.9 \mu \mathrm{s}$ \\
\hline
\end{tabular}

Table 6: Calculated Velocity for Specified Ion Current Level

\begin{tabular}{|c|c|c|c|}
\hline $\begin{array}{c}\text { Percent on } \\
\text { Rise }\end{array}$ & $\begin{array}{c}\mathrm{Z}=13.3 \mathrm{~cm} \\
\text { to } 23.5 \mathrm{~cm}\end{array}$ & $\begin{array}{c}\mathrm{Z}=23.5 \mathrm{~cm} \\
\text { to } 33.6 \mathrm{~cm}\end{array}$ & $\begin{array}{c}\mathrm{Z}=13.3 \mathrm{~cm} \\
\text { to } 33.6 \mathrm{~cm}\end{array}$ \\
\hline $10 \%$ & $4.08 \mathrm{~cm} / \mu \mathrm{s}$ & $4.21 \mathrm{~cm} / \mu \mathrm{s}$ & $4.14 \mathrm{~cm} / \mu \mathrm{s}$ \\
\hline $50 \%$ & $2.61 \mathrm{~cm} / \mu \mathrm{s}$ & $2.15 \mathrm{~cm} / \mu \mathrm{s}$ & $2.36 \mathrm{~cm} / \mu \mathrm{s}$ \\
\hline $90 \%$ & $1.43 \mathrm{~cm} / \mu \mathrm{s}$ & $1.63 \mathrm{~cm} / \mu \mathrm{s}$ & $1.53 \mathrm{~cm} / \mu \mathrm{s}$ \\
\hline
\end{tabular}

Table 7: Calculated Time of Origin of Ion Current Using Time of Flight Data

\begin{tabular}{|c|c|c|c|}
\hline & $Z=13.3 \mathrm{~cm}$ & $Z=23.5 \mathrm{~cm}$ & $Z=33.6 \mathrm{~cm}$ \\
\hline Origin Time & $4.57 \mu \mathrm{s}$ & $4.61 \mu \mathrm{s}$ & $4.57 \mu \mathrm{s}$ \\
\hline
\end{tabular}


Figure 10: Driving Current versus Time for Different Source Configurations

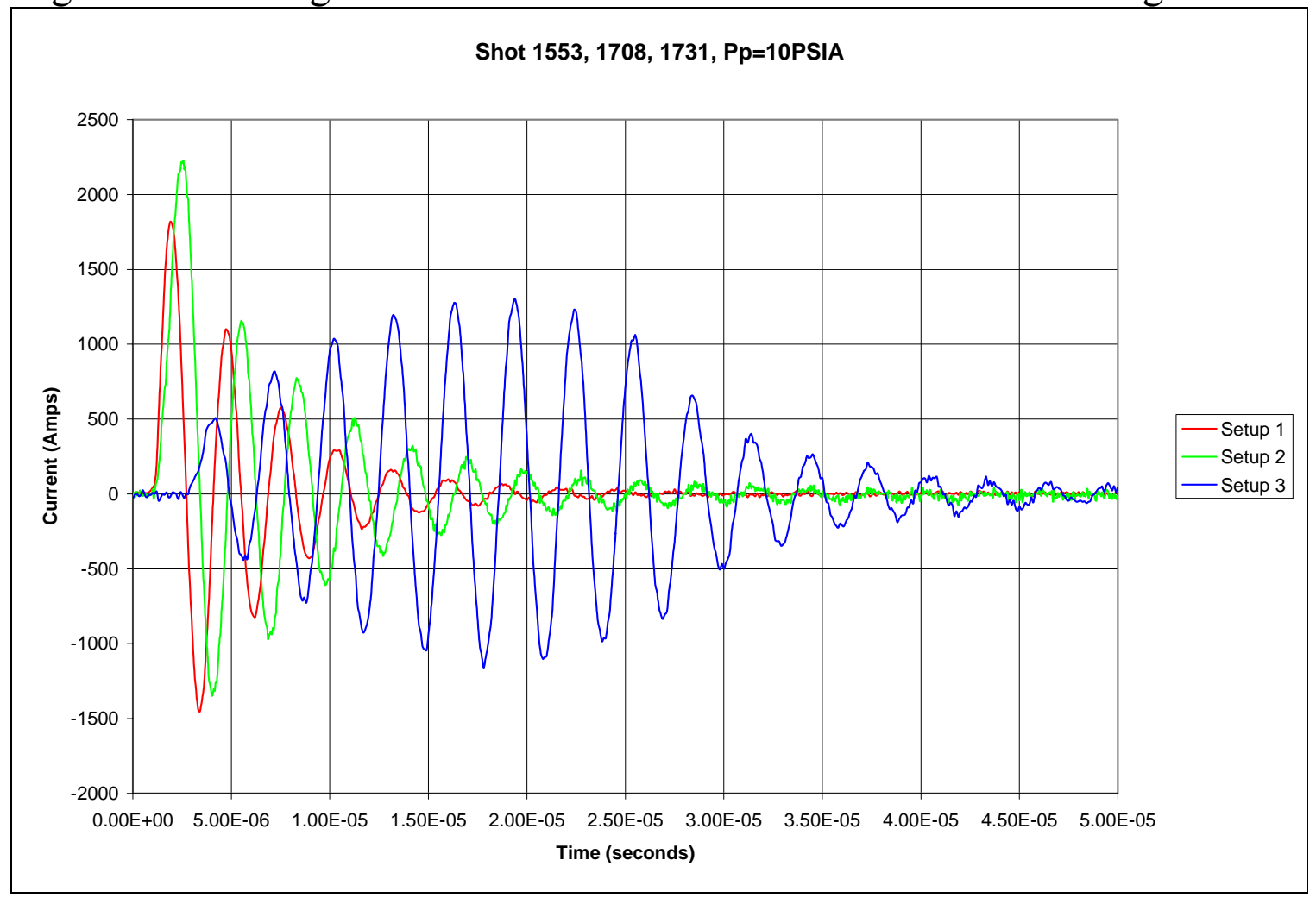

Figure 11: Ion Current versus Time for Different Source Configurations

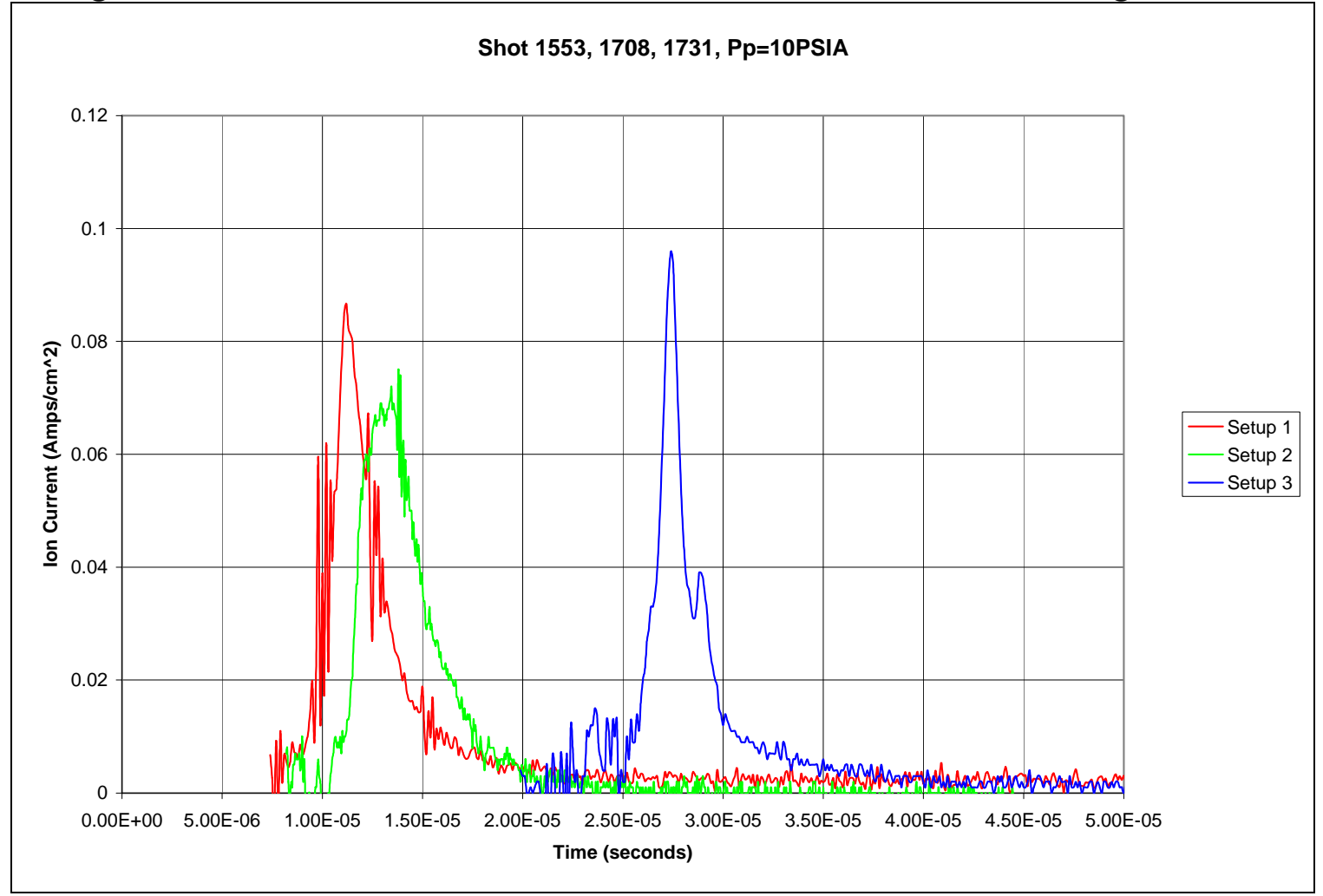


Figure 12: Timing of Front View Pictures Relative to Driving and Ion Currents

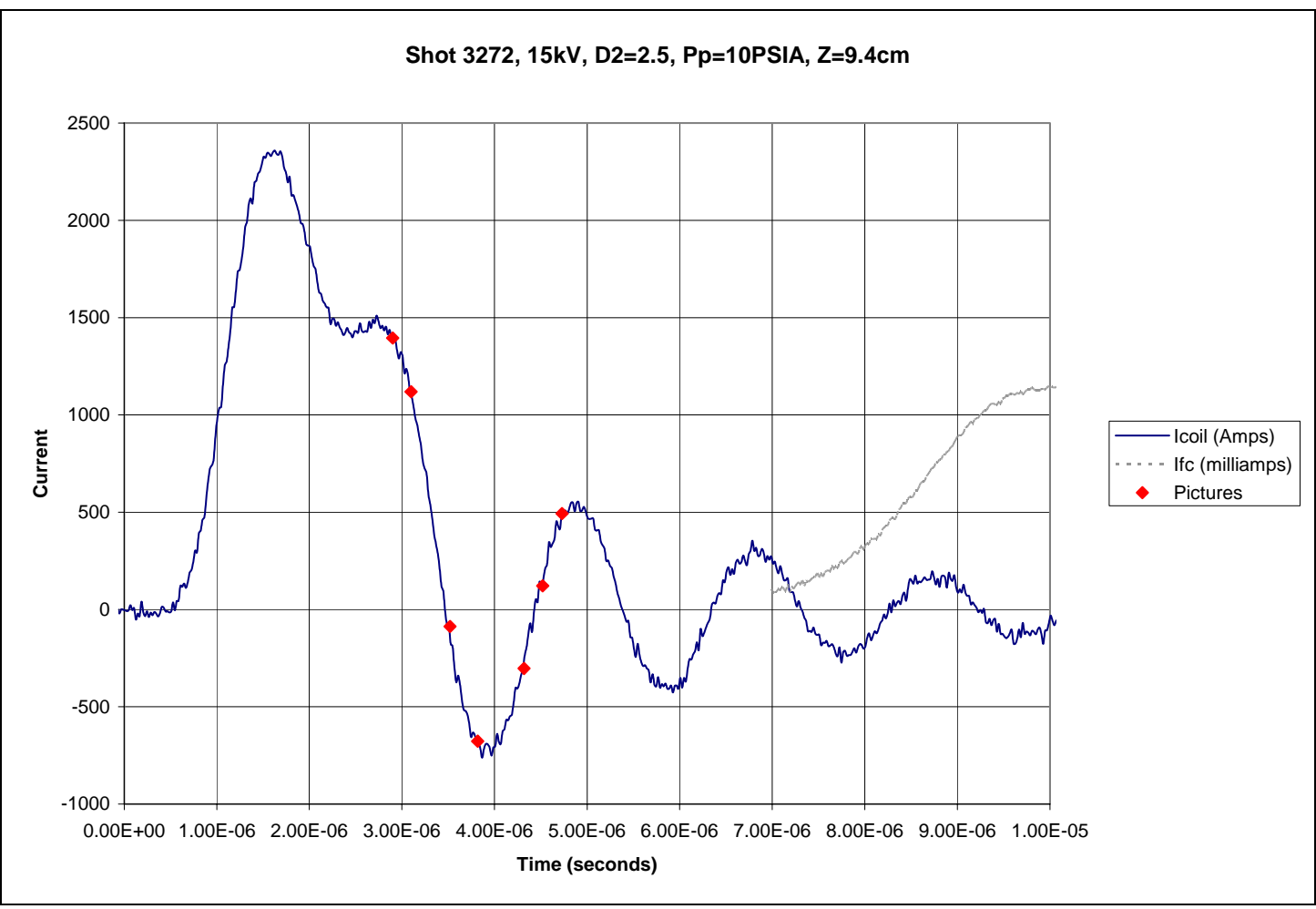

Figure 13: Timing of Side View Pictures Relative to Driving and Ion Currents

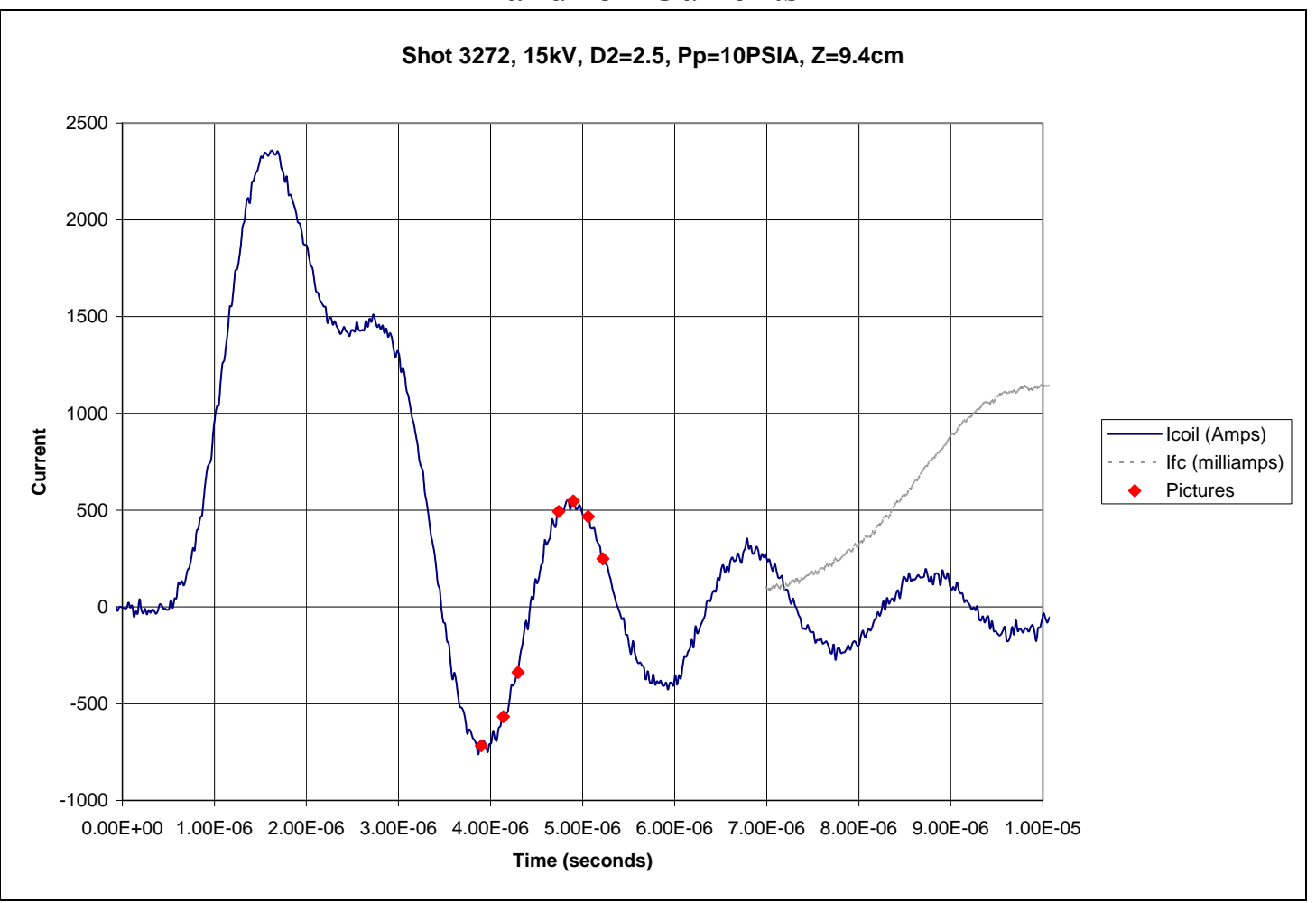




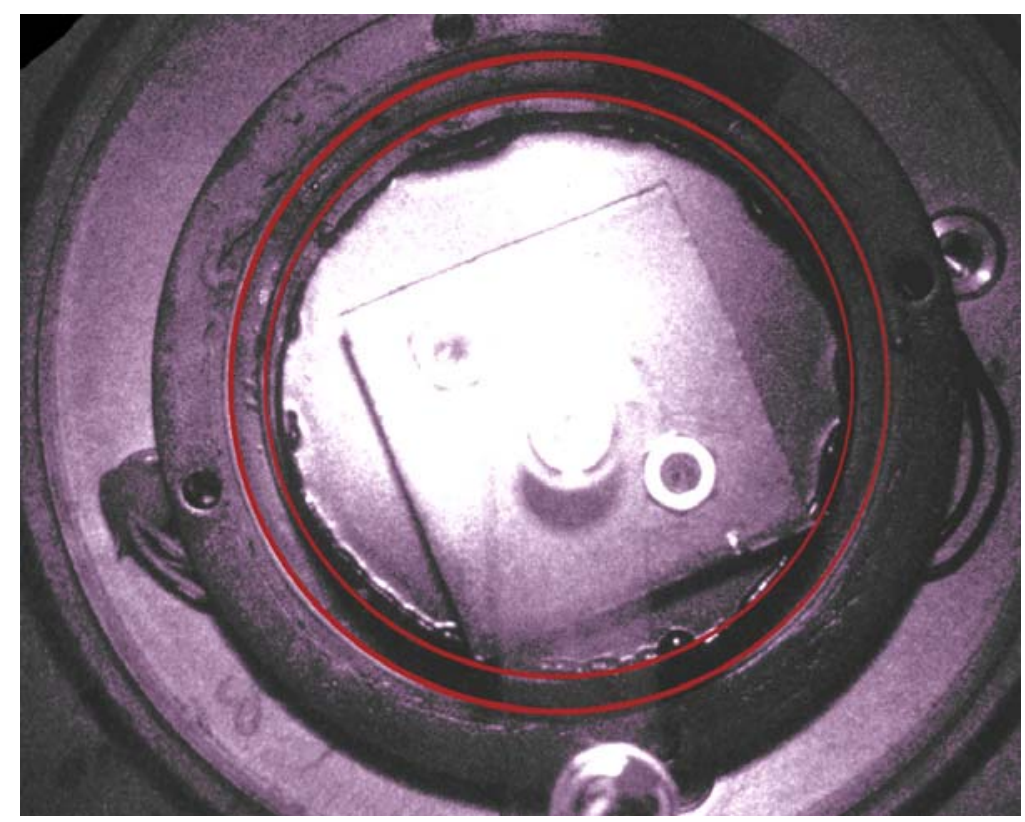

Picture 3: Front View Room Light

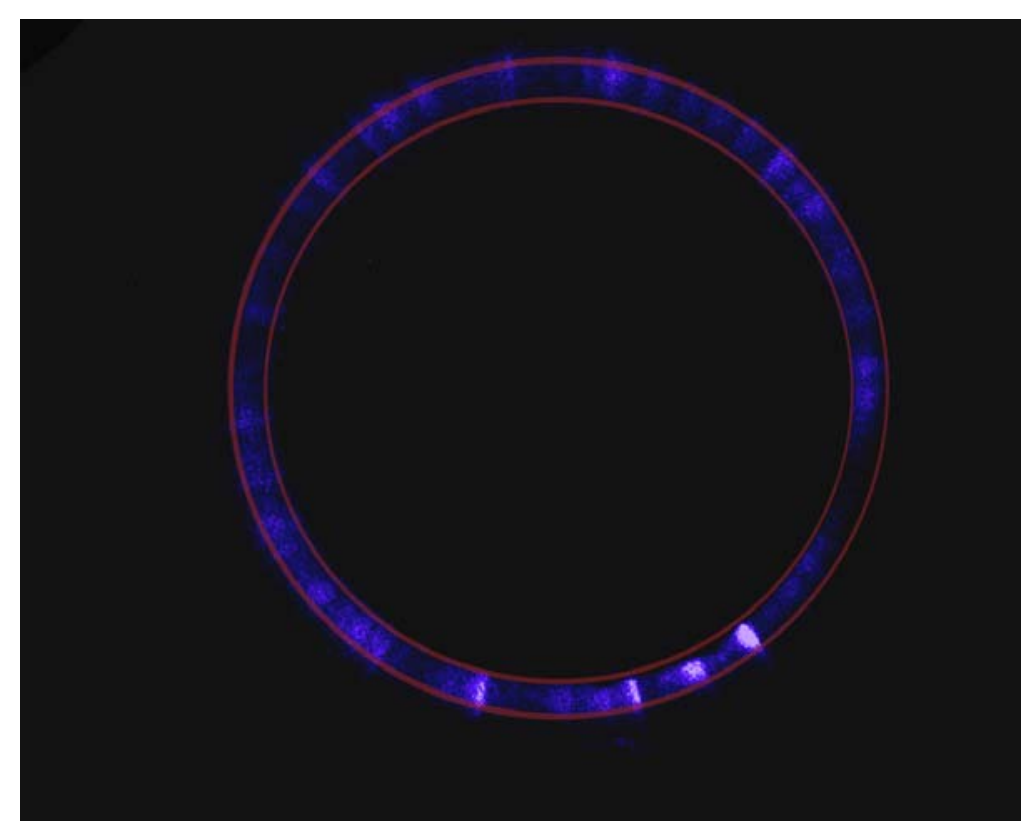

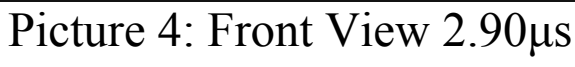


Applied Pulsed Power, Inc.

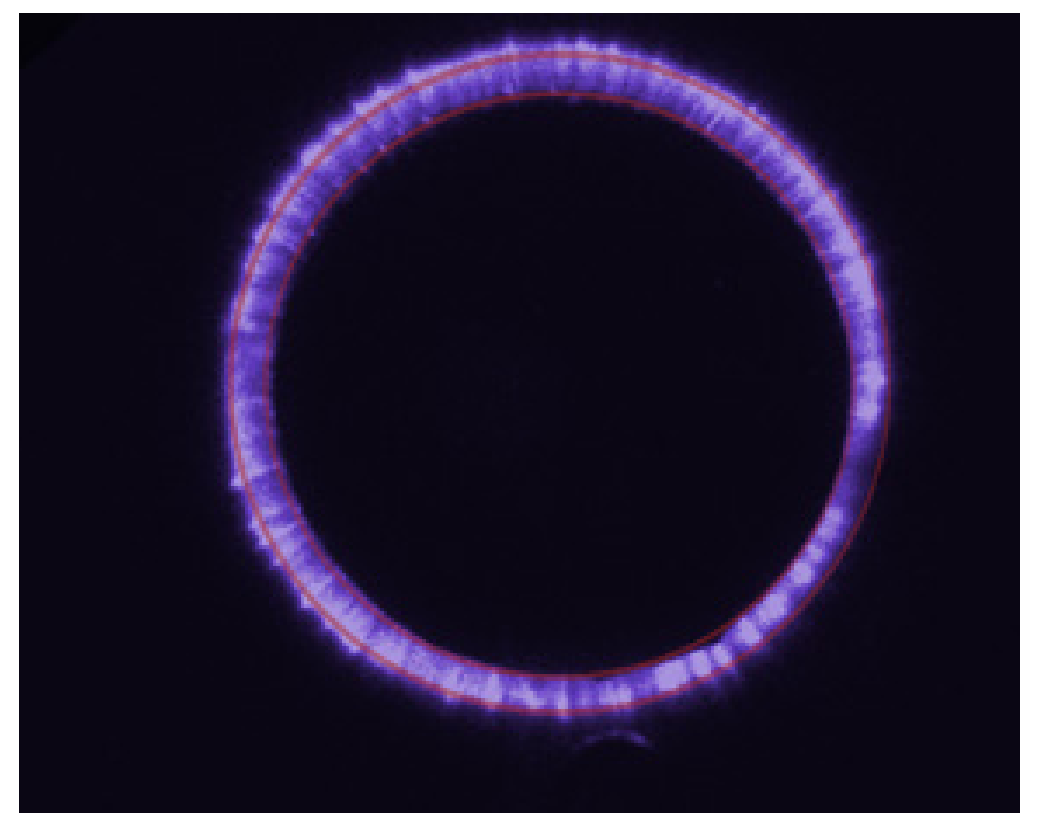

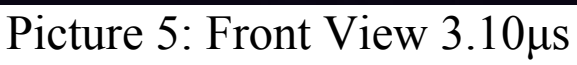

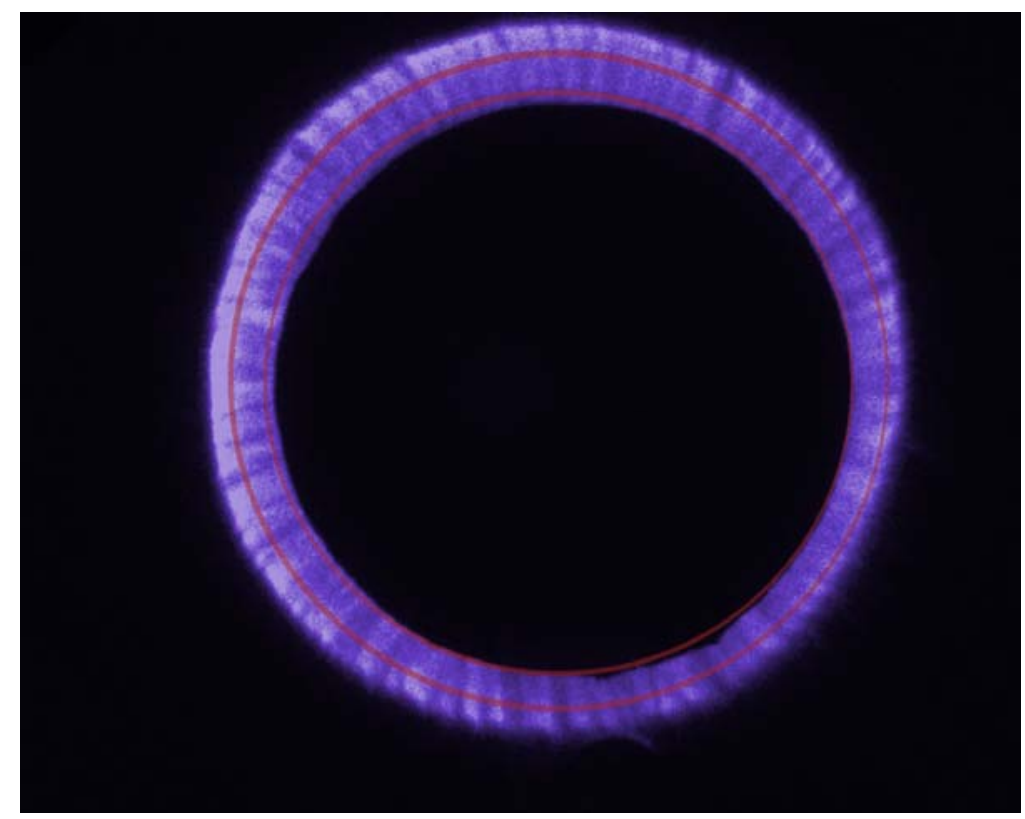

Picture 6: Front View 3.52 $\mu$ s 
Applied Pulsed Power, Inc.

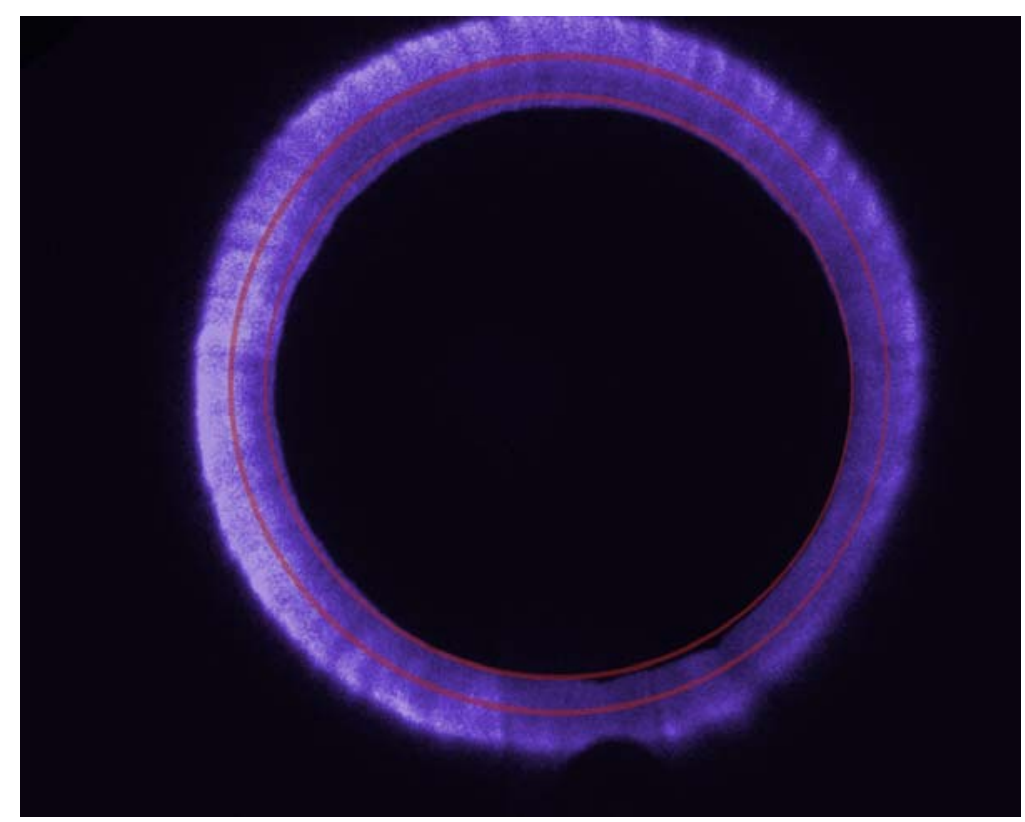

Picture 7: Front View 3.82 $\mu$ s

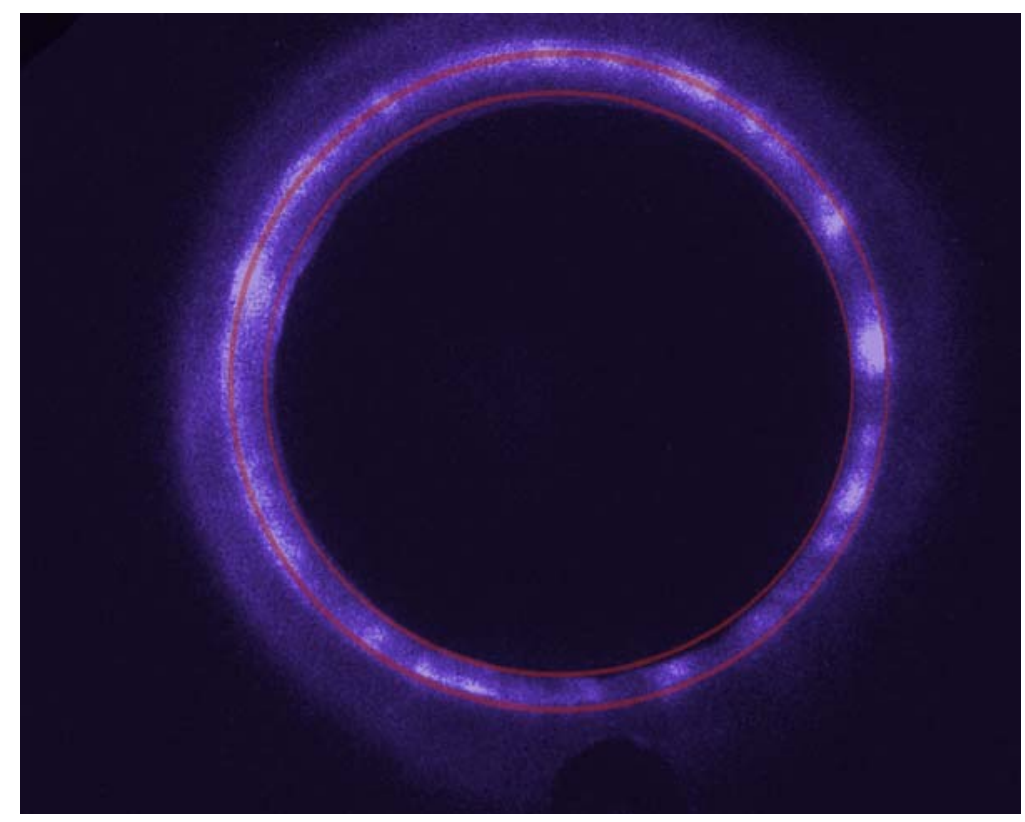

Picture 8: Front View 4.32 $\mu$ s 
Applied Pulsed Power, Inc.

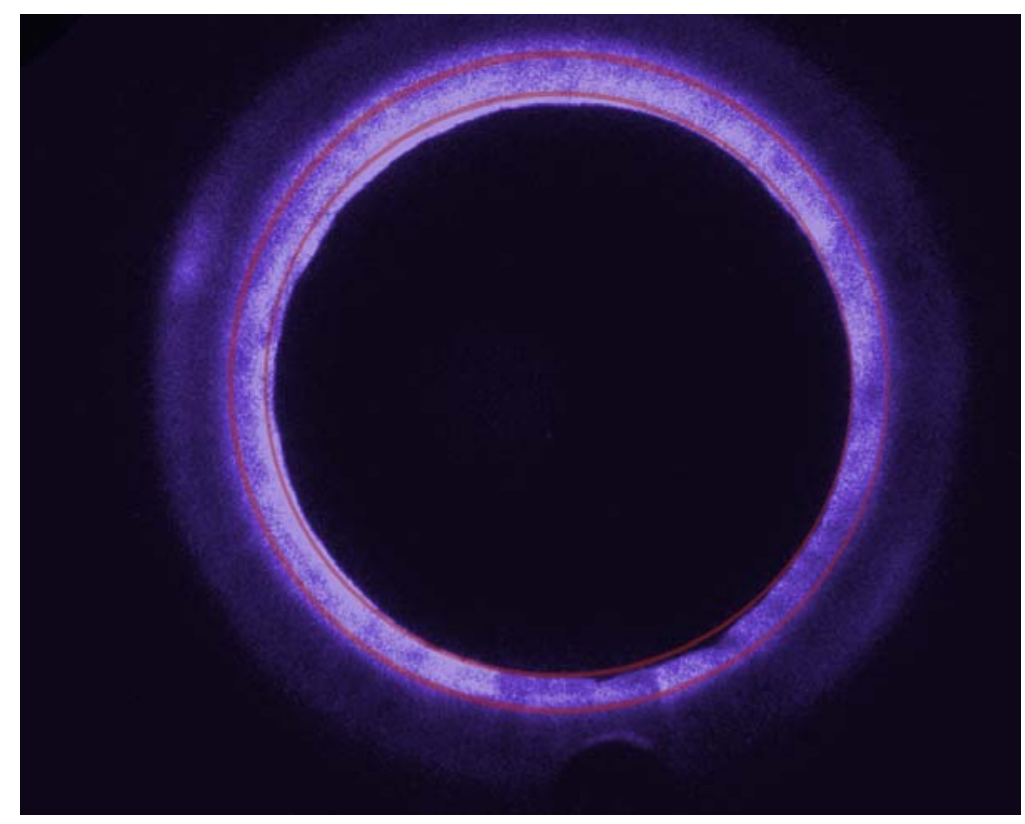

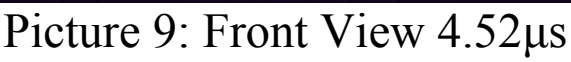

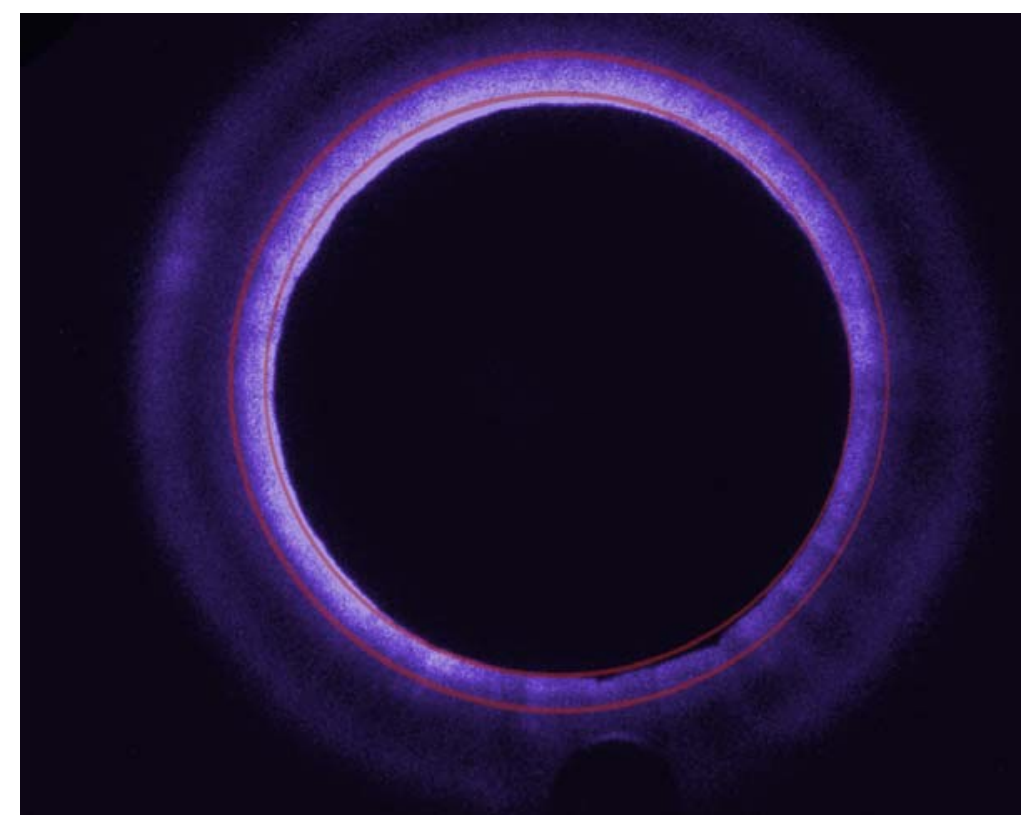

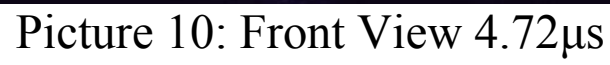


Applied Pulsed Power, Inc.

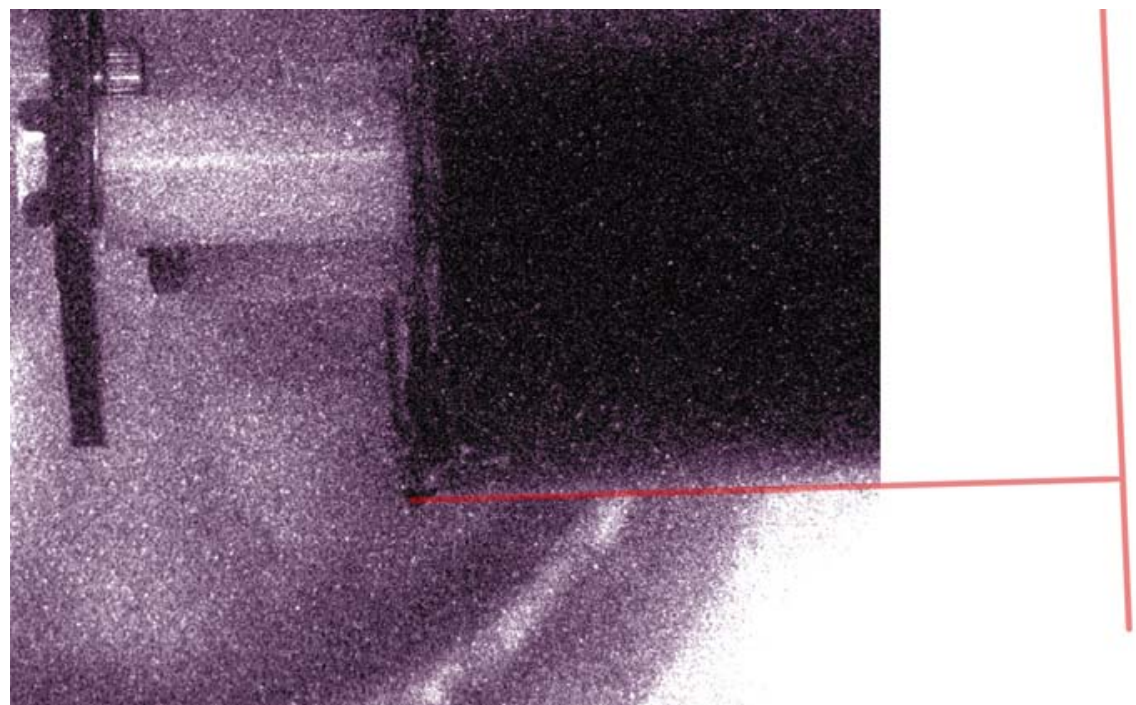

Picture 11: Side View Room Light

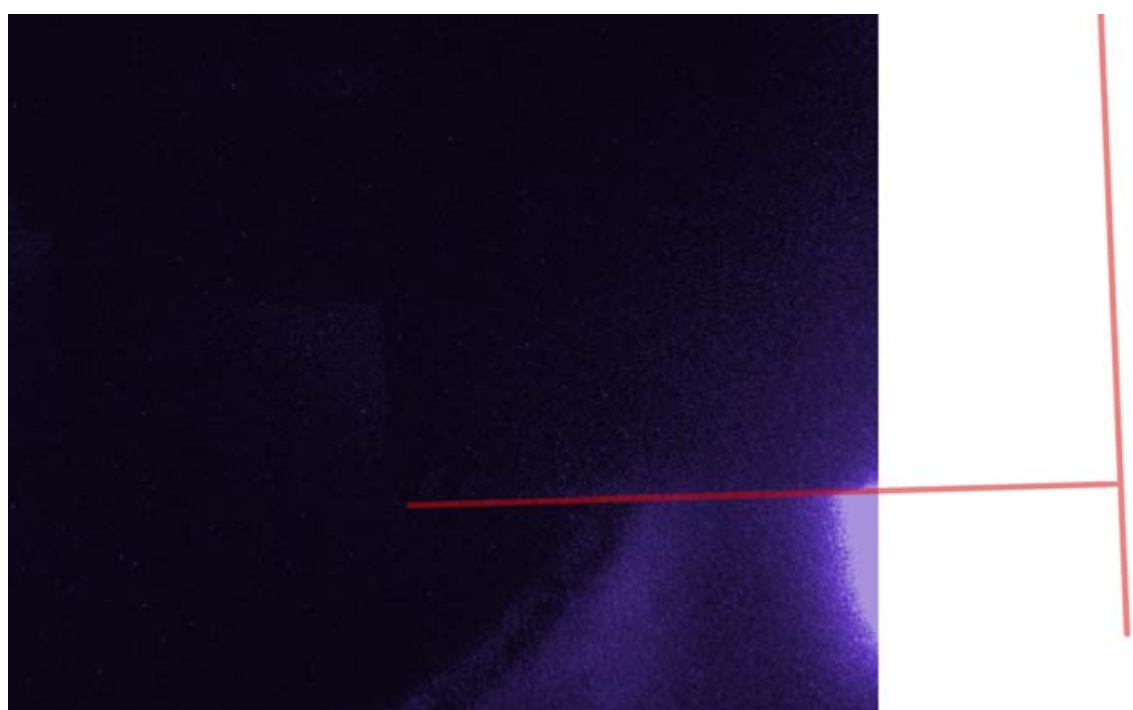

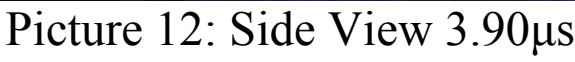

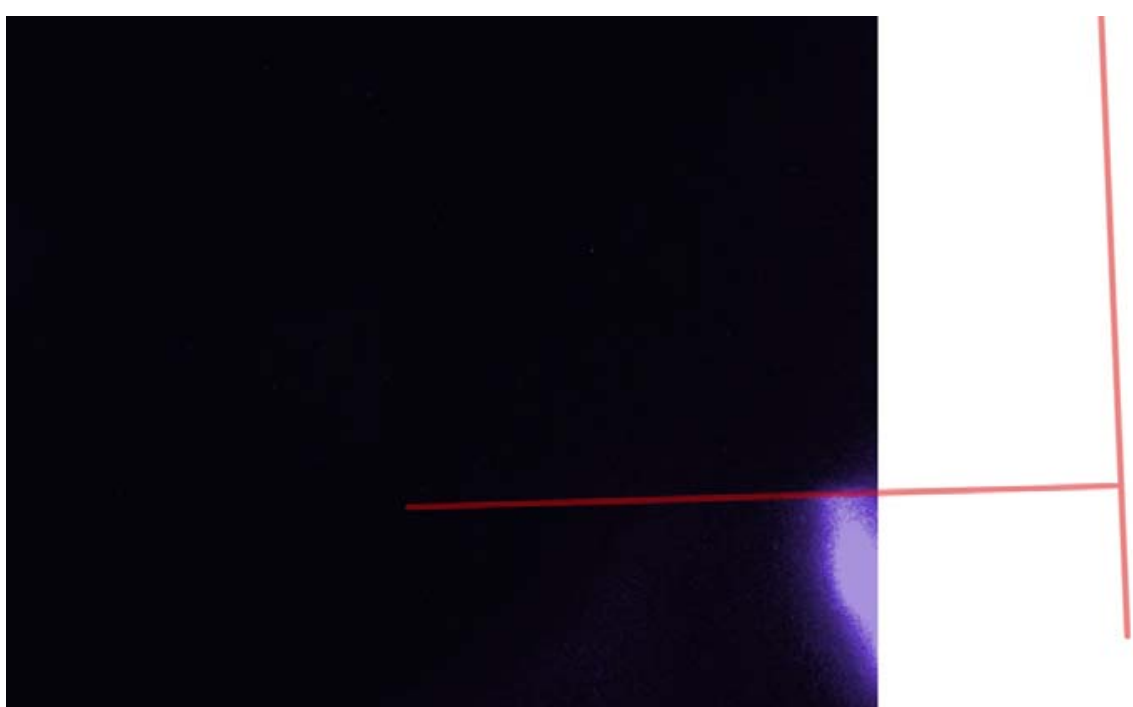

Picture 13: Side View 4.14 $\mu$ s 


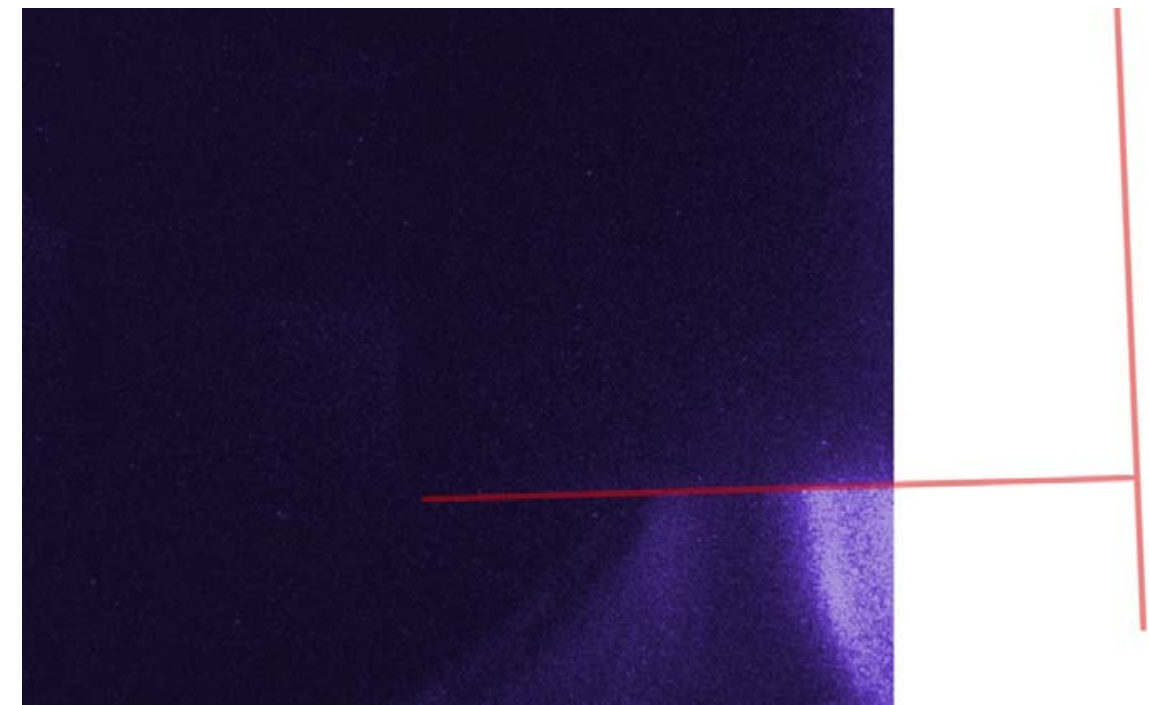

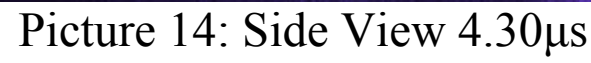

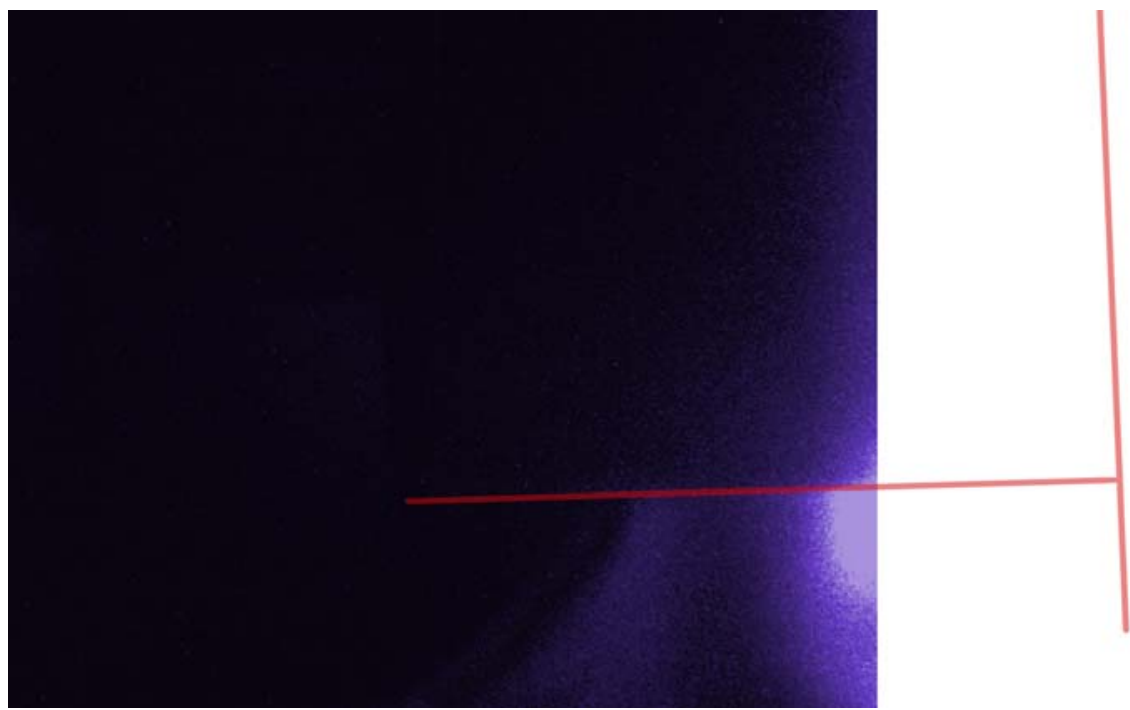

Picture 15: Side View 4.74 $\mu$ s

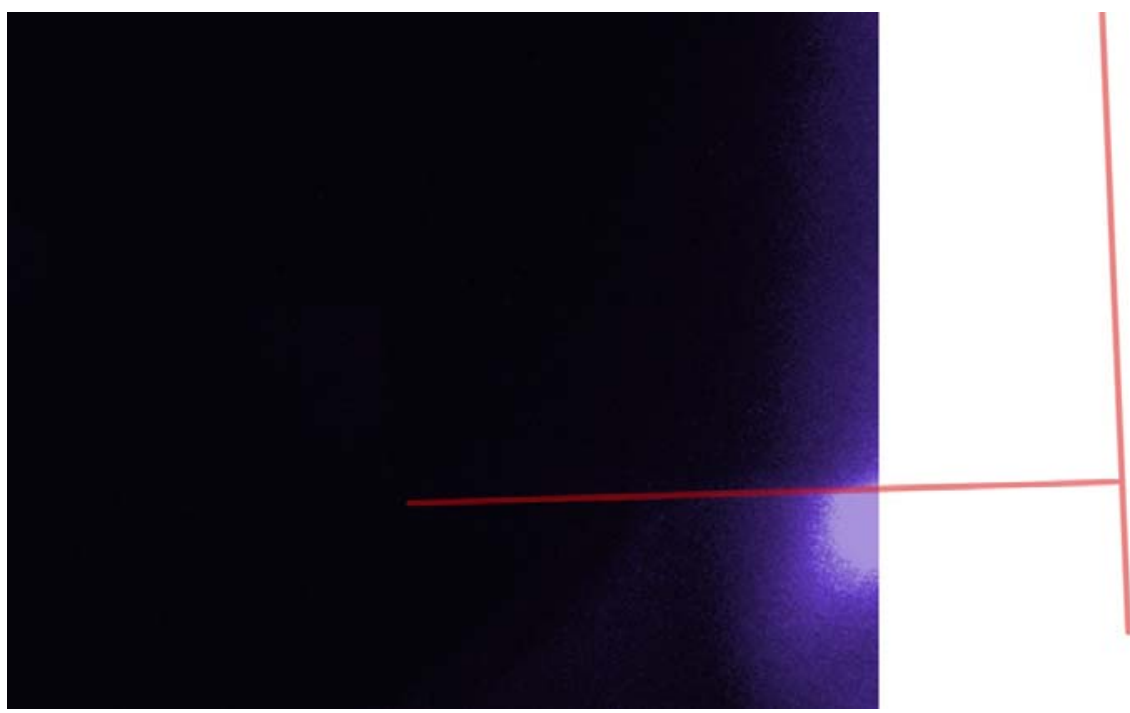


Applied Pulsed Power, Inc.

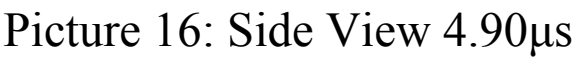

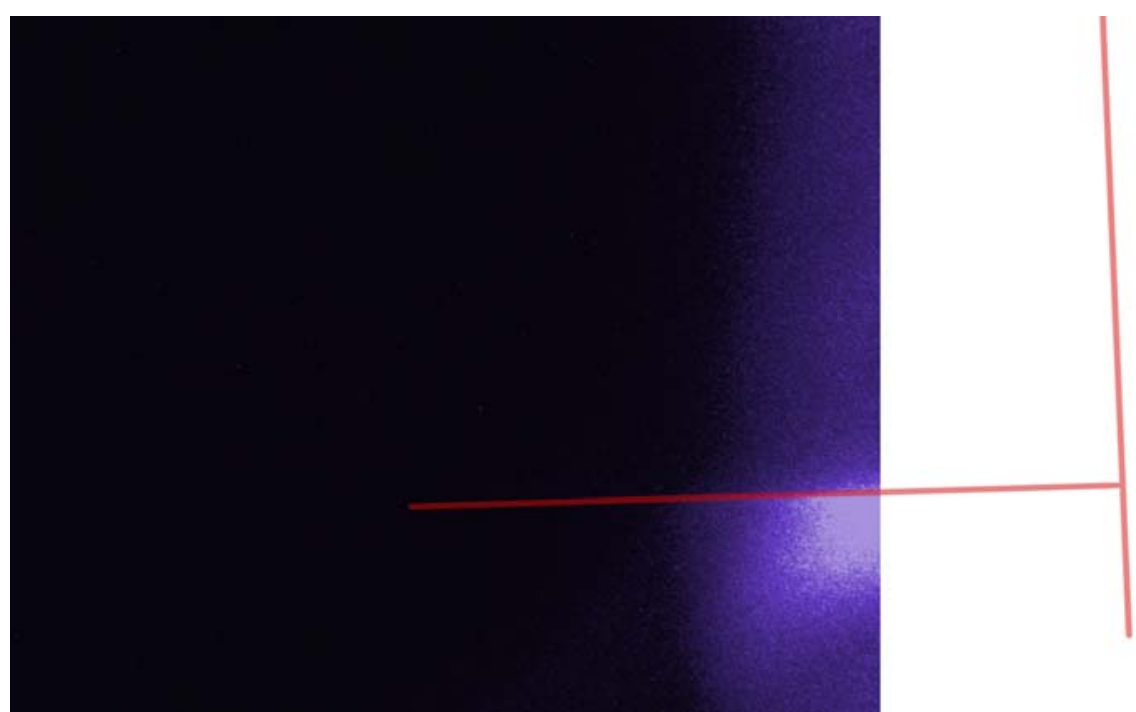

Picture 17: Side View 5.06 $\mu$ s

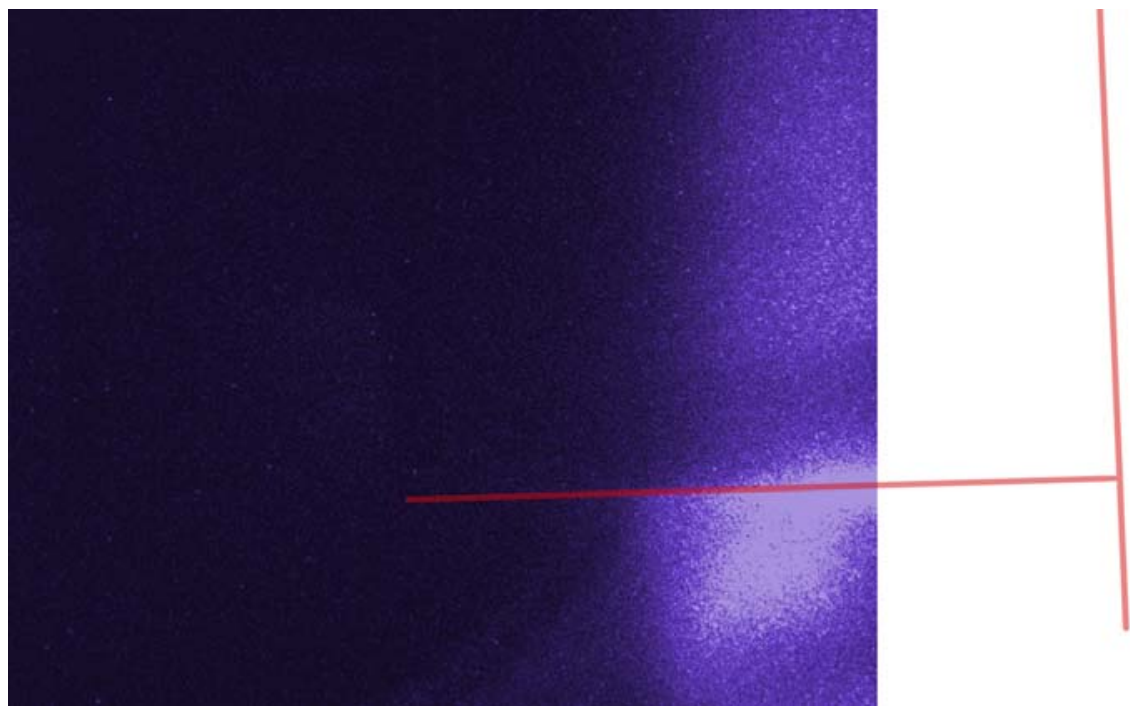

Picture 18: Side View 5.22 $\mu$ s 
Applied Pulsed Power, Inc.

Appendix $\mathrm{C}$

Patent Application 
AWP-2

161

INDUCTIVELY GENERATED STREAMING PLASMA ION SOURCE

\section{REFERENCE TO PROVISIONAL APPLICATION}

This application claims an invention which was disclosed in Provisional Application Number 60/515,050, filed 10/28/2003, entitled "INDUCTIVELY GENERATED STREAMING PLASMA ION SOURCE ". The benefit under 35 USC §119(e) of the United States provisional application is hereby claimed, and the aforementioned application is hereby incorporated herein by reference.

\section{ACKNOWLEDGMENT OF GOVERNMENT SUPPORT}

This invention was made with Government support under Grant No. DE-FG02-01ER83147, awarded by the Department of Energy. The government has certain rights in the invention.

\section{FIELD OF THE INVENTION}

15 The invention pertains to the field of ion beam sources. More particularly, the invention pertains to sources for pulsed neutralized ion beams which are used in areas such as material modifications, fusion energy research, and space propulsion.

\section{BACKGROUND OF THE INVENTION}

20 A neutralized ion beam is a stream of ions with directed velocity, accompanied by an equal charge density of electrons that produces a type of net charge-neutral streaming plasma. Such neutral plasma streams allow much higher ion current density to be transported than can be transported with space-charge dominated bare ion beams. There are many different methods 
AWP-2

162

used to create neutralized ion beams. There are continuous generation methods, creating steady streams of ions, and pulsed methods, creating small bursts of ions. These sources have two basic mechanisms. The first is the creation of the ions from a non-ionic source. The second is the acceleration of the ions in a directed beam. The methods for the creation of ions can be from electric field or thermal effects. Either methods results in the separation of one or more electrons from atomic or molecular elements, thus creating an ionized state. The acceleration of the ions can be caused by application of electrostatic or time dependant magnetic fields.

One class of ion sources uses a voltage potential applied between two electrodes to generate

10 an electric field. Free electrons are accelerated by the electric field and collide with gas molecules resulting in a partially ionized gas. Often these sources use thermal effects, such as a hot filament, to increase the number of free electrons available for the ionization process. The electric field across the partially ionized gas may add a directed velocity component to the thermal velocity of the ions, while a magnetic field may be used to focus the ion flow into

15 a beam. Some of these sources are able to run continuously at low currents, others operate pulsed at higher currents. Sources with some of these basic characteristics are described in U.S. Pat. Nos. 6,734,434, 6,724,160, 6,717,155 and 6,696,793 respectively. For many high current applications, sources which use electrodes may not be suitable. That is because electrodes have limited lifetime, may require cooling, and introduce impurities into the

20 plasma. On the other hand, a hot filament requires a separate power supply and also has limited lifetime.

Another type of source uses a high frequency RF transmitter with the antenna immersed in the neutral gas. The electric field component of the transmitted electromagnetic wave is used to break down the gas into plasma. This type of ion source is disclosed in U.S. Pat. No.

$256,664,548$. Typically, the percentage of ionization increases gradually over many cycles before the desired level is obtained. This type of source is most suitable for steady state applications. 
AWP-2

163

Another type of source is inductively driven using one or more coils located near the neutral gas. The coils are driven by one or more half cycles of oscillating current. The induced electric field produced by the changing magnetic field is used to break down the gas while the magnetic field can be used to accelerate the resulting plasma. If a localized pulsed gas source is used, the ions can be accelerated away from the neutral gas, resulting in fully ionized plasma moving with a well defined leading edge. An example of a pulsed ion beam source is described in U.S. Pat. No. 5,525,805, with additional material covered in U.S. Pat. Nos. $5,656,819$ and 5,532,495. Inductively driven sources eliminate the need for electrodes. For rapid ionization of gasses at low pressure with purely inductively driven sources, high rates of

10 change in the driving current are required to produce sufficient inductive electric field for rapid, complete ionization.

All of the above examples use gas as the supply of the molecules to be broken down into ions. Other possibilities are sublimed molecules from heated solids and liquids. In all cases, the supply can be generated in a pulsed or steady flow method. The supply can be near or in the 15 breakdown region or maintain a constant background pressure of the neutral molecules in the breakdown region.

One method for generating a pulsed gas supply is to use a fast gas valve. An example, which describes a fast gas valve operated by pulsing electromagnetic coils to move a magnetic metal disk, is described in U.S. Pat. No. 4,583,710. Another valve, a fast gas valve which uses

20 induced magnetic fields to move a non-magnetic metal disk, is described in U.S. Pat. No. $5,525,805$. This type of fast valve opens and closes faster than the electromagnetic valves, but some of them have problems which reduce the quality and lifetime of the valve. In those valves, a conical metal disk was held against a seal providing an annular puff of gas. However, due to material property differences, clamping forces, adhesion forces, and other

25 factors, one could not be assured of a uniform gas density around the annular puff. Also, deflection of the metal disk results in metal fatigue which can limit the lifetime of the disk.

A neutral beam intensity controller is described in U.S. Pat. No.4,596,687. Neutral beams are beams of neutral atoms, not the net-neutral ionized plasma of equal populations of free 
AWP-2

164

electrons and ions described in this invention, which is described in detail infra. Neutral beams are produced by charge-exchange of an un-neutralized ion beam passing through a neutral gas cell. U.S. Pat. No. 4,596,687 describes a method of controlling the current in a neutral beam by magnetic deflection of the ion beam as it enters the neutralizing gas cell. An amplitude-modulated, rotating magnetic field is applied to deflect the ions in a controlled manner to achieve the desired intensity control of the neutral beam along the beam axis at constant beam energy. The magnetic field deflects the orbits of the individual ions in the gas neutralizer before they charge-exchange to become neutral atoms, so that the peak intensity of the neutral beam is deflected away from an exit aperture downstream of the gas cell. As the

10 magnetic deflection is increased, more of the neutrals miss the exit aperture, and the neutral beam intensity passing out through the aperture is reduced.

Therefore, it is desirous to have an improved plasma ion source in which the prior art systems' shortcomings are overcome.

\section{SUMMARY OF THE INVENTION}

The present invention provides a pulsed ion beam source which uses inductive electric fields for ionizing a low pressure gas and time-varying magnetic fields for accelerating the resultant plasma into a high current, low emittance neutralized ion beam with a velocity along a desired direction and a controllable pulse shape. The source includes a set of coils for generating the 20 electric and magnetic fields.

The present invention provides a pre-ionization method which decreases the required electric field for prompt ionization of the neutral gas thereby increasing the efficiency of the ion source. This pre-ionization method includes a pre-ionization coil which is inductively coupled to the set of coils. The pre-ionization coil possesses a gap, for generating seed 25 electrons, coupled in series with a high impedance circuit element, for limiting the current through the gap. 
AWP-2

165

The present invention provides a modulator for shaping of the neutralized ion beam pulse, the modulator including a set of coils having electric current flowing therein.

The present invention provides a nozzle for shaping the neutral gas pulse, the nozzle disposed at the proximity of the set of coils to create a reservoir for the gas subject to ionization.

5 The present invention provides a tank circuit for efficiently transferring the energy from the power supply to the set of coils. The tank circuit includes the set of coils in parallel with a capacitance.

The present invention provides a fast needle valve, which uses a current through a coil generating a pulsed magnetic field that does not fully penetrate a metallic plate connected to

10 the needle thereby opening the valve.

Accordingly, a streaming plasma ion source with a pre-ionization coil is provided. The source includes: a power supply; a gas supply positioned upstream of the source; a set of coils receiving power from the power supply for conveying an energy for ionization of a gas and acceleration of the resultant plasma, and being insulated from the plasma; and a pre-ionization

15 coil, a wire coil with a gap in series with a high impedance circuit element and inductively coupled thereto, for receiving the energy for ionization.

Accordingly, a streaming plasma ion source with a modulator is provided. The source includes: a power supply; a gas supply position upstream of the source; a set of coils receiving power from the power supply for conveying an energy for ionization of a gas and acceleration 20 of the resultant plasma, and being insulated from the plasma; and a modulator for shaping of the resultant neutralized ion beam pulse, the modulator including a set of coils having electric current flowing therein.

Accordingly, a streaming plasma ion source with a nozzle is provided. The source includes: a power supply; a gas supply position upstream of the source; a set of coils receiving power

25 from the power supply for conveying an energy for ionization of a gas and acceleration of the 
AWP-2

166

resultant plasma, and being insulated from the plasma; and a nozzle disposed at the proximity of the set of coils to create a reservoir for the gas subject to ionization.

Accordingly, a streaming plasma ion source with a tank circuit is provided. The source includes: a power supply; a gas supply position upstream of the source; a set of coils receiving power from the power supply for conveying an energy for ionization of a gas and accelerating the resultant plasma, and being insulated from the plasma; and a tank circuit comprising a capacitance positioned in parallel with the inductance of the set of coils for receiving a supply of energy and outputting the same for ionization and acceleration.

Accordingly, a streaming plasma ion source with a fast needle valve is provided. The source

10 includes: a power supply; a gas supply position upstream of the source wherein the gas supply comprises a fast needle valve, which uses a current through a coil generating a pulsed magnetic field that does not fully penetrate a metallic plate connected to the needle thereby opening the valve; and a set of coils receiving power from the power supply for conveying an energy for ionization of a gas and accelerating the resultant plasma, and being insulated from 15 the plasma.

A streaming plasma ion source is provided. The source includes: a power supply; a gas supply position upstream of the source wherein the gas supply comprises a fast needle valve, which uses a current through a coil generating a pulsed magnetic field that does not fully penetrate a metallic plate connected to the needle thereby opening the valve; a set of coils 20 receiving power from the power supply for conveying an energy for ionization of a gas and acceleration of the resultant plasma, and being insulated from the plasma; a modulator for shaping of the resultant neutralized ion beam pulse, the modulator including a set of coils having electric current flowing therein; a nozzle disposed at the proximity of the set of coils to create a reservoir for the gas subject to ionization; a tank circuit comprising a capacitance in

25 parallel with the inductance of the set of coils for receiving a supply of energy and outputting the same for ionization and acceleration; and a pre-ionization coil being a wire coil with a gap positioned in series with a high impedance circuit element and inductively coupled thereto, for receiving the energy for ionization. 
AWP-2

\section{BRIEF DESCRIPTION OF THE DRAWING}

FIG. 1 shows a section cut side view of the plasma ion source.

FIG. 2 shows a front view of the modulator plate.

$5 \quad$ FIG. 3 shows a section cut side view of the axial gas puff valve.

FIG. 4 shows a back view of the axial gas puff valve.

FIG. 5 shows the current through the driver coils.

FIG. 6 shows the current through the modulator coils.

FIG. 7 shows a simplified circuit schematic for the plasma ion source.

10 FIG. 8 shows a set of coils inductively coupled to a separate coil.

\section{DESCRIPTION OF THE PREFERRED EMBODIMENT}

This section includes the descriptions of the present invention including the preferred embodiment of the present invention for the understanding of the same. It is noted that the

15 embodiments are merely describing the invention. The claims section of the present invention defines the boundaries of the property right conferred by law.

Referring to Figs 1-8, specifically referring now to Fig. 1 wherein the basic structure of the inductively generated streaming plasma ion source of the present invention is depicted. The source comprises a backplane 1, a nozzle 2, and a modulator 6 . The backplane 1 includes a set

20 of coils $3,4,5$, mounting holes 10 , and a place or region to insert a gas supply 9 . The nozzle 2 is shaped like a cup with a set of mounting holes 11,12 or outer mounting holes 11 and central mounting holes 12 on the bottom or a first side of nozzle 2 . The outer mounting holes 11 are used to mount the nozzle 2 onto a member. The central mounting hole 12 is used to 
AWP-2

168

mount additional components inside the nozzle 2 as needed. For example, one such component is a gas diverter 13 , which is used to redirect gas from an axial puff into an annular puff originating from gas supply 9.

The modulator 6 also contains a set of coils 7, 8. Also refer to FIG. 2, the mounting holes 16 and a more detailed depiction of the modulator coil 7, 8, locations on the modulator 6 are shown.

Gas released from the gas supply 9 flows out into the nozzle 2, where the gas flows in all directions at the same rate. Gas not flowing out radially along the backplane 1 will be contained by the reservoir 14 created by the nozzle 2 in which reservoir 14 is enclosed. The

10 present invention teaches the controlling of the spacing between the nozzle 2 and the backplane 1, as well as the inner and outer diameters and lengths of the nozzle 2 . The effect is that the present invention teaches the controlling of the profile of the gas density versus time at locations in front of the backplane 1, outside the radius of the nozzle 2. In other words, the present invention controls the profile of the gas density versus time at region 15 outside

15 nozzle 2. The gas subject to ionization exits the nozzle 2, and then travels past the coils 3 , 4, 5 , on the backplane 1 .

The inner most coil 5, is used as a pre-ionization coil. The coil 5 is a wire with a gap 102. In series with the gap 102 is a capacitor 90, which limits the current able to flow within the wire. A first voltage is induced across the gap 102 by the oscillating magnetic field created by the 20 driving coils 3, 4. This first voltage across gap 102 creates seed electrons, which aid in the breakdown of neutral gas into plasma. Controlling the capacitance 90 and the width of the gap 102 as well as the diameter of the coil 5, controls the amount of seed electrons generated while limiting the current and thereby eliminating lifetime concerns for the electrodes 104 at the edges of the gap 102. It is known that the amount of electrons in neutral gas at room

25 temperature is insufficient for initiating rapid ionization. The present invention provides more free electrons allowing rapid ionization. 
AWP-2

169

The seed electrons travel out into the neutral gas in front of the driving coils 3,4 , on the backplane 1 . While only two coils 3, 4 are shown, any number of coils such as coil 3, 4 may be used. These coils may be connected in series. A capacitance 73 may be coupled in series with the coils. Wires in the driving coils 3, 4 are connected in series with the current 60 , shown in FIG. 5, traveling in the same direction in all driving coils 3, 4. This oscillating current 60 generates oscillating electric and magnetic fields. The circuit voltage 61 , also shown in FIG. 6, is the voltage across the capacitance 73. In addition, voltage 61 is proportional to the rate of change of the current 60 , multiplied by the inductance of the tank circuit 71. The magnetic field is proportional to the current 60 , in the driving coils 3 , 4. This 10 oscillating magnetic field induces voltage 61 across the gap 102 in the pre-ionization coil, 5. The voltage in the space between the driving coils 3,4 , is equal to the total electric potential 61 , across all the coils 3, 4, divided by the number of coils. This electric field working on free electrons, initiated by the seed electrons generated by the pre-ionization coil 5 , breaks down the neutral gas into plasma by ionizing collisions between the accelerated electrons and the

15 neutral gas. The charged particles, ions and electrons, comprising the plasma are then accelerated by the oscillating magnetic field.

The acceleration drives the plasma away from the driving coils 3, 4. The acceleration occurs because the plasma conductivity becomes high, thereby allowing a plasma current to be induced by the oscillating magnetic field. This induced plasma current in the applied, 20 oscillating magnetic field generates the repulsive force which accelerates the plasma away from the driving coils 3,4 .

The streaming plasma moves downstream past the nozzle 2, towards the modulator 6 . As the plasma approaches the modulator, current 62 from a current source, shown in FIG. 6, is driven into the wires in the coils 7, 8 of the modulator 6 . This current again generates electric and 25 magnetic fields. These fields act on the plasma like a valve, restricting or preventing flow of plasma through the gaps 17 in the modulator 6. By controlling the timing and shape of the current of the coils 7,8 , modulator 6 controls the profile of the ion current passing through the modulator 6. 
AWP-2

170

Referring specifically to Fig. 3, an example of a gas supply using the axial puff valve is shown. The body of the valve includes a two piece body, a rear section 29 and a forward section 31 . The valve is a fast needle valve using a stem 40 , which is sealed against a plate 34 for controlling the gas flow. The plate 34 is sealed by an o-ring 35 to the forward body section 31 by affixing means such as screws 39. The plate 34 can be made of plastic or polymer materials such as Teflon or metal, provided a sufficient seal can be obtained between the end of the stem, 40 and the plate 34 . An alternative method would be to make the plate 34 out of two pieces comprising a first piece comprising layer of plastic and a second piece comprising layer of metal. The seal is then disposed between the stem 40 and the plastic layer

10 with the metal layer providing structural strength in order to prevent the sealing force from deforming the plastic. The stem 40 is attached to a non-magnetic, highly electrically conductive, metal disk 30 by attaching means such as a nut 27 . The axial motion of the stem 40 is controlled by bearings 28,36 , located in the forward body section, 31 . A spring 23 between the metal disk 30 and the rear body section 29 forces the stem 40 against the plate 34 .

15 The control coil 33 is held by an inner coil form 41 and an outer coil form 32 . Both the inner coil form 41 and the outer coil form 32 are disposed inside the forward body section 31, under a metal disk 42. The two coil forms 32, 41, are made of a non-conducting material. The area in the forward body section 31 under a metal cover 42 is sealed by o-rings 22, 25 for separation from the gas supply area 26 disposed in the rear body section 29 . The metal cover

2042 is made from a material penetrable to magnetic field, such as titanium. When a current pulse flows through the control coil 33, the magnetic field generated by the current pushes the metal disk 30 away from the control coil 33, thereby compressing the spring, 23. The current pulse causes the stem 40 to move away from the plate 34 , thereby allowing gas located in a second reservoir 38 to escape through the exit, 37 . The metal disk 30 bounces off the rear 25 body section 29, but quickly returns the stem 40 to the previous position before the current pulse is applied, thereby sealing against the plate 34 as before. The opening time of the valve is controlled by various elements including the distance of the gap 100 between the forward and rear body sections 29, 31 the pulse width and amplitude of the current pulse, the force generated by the spring 23 , and the mass of the stem 40 , metal disk 30 , and nut 27 . An 
AWP-2

171

elastomer 24 over the metal cover 40 prevents the metal disk 30 from bouncing which assures the valve only opens once per current pulse. The reservoir 38 is then refilled by a channel between the stem 40 and the forward body section 31 from the gas supply entrance 26 in the rear body section 29. The valve is designed to be used in pulsed operation. Either the forward o-ring 44 or the rear o-ring 20 using blind tapped holes 21,43 seal the valve in the source.

FIG. 4 shows a recess 51 in the forward body section 31 and an opening 52 in the rear body section 29, which are used to connect the control coil 33 to the control circuit. This opening or hole 52 allows the control coil 33 to be in standard atmosphere rather than having to encapsulate the control coil 33 to allow it to be in vacuum. This feature significantly eases the assembly and manufacturing of the valve. Also shown are a set of holes 50 for attaching the front body section 31 to the rear body section 29 .

A simplified electrical schematic of the driver circuit is shown in FIG. 7. The driver circuit includes a pulsed power circuit 76 connected via a coaxial cable 79 to a tank circuit 77 . The pulsed power circuit 76 comprises a pulsed power supply 75, which can be a capacitor

15 connected to a high voltage DC power supply, a switch, 78, which may be a solid state switch, and diodes 74. The driver circuit has a single point electrical ground connection 80 in the pulsed power circuit 76 . The tank circuit 77 includes a tank capacitor 73, and the driver coils 3 , 4. The driver current 60 is measured at the return current path 70 using a Rogowski. The induced electric potential 61 across the driver coils 3, 4 is measured across the tank circuit 71,

2072 . The diodes 74 and the inductance of the coaxial cable 79 prevent the energy injected into the tank circuit 77 from returning back to the pulsed power supply 75 .

Referring specifically to Fig. 8, a schematic depiction of the coils 3, 4, 5 is shown. Coils 3, 4, 5 may be positioned on a plane such as backplane 1. Coil 3 and coil 4 are electrically coupled in series and insulated by suitable insulation materials. Capacitance 73 (see Fig. 7) is coupled to the coils 3, 4 for oscillatively discharging inputted energy stored therein. Coil 5 may be disposed concentrically within coils 3, 4 and inductively coupled therewith for inductively receiving energy discharged from capacitance 73 . In turn, a complete electric circuit is formed by coil 5, capacitance 90, electrodes 104, and gap 102. Capacitance 90 uses the 
AWP-2

172

inductively received energy and oscillatively discharging the same via coil 5 and gap 102 . backplane further has mounting holes 93 circumferentially distributed thereon. Coils 3, 4 have leads 91, 92 respectively for coupling the capacitance 73 .

A neutralized ion beam source is described in this invention which uses pulsed inductive electric fields to ionize a low pressure gas. The techniques of the present invention includes a pre-ionization scheme or method, wherein an axial valve of Figs. 3-4, a nozzle 2, a tank circuit 77, and a modulator 6 are provided to improve the efficiency of the ionization and to produce a pulsed, high current, low emittance, neutralized ion beam that has a directed velocity, a controllable pulse shape and is fully ionized downstream from the source.

10 The ion source uses a high speed gas valve to inject a puff of gas through a nozzle 2 and into an annular volume 15 between two concentric driving coils. The driving coils are driven with an oscillating current pulse. The high rate of change in the current driven through the driving coils 3, 4 produces a rapidly rising magnetic field in the gas volume, inducing an azimuthal electric field in the gas in the order of, for example $100 \mathrm{~V} / \mathrm{cm}$. While the electric field initiates

15 and drives a rapid breakdown of the gas into plasma, the rapidly changing magnetic field, produced by the current in the driving coils 3,4 , induces currents in the plasma and thereby accelerates the resulting plasma. Downstream from the driving coils 3 , 4, a modulator 6 , a pair of concentric coils 7, 8 placed so that the ion beam passes between them is used to control the pulse shape of the ion beam current. In an alternative embodiment, the gas valve 20 may not be required; a static gas fill or flowing gas may be used.

The pulsed ion source of the present invention has inherent advantages compared with prior art sources, which use electrodes to generate the electric fields required to break down neutral gas into plasma or accelerate the ions. That is because electrodes have limited lifetime, may require cooling, and introduce impurities into the plasma. These impurities typically are strongly radiating species which dissipate energy, cool the plasma thereby preventing full ionization, and reduce the energy efficiency of the plasma source. These problems are eliminated by the present invention because the driving coils 3, 4 are electrically insulated 
AWP-2

173

from the plasma as there is no plasma current that connects to the driving coils 3,4 .

Therefore, no impurities can originate from the insulated coils 3, 4 .

The ionization process is initiated when seed electrons, accelerated by the inductive electric field, have ionizing collisions with a neutral gas. By way of example, an electric field, of the order of $100 \mathrm{~V} / \mathrm{cm}$ is required for prompt initiation and completion of the ionization process. Since the ionization coil must have current driven in it to produce the changing magnetic flux that generates the inductive electric field, the higher that electric field must be or the longer it must be applied results in more energy that must be expended in the electric driving circuit. The high voltage on the ionization coil leads to high peak current which may far exceed the current needed in the discharge to drive the plasma to full ionization.

The seed electrons, needed for reproducible and prompt gas breakdown, can be created using a localized high magnitude electric field or thermal effects. Localized high magnitude electric fields can be generated from sharp edges of metal members, at a first electric potential, near a second electric potential. The source of the present invention uses a new device called a pre-

15 ionization coil 5 . This pre-ionization coil 5 is inductively coupled to the driving coils. The pre-ionization coil 5 is typically concentric with the coils but at a smaller radius. By placing a small spark gap in the pre-ionization coil 5, an electric field, with a magnitude many times that of the azimuthal field, is produced in the gap. This electric field generates seed electrons. To reduce total current through the gap, which could otherwise cause wear to the gap

20 electrodes and emit impurities into the plasma as described earlier with regarding to related prior art methods and apparatus, a high impedance circuit element, such as a low capacitance capacitor 73, is connected in series with the gap. By placing the pre-ionization gap near the circumference of the driving coils, the seed electrons are emitted directly into the source gas in the breakdown region. This method reduces the inductive electric field required for prompt 25 ionization of the neutral gas into plasma, and improves reproducibility of the breakdown.

To create the required electric and magnetic fields to break down a neutral gas and accelerate the resultant plasma, a large oscillating current is required. The energy required is proportional to the current squared times the inductance. If the power supply for this current, 
AWP-2

174

which can be a capacitor charged to the appropriate voltage in series with a switch, directly drives the ionization coils then this inductance is the sum of the inductance of the ionization coil, power feed and driver. In the present invention, in order to minimize the drive energy, low inductance switches and capacitors are necessary and the driver is connected to the coil using a short, low inductance feed.

To decrease the energy required and remove some of the constraints on the driver, the source uses a capacitor across the driving coils as a tank circuit. While tank circuits are known, the specifications and use of the circuit is novel. Note that the driving coils 3, 4 are part of the tank circuit 77 in that they provide the requisite inductance of the circuit 77 . The tank circuit

1077 is charged with one or more half sine waves from an external pulsed power supply. The capacitance of the tank circuit 77 needs to be harmonically tuned with the inductance of the driving coils 3, 4 to generate a pulse shape of a specific form. The current 60 through the driving coils 3, 4 then has the form of a large amplitude first half sine wave with a long period followed by smaller amplitude oscillations with a shorter period. The large initial amplitude

15 provides the required electric fields necessary to break down the neutral gas into plasma while the higher frequency oscillations create the required magnetic field pulses that accelerate the ions. The energy for these oscillations is provided by the tank circuit. The tank capacitor 73 can be placed close to the coils so that the ratio of the ionization coil inductance to the total circuit inductance is near one for high efficiency. Alternatively, the pulsed power supply can 20 have a higher inductance and be located at a convenient distance from the source without compromising efficiency.

High neutral gas density can cause degradation to the ion beam as a result of collisions between the steaming ions and neutrals. To prevent this, an ion source has to either operate under conditions which result in small collision cross sections such as using a very low 25 background pressure of the supply gas, or use a localized supply 9 near the breakdown region. Also, the pressure in the breakdown region 15, which is in front of the driving coils, has an effect on the quality of the ion beam. Typical room temperature gas velocities are significantly lower than the directed velocity of the ion beam, so a pulsed or constant flow 
AWP-2

175

localized gas supply 9 located near the breakdown region 15 may be used. For this source, to provide the lowest downstream neutral gas density while still providing the correct neutral gas density in breakdown region 15, a special nozzle 2 was invented in the present invention to control the gas supply flow. The nozzle 2 uses a gas supply 9 located at the center of the driving coils. The gas is directed radially out from the center. Then the gas enters into a large reservoir in the shape of a cup. There is a small gap between the plane of the driving coils, which is a solid structure, and the end of the cup. The gas exits from the reservoir radially, passing in front of the driving coils. Using this nozzle 2 design, the gas flow moves perpendicular to the ion beam.

10 A constant flow gas supply may require more vacuum pumping than a pulsed gas supply to maintain low enough pressure for good propagation of the beam. Further, since the preferred embodiment uses a pulsed source, a constant gas supply is not required for the same. Another advantage for using a pulsed source is that the operating conditions of the gas density in front of the driving coils 3, 4 can be controlled by changing the timing from the opening of the

15 valve to the start of the current pulse 60 in the driving coils 3,4 .

For the ion source of the present invention, a new modified fast needle valve (see Fig. 3) was invented to provide a uniform puff of gas. A pulsed magnetic field operates the valve. By way of an example, a half sine wave current pulse, of a peak of hundreds to thousands of amperes, is sent through a coil 33 above a metal disk 30 or plate. The current pulse width, and the

20 conductivity and thickness of the disk 30, are selected such that the magnetic field produced by the coil 33 does not fully penetrate the disk 30 during the current pulse. As a result, the pulsed magnetic field accelerates the metal disk 30 away from the coil 33 . Attached to the metal disk 30 is a needle. The needle is therefore accelerated away from its seat, thereby opening the valve. After the magnetic field pulse, the needle is pushed back by spring force, 25 either from a spring 23 located under the disk 30 or by shaping the disk 30 conically in the form of a Belleville spring. The spring force keeps the valve sealed until the next magnetic field pulse. The mass of the needle and plate can be made sufficiently small, resulting in a large acceleration. If the current pulse is only tens of microseconds long and the metal disk 30 
AWP-2

176

is only allowed thousandths of inches of motion, than the valve is only open for tens of microseconds. Behind the seat is a small plenum which is connected to a gas source. During the time the valve is open, most of volume of gas in the plenum escapes from the valve. Between the pulses, the plenum is refilled with gas. The size of the gas plenum and the pressure of the gas in the plenum can be adjusted to control the volume of gas released. Combined with the previously described nozzle, an annular gas puff with very good azimuthal uniformity can be produced. Obtaining good azimuthal uniformity has been a problem with prior art fast gas valves. To further improve the operation of the valve, an elastomer 24 may be used to absorb the kinetic energy in the metal disk 30 and prevent it from bouncing as it is

10 closing. In other words, the impact of the metal disk 30 upon a third hard material member is impeded by the use of the elastomer 24 .

The source as described to this point produces a pulse of streaming plasma with each pulse of the gas valve and driver coils. The ion current density transported by this streaming plasma pulse rises smoothly to a peak and then declines back to zero over the time of the pulse. The

15 time duration of this pulse can be controlled by the characteristics of the driving coil waveform, but the output is always peaked in due time. For some applications, further control of the ion current density pulse shape may be required. In particular, for ion sources for particle accelerators, it is desired to produce a pulse with fast rise- and fall-time, and a very constant current density in between, i.e., a flat pulse. The present invention teaches a

20 modulator for modulating the resultant ion current in that further shaping of the pulse is possible. In other words, the modulator provides further shaping of the pulse beyond what is obtainable with the driver coils alone.

The modulator operates by placing a pair of concentric modulator coils downstream of the breakdown region, positioned so that the plasma streams through the annular space between 25 these modulator coils. A pulsed current waveform is imposed on the modulator coils, coinciding with the time during the passage of plasma through the plane of the coils. As the streaming plasma encounters the magnetic field produced by the modulator current, a current is induced in the conductive plasma, producing a magnetic force on the plasma in the 
AWP-2

177

direction which would repel the plasma axially away from the modulator, back toward the source. A large enough current in the modulator coils can essentially stop the plasma, but smaller current can attenuate the amount of plasma that passes through the modulator to exit downstream. The degree of attenuation is determined by the magnitude of the current in the modulator coils. Therefore, by choosing an optimal pulse shape for the magnitude of the modulator coil current, the attenuation of the plasma pulse can be controlled as a function of time throughout the pulse, thus controlling the shape of the plasma pulse transmitted to the exit.

The fundamental feature of this interaction of the neutralized ion beam, or plasma stream,

10 with the modulator is that the plasma density and conductivity are high enough so that the plasma acts as a conductive, net charge-neutral medium. This is in distinction to the case of a bare, un-neutralized ion beam, which would interact very differently with the magnetic field of the modulator. In the case of the latter, each ion, as it passed through the modulator field, would simply be deflected at an angle to its original direction of flight. This does not happen

15 with the streaming plasma source of the present invention because the co-moving electrons in the plasma are deflected in the opposite direction, producing charge separation and thereby generating an electrostatic field in the radial direction that tends to cancel the magnetic deflection of ions. This is known as polarization-drift propagation. Thus, by way of experiment, no net deflection of the beam exiting the modulator is observed in this source, 20 only the current density is attenuated by an amount dependent on the modulator field strength. With an un-neutralized ion beam, a deflection proportional to the field strength would be produced. It is noted that the streaming plasma source avoids this deflection, which would have an unacceptable adverse affect on the ion optics of the source for critical applications such as high-energy ion accelerators.

In contrast to this prior art, the ion source described in this invention uses a magnetic field to control the ion current density in the plasma stream produced by the source, but this field operates on the plasma as a conductive medium, rather than as individual ions, and 
AWP-2

178

produces control of the ion current density without deflection, which is required for some applications.

The application of the present invention may comprise fusion energy related industries, semiconductor industries, nanotechnology related fields, material modification, such as

5 hardening and smoothing, related industries, space propulsion related industries, etc.

Accordingly, it is to be understood that the embodiments of the invention herein described are merely illustrative of the application of the principles of the invention. Reference herein to details of the illustrated embodiments are not intended to limit the scope of the claims, which themselves recite those features regarded as essential to the invention. 
AWP-2

179

Fig. 1

$1 / 8$
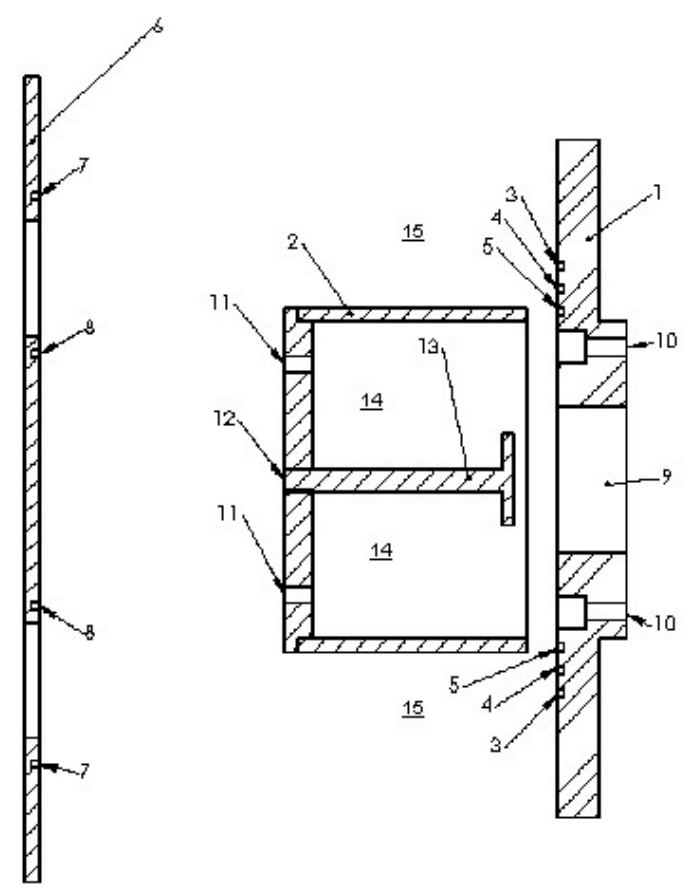
AWP-2

180

Fig. 2

28

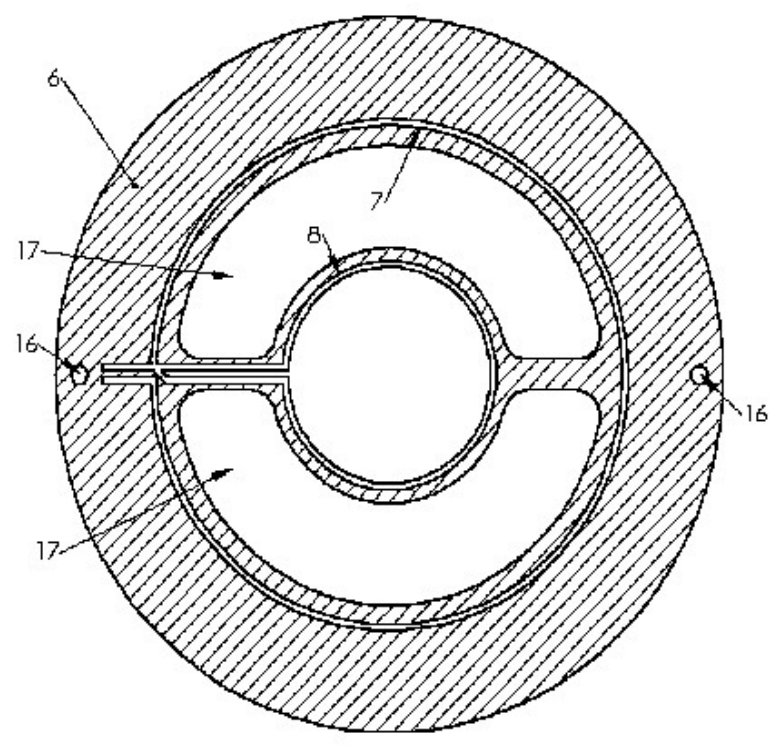


AWP-2

181

Fig. 3

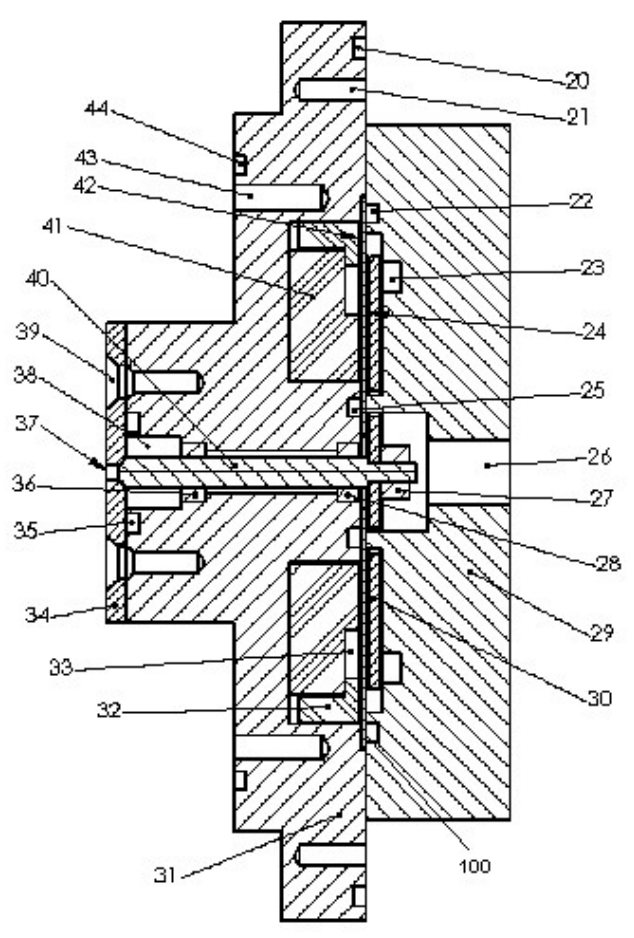


AWP-2

182

Fig. 4

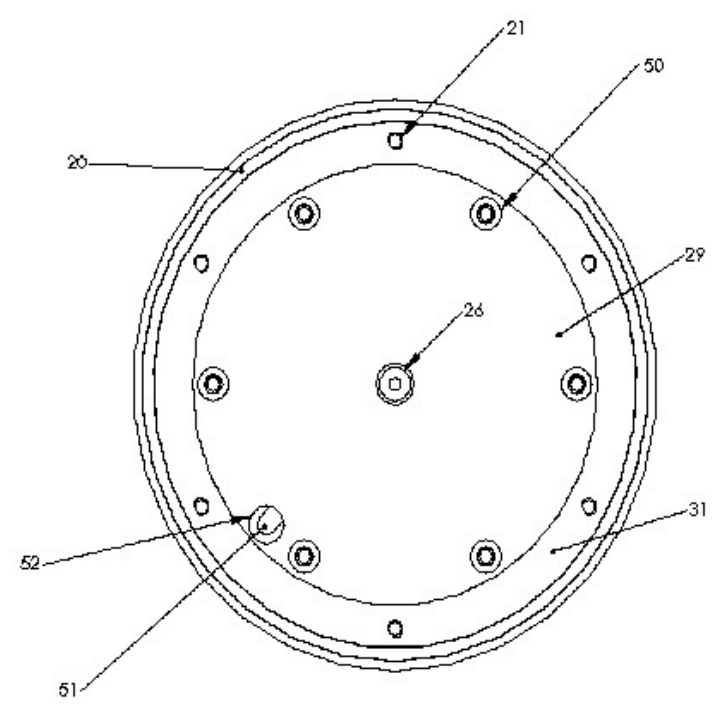


AWP-2

Fig. 5

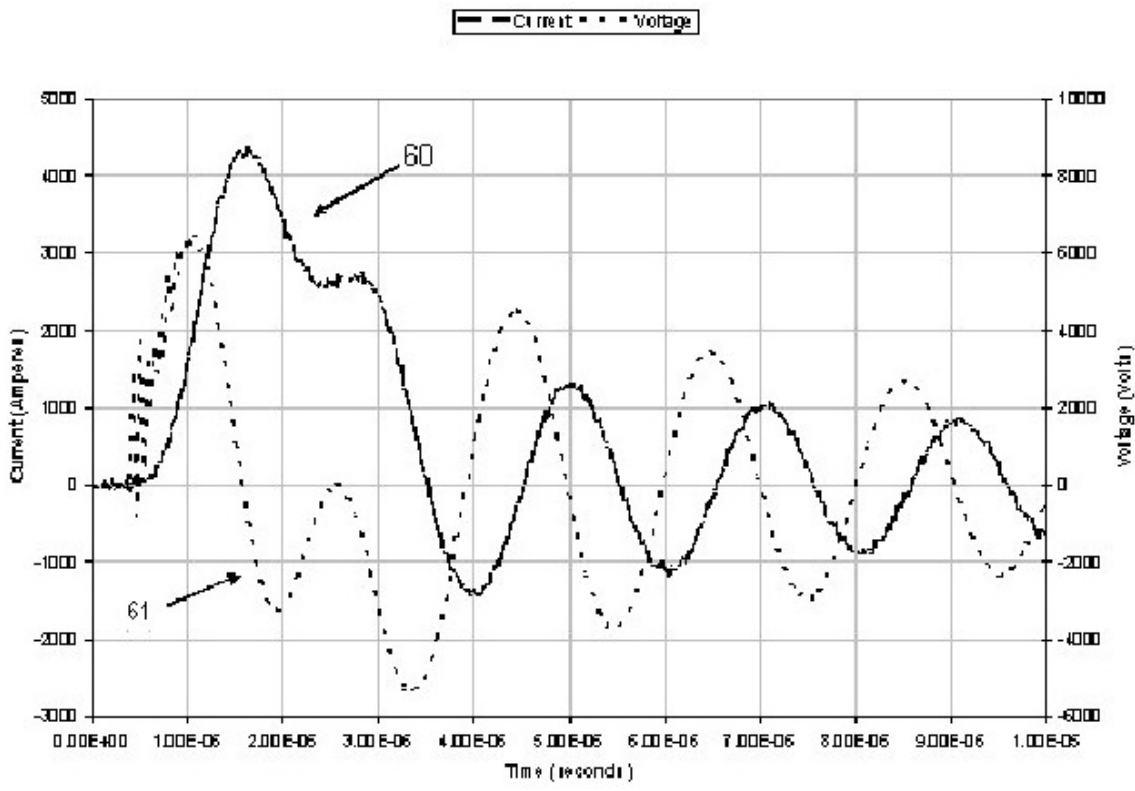


AWP-2

Fig. 6

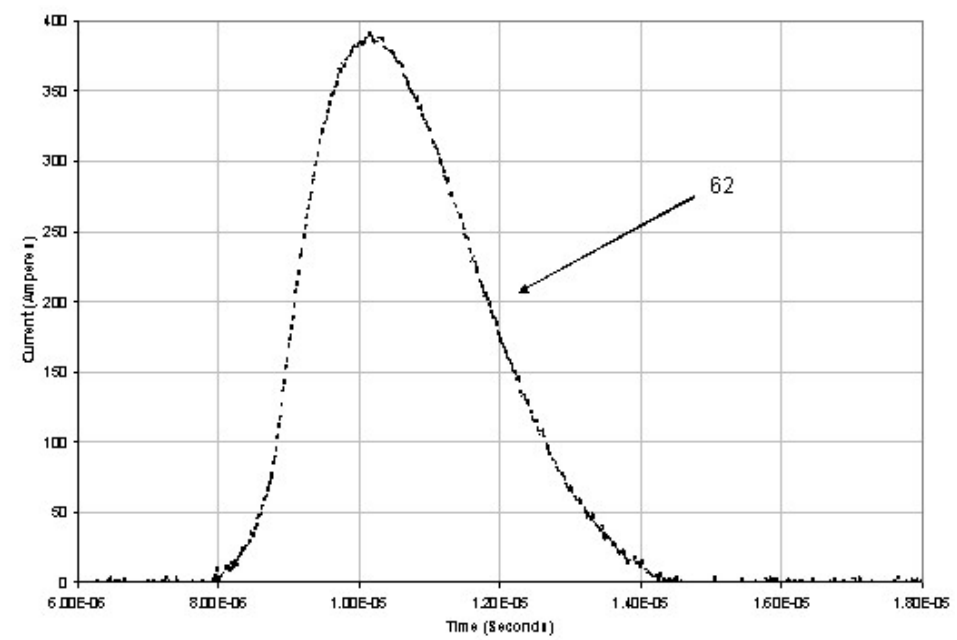

Fig. 7

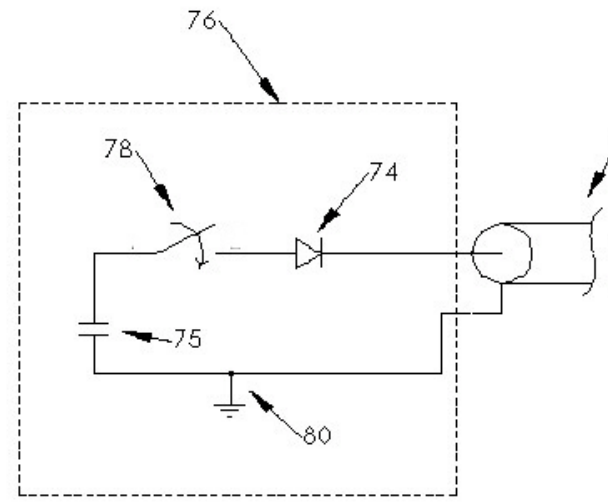




\section{AWP-2}

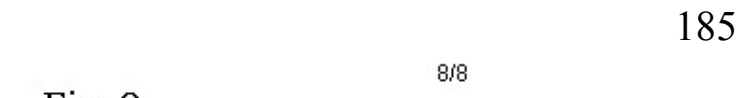

Fig. 8

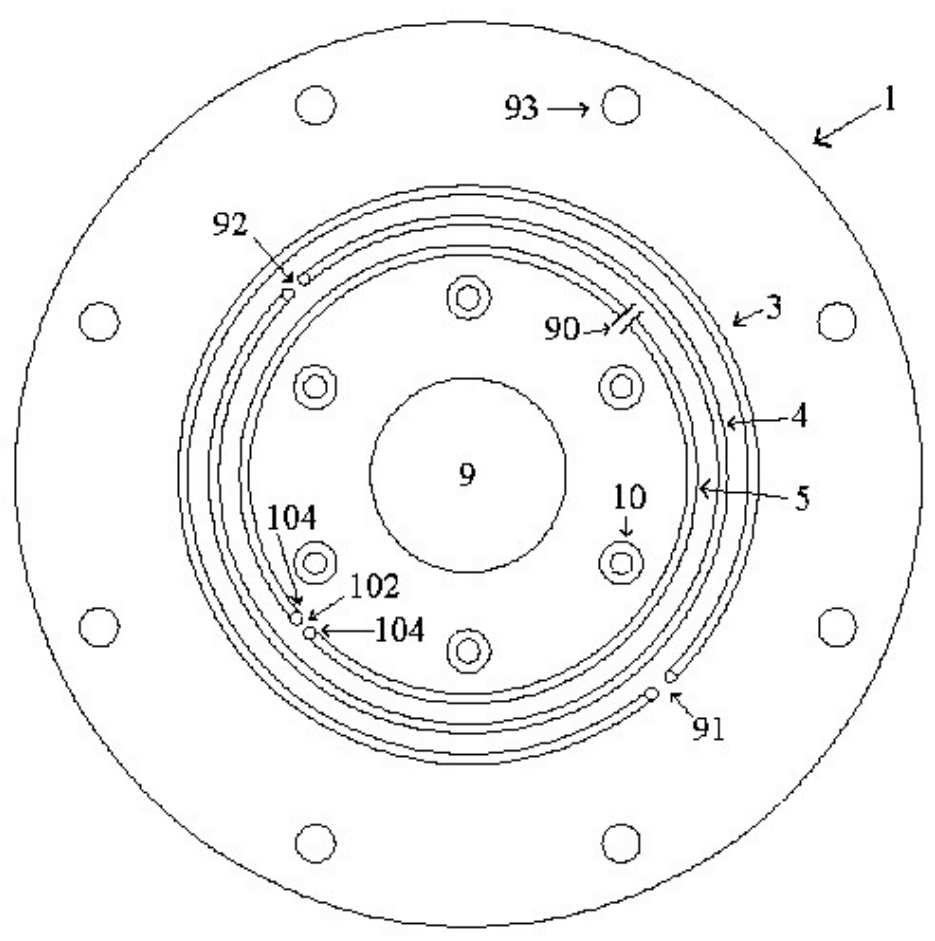


AWP-2

186

ABSTRACT

A novel pulsed, neutralized ion beam source is provided. The source uses pulsed inductive breakdown of neutral gas, and magnetic acceleration and control of the resulting plasma, to form a beam. The beam supplies ions for applications requiring excellent control of ion species, low remittance, high current density, and spatial uniformity 